\title{
On the automatic design of decision-tree induction algorithms
}



SERVIÇO DE PÓS-GRADUAÇÃO DO ICMC-USP

Data de Depósito:

Assinatura:

\title{
On the automatic design of decision-tree induction algorithms
}

\author{
Rodrigo Coelho Barros
}

Advisor: Prof. Dr. André C. P. L. F. de Carvalho

Co-Advisor: Prof. Dr. Alex A. Freitas

Doctoral dissertation submitted to the Instituto de Ciências Matemáticas e de Computação - ICMC-USP, in partial fulfillment of the requirements for the degree of the Doctorate Program in Computer Science and Computational Mathematics. FINAL VERSION.

USP - São Carlos

February 2014 
Ficha catalográfica elaborada pela Biblioteca Prof. Achille Bassi e Seção Técnica de Informática, ICMC/USP, com os dados fornecidos pelo(a) autor(a)

Barros, Rodrigo $\mathrm{C}$.

B2770 On the automatic design of decision-tree induction algorithms / Rodrigo C. Barros; orientador André C. P. L. F. de Carvalho; coorientador Alex A. Freitas. -- São Carlos, 2014. 204 p.

Tese (Doutorado - Programa de Pós-Graduação em Ciências de Computação e Matemática Computacional) -Instituto de Ciências Matemáticas e de Computação, Universidade de São Paulo, 2014.

1. decision trees. 2. hyper-heuristics. 3 . evolutionary algorithms. 4. automatic design. I. de Carvalho, André C. P. L. F., orient. II. Freitas, Alex A., co-orient. III. Título. 
Sobre o projeto automático de algoritmos de indução de árvores de decisão

\author{
Rodrigo Coelho Barros
}



SERVIÇO DE PÓS-GRADUAÇÃO DO ICMC-USP

Data de Depósito:

Assinatura:

\title{
Sobre o projeto automático de algoritmos de indução de árvores de decisão
}

\author{
Rodrigo Coelho Barros
}

\author{
Orientador: Prof. Dr. André C. P. L. F. de Carvalho \\ Co-orientador: Prof. Dr. Alex A. Freitas
}

\begin{abstract}
Tese apresentada ao Instituto de Ciências Matemáticas e de Computação - ICMC-USP, como parte dos requisitos para obtenção do título de Doutor em Ciências - Ciências de Computação e Matemática Computacional. VERSÃO REVISADA
\end{abstract}


Ficha catalográfica elaborada pela Biblioteca Prof. Achille Bassi e Seção Técnica de Informática, ICMC/USP, com os dados fornecidos pelo(a) autor(a)

Barros, Rodrigo $\mathrm{C}$.

B2770 On the automatic design of decision-tree induction algorithms / Rodrigo C. Barros; orientador André C. P. L. F. de Carvalho; coorientador Alex A. Freitas. -- São Carlos, 2014. 204 p.

Tese (Doutorado - Programa de Pós-Graduação em Ciências de Computação e Matemática Computacional) -Instituto de Ciências Matemáticas e de Computação, Universidade de São Paulo, 2014.

1. decision trees. 2. hyper-heuristics. 3 . evolutionary algorithms. 4. automatic design. I. de Carvalho, André C. P. L. F., orient. II. Freitas, Alex A., co-orient. III. Título. 
"There is grandeur in this view of life, with its several powers, having been originally breathed into a few forms or into one; and that, whilst this planet has gone cycling on according to the fixed law of gravity, from so simple a beginning endless forms most beautiful and most wonderful have been, and are being, evolved."

Charles Robert Darwin On the Origin of Species 

Aos meus pais, Marta e Luís Fernando Barros, que me apoiaram quando decidi deixar o Rio Grande do Sul e iniciar esta jornada. Deles nunca me faltaram palavras de conforto ou suporte emocional e financeiro. Não poderia desejar melhores pais.

À minha avó, Lygia Vernetti, a quem dedico esta tese por ser uma das pessoas mais especiais da minha vida. $\mathrm{O}$ fato de eu poder redigir esta tese em inglês se deve em grande parte a ela.

À minha mulher, Alessandra Duarte, a quem também dedico esta tese. Tornou suportáveis os momentos difíceis e adoçou os momentos felizes. Não há no mundo quem me faça mais feliz.

À minha tia, Mônica Coelho, e aos meus amigos, Matheus Braga e Sabrina Rocha, por terem me oferecido estadia e bom papo nesses 4 anos de viagens passando por Porto Alegre.

Ao amigo e parceiro de pesquisa Márcio Basgalupp. Parece que faz um século que fizemos nosso primeiro artigo em parceria, na PUC-RS em 2008, e quem diria que a parceria se estenderia por tanto tempo ou que teria tanto sucesso. Serei sempre grato por teres me apresentado ao mundo da computação evolutiva.

Ao meu orientador, professor André Carvalho, uma das melhores pessoas que já conheci. Profissional exemplar que consegue aliar sucesso profissional invejável à humildade e gentileza. Certamente será minha principal fonte de inspiração na tentativa de ser um bom profissional e uma boa pessoa.

Ao meu co-orientador, Alex Freitas, que foi impecável ao me receber em Canterbury, UK, entre 2012 e 2013. Estendo o meu agradecimento aos alunos dele de doutorado: Suwimol, Khalid e Cen, por um ano agradável na University of Kent.

Ao meu orientador de mestrado, Duncan Ruiz, por ser uma pessoa importante na minha formação profissional.

Aos amigos do GPIN-PUCRS e BIOCOM-USP, com quem dividi grandes momentos de minha vida. Espero nunca perder o contato com vocês.

À FAPESP, pelo suporte financeiro que tornou possível a realização deste trabalho. 

Abstract

$\mathrm{D}$ Ecision-tree induction is one of the most employed methods to extract knowledge from data. There are several distinct strategies for inducing decision trees from data, each one presenting advantages and disadvantages according to its corresponding inductive bias. These strategies have been continuously improved by researchers over the last 40 years. This thesis, following recent breakthroughs in the automatic design of machine learning algorithms, proposes to automatically generate decision-tree induction algorithms. Our proposed approach, namely HEAD-DT, is based on the evolutionary algorithms paradigm, which improves solutions based on metaphors of biological processes. HEAD-DT works over several manually-designed decision-tree components and combines the most suitable components for the task at hand. It can operate according to two different frameworks: i) evolving algorithms tailored to one single data set (specific framework); and ii) evolving algorithms from multiple data sets (general framework). The specific framework aims at generating one decision-tree algorithm per data set, so the resulting algorithm does not need to generalise beyond its target data set. The general framework has a more ambitious goal, which is to generate a single decision-tree algorithm capable of being effectively applied to several data sets. The specific framework is tested over 20 UCI data sets, and results show that HEAD-DT's specific algorithms outperform algorithms like CART and C4.5 with statistical significance. The general framework, in turn, is executed under two different scenarios: i) designing a domain-specific algorithm; and ii) designing a robust domain-free algorithm. The first scenario is tested over 35 microarray gene expression data sets, and results show that HEAD-DT's algorithms consistently outperform C4.5 and CART in different experimental configurations. The second scenario is tested over 67 UCI data sets, and HEAD-DT's algorithms were shown to be competitive with C4.5 and CART. Nevertheless, we show that HEAD-DT is prone to a special case of overfitting when it is executed under the second scenario of the general framework, and we point to possible alternatives for solving this problem. Finally, we perform an extensive experiment for evaluating the best single-objective fitness function for HEAD-DT, combining 5 classification performance measures with three aggregation schemes. We evaluate the 15 fitness functions in 67 UCI data sets, and the best of them are employed to generate algorithms tailored to balanced and imbalanced data. Results show that the automatically-designed algorithms outperform CART and C4.5 with statistical significance, indicating that HEAD-DT is also capable of generating custom algorithms for data with a particular kind of statistical profile. 

Rvores de decisão são amplamente utilizadas como estratégia para extração de conhecimento de dados. Existem muitas estratégias diferentes para indução de árvores de decisão, cada qual com suas vantagens e desvantagens tendo em vista seu bias indutivo. Tais estratégias têm sido continuamente melhoradas por pesquisadores nos últimos 40 anos. Esta tese, em sintonia com recentes descobertas no campo de projeto automático de algoritmos de aprendizado de máquina, propõe a geração automática de algoritmos de indução de árvores de decisão. A abordagem proposta, chamada de HEAD-DT, é baseada no paradigma de algoritmos evolutivos. HEAD-DT evolui componentes de árvores de decisão que foram manualmente codificados e os combina da forma mais adequada ao problema em questão. HEAD-DT funciona conforme dois diferentes frameworks: i) evolução de algoritmos customizados para uma única base de dados (framework específico); e ii) evolução de algoritmos a partir de múltiplas bases (framework geral). O framework específico tem por objetivo gerar um algoritmo por base de dados, de forma que o algoritmo projetado não necessite de poder de generalização que vá além da base alvo. O framework geral tem um objetivo mais ambicioso: gerar um único algoritmo capaz de ser efetivamente executado em várias bases de dados. O framework específico é testado em 20 bases públicas da UCI, e os resultados mostram que os algoritmos específicos gerados por HEAD-DT apresentam desempenho preditivo significativamente melhor do que algoritmos como CART e C4.5. O framework geral é executado em dois cenários diferentes: i) projeto de algoritmo específico a um domínio de aplicação; e ii) projeto de um algoritmo livre-de-domínio, robusto a bases distintas. O primeiro cenário é testado em 35 bases de expressão gênica, e os resultados mostram que o algoritmo gerado por HEAD-DT consistentemente supera CART e C4.5 em diferentes configurações experimentais. O segundo cenário é testado em 67 bases de dados da UCI, e os resultados mostram que o algoritmo gerado por HEAD-DT é competitivo com CART e C4.5. No entanto, é mostrado que HEAD-DT é vulnerável a um caso particular de overfitting quando executado sobre o segundo cenário do framework geral, e indica-se assim possíveis soluções para tal problema. Por fim, é realizado uma análise detalhada para avaliação de diferentes funções de fitness de HEAD-DT, onde 5 medidas de desempenho são combinadas com três esquemas de agregação. As 15 versões são avaliadas em 67 bases da UCI e as melhores versões são utilizadas para geração de algoritmos customizados para bases balanceadas e desbalanceadas. Os resultados mostram que os algoritmos gerados por HEAD-DT apresentam desempenho preditivo significativamente melhor que CART e C4.5, em uma clara indicação que HEAD-DT também é capaz de gerar algoritmos customizados para certo perfil estatístico dos dados de classificação. 



\section{Contents}

$\begin{array}{ll}\text { Acknowledgements } & \text { i }\end{array}$

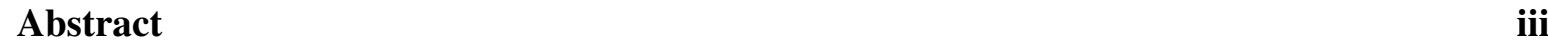

Resumo $\quad$ v

List of Figures

List of Tables $\quad$ xiii

List of Algorithms $\quad$ Xv

$\begin{array}{ll}\text { Notation } & \text { xvii }\end{array}$

1 Introduction 1

1.1 Thesis Statement $\ldots \ldots \ldots \ldots \ldots \ldots \ldots$

1.2 Thesis Contributions . . . . . . . . . . . . . . . 5

1.3 Informal Thesis Description $\ldots \ldots \ldots \ldots \ldots$

1.4 Thesis Outline . . . . . . . . . . . . . . . . . . . . . . . 7

2 Decision-Tree Induction 9

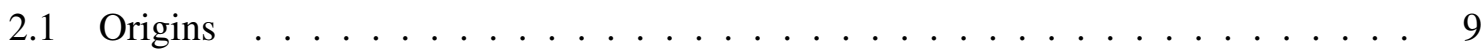

2.2 Basic Concepts . . . . . . . . . . . . . . . . . . . 10

2.3 Top-Down Induction $\ldots \ldots \ldots \ldots \ldots$

2.3 .1 Selecting Splits . . . . . . . . . . . . . . . . . . 13

2.3.2 Stopping Criteria . . . . . . . . . . . . . . . . 32

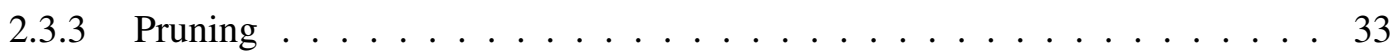

2.3 .4 Missing Values . . . . . . . . . . . . . . . . . . . . . . 39

2.4 Other Induction Strategies $\ldots \ldots \ldots \ldots$. . . . . . . . . . . . . 41 


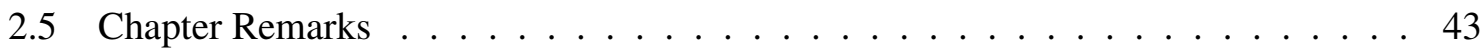

3 Evolutionary Algorithms and Hyper-Heuristics 45

3.1 Evolutionary Algorithms . . . . . . . . . . . . . . . . . . . 45

3.1.1 Individual Representation and Population Initialization . . . . . . . . . 47

3.1 .2 Fitness Function . . . . . . . . . . . . . . . . . . . . . . . 49

3.1.3 Selection Methods and Genetic Operators . . . . . . . . . . . . . . . . 50

3.2 Hyper-Heuristics . . . . . . . . . . . . . . . . . . . . 52

3.3 Chapter Remarks . . . . . . . . . . . . . . . . . . . . . . . . . 54

4 Evolutionary Algorithms for Decision-Tree Induction 55

4.1 Introduction . . . . . . . . . . . . . . . . . . . . 55

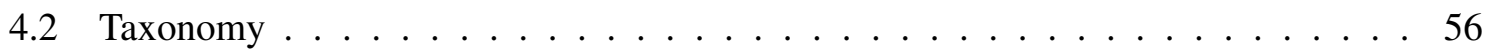

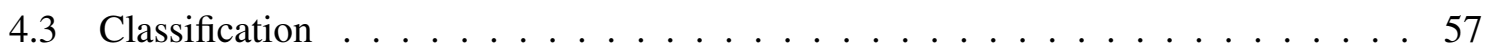

4.3.1 Axis-Parallel Decision Trees . . . . . . . . . . . . . . . . . . . . 57

4.3 .2 Oblique Decision Trees . . . . . . . . . . . . . . . . . . 70

4.4 Regression . . . . . . . . . . . . . . . . . . . . . . 73

4.4.1 Regression Trees . . . . . . . . . . . . . . . . . . 73

4.4 .2 Model Trees . . . . . . . . . . . . . . . . . . . . 76

4.5 Decision Tree-Components . . . . . . . . . . . . . . . . . . . . . 77

4.5 .1 Hyperplanes . . . . . . . . . . . . . . . . . . 77

4.5.2 Pruning Methods . . . . . . . . . . . . . . . . . . . 81

4.5.3 Other Decision-Tree Components _ . . . . . . . . . . . . . . . . 82

4.6 Applications of EAs for Decision-Tree Induction . . . . . . . . . . . . . . . 83

4.7 Chapter Remarks . . . . . . . . . . . . . . . . . . . . . . 85

4.7.1 The benefits of evolutionary decision trees . . . . . . . . . . . . 85

4.7.2 The drawbacks of evolutionary decision trees . . . . . . . . . . . 86

4.7.3 What is next on evolutionary induction of decision trees? . . . . . . . . 86

5 HEAD-DT: Automatic Design of Decision-Tree Algorithms 89

5.1 Introduction . . . . . . . . . . . . . . . . . . . . . . 90

5.2 Individual Representation . . . . . . . . . . . . . . . . . . . . . . . 91

5.2 .1 Split Genes . . . . . . . . . . . . . . . . . . . 91

5.2.2 Stopping Criteria Genes . . . . . . . . . . . . . . . . . . . 93

5.2 .3 Missing Values Genes . . . . . . . . . . . . . . . . . . . . . . . . . . . . . . . . . . 94

5.2 .4 Pruning Genes . . . . . . . . . . . . . . . . . . . . . 95

5.2.5 Example of Algorithm Evolved by HEAD-DT _ . . . . . . . . . . . . 97

5.3 Evolution . . . . . . . . . . . . . . . . . . . . . . 97

5.4 Fitness Evaluation . . . . . . . . . . . . . . . . . . . . . . . . 99

5.5 Search Space . . . . . . . . . . . . . . . . . . . . . 102 
5.6 Related Work . . . . . . . . . . . . . . . . . . . . . . . . . . 103

5.7 Chapter Remarks . . . . . . . . . . . . . . . . . . . . . . . . . . 104

6 HEAD-DT: Experimental Analysis 105

6.1 Evolving algorithms tailored to one specific data set . . . . . . . . . . . 106

6.2 Evolving algorithms from multiple data sets $\ldots \ldots \ldots \ldots \ldots$

6.2.1 The Homogeneous Approach $\ldots \ldots$. . . . . . . . . . . . . 111

6.2 .2 The Heterogeneous Approach $\ldots \ldots \ldots 122$

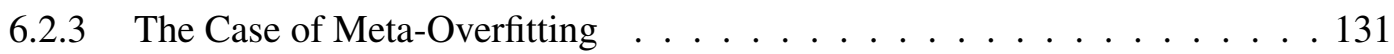

6.3 HEAD-DT's Time Complexity . . . . . . . . . . . . . . . . . . . . . 132

6.4 Cost-Effectiveness of Automated vs Manual Algorithm Design . . . . . . . . . 133

6.5 Examples of Automatically-Designed Algorithms . . . . . . . . . . . . . 134

6.6 Is the Genetic Search Worthwhile? . . . . . . . . . . . . . . . . . . . 136

6.7 Chapter Remarks . . . . . . . . . . . . . . . . . . . . . . . . . . . 140

7 HEAD-DT: Fitness Function Analysis 143

7.1 Performance Measures . . . . . . . . . . . . . . . . . . . . . . . . . . 144

7.1 .1 Accuracy . . . . . . . . . . . . . . . . . . . 144

7.1 .2 F-Measure . . . . . . . . . . . . . . . . . . . . 144

7.1.3 Area Under the ROC Curve . . . . . . . . . . . . . . . . . . . . . 145

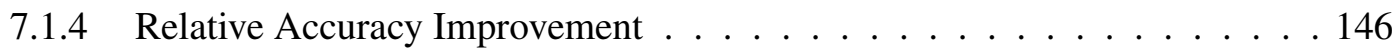

$7.1 .5 \quad$ Recall . . . . . . . . . . . . . . . . . . . . . . . . 146

7.2 Aggregation Schemes . . . . . . . . . . . . . . . . . . . . . . 147

7.3 Experimental Evaluation . . . . . . . . . . . . . . . . . . . . . . . . . 147

7.3.1 Results for the Balanced Meta-Training Set . . . . . . . . . . . . . 149

7.3.2 Results for the Imbalanced Meta-Training Set . . . . . . . . . . . . . 153

7.3.3 Experiments with the Best-Performing Strategy . . . . . . . . . . 160

7.4 Chapter Remarks . . . . . . . . . . . . . . . . . . . . . . 163

8 Conclusions 165

8.1 Limitations . . . . . . . . . . . . . . . . . . . . 166

8.2 Opportunities for Future Work . . . . . . . . . . . . . . . . . . . 167

8.2.1 Extending HEAD-DT's Genome: New Induction Strategies, Oblique Splits, Regression Problems . . . . . . . . . . . . . . . . 167

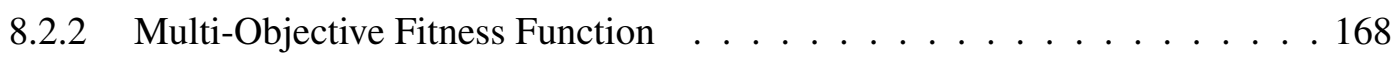

8.2.3 Automatic Selection of the Meta-Training Set . . . . . . . . . . . . 168

8.2.4 Parameter-Free Evolutionary Search . . . . . . . . . . . . . . . . . . . 169

8.2.5 Solving the Meta-Overfitting Problem . . . . . . . . . . . . . . . 169

8.2.6 Ensemble of Automatically-Designed Algorithms . . . . . . . . . . . . 170

8.2.7 Grammar-based Genetic Programming . . . . . . . . . . . . 170 
8.3 Publications . . . . . . . . . . . . . . . . . . . 170

$\begin{array}{ll}\text { References } & 177\end{array}$ 
2.1 Example of a general decision tree for classification.

2.2 A bivariate input space in a binary-class problem and its corresponding univariate decision tree. The single oblique split that separates the two classes is $x+y \leq 8$. Adapted from (Brodley and Utgoff, 1995). . . . . . . . . . . . . . . . . 28

2.3 (a) A bivariate input space in a binary-class problem: the spiral data set. (b) The corresponding axis-parallel partitioning. (c) The corresponding oblique partitioning. (d) The corresponding non-linear partitioning. Adapted from (Ittner, 1996). . . . . . . . . . . . . . . . . . . 31

2.4 Grafting of subtree rooted in 4 onto the place of parent 2. In (a) the original tree $T$ and in $(\mathrm{b})$ the pruned tree $T^{\prime} \ldots \ldots \ldots \ldots \ldots \ldots$

3.1 A GP individual represented as: a) a tree; b) a linear structure and c) a graph. . . . 48

3.2 Crossover strategies for linear strings: a) one-point crossover; b) two-point crossover; and c) uniform crossover. . . . . . . . . . . . . . . . . 51

3.3 Metaheuristics versus hyper-heuristics. . . . . . . . . . . . . . . . . . 53

4.1 Taxonomy of evolutionary algorithms for decision-tree induction. . . . . . . . . 57

4.2 Node representation in tree-encoding (Aitkenhead, 2008). A tuple of 4 elements that defines (1) the data set attribute to be tested or predicted; (2) the type of node (non-terminal or terminal); (3) the operator to be used; and (4) the value to be tested by the attribute in (1) according to the operator in (3) or, alternately, the binary classification value.

4.3 Mapping decision trees in linear chromosomes (Kennedy et al., 1997). a) decision tree. b) subtrees mapped into caltrops. c) chromosome resulting from the union of caltrops. . . . . . . . . . . . . . . . . . . 5 59

4.4 Initializing a hyperplane with randomly selected mixed dipole. Adapted from (Kretowski and Grzes, 2005). . . . . . . . . . . . . . . . . . . 72 
4.5 STGP regression tree example. Typed connections are presented. Adapted from (Hazan et al., 2006) . . . . . . . . . . . . . . . . . . . . . 75

5.1 HEAD-DT's evolutionary scheme. . . . . . . . . . . . . . . . . . . . . . 91

5.2 Linear-genome for evolving decision-tree algorithms. . . . . . . . . . . . . . . 91

5.3 HEAD-DT's genetic operators. . . . . . . . . . . . . . . . . . . 98

5.4 Evolution of individuals encoded as integer vectors. . . . . . . . . . . . . . . . 99

5.5 Fitness evaluation schemes. . . . . . . . . . . . . . . . . . . 100

6.1 Fitness evolution in HEAD-DT. . . . . . . . . . . . . . . . . . . . . . 119

6.2 Critical diagrams for the gene expression data. . . . . . . . . . . . . . . . 121

6.3 Critical diagrams for the UCI data sets. . . . . . . . . . . . . . . . . . 130

7.1 ROC curves for two different classifiers (Tan et al., 2005). . . . . . . . . . . . . 145

7.2 Critical diagrams for the balanced meta-training set experiment. . . . . . . . . . 152

7.3 Fitness evolution in HEAD-DT for the balanced meta-training set. . . . . . . . . . 154

7.4 Critical diagrams for the imbalanced meta-training set experiment. . . . . . . . . . 155

7.5 Fitness evolution in HEAD-DT for the imbalanced meta-training set. . . . . . . . . 159

7.6 Critical diagrams for accuracy and F-Measure. Values are regarding the 20 UCI data sets in Table 7.7. . . . . . . . . . . . . . . . . . . . . . . . . 162 
2.1 Univariate splitting criteria for classification. . . . . . . . . . . . . . . . . 27

2.2 Univariate splitting criteria for regression. . . . . . . . . . . . . . . . . 27

2.3 Multivariate splits. . . . . . . . . . . . . . . . 32

4.1 Application Areas of Evolutionary Decision Trees. . . . . . . . . . . . . . . 83

5.1 Split criteria implemented in HEAD-DT. . . . . . . . . . . . . . . . . . 92

5.2 Pruning methods implemented in HEAD-DT. . . . . . . . . . . . . . . 95

6.1 Summary of the data sets used in the experiments. . . . . . . . . . . . . . 106

6.2 Classification accuracy of CART, C4.5 and HEAD-DT. . . . . . . . . . . . . 108

6.3 Classification F-Measure of CART, C4.5 and HEAD-DT. . . . . . . . . . . . . . . 109

6.4 Tree size of CART, C4.5 and HEAD-DT trees. . . . . . . . . . . . . . . . . . 109

6.5 Summary of the 35 Gene Expression data sets. For each data set, we present type of microarray chip, the total number of instances, total number of attributes, imbalanced ratio (rate between over- and under-represented class), and total number of classes. . . . . . . . . . . . . . . . . . . . . . . . . . 112

6.6 Meta-training and meta-test configurations for the gene expression data. Data sets were randomly selected according to the methodology presented in the beginning of Section 6.2 . . . . . . . . . . . . . . . . . . . . . 113

6.7 Results of the tuning experiments for the homogeneous. HEAD-DT is executed with different values of parameter $p(\mathrm{H}-p)$. Values are the average performance (rank) of each HEAD-DT version in the corresponding meta-test set of tuning data sets, according to either accuracy or F-Measure . . . . . . . . . . . . . . . 114

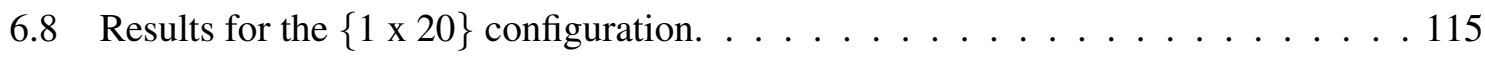

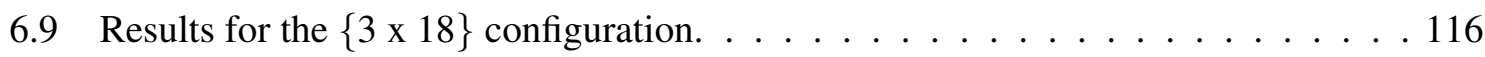

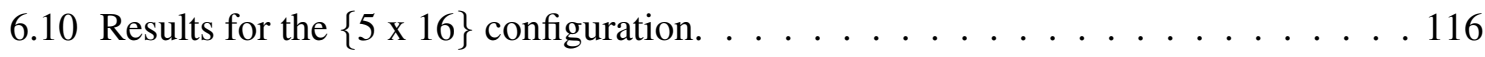

6.11 Results for the $\{7 \times 14\}$ configuration. . . . . . . . . . . . 117 
6.12 Results for the $\{9 \times 12\}$ configuration. . . . . . . . . . . . . . 117

6.13 Summary of the experimental analysis regarding the homogeneous approach. . . . 120

6.14 Summary of the 67 UCI data sets. . . . . . . . . . . . . . . . . . 123

6.15 Meta-training and meta-test configurations for the UCI data. . . . . . . . . . 124

6.16 Results of the tuning experiments for the heterogeneous approach. . . . . . . . . 125

6.17 Results for the $\{1 \times 39\}$ configuration. . . . . . . . . . . . 126

6.18 Results for the $\{3 \times 37\}$ configuration. . . . . . . . . . . . . . . 127

6.19 Results for the $\{5 \times 35\}$ configuration. . . . . . . . . . . . . . . . 127

6.20 Results for the $\{7 \times 33\}$ configuration. . . . . . . . . . . . . . 128

6.21 Results for the $\{9 \times 31\}$ configuration. . . . . . . . . . . . . 128

6.22 Summary of the experimental analysis regarding the heterogeneous approach. . . . 130

6.23 F-Measure achieved by HEAD-DT and C4.5 in the meta-training set. . . . . . . . . 132

6.24 HEAD-DT vs random search: $\{1 \times 39\}$ configuration. . . . . . . . . . . . . 137

6.25 HEAD-DT vs random search: $\{3 \times 37\}$ configuration. . . . . . . . . . . . . . 138

6.26 HEAD-DT vs random search: $\{5 \times 35\}$ configuration. . . . . . . . . . . . 138

6.27 HEAD-DT vs random search: $\{7 \times 33\}$ configuration. . . . . . . . . . . . . . . 139

6.28 HEAD-DT vs random search: $\{9 \times 31\}$ configuration. . . . . . . . . . . . . . 139

7.1 Accuracy values for the 15 versions of HEAD-DT varying the fitness functions. Meta-training comprises 5 balanced data sets. . . . . . . . . . . . 150

7.2 F-Measure values for the 15 versions of HEAD-DT varying the fitness functions. Meta-training comprises 5 balanced data sets. . . . . . . . . . . . . 151

7.3 Values are the average performance (rank) of each version of HEAD-DT according to either accuracy or F-Measure. . . . . . . . . . . . . . . . . . . 152

7.4 Accuracy values for the 15 versions of HEAD-DT varying the fitness functions. Meta-training comprises 5 imbalanced data sets. . . . . . . . . . . . . 156

7.5 F-Measure values for the 15 versions of HEAD-DT varying the fitness functions. Meta-training comprises 5 imbalanced data sets. . . . . . . . . . . . . . 157

7.6 Values are the average performance (rank) of each version of HEAD-DT according to either accuracy or F-Measure. . . . . . . . . . . . . . . . . . . 158

7.7 Accuracy and F-Measure values for the 10 most-balanced data sets and the 10 most-imbalanced data sets. . . . . . . . . . . . . . . . . . . . . 161 
1 Generic algorithmic framework for top-down induction of decision trees (Rokach and Maimon, 2005). Inputs are the training set $\mathbf{X}$, the predictive attribute set $A$ and the target attribute $y \ldots \ldots \ldots \ldots \ldots \ldots \ldots$

2 Deterministic OC1's procedure for perturbing a given coefficient. Parameters are the current hyperplane $H$ and the coefficient index $i \ldots \ldots \ldots$. . . . . . . 30

3 Pseudo-code for GP and GAs (Freitas, 2002) . . . . . . . . . . . . . . 46

4 Example of a decision-tree algorithm automatically designed by HEAD-DT. . . . . 97

5 Uniform crossover employed by HEAD-DT. . . . . . . . . . . . . . . . . . . . 98

6 Random uniform gene mutation employed by HEAD-DT. . . . . . . . . . . . . . . 98

7 Algorithm designed by HEAD-DT for the Semeion data set. . . . . . . . . . . . 135

8 Algorithm designed by HEAD-DT for the homogeneous approach (gene expression data), configuration $\{5 \times 16\} \ldots \ldots \ldots . \ldots \ldots 135$

9 Random search algorithm. . . . . . . . . . . . . . . . 136

10 Custom algorithm designed by HEAD-DT (RAI-M) for balanced data sets. . . . . 162

11 Custom algorithm designed by HEAD-DT (FM-A) for imbalanced data sets. . . . . 163 
|.| the cardinality of a given set

$T \quad$ a decision tree

$\mathrm{X} \quad$ a set of instances

$N_{x} \quad$ the number of instances in $\mathbf{X}$, i.e., $|\mathbf{X}|$

$\mathbf{x}^{j} \quad$ an instance - $n$-dimensional attribute vector $\left[x_{1}^{j}, x_{2}^{j}, \ldots, x_{n}^{j}\right]-$ from $\mathbf{X}, j=$ $1,2, \ldots, N_{x}$

$\mathbf{X}_{\mathbf{t}} \quad$ a set of instances that reach node $t$

$A \quad$ the set of $n$ predictive (independent) attributes $\left\{a_{1}, a_{2}, \ldots, a_{n}\right\}$

$y \quad$ the target (class) attribute

$Y \quad$ the set of $k$ class labels $\left\{y_{1}, \ldots, y_{k}\right\}$ (or $k$ distinct values if $y$ is continuous)

$y(x) \quad$ returns the class label (or target value) of instance $\mathbf{x} \in \mathbf{X}$

$a_{i}(x) \quad$ returns the value of attribute $a_{i}$ from instance $\mathbf{x} \in \mathbf{X}$

$\operatorname{dom}\left(a_{i}\right)$ the set of values attribute $a_{i}$ can take

$\left|a_{i}\right| \quad$ the number of partitions resulting from splitting attribute $a_{i}$

$\mathbf{X}_{\mathbf{a}_{\mathbf{i}}=\mathbf{v}_{\mathbf{j}}}$ the set of instances in which attribute $a_{i}$ takes a value contemplated by partition $v_{j}$. Edge $v_{j}$ can refer to a nominal value, to a set of nominal values or even to a numeric interval.

$N_{v_{j}, \bullet} \quad$ the number of instances in which attribute $a_{i}$ takes a value contemplated by partition $v_{j}$, i.e., $\left|\mathbf{X}_{\mathbf{a}_{\mathbf{i}}=\mathbf{v}_{\mathbf{j}}}\right|$

$\mathbf{X}_{\mathbf{y}=\mathbf{y}_{1}} \quad$ the set of instances in which the class attribute takes the label (value) $y_{l}$

$N_{\bullet}, y_{l} \quad$ the number of instances in which the class attribute takes the label (value) $y_{l}$, i.e., $\left|\mathbf{X}_{\mathbf{y}=\mathbf{y}_{\mathbf{1}}}\right|$

$N_{v_{j} \cap y_{l}} \quad$ the number of instances in which attribute $a_{i}$ takes a value contemplated by partition $v_{j}$ and in which the target attribute takes the label (value) $y_{l}$

$v_{X} \quad$ the target (class) vector $\left[N_{\bullet, y_{1}}, \ldots, N_{\bullet}, y_{k}\right]$ associated to $\mathbf{X}$

$p_{y} \quad$ the target (class) probability vector $\left[p_{\bullet}, y_{1}, \ldots, p_{\bullet}, y_{k}\right]$

$p_{\bullet}, y_{l} \quad$ the estimated probability of a given instance belonging to class $y_{l}$, i.e., $\frac{N_{\bullet}, y_{l}}{N_{x}}$ 
$p_{v_{j}} \bullet \quad$ the estimated probability of a given instance being contemplated by partition $v_{j}$, i.e., $\frac{N_{v_{j}, \bullet}}{N_{x}}$

$p_{v_{j} \cap y_{l}} \quad$ the estimated joint probability of a given instance being contemplated by partition $v_{j}$ and also belonging to class $y_{l}$, i.e., $\frac{N_{v_{j}} \cap y_{l}}{N_{x}}$

$p_{y_{l} \mid v_{j}} \quad$ the conditional probability of a given instance belonging to class $y_{l}$ given that it is contemplated by partition $v_{j}$, i.e., $\frac{N_{v_{j} \cap y_{l}}}{N_{v_{j}}, \bullet}$

$p_{v_{j} \mid y_{l}} \quad$ the conditional probability of a given instance being contemplated by partition $v_{j}$ given that it belongs to class $y_{l}$, i.e., $\frac{N_{v_{j}} \cap y_{l}}{N_{\bullet}, y_{l}}$

$\zeta_{T} \quad$ the set of non-terminal nodes in decision tree $T$

$\lambda_{T} \quad$ the set of terminal nodes in decision tree $T$

$\aleph_{T} \quad$ the set of nodes in decision tree $T$, i.e., $\aleph_{T}=\zeta_{T} \cup \lambda_{T}$

$T^{(t)} \quad$ a (sub)tree rooted in node $t$

$E^{(t)} \quad$ the number of instances in $t$ that do not belong to the majority class of that node 


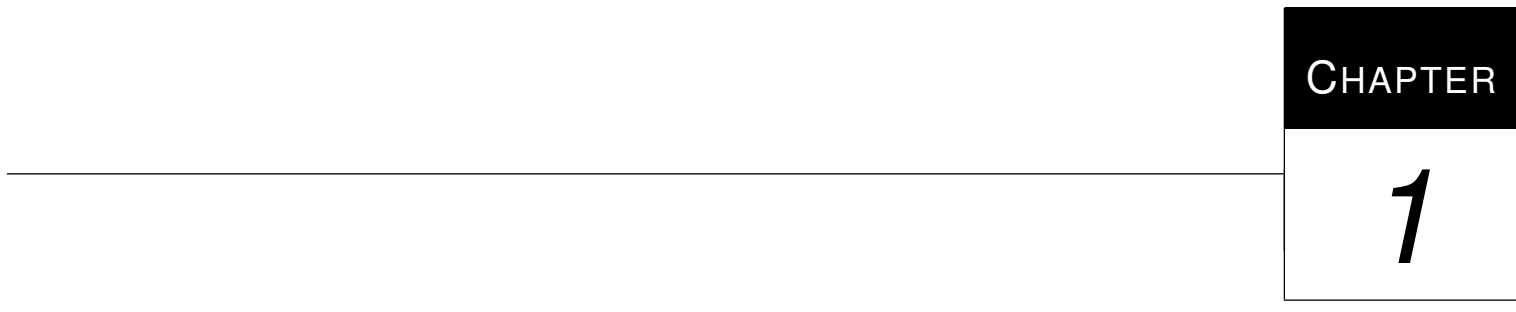

Introduction

Classification, which is the data mining task of assigning objects to predefined categories, is widely used in the process of intelligent decision making. Many classification techniques have been proposed by researchers in machine learning, statistics, and pattern recognition. Such techniques can be roughly divided according to the their level of comprehensibility. For instance, techniques that produce interpretable classification models are known as white-box approaches, whereas those that do not are known as black-box approaches. There are several advantages in employing white-box techniques for classification, such as increasing the user confidence in the prediction, providing new insight about the classification problem, and allowing the detection of errors either in the model or in the data (Freitas et al., 2010). Examples of white-box classification techniques are classification rules and decision trees. The latter is the main focus of this thesis.

A decision tree is a classifier represented by a flowchart-like tree structure that has been widely used to represent classification models, specially due to its comprehensible nature that resembles the human reasoning. In a recent poll from the kdnuggets website (KDNuggets, 2007), decision trees figured as the most used data mining/analytic method by researchers and practitioners, reaffirming its importance in machine learning tasks. Decision-tree induction algorithms present several advantages over other learning algorithms, such as robustness to noise, low computational cost for generating the model, and ability to deal with redundant attributes (Rokach and Maimon, 2005).

Several attempts on optimising decision-tree algorithms have been made by researchers within the last decades, even though the most successful algorithms date back to the mid-80's (Breiman et al., 1984) and early 90's (Quinlan, 1993). Many strategies were employed for deriving accurate decision trees, such as bottom-up induction (Barros et al., 2011b; Landeweerd et al., 1983), linear 
programming (Bennett and Mangasarian, 1994), hybrid induction (Kim and Landgrebe, 1991), and ensemble of trees (Breiman, 2001), just to name a few. Nevertheless, no strategy has been more successful in generating accurate and comprehensible decision trees with low computational effort than the greedy top-down induction strategy.

A greedy top-down decision-tree induction algorithm recursively analyses if a sample of data should be partitioned into subsets according to a given rule, or if no further partitioning is needed. This analysis takes into account a stopping criterion, for deciding when tree growth should halt, and a splitting criterion, which is responsible for choosing the "best" rule for partitioning a subset. Further improvements over this basic strategy include pruning tree nodes for enhancing the tree's capability of dealing with noisy data, and strategies for dealing with missing values, imbalanced classes, oblique splits, among others.

A very large number of approaches were proposed in the literature for each one of these design components of decision-tree induction algorithms. For instance, new measures for node-splitting tailored to a vast number of application domains were proposed, as well as many different strategies for selecting multiple attributes for composing the node rule (multivariate split). There are even studies in the literature that survey the numerous approaches for pruning a decision tree (Breslow and Aha, 1997; Esposito et al., 1997). It is clear that by improving these design components, more effective decision-tree induction algorithms can be obtained.

An approach that has been increasingly used in academia is the induction of decision trees through evolutionary algorithms (EAs). They are essentially algorithms inspired by the principle of natural selection and genetics. In nature, individuals are continuously evolving, adapting to their living environment. In EAs, each "individual" represents a candidate solution to the target problem. Each individual is evaluated by a fitness function, which measures the quality of its corresponding candidate solution. At each generation, the best individuals have a higher probability of being selected for reproduction. The selected individuals undergo operations inspired by genetics, such as crossover and mutation, producing new offspring which will replace the parents, creating a new generation of individuals. This process is iteratively repeated until a stopping criterion is satisfied (Eiben and Smith, 2008; Freitas, 2008). Instead of local search, EAs perform a robust global search in the space of candidate solutions. As a result, EAs tend to cope better with attribute interactions than greedy methods (Freitas, 2002).

The number of EAs for decision-tree induction has grown in the past few years, mainly because they report good predictive performance whilst keeping the comprehensibility of decision trees (Barros et al., 2012a). In this approach, each individual of the EA is a decision tree, and the evolutionary process is responsible for searching the solution space for the "near-optimal" tree regarding a given data set. A disadvantage of this approach is that it generates a decision tree tailored to a single data set. In other words, an EA has to be executed every time we want to induce a tree for a giving data set. Since the computational effort of executing an EA is much higher than executing the traditional greedy approach, it may not be the best strategy for inducing decision trees in time-constrained scenarios. 
Whether we choose to induce decision trees through the greedy strategy (top-down, bottom-up, hybrid induction), linear programming, EAs, ensembles, or any other available method, we are susceptible to the method's inductive bias. Since we know that certain inductive biases are more suitable to certain problems, and that no method is best for every single problem (i.e., the no free lunch theorem (Wolpert and Macready, 1997)), there is a growing interest in developing automatic methods for deciding which learner to use in each situation. A whole new research area named meta-learning has emerged for solving this problem (Smith-Miles, 2009). Meta-learning is an attempt to understand data a priori of executing a learning algorithm. In a particular branch of meta-learning, algorithm recommendation, data that describe the characteristics of data sets and learning algorithms (i.e., meta-data) are collected, and a learning algorithm is employed to interpret these meta-data and suggest a particular learner (or ranking a few learners) in order to better solve the problem at hand. Meta-learning has a few limitations, though. For instance, it provides a limited number of algorithms to be selected from a list. In addition, it is not an easy task to define the set of meta-data that will hopefully contain useful information for identifying the best algorithm to be employed.

For avoiding the limitations of traditional meta-learning approaches, a promising idea is to automatically develop algorithms tailored to a given domain or to a specific set of data sets. This approach can be seen as a particular type of meta-learning, since we are learning the "optimal learner" for specific scenarios. One possible technique for implementing this idea is genetic programming (GP). It is a branch of EAs that arose as a paradigm for evolving computer programs in the beginning of the 90's (Koza, 1992b). The idea is that each individual in GP is a computer program that evolves during the evolutionary process of the EA. Hopefully, at the end of evolution, GP will have found the appropriate algorithm (best individual) for the problem we want to solve. Pappa and Freitas (2009a) cite two examples of EA applications in which the evolved individual outperformed the best human-designed solution for the problem. In the first application (Keane and Brown, 1996), the authors designed a simple satellite dish holder boom (connection between the satellite's body and the communication dish) using an EA. This automatically designed dish holder boom, albeit its bizarre appearance, was shown to be $20,000 \%$ better than the human-designed shape. The second application (Oganov et al., 2009) was concerning the automatic discovery of a new form of boron (chemical element). There are only four known forms of borons, and the last one was discovered by an EA.

A recent research area within the combinatorial optimisation field named "hyper-heuristics" (HHs) has emerged with a similar goal: searching in the heuristics space, or in other words, heuristics to choose heuristics (Cowling et al., 2001a). HHs are related to metaheuristics, though with the difference that they operate on a search space of heuristics whereas metaheuristics operate on a search space of solutions to a given problem. Nevertheless, HHs usually employ metaheuristics (e.g., evolutionary algorithms) as the search methodology to look for suitable heuristics to a given problem (Pappa et al., 2013). Considering that an algorithm or its components 
can be seen as heuristics, one may say that HHs are also suitable tools to automatically design custom (tailor-made) algorithms.

Whether we name it "an EA for automatically designing algorithms" or "hyper-heuristics", in both cases there is a set of human designed components or heuristics, surveyed from the literature, which are chosen to be the starting point for the evolutionary process. The expected result is the automatic generation of new procedural components and heuristics during evolution, depending of course on which components are provided to the EA and the respective "freedom" it has for evolving the solutions.

The automatic design of complex algorithms is a much desired task by researchers. It was envisioned in the early days of artificial intelligence research, and more recently has been addressed by machine learning and evolutionary computation research groups (Pappa and Freitas, 2009a; Stanley and Miikkulainen, 2002; Vella et al., 2010). Automatically designing machine learning algorithms can be seen as the task of teaching the computer how to create programs that learn from experience. By providing an EA with initial human-designed programs, the evolutionary process will be in charge of generating new (and possibly better) algorithms for the problem at hand. Having said that, we believe an EA for automatically discovering new decision-tree induction algorithms may be the solution to avoid the drawbacks of the current decision-tree approaches, and this is going to be the main topic of this thesis.

\subsection{Thesis Statement}

The pioneering work by Pappa and Freitas (2006) on automatically evolving rule induction algorithms poses the following question: "if by changing these design components of rule induction algorithms can result in new, significantly better ones, why not keep on trying systematically?".

The question raised by Pappa and Freitas (2006) was answered by employing a grammar-based genetic programming approach for evolving full rule induction algorithms. The problem of automatically designing full rule induction algorithms is easily generalisable for decision-tree induction. More specifically, since the human manual approach for testing all possible modifications in the design components of decision-tree algorithms would be unfeasible, considering the vast amount of different strategies regarding each major design component, we formulate the following research hypothesis:

We can develop an EA capable of evolving and ultimately discovering new decision-tree induction algorithms able to outperform traditional human-designed ones. We can also evolve algorithms tailored to a particular domain, or to a particular statistical shape of data, by training this EA with suitable data sets. Such an approach may benefit from prior knowledge of the problem, e.g., current human-designed methods for decision-tree induction. 
The main objective of this thesis is similar to the one of Pappa and Freitas (2006): employing EAs to automatically design complex machine learning algorithms. We propose specifically evolving decision-tree induction algorithms, considering their importance in machine learning and data mining. Since each machine learning algorithm has an inductive bias, we expect evolution to be an appropriate strategy for finding the classifier whose bias is more suitable to the problem at hand. Other advantage of automatically designing algorithms is to avoid human biases and preferences. In principle, machine-designed algorithms could have very different biases from human-designed ones, which means they could excel at problems in which human-designed algorithms are not very effective (Pappa and Freitas, 2009a). Even though the proposed approach makes significant use of human-designed code, there is room for unexpected innovation, like shown in several EAs' applications (Keane and Brown, 1996; Oganov et al., 2009).

\subsection{Thesis Contributions}

When searching in the literature for related work, we found out that there are dozens of approaches that combine evolutionary algorithms and decision trees. Hence, we did a detailed survey of these approaches, presenting a taxonomy for dividing all work in the area. We also discussed in detail the advantages and disadvantages of employing evolutionary algorithms for generating decision trees, and pointed to future work in the area. This survey of evolutionary algorithms for decision-tree induction is presented in Chapter 4 of this thesis, being one of its specific contributions. Nevertheless, note that all work surveyed in Chapter 4 is about evolving decision trees and not decision-tree induction algorithms.

The main contribution of this thesis is a new algorithm for automatically design decision-tree induction algorithms. This new algorithm, presented in Chapter 5, makes use of an EA as its search methodology to evolve - and ultimately design — decision-tree algorithms for a given particular purpose. As specific contributions of this thesis regarding the automatic design of decision-tree algorithms, we can cite:

- The Specific Framework - Evolving a decision-tree induction algorithm tailored to one specific data set at a time. We show in Chapter 6, Section 6.1, that decision-tree algorithms that are designed to excel at a single data set usually outperform traditional decision-tree algorithms such as C4.5 (Quinlan, 1993) and CART (Breiman et al., 1984).

- The General Framework - Evolving a decision-tree induction algorithm from multiple data sets. We show in Chapters 6 and 7 that decision-tree algorithms may be designed to excel at a particular group of data sets, though with distinct objectives.

Specifically regarding the general framework, this thesis presents the following specific contributions: 
- Evolving a single decision-tree algorithm for data sets from a particular application domain. We perform a detailed empirical analysis on microarray gene expression data (Souto et al., 2008), and we show that automatically-designed decision-tree algorithms tailored to a particular domain usually outperform traditional decision-tree algorithms such as $\mathrm{C} 4.5$ and CART.

- Evolving a single decision-tree algorithm for a variety of data sets. We perform a thorough empirical analysis on almost 70 UCI data sets (Frank and Asuncion, 2010), and we show that automatically-designed decision-tree algorithms, which are meant to be robust across very different data sets, show a performance similar to traditional decision-tree algorithms such as C4.5 and CART.

- Evolving a single decision-tree algorithm for data sets with a particular statistical profile. After performing an extensive analysis on distinct fitness functions for HEAD-DT, we show that automatically-designed decision-tree algorithms tailored to balanced (and imbalanced) data sets usually outperform traditional decision-tree algorithms such as C4.5 and CART in these data sets.

Finally, we can include as specific contributions of this thesis a detailed discussion on the cost-effectiveness of automated algorithm design in contrast to the manual algorithm design, as well as an empirical demonstration confirming that the genetic search is significantly more effective than a random search in the space of decision-tree algorithms.

\subsection{Informal Thesis Description}

I decided to include this informal thesis description after being asked a hundred times "what is your thesis all about?", and failing to give a brief and proper answer to that question. If it is indeed true that Einstein once said that you should be able to explain anything to a 6-year-old (considering that you really know what you are talking about), then this is my attempt to informally explain my thesis to you, the interested reader.

This thesis is about making computers to automatically code (build, write, develop) computer programs. That is, to make them design a program without you specifically telling them what to do during the process. But not any kind of program, of course. It is about a very specific kind of computer program called "decision-tree induction algorithm".

But what is that and why should you care? Well, a decision tree is a graphical structure (like a flowchart) that is used to describe some amount of data. Imagine you work for a bank, and that this bank has stored data regarding its clients (the client profile information such as age, gender, income...) and also information on whether these clients paid off their loans or not. Now imagine a new client arrives asking you for a loan. Should you grant it or not? That's where a decision tree becomes handy. If you run a decision-tree algorithm over the bank's clients data, the computer 
program will provide you with typical profiles that are more likely to fail at paying their debts, as well as profiles that tend to pay off their debts as they should. For instance, the decision-tree algorithm may tell you that "if your client is between 20 and 30 years-old, has an annual income of $\$ 40,000$, and is divorced, then he/she is likely to pay off his/her debts". This is one of the possible paths a decision tree may give you depending on the historical data (we call it "training data") you provide to the decision-tree algorithm. Now imagine you have a decision tree with several of these rules (we call them paths, or branches). When your client arrives, all you have to do is follow the proper path and see what the decision tree recommends you to do! Easy, right?

"So how good are these decision trees?", you ask. Well they are good. Robust to several problems in the data. But there are of course many other computer programs that perform a similar task of labeling things (good/bad client, healthy/sick patient, etc.). For instance, perhaps you've heard about the famous neural networks. Or perhaps someone told you about SVMs, or ensembles. Well, why did I decide to work with decision trees and not all these other nice (and powerful!) techniques? Well, unlike decision trees, these techniques I've just cited do not tell you why you should grant the loan to your client, or why your patient is healthy or sick. They just say "grant the loan!”, or "patient is sick!". I don't know about you, but I prefer not to trust blindly in computers. It is much easier to trust someone when they tell you why you should follow their advice, so I guess the same thing applies to computers.

There, now you know what this thesis is about. Of course it is a little bit more than that, but hopefully you've got the general idea. "Did you manage to do what you're saying?", you ask. I think I did. I developed this program called HEAD-DT, and I showed that the decision-tree algorithms it generates are better than the cream of the crop of the existing decision-tree induction algorithms in several scenarios. I hope you enjoy the rest of this thesis as much as I enjoyed writing it.

\subsection{Thesis Outline}

This thesis is structured in 8 chapters. The remainder of this thesis is organized as follows.

Chapter 2 [Decision-Tree Induction] Origins, basic concepts, detailed components of top-down induction, and also other decision-tree induction strategies are presented.

Chapter 3 [Evolutionary Algorithms and Hyper-Heuristics] Origins, basic concepts, and techniques for both Evolutionary Algorithms and Hyper-Heuristics.

Chapter 4 [Evolutionary Algorithms for Decision-Tree Induction] A self-contained, detailed survey on studies that relate decision trees and evolutionary algorithms is presented. 
Chapter 5 [HEAD-DT: Automatic Design of Decision-Tree Induction Algorithms] The hyper-heuristic evolutionary algorithm that is capable of automatically designing decision-tree algorithms is introduced and discussed. Details such as the evolutionary scheme, building blocks, fitness evaluation, selection, genetic operators, and search space are presented in this chapter.

Chapter 6 [HEAD-DT: Experimental Analysis] A thorough empirical analysis on the distinct scenarios in which HEAD-DT may be applied to is performed. In addition, a discussion on the cost effectiveness of automatic design, as well as examples of automatically-designed algorithms and a baseline comparison between genetic and random search are also presented.

Chapter 7 [HEAD-DT: Fitness Function Analysis] A further investigation of 15 distinct versions of HEAD-DT varying its fitness functions is conducted, and a new set of experiments with the best-performing strategies in balanced and imbalanced data sets is performed.

Chapter 8 [Conclusions] Contributions, limitations, opportunities for future work, and publications are summarized, and the thesis is concluded. 


플

\section{Decision-Tree Induction}

Decision-tree induction algorithms are highly used in a variety of domains for knowledge discovery and pattern recognition. They have the advantage of producing a comprehensible classification/regression model and satisfactory accuracy levels in several application domains, such as medical diagnosis and credit risk assessment.

In this chapter, we present in detail the most common approach for decision-tree induction: top-down induction (Section 2.3). Furthermore, we briefly comment on some alternative strategies for induction of decision trees (Section 2.4). Our goal is to summarize the main design options one has to face when building decision-tree induction algorithms. These design choices will be specially interesting when designing an evolutionary algorithm for evolving decision-tree induction algorithms.

\subsection{Origins}

Automatically generating rules in the form of decision trees has been object of study of most research fields in which data exploration techniques have been developed (Murthy, 1998). Disciplines like engineering (pattern recognition), statistics, decision theory, and more recently artificial intelligence (machine learning) have a large number of studies dedicated to the generation and application of decision trees.

In statistics, we can trace the origins of decision trees to research that proposed building binary segmentation trees for understanding the relationship between target and input attributes. Some examples are AID (Sonquist et al., 1971), MAID (Gillo, 1972), THAID (Morgan and Messenger, 
1973), and CHAID (Kass, 1980). The application that motivated these studies is survey data analysis. In engineering (pattern recognition), research on decision trees was motivated by the need to interpret images from remote sensing satellites in the 70's (Hauska and Swain, 1975). Decision trees, and induction methods in general, arose in machine learning to avoid the knowledge acquisition bottleneck for expert systems (Murthy, 1998).

Specifically regarding top-down induction of decision trees (by far the most popular approach of decision-tree induction), Hunt's Concept Learning System (CLS) (Hunt et al., 1966) can be regarded as the pioneering work for inducing decision trees. Systems that directly descend from Hunt's CLS are ID3 (Quinlan, 1979), ACLS (Patterson and Niblett, 1983), and Assistant (Kononenko et al., 1984).

\subsection{Basic Concepts}

Decision trees are an efficient nonparametric method that can be applied either to classification or to regression tasks. They are hierarchical data structures for supervised learning whereby the input space is split into local regions in order to predict the dependent variable (Alpaydin, 2010).

A decision tree can be seen as a graph $G=(V, E)$ consisting of a finite, non-empty set of nodes (vertices) $V$ and a set of edges $E$. Such a graph has to satisfy the following properties (Safavian and Landgrebe, 1991):

- The edges must be ordered pairs $(v, w)$ of vertices, i.e., the graph must be directed;

- There can be no cycles within the graph, i.e., the graph must be acyclic;

- There is exactly one node, called the root, which no edges enter;

- Every node, except for the root, has exactly one entering edge;

- There is a unique path - a sequence of edges of the form $\left(v_{1}, v_{2}\right),\left(v_{2}, v_{3}\right), \ldots,\left(v_{n-1}, v_{n}\right)$ from the root to each node;

- When there is a path from node $v$ to $w, v \neq w, v$ is a proper ancestor of $w$ and $w$ is a proper descendant of $v$. A node with no proper descendant is called a leaf (or a terminal). All others are called internal nodes (except for the root).

Root and internal nodes hold a test over a given data set attribute (or a set of attributes), and the edges correspond to the possible outcomes of the test. Leaf nodes can either hold class labels (classification), continuous values (regression), (non-) linear models (regression), or even models produced by other machine learning algorithms. For predicting the dependent variable value of a certain instance, one has to navigate through the decision tree. Starting from the root, one has to follow the edges according to the results of the tests over the attributes. When reaching a leaf node, 


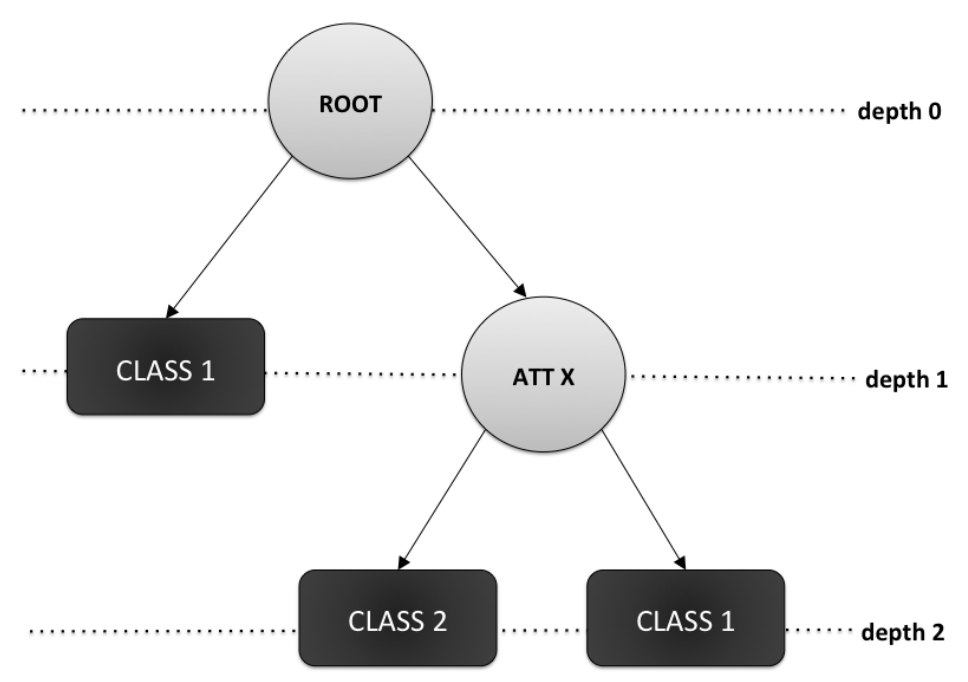

Figure 2.1: Example of a general decision tree for classification.

the information it contains is responsible for the prediction outcome. For instance, a traditional decision tree for classification holds class labels in its leaves.

Decision trees can be regarded as a disjunction of conjunctions of constraints on the attribute values of instances (Mitchell, 1997). Each path from the root to a leaf is actually a conjunction of attribute tests, and the tree itself allows the choice of different paths, that is, a disjunction of these conjunctions.

Other important definitions regarding decision trees are the concepts of depth and breadth. The average number of layers (levels) from the root node to the terminal nodes is referred to as the average depth of the tree. The average number of internal nodes in each level of the tree is referred to as the average breadth of the tree. Both depth and breadth are indicators of tree complexity, that is, the higher their values are, the more complex the corresponding decision tree is.

In Figure 2.1, an example of a general decision tree for classification is presented. Circles denote the root and internal nodes whilst squares denote the leaf nodes. In this particular example, the decision tree is designed for classification and thus the leaf nodes hold class labels.

There are many decision trees that can be grown from the same data. Induction of an optimal decision tree from data is considered to be a hard task. For instance, Hyafil and Rivest (1976) have shown that constructing a minimal binary tree with regard to the expected number of tests required for classifying an unseen object is an NP-complete problem. Hancock et al. (1996) have proved that finding a minimal decision tree consistent with the training set is NP-Hard, which is also the case of finding the minimal equivalent decision tree for a given decision tree (Zantema and Bodlaender, 2000), and building the optimal decision tree from decision tables (Naumov, 1991). These papers indicate that growing optimal decision trees (a brute-force approach) is only feasible in very small problems. 
Hence, it was necessary the development of heuristics for solving the problem of growing decision trees. In that sense, several approaches which were developed in the last three decades are capable of providing reasonably accurate, if suboptimal, decision trees in a reduced amount of time. Among these approaches, there is a clear preference in the literature for algorithms that rely on a greedy, top-down, recursive partitioning strategy for the growth of the tree (top-down induction).

\subsection{Top-Down Induction}

Hunt's Concept Learning System framework (CLS) (Hunt et al., 1966) is said to be the pioneer work in top-down induction of decision trees. CLS attempts to minimize the cost of classifying an object. Cost, in this context, is referred to two different concepts: the measurement cost of determining the value of a certain property (attribute) exhibited by the object, and the cost of classifying the object as belonging to class $j$ when it actually belongs to class $k$. At each stage, CLS exploits the space of possible decision trees to a fixed depth, chooses an action to minimize cost in this limited space, then moves one level down in the tree.

In a higher level of abstraction, Hunt's algorithm can be recursively defined in only two steps. Let $\mathbf{X}_{t}$ be the set of training instances associated with node $t$ and $y=\left\{y_{1}, y_{2}, \ldots, y_{k}\right\}$ be the class labels in a $k$-class problem (Tan et al., 2005):

1. If all the instances in $\mathbf{X}_{t}$ belong to the same class $y_{t}$ then $t$ is a leaf node labeled as $y_{t}$

2. If $\mathbf{X}_{t}$ contains instances that belong to more than one class, an attribute test condition is selected to partition the instances into smaller subsets. A child node is created for each outcome of the test condition and the instances in $\mathbf{X}_{t}$ are distributed to the children based on the outcomes. Recursively apply the algorithm to each child node.

Hunt's simplified algorithm is the basis for all current top-down decision-tree induction algorithms. Nevertheless, its assumptions are too stringent for practical use. For instance, it would only work if every combination of attribute values is present in the training data, and if the training data is inconsistency-free (each combination has a unique class label).

Hunt's algorithm was improved in many ways. Its stopping criterion, for example, as expressed in step 1, requires all leaf nodes to be pure (i.e., belonging to the same class). In most practical cases, this constraint leads to enormous decision trees, which tend to suffer from overfitting (an issue discussed later in this chapter). Possible solutions to overcome this problem include prematurely stopping the tree growth when a minimum level of impurity is reached, or performing a pruning step after the tree has been fully grown (more details on other stopping criteria and on pruning in Sections 2.3.2 and 2.3.3). Another design issue is how to select the attribute test condition to partition the instances into smaller subsets. In Hunt's original approach, a cost-driven function was responsible for partitioning the tree. Subsequent algorithms such as ID3 


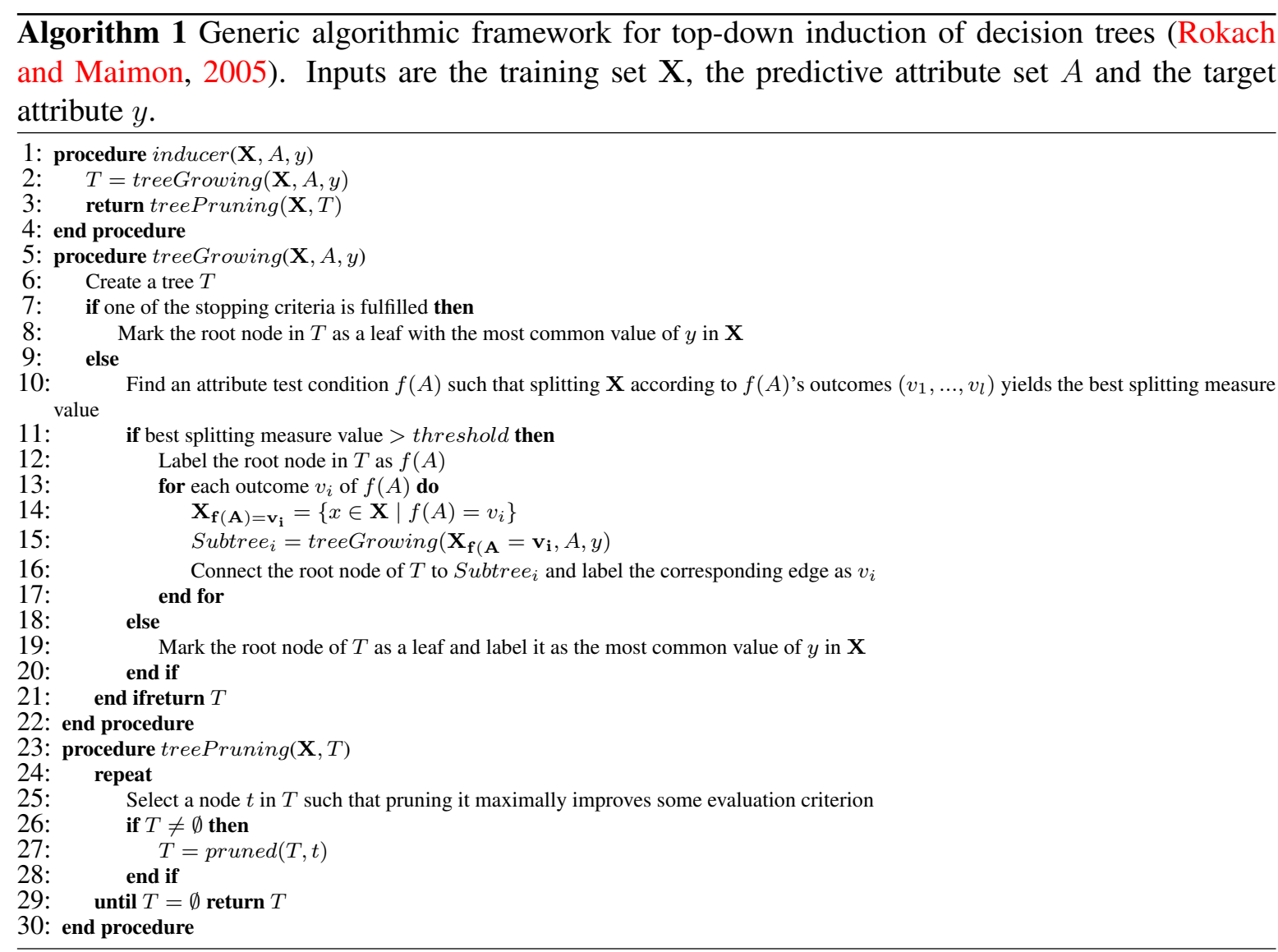

(Quinlan, 1979, 1986) and C4.5 (Quinlan, 1993) make use of information theory based functions for partitioning nodes in purer subsets (more details on Section 2.3.1).

An up-to-date algorithmic framework for top-down induction of decision trees is presented in (Rokach and Maimon, 2005), and we reproduce it in Algorithm 1. It contains three procedures: one for growing the tree (treeGrowing), one for pruning the tree (treePruning) and one to combine those two procedures (inducer). The first issue to be discussed is how to select the test condition $f(A)$, i.e., how to select the best combination of attribute(s) and value(s) for splitting nodes.

\subsubsection{Selecting Splits}

A major issue in top-down induction of decision trees is which attribute(s) to choose for splitting a node in subsets. For the case of axis-parallel decision trees (also known as univariate), the problem is to choose the attribute that better discriminates the input data. A decision rule based on such an attribute is thus generated, and the input data is filtered according to the outcomes of this rule. For oblique decision trees (also known as multivariate), the goal is to find a combination of attributes with good discriminatory power. Either way, both strategies are concerned with ranking attributes quantitatively.

We have divided the work in univariate criteria in the following categories: (i) information theory-based criteria; (ii) distance-based criteria; (iii) other classification criteria; and (iv) 
regression criteria. These categories are sometimes fuzzy and do not constitute a taxonomy by any means. Many of the criteria presented in a given category can be shown to be approximations of criteria in other categories.

\section{Information Theory-based Criteria}

Examples of this category are criteria based, directly or indirectly, on Shannon's entropy (Shannon, 1948). Entropy is known to be a unique function which satisfies the four axioms of uncertainty. It represents the average amount of information when coding each class into a codeword with ideal length according to its probability. Some interesting facts regarding entropy are:

- For a fixed number of classes, entropy increases as the probability distribution of classes becomes more uniform;

- If the probability distribution of classes is uniform, entropy increases logarithmically as the number of classes in a sample increases;

- If a partition induced on a set $\mathbf{X}$ by an attribute $a_{j}$ is a refinement of a partition induced by $a_{i}$, then the entropy of the partition induced by $a_{j}$ is never higher than the entropy of the partition induced by $a_{i}$ (and it is only equal if the class distribution is kept identical after partitioning). This means that progressively refining a set in sub-partitions will continuously decrease the entropy value, regardless of the class distribution achieved after partitioning a set.

The first splitting criterion that arose based on entropy is the global mutual information (GMI) (Gleser and Collen, 1972; Sethi and Sarvarayudu, 1982; Talmon, 1986), given by:

$$
G M I\left(a_{i}, \mathbf{X}, y\right)=\frac{1}{N_{x}} \sum_{l=1}^{k} \sum_{j=1}^{\left|a_{i}\right|} N_{v_{j} \cap y_{l}} \log _{e} \frac{N_{v_{j} \cap y_{l}} N_{x}}{N_{v_{j}, \bullet} N_{\bullet}, y_{l}}
$$

Ching et al. (1995) propose the use of GMI as a tool for supervised discretization. They name it class-attribute mutual information, though the criterion is exactly the same. GMI is bounded by zero (when $a_{i}$ and $y$ are completely independent) and its maximum value is $\max \left(\log _{2}\left|a_{i}\right|, \log _{2} k\right)$ (when there is a maximum correlation between $a_{i}$ and $y$ ). Ching et al. (1995) reckon this measure is biased towards attributes with many distinct values, and thus propose the following normalization called class-attribute interdependence redundancy (CAIR):

$$
\operatorname{CAIR}\left(a_{i}, \mathbf{X}, y\right)=\frac{G M I}{-\sum_{j=1}^{\left|a_{i}\right|} \sum_{l=1}^{k} p_{v_{j} \cap y_{l}} \log _{2} p_{v_{j} \cap y_{l}}}
$$


which is actually dividing GMI by the joint entropy of $a_{i}$ and $y$. Clearly $C A I R\left(a_{i}, \mathbf{X}, y\right) \geq 0$, since both GMI and the joint entropy are greater (or equal) than zero. In fact, $0 \leq C A I R\left(a_{i}, \mathbf{X}, y\right) \leq 1$, with $C A I R\left(a_{i}, \mathbf{X}, y\right)=0$ when $a_{i}$ and $y$ are totally independent and $C A I R\left(a_{i}, \mathbf{X}, y\right)=1$ when they are totally dependent. The term redundancy in CAIR comes from the fact that one may discretize a continuous attribute in intervals in such a way that the class-attribute interdependence is kept intact (i.e., redundant values are combined in an interval). In the decision tree partitioning context, we must look for an attribute that maximizes CAIR (or similarly, that maximizes GMI).

Information gain (Casey and Nagy, 1984; Hartmann et al., 1982; Quinlan, 1986; Varshney et al., 1982) is another example of measure based on Shannon's entropy. It belongs to the class of the so-called impurity-based criteria. The term impurity refers to the level of class separability among the subsets derived from a split. A pure subset is the one whose instances belong all to the same class. Impurity-based criteria are usually measures with values in $[0,1]$ where 0 refers to the purest subset possible and 1 the impurest (class values are equally distributed among the subset instances). More formally, an impurity-based criterion $\phi($.$) presents the following properties:$

- $\phi($.$) is minimum if \exists i$ such that $p_{\bullet}, y_{i}=1$;

- $\phi($.$) is maximum if \forall i, 1 \leq i \leq k, p_{\bullet}, y_{i}=1 / k$;

- $\phi($.$) is symmetric with respect to components of p_{y}$;

- $\phi($.$) is smooth (differentiable everywhere) in its range.$

Note that impurity-based criteria tend to favor a particular split for which, on average, the class distribution in each subset is most uneven. The impurity is measured before and after splitting a node according to each possible attribute. The attribute which presents the greater gain in purity, i.e., that maximizes the difference of impurity taken before and after splitting the node, is chosen. The gain in purity $(\Delta \Phi)$ can be defined as:

$$
\Delta \Phi\left(a_{i}, \mathbf{X}, y\right)=\phi(y, \mathbf{X})-\sum_{j=1}^{\left|a_{i}\right|} p_{v_{j}, \bullet} \times \phi\left(y, \mathbf{X}_{\mathbf{a}_{\mathbf{i}}=\mathbf{v}_{\mathbf{j}}}\right)
$$

The goal of information gain is to maximize the reduction in entropy due to splitting each individual node. Entropy can be defined as:

$$
\phi^{\text {entropy }}(\mathbf{X}, y)=-\sum_{l=1}^{k} p_{\bullet}, y_{l} \times \log _{2} p_{\bullet}, y_{l} .
$$

If entropy is calculated in (2.3), then $\Delta \Phi\left(a_{i}, \mathbf{X}\right)$ is the information gain measure, which calculates the goodness of splitting the instance space $\mathbf{X}$ according to the values of attribute $a_{i}$. 
Wilks (1962) has proved that as $N \rightarrow \infty, 2 \times N_{x} \times G M I\left(a_{i}, \mathbf{X}, y\right)$ (or similarly replacing GMI by information gain) approximate the $\chi^{2}$ distribution. This measure is often regarded as the G statistics (Mingers, 1987, 1989b). White and Liu (1994) point out that the G statistics should be adjusted since the work of Mingers (1989b) uses logarithms to base $e$, instead of logarithms to base 2 . The adjusted $\mathrm{G}$ statistics is given by $2 \times N_{x} \times \Delta \Phi^{I G} \times \log _{e} 2$. Instead of using the value of this measure as calculated, we can compute the probability of such a value occurring from the $\chi^{2}$ distribution on the assumption that there is no association between the attribute and the classes. The higher the calculated value, the less likely it is to have occurred given the assumption. The advantage of using such a measure is making use of the levels of significance it provides for deciding whether to include an attribute at all.

Quinlan (1986) acknowledges the fact that the information gain is biased towards attributes with many values. This is a consequence of the previously mentioned particularity regarding entropy, in which further refinement leads to a decrease in its value. Quinlan proposes a solution for this matter called gain ratio (Quinlan, 1993). It basically consists of normalizing the information gain by the entropy of the attribute being tested, that is,

$$
\Delta \Phi^{\text {gainRatio }}\left(a_{i}, \mathbf{X}, y\right)=\frac{\Delta \Phi^{I G}}{\phi^{\text {entropy }}\left(a_{i}, \mathbf{X}\right)}
$$

The gain ratio compensates the decrease in entropy in multiple partitions by dividing the information gain by the attribute self-entropy $\phi^{\text {entropy }}\left(a_{i}, \mathbf{X}\right)$. The value of $\phi^{\text {entropy }}\left(a_{i}, \mathbf{X}\right)$ increases logarithmically as the number of partitions over $a_{i}$ increases, decreasing the value of gain ratio. Nevertheless, the gain ratio has two deficiencies: (i) it may be undefined (i.e., the value of self-entropy may be zero); and (ii) it may choose attributes with very low self-entropy but not with high gain. For solving these issues, Quinlan suggests first calculating the information gain for all attributes, and then calculating the gain ratio only for those cases in which the information gain value is above the average value of all attributes.

Several variations of the gain ratio have been proposed. For instance, the normalized gain (Jun et al., 1997) replaces the denominator of gain ratio by $\log _{2}\left|a_{i}\right|$. The authors demonstrate two theorems with cases in which the normalized gain works better than or at least equally as either information gain or gain ratio does. In the first theorem, they prove that if two attributes $a_{i}$ and $a_{j}$ partition the instance space in pure sub-partitions, and that if $\left|a_{i}\right|>\left|a_{j}\right|$, normalized gain will always prefer $a_{j}$ over $a_{i}$, whereas gain ratio is dependent of the self-entropy values of $a_{i}$ and $a_{j}$ (which means gain ratio may choose the attribute that partitions the space in more values). The second theorem states that given two attributes $a_{i}$ and $a_{j},\left|a_{i}\right|=\left|a_{j}\right|,\left|a_{i}\right| \geq 2$, if $a_{i}$ partitions the instance space in pure subsets and $a_{j}$ has at least one subset with more than one class, normalized gain will always prefer $a_{i}$ over $a_{j}$, whereas gain ratio will prefer $a_{j}$ if the following condition is met: 


$$
\begin{gathered}
\frac{E\left(a_{j}, \mathbf{X}, y\right)}{\phi^{\text {entropy }}(y, \mathbf{X})} \leq 1-\frac{\phi^{\text {entropy }}\left(a_{j}, \mathbf{X}\right)}{\phi^{\text {entropy }}\left(a_{i}, \mathbf{X}\right)} \\
\text { where: } \\
E\left(a_{j}, \mathbf{X}, y\right)=-\sum_{l=1}^{\left|a_{j}\right|} p_{v_{l}, \bullet} \times \phi^{\text {entropy }}\left(y, \mathbf{X}_{\mathbf{a}_{\mathbf{j}}=\mathbf{v}_{\mathbf{l}}}\right)
\end{gathered}
$$

For details on the proof of each theorem, please refer to Jun et al. (1997).

Other variation is the average gain (Wang and Jiang, 2007), that replaces the denominator of gain ratio by $\left|\operatorname{dom}\left(a_{i}\right)\right|$ (it only works for nominal attributes). The authors do not demonstrate theoretically any situations in which this measure is a better option than gain ratio. Their work is supported by empirical experiments in which the average gain outperforms gain ratio in terms of runtime and tree size, though with no significant differences regarding accuracy. Note that most decision-tree induction algorithms provide one branch for each nominal value an attribute can take. Hence, the average gain (Wang and Jiang, 2007) is practically identical to the normalized gain (Jun et al., 1997), though without scaling the number of values with $\log _{2}$.

Sá et al. (2009) propose a somewhat different splitting measure based on the minimum entropy of error principle (MEE) (Silva et al., 2006). It does not directly depend on the class distribution of a node $p_{v_{j}, y_{l}}$ and the prevalences $p_{v_{j}, \bullet}$, but instead it depends on the errors produced by the decision rule on the form of a Stoller split (Devroye et al., 1996): if $a_{i}(x) \leq \Delta, y(x)=y_{\omega} ; \hat{y}$ otherwise. In a Stoller split, each node split is binary and has an associated class $y_{\omega}$ for the case $a_{i}(x) \leq \Delta$, while the remaining classes are denoted by $\hat{y}$ and associated to the complementary branch. Each class is assigned a code $t \in\{-1,1\}$, in such a way that for $y(x)=y_{\omega}, t=1$ and for $y(x)=\hat{y}, t=-1$. The splitting measure is thus given by:

$$
\begin{gathered}
\operatorname{MEE}\left(a_{i}, \mathbf{X}, y\right)=-\left(P_{-1} \log _{e} P_{-1}+P_{0} \log _{e} P_{0}+P_{1} \log _{e} P_{1}\right) \\
\text { where: } \\
P_{-1}=\frac{N_{\bullet}, y_{l}}{n} \times \frac{e_{1,-1}}{N_{x}} \\
P_{1}=\left(1-\frac{N_{\bullet}, y_{l}}{n}\right) \times \frac{e_{-1,1}}{N_{x}} \\
P_{0}=1-P_{-1}-P_{1}
\end{gathered}
$$

where $e_{t, t^{\prime}}$ is the number of instances $t$ classified as $t^{\prime}$. Note that unlike other measures such as information gain and gain ratio, there is no need of computing the impurity of sub-partitions and their subsequent average, as MEE does all the calculation needed at the current node to be split. MEE is bounded by the interval $\left[0, \log _{e} 3\right]$, and needs to be minimized. The meaning of minimizing MEE is constraining the probability mass function of the errors to be as narrow as possible (around 
zero). The authors argue that by using MEE, there is no need of applying the pruning operation, saving execution time of decision-tree induction algorithms.

\section{Distance-based Criteria}

Criteria in this category evaluate separability, divergency or discrimination between classes. They measure the distance between class probability distributions.

A popular distance criterion which is also from the class of impurity-based criteria is the Gini index (Breiman et al., 1984; Gelfand et al., 1989; Pattipati and Alexandridis, 1990). It is given by:

$$
\phi^{\operatorname{Gini}}(y, \mathbf{X})=1-\sum_{l=1}^{k} p_{\bullet}, y_{l}{ }^{2}
$$

Breiman et al. (1984) also acknowledge Gini's bias towards attributes with many values. They propose the twoing binary criterion for solving this matter. It belongs to the class of binary criteria, which requires attributes to have their domain split into two mutually exclusive subdomains, allowing binary splits only. For every binary criteria, the process of dividing attribute $a_{i}$ values into two subdomains, $d_{1}$ and $d_{2}$, is exhaustive ${ }^{1}$ and the division that maximizes its value is selected for attribute $a_{i}$. In other words, a binary criterion $\beta$ is tested over all possible subdomains in order to provide the optimal binary split, $\beta^{*}$ :

$$
\begin{aligned}
& \beta^{*}=\max _{d_{1}, d_{2}} \beta\left(a_{i}, d_{1}, d_{2}, \mathbf{X}, y\right) \\
& \text { s.t. } \\
& d_{1} \cup d_{2}=\operatorname{dom}\left(a_{i}\right) \\
& d_{1} \cap d_{2}=\emptyset
\end{aligned}
$$

Now that we have defined binary criteria, the twoing binary criterion is given by:

$$
\begin{gathered}
\beta^{\text {twoing }}\left(a_{i}, d_{1}, d_{2}, \mathbf{X}, y\right)=0.25 \times p_{d_{1}, \bullet} \times p_{d_{2}, \bullet} \times \\
\left(\sum_{l=1}^{k} a b s\left(p_{y_{l} \mid d_{1}}-p_{y_{l} \mid d_{2}}\right)\right)^{2}
\end{gathered}
$$

where $a b s($.$) returns the absolute value.$

\footnotetext{
${ }^{1}$ Coppersmith et al. (1999) present an interesting heuristic procedure for finding subdomains $d_{1}$ and $d_{2}$ when the partition is based on a nominal attribute. Shih (2001) also investigates the problem of efficiency on finding the best binary split for nominal attributes.
} 
Friedman (1977) and Rounds (1980) propose a binary criterion based on the Kolmogorov-Smirnoff (KS) distance for handling binary-class problems:

$$
\beta^{K S}\left(a_{i}, d_{1}, d_{2}, \mathbf{X}, y\right)=\operatorname{abs}\left(p_{d_{1} \mid y_{1}}-p_{d_{1} \mid y_{2}}\right)
$$

Haskell and Noui-Mehidi (1991) propose extending $\beta^{K S}$ for handling multi-class problems. Utgoff and Clouse (1996) also propose a multi-class extension to $\beta^{K S}$, as well as missing data treatment, and they present empirical results which show their criterion is similar in accuracy to Quinlan's gain ratio, but produces smaller-sized trees.

The $\chi^{2}$ statistic (Mingers, 1989b; White and Liu, 1994; Zhou and Dillon, 1991) has been employed as a splitting criterion in decision trees. It compares the observed values with those that one would expect if there were no association between attribute and class. The resulting statistic is distributed approximately as the chi-square distribution, with larger values indicating greater association. Since we are looking for the predictive attribute with the highest degree of association to the class attribute, this measure must be maximized. It can be calculated as:

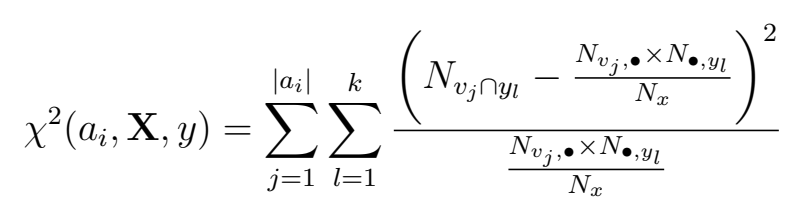

It should be noticed that the $\chi^{2}$ statistic (and similarly the $\mathrm{G}$ statistic) become poor approximations with small expected frequencies. Small frequencies make $\chi^{2}$ over-optimistic in detecting informative attributes, i.e., the probability derived from the distribution will be smaller than the true probability of getting a value of $\chi^{2}$ as large as that obtained.

De Mántaras (1991) proposes a distance criterion that "provides a clearer and more formal framework for attribute selection and solves the problem of bias in favor of multivalued attributes without having the limitations of Quinlan's Gain Ratio". It is actually the same normalization to information gain as CAIR is to GMI, i.e.,

$$
1-\Delta \Phi^{\text {distance }}\left(a_{i}, \mathbf{X}, y\right)=\frac{\Delta \Phi^{I G}}{-\sum_{j=1}^{\left|a_{i}\right|} \sum_{l=1}^{k} p_{v_{j} \cap y_{l}} \log _{2} p_{v_{j} \cap y_{l}}} .
$$

Notice that in (2.13), we are actually presenting the complement of Mántara's distance measure, i.e., $1-\Delta \Phi^{\text {distance }}\left(a_{i}, \mathbf{X}, y\right)$, but with no implications in the final result (apart from the fact that (2.13) needs to be maximized, whereas the original distance measure should be minimized).

Fayyad and Irani (1992) propose a new family of measures called C-SEP (from Class SEParation). They claim that splitting measures such as information gain (and similar impurity-based criteria) suffer from a series of deficiencies (e.g., they are insensitive to within-class 
fragmentation), and they present new requirements a "good" splitting measure $\Gamma($.$) (in particular,$ binary criteria) should fulfill:

- $\Gamma($.$) is maximum when classes in d_{1}$ and $d_{2}$ are disjoint (inter-class separability);

- $\Gamma($.$) is minimum when the class distributions in d_{1}$ and $d_{2}$ are identical;

- $\Gamma$ (.) favors partitions which keep instances from the same class in the same subdomain $d_{i}$ (intra-class cohesiveness);

- $\Gamma($.$) is sensitive to permutations in the class distribution;$

- $\Gamma($.$) is non-negative, smooth (differentiable), and symmetric with respect to the classes.$

Binary criteria that fulfill the above requirements are based on the premise that a good split is the one that separates as many different classes from each other as possible, while keeping examples of the same class together. $\Gamma$ must be maximized, unlike the previously presented impurity-based criteria.

Fayyad and Irani (1992) propose a new binary criterion from this family of C-SEP measures called $O R T$, defined as:

$$
\begin{aligned}
& \Gamma^{O R T}\left(a_{i}, d_{1}, d_{2}, \mathbf{X}, y\right)=1-\theta\left(v_{d_{1}}, v_{d_{2}}\right) \\
& \theta\left(v_{d_{1}}, v_{d_{2}}\right)=\frac{v_{d_{1}} \cdot v_{d_{2}}}{\left\|v_{d_{1}}\right\| \times\left\|v_{d_{2}}\right\|}
\end{aligned}
$$

where $v_{d_{i}}$ is the class vector of the set of instances $\mathbf{X}_{\mathbf{i}}=\left\{x \in \mathbf{X} \mid \mathbf{X}_{\mathbf{a}_{\mathbf{i}} \in \mathbf{d}_{\mathbf{i}}}\right\}$, "." represents the inner product between two vectors and $\|$.$\| the magnitude (norm) of a vector. Note that O R T$ is basically the complement of the well-known cosine distance, which measures the orthogonality between two vectors. When the angle between two vectors is $90^{\circ}$, it means the non-zero components of each vector do not overlap. The $O R T$ criterion is maximum when the cosine distance is minimum, i.e., the vectors are orthogonal, and it is minimum when they are parallel. The higher the values of $O R T$, the greater the distance between components of the class vectors (maximum ORT means disjoint classes).

Taylor and Silverman (1993) propose a splitting criterion called mean posterior improvement (MPI), which is given by:

$$
\beta^{M P I}\left(a_{i}, d_{1}, d_{2}, \mathbf{X}, y\right)=p_{d_{1}, \bullet} p_{d_{2}, \bullet}-\sum_{l=1}^{k}\left[p_{\bullet}, y_{l} p_{d_{1} \cap y_{l}} p_{d_{2} \cap y_{l}}\right]
$$

The MPI criterion provides maximum value when individuals of the same class are all placed in the same partition, and thus, (2.15) should be maximized. Classes over-represented in the father node will have a greater emphasis in the MPI calculation (such an emphasis is given by the $p_{\bullet}, y_{l}$ 
in the summation). The term $p_{d_{1} \cap y_{l}} p_{d_{2} \cap y_{l}}$ is desired to be small since the goal of MPI is to keep instances of the same class together and to separate them from those of other classes. Hence, $p_{d_{1}, \bullet} p_{d_{2}, \bullet}-p_{d_{1} \cap y_{l}} p_{d_{2} \cap y_{l}}$ is the improvement that the split is making for class $y_{l}$, and therefore the MPI criterion is the mean improvement over all the classes.

Mola and Siciliano (1997) propose using the predictability index $\tau$ originally proposed in (Goodman and Kruskal, 1954) as a splitting measure. The $\tau$ index can be used first to evaluate each attribute individually (2.16), and then to evaluate each possible binary split provided by grouping the values of a given attribute in $d_{1}$ and $d_{2}$ (2.17).

$$
\begin{gathered}
\beta^{\tau}\left(a_{i}, \mathbf{X}, y\right)=\frac{\sum_{j=1}^{\left|a_{i}\right|} \sum_{l=1}^{k}\left(p_{v_{j} \cap y_{l}}\right)^{2} \times p_{v_{j}, \bullet}-\sum_{l=1}^{k} p_{\bullet, y_{l}}^{2}}{1-\sum_{l=1}^{k} p_{\bullet, y_{l}}^{2}} \\
\beta^{\tau}\left(a_{i}, d_{1}, d_{2}, \mathbf{X}, y\right)=\frac{\sum_{l=1}^{k}\left(p_{d_{1} \cap y_{l}}\right)^{2} p_{d_{1}, \bullet}+\sum_{l=1}^{k}\left(p_{d_{2} \cap y_{l}}\right)^{2} p_{d_{2}, \bullet}-\sum_{l=1}^{k} p_{\bullet, y_{l}}^{2}}{1-\sum_{j=1}^{k} p_{y_{j}}^{2}}
\end{gathered}
$$

Now, consider that $\beta^{\tau *}\left(a_{i}\right)=\max _{d_{1}, d_{2}} \beta^{\tau}\left(a_{i}, d_{1}, d_{2}, \mathbf{X}, y\right)$. Mola and Siciliano (1997) prove a theorem saying that

$$
\begin{gathered}
\beta^{\tau}\left(a_{i}, \mathbf{X}, y\right) \geq \beta^{\tau}\left(a_{i}, d_{1}, d_{2}, \mathbf{X}, y\right) \\
\text { and also that } \\
\beta^{\tau}\left(a_{i}, \mathbf{X}, y\right) \geq \beta^{\tau *}\left(a_{i}\right) .
\end{gathered}
$$

This theoretical evidence is of great importance for providing a means to select the best attribute and its corresponding binary partitions without the need of exhaustively trying all possibilities. More specifically, one has to calculate (2.16) for all attributes, and to sort them according to the highest values of $\beta^{\tau}(*, \mathbf{X}, y)$, in such a way that $a_{1}$ is the attribute that yields the highest value of $\beta^{\tau}(*, \mathbf{X}, y), a_{2}$ is the second highest value, and so on. Then, one has to test all possible splitting options in (2.17) in order to find $\beta^{\tau *}\left(a_{1}\right)$. If the value of $\beta^{\tau *}\left(a_{1}\right)$ is greater than the value of $\beta^{\tau}\left(a_{2}, \mathbf{X}, y\right)$, we do not need to try any other split possibilities, since we know that $\beta^{\tau *}\left(a_{2}\right)$ is necessarily lesser than $\beta^{\tau}\left(a_{2}, \mathbf{X}, y\right)$. For a simple but efficient algorithm implementing this idea, please refer to the appendix in (Mola and Siciliano, 1997).

\section{Other Classification Criteria}

In this category, we include all criteria that did not fit in the previously-mentioned categories.

Li and Dubes (1986) propose a binary criterion for binary-class problems called permutation statistic. It evaluates the degree of similarity between two vectors, $V_{a_{i}}$ and $y$, and the larger 
this statistic, the more alike the vectors. Vector $V_{a_{i}}$ is calculated as follows. Let $a_{i}$ be a given numeric attribute with the values $[8.20,7.3,9.35,4.8,7.65,4.33]$ and $N_{x}=6$. Vector $y=[0,0,1,1,0,1]$ holds the corresponding class labels. Now consider a given threshold $\Delta=5.0$. Vector $V_{a_{i}}$ is calculated in two steps: first, attribute $a_{i}$ values are sorted, i.e., $a_{i}=[4.33,4.8,7.3,7.65,8.20,9.35]$, consequently rearranging $y=[1,1,0,0,0,1]$; then, $V_{a_{i}}(n)$ takes 0 when $a_{i}(n) \leq \Delta$, and 1 otherwise. Thus, $V_{a_{i}}=[0,0,1,1,1,1]$. The permutation statistic first analyses how many 1-1 matches $(d)$ vectors $V_{a_{i}}$ and $y$ have. In this particular example, $d=1$. Next, it counts how many 1's there are in $V_{a_{i}}\left(n_{a}\right)$ and in $y\left(n_{y}\right)$. Finally, the permutation statistic can be computed as:

$$
\begin{aligned}
\beta^{\text {permutation }}\left(V_{a_{i}}, y\right) & =\sum_{j=0}^{d} \frac{\left(\begin{array}{c}
n_{a} \\
j
\end{array}\right)\left(\begin{array}{c}
N_{x}-n_{a} \\
n_{y}-j
\end{array}\right)}{\left(\begin{array}{c}
N_{x} \\
n_{y}
\end{array}\right)}-\frac{\left(\begin{array}{c}
n_{a} \\
d
\end{array}\right)\left(\begin{array}{c}
N_{x}-n_{a} \\
n_{y}-d
\end{array}\right)}{\left(\begin{array}{c}
N_{x} \\
n_{y}
\end{array}\right)} U \\
\left(\begin{array}{c}
n \\
m
\end{array}\right) & =0 \text { if } n<0 \text { or } m<0 \text { or } n<m \\
& =\frac{n !}{m !(n-m) !} \text { otherwise }
\end{aligned}
$$

where $U$ is a (continuous) random variable distributed uniformly over $[0,1]$.

The permutation statistic presents an advantage over the information gain and other criteria: it is not sensitive to the data fragmentation problem ${ }^{2}$. It automatically adjusts for variations in the number of instances from node to node because its distribution does not change with the number of instances at each node.

Quinlan and Rivest (1989) propose using the minimum description length principle (MDL) as a splitting measure for decision-tree induction. MDL states that, given a set of competing hypotheses (in this case, decision trees), one should choose as the preferred hypothesis the one that minimizes the sum of two terms: (i) the description length of the hypothesis $\left(d_{l}\right)$; and (ii) length of the data given the hypothesis $\left(l_{h}\right)$. In the context of decision trees, the second term can be regarded as the length of the exceptions, i.e., the length of certain objects of a given subset whose class value is different from the most frequent one. Both terms are measured in bits, and thus one needs to encode the decision tree and exceptions accordingly.

It can be noticed that by maximizing $d_{l}$, we minimize $l_{h}$, and vice-versa. For instance, when we grow a decision tree until each node has objects that belong to the same class, we usually end up with a large tree (maximum $d_{l}$ ) prone to overfitting, but with no exceptions (minimum $l_{h}$ ). Conversely, if we allow a large number of exceptions, we will not need to partition subsets any further, and in the extreme case (maximum $l_{h}$ ), the decision tree will hold a single leaf node

\footnotetext{
${ }^{2}$ Data fragmentation is a well-known problem in top-down decision trees. Nodes with few instances usually lack statistical support for further partitioning. This phenomenon happens for most of the split criteria available, since their distributions depend on the number of instances in each particular node.
} 
labeled as the most frequent class value (minimum $d_{l}$ ). Hence the need of minimizing the sum $d_{l}+l_{h}$.

MDL provides a way of comparing decision trees once the encoding techniques are chosen. Finding a suitable encoding scheme is usually a very difficult task, and the values of $d_{l}$ and $l_{h}$ are quite dependent on the encoding technique used (Freitas, 2004). Nevertheless, Quinlan and Rivest (1989) propose selecting the attribute that minimizes $d_{l}+l_{h}$ at each node, and then pruning back the tree whenever replacing an internal node by a leaf decreases $d_{l}+l_{h}$.

A criterion derived from classical statistics is the multiple hypergeometric distribution $\left(P_{0}\right)$ (Agresti, 2002; Martin, 1997), which is an extension of Fischer's exact test for two binary variables. It can be regarded as the probability of obtaining the observed data given that the null hypothesis (of variable independence) is true. $P_{0}$ is given by:

$$
P_{0}\left(a_{i}, \mathbf{X}, y\right)=\left(\frac{\prod_{l=1}^{k} N_{\bullet}, y_{l} !}{N_{x} !}\right) \prod_{j=1}^{\left|a_{i}\right|}\left(\frac{N_{v_{j}, \bullet} !}{\prod_{m=1}^{k} N_{v_{j} \cap y_{m}} !}\right)
$$

The lower the values of $P_{0}$, the lower the probability of accepting the null hypothesis. Hence, the attribute that presents the lowest value of $P_{0}$ is chosen for splitting the current node in a decision tree.

Chandra and Varghese (2009) propose a new splitting criterion for partitioning nodes in decision trees. The proposed measure is designed to reduce the number of distinct classes resulting in each sub-tree after a split. Since the authors do not name their proposed measure, we call it CV (from Chandra-Varseghe) from now on. It is given by:

$$
C V\left(a_{i}, \mathbf{X}, y\right)=\sum_{j=1}^{\left|a_{i}\right|}\left[p_{v_{j}}, \times \frac{D_{v_{j}}}{D_{x}}\left(\sum_{l=1}^{D_{v_{j}}} p_{v_{j} \mid y_{l}}\right)\right]
$$

where $D_{x}$ counts the number of distinct class values among the set of instances in $\mathbf{X}$, and $D_{v_{j}}$ the number of distinct class values in partition $v_{j}$. The $\mathrm{CV}$ criterion must be minimized. The authors prove that $\mathrm{CV}$ is strictly convex (i.e., it achieves its minimum value at a boundary point) and cumulative (and thus, well-behaved). The authors argue that the experiments, which were performed on 19 data sets from the UCI repository (Frank and Asuncion, 2010), indicate that the proposed measure results in decision trees that are more compact (in terms of tree height), without compromising on accuracy when compared to the gain ratio and Gini index.

Chandra et al. (2010) propose the use of a distinct class based splitting measure (DCSM). It is given by: 


$$
\begin{gathered}
\operatorname{DCSM}\left(a_{i}, \mathbf{X}, y\right)=\sum_{j=1}^{\left|a_{i}\right|}\left[p_{v_{j},}, D_{v_{j}} \exp \left(D_{v_{j}}\right)\right. \\
\left.\times \sum_{l=1}^{k}\left[p_{y_{l} \mid v_{j}} \exp \left(\frac{D_{v_{j}}}{D_{x}}\left(1-\left(p_{y_{l} \mid v_{j}}\right)^{2}\right)\right)\right]\right]
\end{gathered}
$$

Note that the term $D_{v_{j}} \exp D_{v_{j}}$ deals with the number of distinct classes in a given partition $v_{j}$. As the number of distinct classes in a given partition increases, this term also increases. It means that purer partitions are preferred, and they are weighted according to the proportion of training instances that lie in the given partition. Also, note that $\frac{D_{v_{j}}}{D_{x}}$ decreases when the number of distinct classes decreases while $\left(1-\left(p_{y_{l} \mid v_{j}}\right)^{2}\right)$ decreases when there are more instances of a class compared to the total number of instances in a partition. These terms also favor partitions with a small number of distinct classes.

It can be noticed that the value of DCSM increases exponentially as the number of distinct classes in the partition increases, invalidating such splits. Chandra et al. (2010) argue that "this makes the measure more sensitive to the impurities present in the partition as compared to the existing measures." The authors demonstrate that DCSM satisfies two properties: convexity and well-behavedness. Finally, through empirical data, the authors affirm that DCSM provides more compact and more accurate trees than those provided by measures such as gain ratio and Gini index.

Many other split criteria for classification can be found in the literature, including relevance (Baim, 1988), misclassification error with confidence intervals (Kalkanis, 1993), RELIEF split criterion (Kononenko, 1994), QUEST split criterion (Loh and Shih, 1997), just to name a few.

\section{Regression Criteria}

All criteria presented so far are dedicated to classification problems. For regression problems, where the target variable $y$ is continuous, a common approach is to calculate the mean squared error (MSE) as a splitting criterion:

$$
\operatorname{MSE}\left(a_{i}, \mathbf{X}, y\right)=N_{x}^{-1} \sum_{j=1}^{\left|a_{i}\right|} \sum_{x_{l} \in v_{j}}\left(y\left(x_{l}\right)-\overline{y_{v_{j}}}\right)^{2}
$$

where $\overline{y_{v_{j}}}=N_{v_{j}, \bullet}^{-1} \sum_{x_{l} \in v_{j}} y\left(x_{l}\right)$. Just as with clustering, we are trying to minimize the within-partition variance. Usually, the sum of squared errors is weighted over each partition according to the estimated probability of an instance belonging to the given partition (Breiman et al., 1984). Thus, we should rewrite MSE to: 


$$
w M S E\left(a_{i}, \mathbf{X}, y\right)=\sum_{j=1}^{\left|a_{i}\right|} p_{v_{j}, \bullet} \sum_{x_{l} \in v_{j}}\left(y\left(x_{l}\right)-\overline{y_{v_{j}}}\right)^{2}
$$

Another common criterion for regression is the sum of absolute deviations (SAD) (Breiman et al., 1984), or similarly its weighted version given by:

$$
w S A D\left(a_{i}, \mathbf{X}, y\right)=\sum_{j=1}^{\left|a_{i}\right|} p_{v_{j}, \bullet} \sum_{x_{l} \in v_{j}} \operatorname{abs}\left(y\left(x_{l}\right)-\operatorname{median}\left(y_{v_{j}}\right)\right)
$$

where median $\left(y_{v_{j}}\right)$ is the target attribute's median of instances belonging to $\mathbf{X}_{\mathbf{a}_{\mathbf{i}}=\mathbf{v}_{\mathbf{j}}}$.

Quinlan (1992) proposes the use of the standard deviation reduction (SDR) for his pioneering system of model trees induction, M5. Wang and Witten (1997) extend the work of Quinlan in their proposed system M5', also employing the SDR criterion. It is given by:

$$
\operatorname{SDR}\left(a_{i}, \mathbf{X}, y\right)=\sigma_{X}-\sum_{j=1}^{\left|a_{i}\right|} p_{v_{j}, \bullet} \sigma_{v_{j}}
$$

where $\sigma_{X}$ is the standard deviation of instances in $\mathbf{X}$ and $\sigma_{v_{j}}$ the standard deviation of instances in $\mathbf{X}_{\mathrm{a}_{\mathrm{i}}=\mathbf{v}_{\mathrm{j}}}$. SDR should be maximized, i.e., the weighted sum of standard deviations of each partition should be as small as possible. Thus, partitioning the instance space according to a particular attribute $a_{i}$ should provide partitions whose target attribute variance is small (once again we are interested in minimizing the within-partition variance). Observe that minimizing the second term in SDR is equivalent to minimizing wMSE, but in SDR we are using the partition standard deviation $(\sigma)$ as a similarity criterion whereas in wMSE we are using the partition variance $\left(\sigma^{2}\right)$.

Buja and Lee (2001) propose two alternative regression criteria for binary trees: one-sided purity (OSP) and one-sided extremes (OSE). OSP is defined as:

$$
\operatorname{OSP}\left(a_{i}, d_{1}, d_{2}, \mathbf{X}, y\right)=\min _{d_{1}, d_{2}}\left(\sigma_{d_{1}}^{2}, \sigma_{d_{2}}^{2}\right)
$$

where $\sigma_{d_{i}}^{2}$ is the variance of partition $d_{i}$. The authors argue that by minimizing this criterion for all possible splits, we find a split whose partition (either $d_{1}$ or $d_{2}$ ) presents the smallest variance. Typically, this partition is less likely to be split again. Buja and Lee (2001) also propose the OSE criterion: 


$$
\begin{array}{r}
O S E\left(a_{i}, d_{1}, d_{2}, \mathbf{X}, y\right)=\min _{d_{1}, d_{2}}\left(\overline{y_{d_{1}}}, \bar{y}_{d_{2}}\right) \\
\text { or, conservely: } \\
O S E\left(a_{i}, d_{1}, d_{2}, \mathbf{X}, y\right)=\max _{d_{1}, d_{2}}\left(\overline{y_{d_{1}}}, \bar{y}_{d_{2}}\right)
\end{array}
$$

The authors argue that whereas the mean values have not been thought of as splitting criteria, "in real data, the dependence of the mean response on the predictor variables is often monotone; hence extreme response values are often found on the periphery of variable ranges (...), the kind of situations to each the OSE criteria would respond".

Alpaydin (2010) mentions the use of the worst possible error (WPE) as a valid criterion for splitting nodes:

$$
W P E\left(a_{i}, \mathbf{X}, y\right)=\max _{j} \max _{l}\left[a b s\left(y\left(x_{l}\right)-\overline{y_{v_{j}}}\right)\right]
$$

Alpaydin (2010) states that by using WPE we can guarantee that the error for any instance is never larger than a given threshold $\Delta$. This analysis is useful because the threshold $\Delta$ can be seen as a complexity parameter that defines the fitting level provided by the tree, given that we use it for deciding when interrupting its growth. Larger values of $\Delta$ lead to smaller trees that could underfit the data whereas smaller values of $\Delta$ lead to larger trees that risk overfitting. A deeper appreciation of underfitting, overfitting and tree complexity is presented later, when pruning is discussed.

Other regression criteria that can be found in the literature are MPI for regression (Taylor and Jones, 1996), Lee's criteria (Lee, 2001), GUIDE's criterion (Loh, 2002), and SMOTI's criterion (Malerba et al., 2004), just to name a few. Tables 2.1 and 2.2 show all univariate splitting criteria cited in this section, as well as their corresponding references, listed in chronological order.

\section{Multivariate Splits}

All criteria presented so far are intended for building univariate splits. Decision trees with multivariate splits (known as oblique, linear or multivariate decision trees) are not so popular as the univariate ones, mainly because they are harder to interpret. Nevertheless, researchers reckon that multivariate splits can improve the performance of the tree in several data sets, while generating smaller trees (Heath et al., 1993; Murthy et al., 1994; Rokach and Maimon, 2005). Clearly, there is a tradeoff to consider in allowing multivariate tests: simple tests may result in large trees that are hard to understand, yet multivariate tests may result in small trees with tests hard to understand (Utgoff and Brodley, 1990). Figure 2.2 presents a univariate decision tree that needs 5 splits to separate two classes, which could be easily separated by a single oblique split.

A decision tree with multivariate splits is able to produce polygonal (polyhedral) partitions of the attribute space (hyperplanes at an oblique orientation to the attribute axes) whereas univariate 
Table 2.1: Univariate splitting criteria for classification.

\begin{tabular}{|c|c|c|}
\hline Category & Criterion & References \\
\hline Info Theory & $\begin{array}{l}\text { GMI } \\
\text { Information Gain } \\
\text { G Statistic } \\
\text { Gain Ratio } \\
\text { CAIR } \\
\text { Normalized Gain } \\
\text { Average Gain } \\
\text { MEE }\end{array}$ & $\begin{array}{l}\text { (Gleser and Collen, 1972; Sethi and Sarvarayudu, 1982) } \\
\text { (Ching et al., 1995; Talmon, 1986) } \\
\text { (Casey and Nagy, 1984; Hartmann et al., 1982; Varshney et al., 1982) } \\
\text { (Quinlan, 1986) } \\
\text { (Mingers, 1987, 1989b) } \\
\text { (Quinlan, 1986, 1993) } \\
\text { (Ching et al., 1995) } \\
\text { (Jun et al., 1997) } \\
\text { (Wang and Jiang, 2007) } \\
\text { (Sá et al., 2009) }\end{array}$ \\
\hline Distance-based & $\begin{array}{l}\text { KS Distance } \\
\text { Gini Index } \\
\text { Twoing } \\
\chi^{2} \\
\text { Distance } \\
\text { Multi-class KS } \\
\text { ORT } \\
\text { MPI } \\
\tau \text { Index }\end{array}$ & $\begin{array}{l}\text { (Friedman, 1977; Rounds, 1980) } \\
\text { (Breiman et al., 1984) } \\
\text { (Breiman et al., 1984) } \\
\text { (Mingers, 1989b; White and Liu, 1994; Zhou and Dillon, 1991) } \\
\text { (De Mántaras, 1991) } \\
\text { (Haskell and Noui-Mehidi, 1991; Utgoff and Clouse, 1996) } \\
\text { (Fayyad and Irani, 1992) } \\
\text { (Taylor and Silverman, 1993) } \\
\text { (Mola and Siciliano, 1997) }\end{array}$ \\
\hline Other & $\begin{array}{l}\text { Permutation } \\
\text { Relevance } \\
\text { MDL criterion } \\
\text { Mis. Error with CI } \\
\text { RELIEF } \\
\text { QUEST criterion } \\
P_{0} \\
\text { CV } \\
\text { DCSM }\end{array}$ & $\begin{array}{l}\text { (Li and Dubes, 1986) } \\
\text { (Baim, 1988) } \\
\text { (Quinlan and Rivest, 1989) } \\
\text { (Kalkanis, 1993) } \\
\text { (Kononenko, 1994) } \\
\text { (Loh and Shih, 1997) } \\
\text { (Agresti, 2002; Martin, 1997) } \\
\text { (Chandra and Varghese, 2009) } \\
\text { (Chandra et al., 2010) }\end{array}$ \\
\hline
\end{tabular}

Table 2.2: Univariate splitting criteria for regression.

\begin{tabular}{l|l}
\hline Criterion & References \\
\hline (w)MSE & (Breiman et al., 1984) \\
(w)SAD & (Breiman et al., 1984) \\
SDR & (Quinlan, 1992; Wang and Witten, 1997) \\
MPI-R & (Taylor and Jones, 1996) \\
OSP & (Buja and Lee, 2001) \\
OSE & (Buja and Lee, 2001) \\
Lee's & (Lee, 2001) \\
GUIDE's & (Loh, 2002) \\
SMOTI's & (Malerba et al., 2004) \\
WPE & (Alpaydin, 2010) \\
\hline
\end{tabular}

trees can only produce hyper-rectangles parallel to the attribute axes. The tests at each node have the form:

$$
w_{0}+\sum_{i=1}^{n} w_{i} a_{i}(x) \leq 0
$$

where $w_{i}$ is a real-valued coefficient associated to the $i^{\text {th }}$ attribute and $w_{0}$ the disturbance coefficient of the test.

CART (Classification and Regression Trees) (Breiman et al., 1984) is one of the first systems that allowed multivariate splits. It employs a hill-climbing strategy with a backward attribute elimination for finding good (albeit suboptimal) linear combinations of attributes in non-terminal nodes. It is a fully-deterministic algorithm with no built-in mechanisms to escape local-optima. Breiman et al. (1984) point out that the proposed algorithm has much room for improvement. 

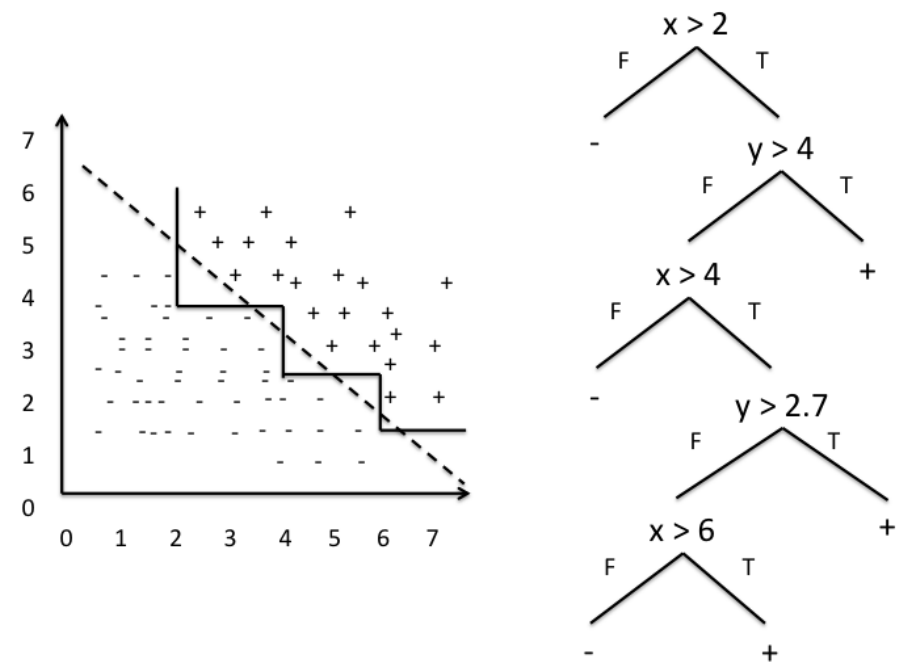

Figure 2.2: A bivariate input space in a binary-class problem and its corresponding univariate decision tree. The single oblique split that separates the two classes is $x+y \leq 8$. Adapted from (Brodley and Utgoff, 1995).

Another approach for building oblique decision trees is LMDT (Linear Machine Decision Trees) (Brodley and Utgoff, 1992; Utgoff and Brodley, 1991), which is an evolution of the perceptron tree method (Utgoff, 1989). Each non-terminal node holds a linear machine (Nilsson, 1990), which is a set of $k$ linear discriminant functions that are used collectively to assign an instance to one of the $k$ existing classes. LMDT uses heuristics to determine when a linear machine has stabilized (since convergence cannot be guaranteed). More specifically, for handling non-linearly separable problems, a method similar to simulated annealing (SA) is used (called thermal training). Draper and Brodley (1994) show how LMDT can be altered to induce decision trees that minimize arbitrary misclassification cost functions.

SADT (Simulated Annealing of Decision Trees) (Heath et al., 1993) is a system that employs SA for finding good coefficient values for attributes in non-terminal nodes of decision trees. First, it places a hyperplane in a canonical location, and then iteratively perturbs the coefficients in small random amounts. At the beginning, when the temperature parameter of the SA is high, practically any perturbation of the coefficients is accepted regardless of the goodness-of-split value (the value of the utilised splitting criterion). As the SA cools down, only perturbations that improve the goodness-of-split are likely to be allowed. Although SADT can eventually escape from local-optima, its efficiency is compromised since it may consider tens of thousands of hyperplanes in a single node during annealing.

OC1 (Oblique Classifier 1) (Murthy et al., 1993, 1994) is yet another oblique decision tree system. It is a thorough extension of CART's oblique decision tree strategy. OC1 presents the advantage of being more efficient than the previously described systems. For instance, in the worst case scenario, OC1's running time is $O\left(\log _{n}\right)$ times greater than the worst case scenario of univariate decision trees, i.e., $O\left(n N^{2} \log _{N}\right)$ versus $O\left(n N^{2}\right)$. OC1 searches for the best univariate 
split as well as the best oblique split, and it only employs the oblique split when it improves over the univariate split ${ }^{3}$. It uses both a deterministic heuristic search (as employed in CART) for finding local-optima and a non-deterministic search (as employed in SADT - though not SA) for escaping local-optima.

During the deterministic search, OC1 perturbs the hyperplane coefficients sequentially (much in the same way CART does) until no significant gain is achieved according to an impurity measure. More specifically, consider hyperplane $H=w_{0}+\sum_{i=1}^{n} w_{i} a_{i}(x)=0$, and that we substitute an instance $x_{j}$ in $H$, i.e., $H=w_{0}+\sum_{i=1}^{n} w_{i} a_{i}\left(x_{j}\right)=Z_{j}$. The sign of $Z_{j}$ indicates whether an instance $x_{j}$ is above or below the hyperplane $H$. If $H$ splits $\mathbf{X}$ perfectly, then all instances belonging to the same class will have the same sign of $Z$. For finding the local-optimal set of coefficients, OC1 employs a sequential procedure that works as follows: treat coefficient $w_{i}$ as a variable and all other coefficients as constants. The condition that instance $x_{j}$ is above hyperplane $H$ can be written as:

$$
w_{i}>\left[\frac{w_{i} a_{i}\left(x_{j}\right)-Z_{j}}{a_{i}\left(x_{j}\right)} \equiv U_{j}\right]
$$

assuming $a_{i}\left(x_{j}\right)>0$, which is ensured through normalization. With the definition in (2.32), an instance is above the hyperplane if $w_{i}>U_{j}$ and below otherwise. By plugging each instance $x \in$ $\mathrm{X}$ in (2.32), we obtain $N_{x}$ constraints on the value of $w_{i}$. Hence, the problem is reduced on finding the value of $w_{i}$ that satisfies the greatest possible number of constraints. This problem is easy to solve optimally: simply sort all the values $U_{j}$, and consider setting $w_{i}$ to the midpoint between each pair of different class values. For each distinct placement of the coefficient $w_{i}$, OC1 computes the impurity of the resulting split, and replaces original coefficient $w_{i}$ by the recently discovered value if there is reduction on impurity. The pseudocode of this deterministic perturbation method is presented in Algorithm 2.

The parameter $P_{\text {stag }}$ (stagnation probability) is the probability that a hyperplane is perturbed to a location that does not change the impurity measure. To prevent the stagnation of impurity, $P_{\text {stag }}$ decreases by a constant amount each time OC1 makes a "stagnant" perturbation, which means only a constant number of such perturbations will occur at each node. $P_{\text {stag }}$ is reset to 1 every time the global impurity measure is improved. It is a user-defined parameter.

After a local-optimal hyperplane $H$ is found, it is further perturbed by a randomized vector, as follows: it computes the optimal amount by which $H$ should be perturbed along the random direction dictated by a random vector. To be more precise, when a hyperplane $H=w_{0}+$ $\sum_{i=1}^{n} w_{i} a_{i}(x)$ cannot be improved by deterministic perturbation (Algorithm 2), OC1 repeats the following loop $J$ times (where $J$ is a user-specified parameter, set to 5 by default):

\footnotetext{
${ }^{3} \mathrm{OC} 1$ only allows the option of employing oblique splits when $N>2 n$, though this threshold can be user-defined.
} 


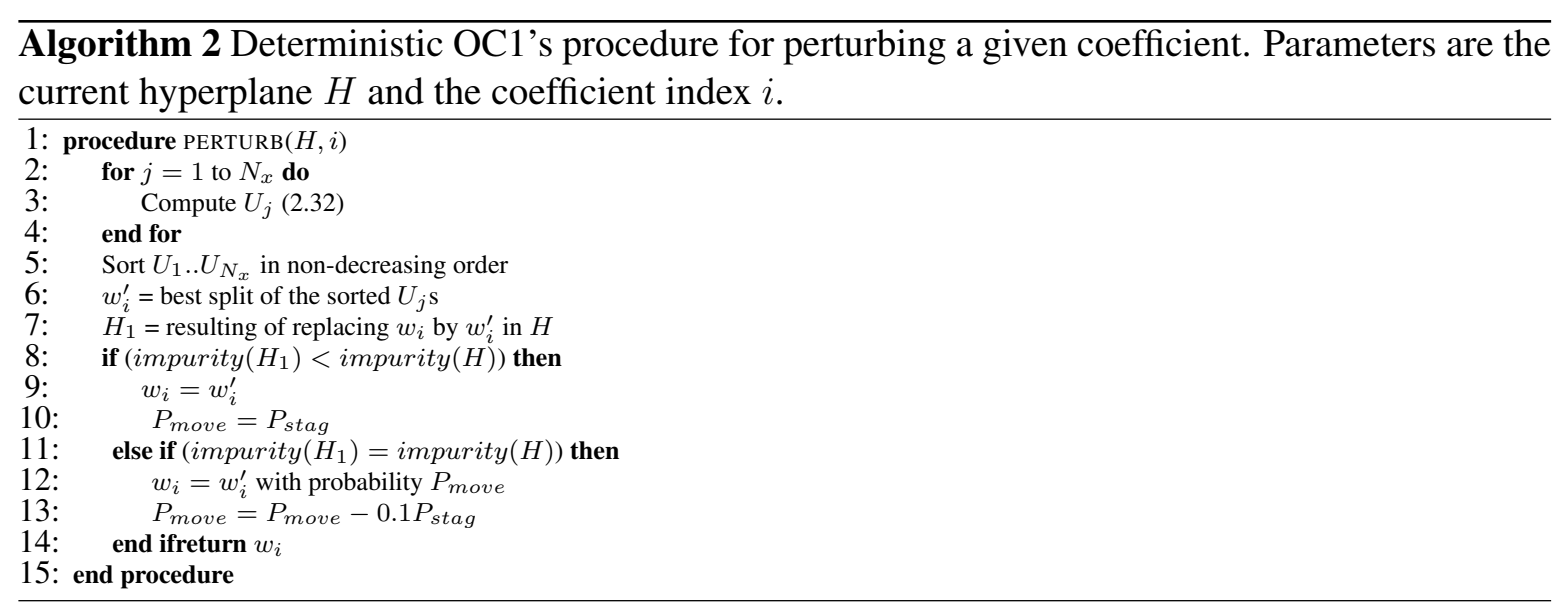

- Choose a random vector $R=\left[r_{0}, r_{1}, \ldots, r_{n}\right]$;

- Let $\alpha$ be the amount by which we want to perturb $H$ in the direction $R$. More specifically, let $H_{1}=\left(w_{0}+\alpha r_{0}\right)+\sum_{i=1}^{n}\left(w_{i}+\alpha r_{i}\right) a_{i}(x)$;

- Find the optimal value for $\alpha$;

- If the hyperplane $H_{1}$ decreases the overall impurity, replace $H$ with $H 1$, exit this loop and begin the deterministic perturbation algorithm for the individual coefficients.

Note that we can treat $\alpha$ as the only variable in the equation for $H_{1}$. Therefore each of the $N$ examples, if plugged into the equation for $H_{1}$, imposes a constraint on the value of $\alpha$. OC1 can use its own deterministic coefficient perturbation method (Algorithm 2) to compute the best value of $\alpha$. If $J$ random jumps fail to improve the impurity measure, $\mathrm{OC} 1$ halts and uses $H$ as the split for the current tree node. Regarding the impurity measure, $\mathrm{OC} 1$ allows the user to choose among a set of splitting criteria, such as information gain, Gini index, twoing criterion, among others.

Ittner (1996) proposes using OC1 over an augmented attribute space, generating non-linear decision trees. The key idea involved is to "build" new attributes by considering all possible pairwise products and squares of the original set of $n$ attributes. As a result, a new attribute space with $\left(n^{2}+3 n\right) / 2$ is formed, i.e., the sum of $n$ original attributes, $n$ squared ones and $(n(n-1)) / 2$ pairwise products of the original attributes. To illustrate, consider a binary attribute space $\left\{a_{1}, a_{2}\right\}$. The augmented attribute space would contain 5 attributes, i.e., $b_{1}=a_{1}, b_{2}=a_{2}, b_{3}=a_{1} a_{2}, b_{4}=$ $a_{1}^{2}, b_{5}=a_{2}^{2}$. Figure 2.3 shows an interesting example of a binary class two-dimensional data set called spiral data set, and the corresponding axis-parallel, oblique and non-linear partitioning of the attribute space.

A similar approach of transforming the original attributes is taken in (Liu and Setiono, 1998), in which the authors propose the BMDT system. In BMDT, a 2-layer feedforward neural network is employed to transform the original attribute space in a space in which the new attributes are linear combinations of the original ones. This transformation is performed through a hyperbolic tangent function at the hidden units. After transforming the attributes, a univariate decision-tree 


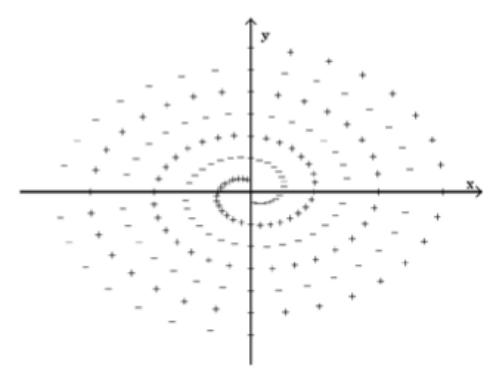

(a)

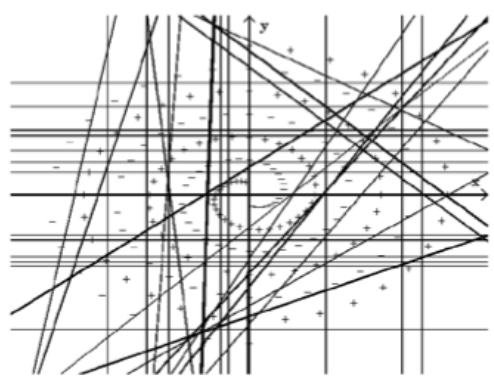

(c)

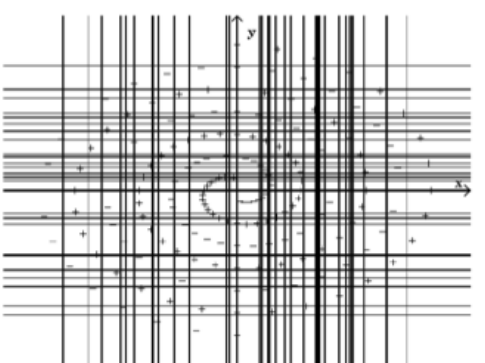

(b)

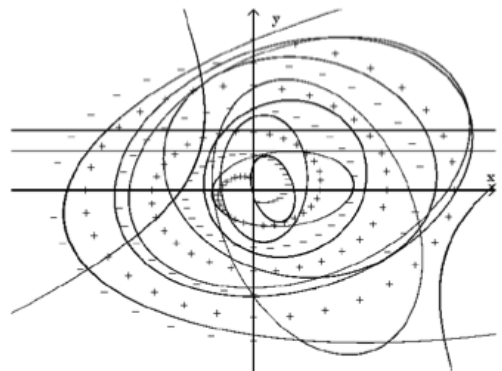

(d)

Figure 2.3: (a) A bivariate input space in a binary-class problem: the spiral data set. (b) The corresponding axis-parallel partitioning. (c) The corresponding oblique partitioning. (d) The corresponding non-linear partitioning. Adapted from (Ittner, 1996).

induction algorithm is employed over this new attribute space. Finally, a procedure replaces the transformed attributes by the original ones, which means that the univariate tests in the recently built decision tree become multivariate tests, and thus the univariate tree becomes an oblique tree.

Shah and Sastry (1999) propose the APDT (Alopex Perceptron Decision Tree) system. It is an oblique decision tree inducer that makes use of a new splitting criterion, based on the level of non-separability of the input instances. They argue that because oblique decision trees can realize arbitrary piecewise linear separating surfaces, it seems better to base the evaluation function on the degree of separability of the partitions rather than on the degree of purity of them. APDT runs the Perceptron algorithm for estimating the number of non-separable instances belonging to each one of the binary partitions provided by an initial hyperplane. Then, a correlation-based optimization algorithm called Alopex (Unnikrishnan and Venugopal, 1994) is employed for tuning the hyperplane weights taking into account the need of minimizing the new split criterion based on the degree of separability of partitions. Shah and Sastry (1999) also propose a pruning algorithm based on genetic algorithms (further presented in Chapter 4 of this thesis).

Several other oblique decision-tree systems were proposed employing different strategies for defining the weights of hyperplanes and for evaluating the generated split. Some examples include: the system proposed by Bobrowski and Kretowski (2000), which employs heuristic sequential search (combination of sequential backward elimination and sequential forward selection) for defining hyperplanes and a dipolar criterion for evaluating splits; the omnivariate decision tree inducer proposed by Yildiz and Alpaydin (2001), where the non-terminal nodes may be univariate, 
linear, or nonlinear depending on the outcome of comparative statistical tests on accuracy, allowing the split to match automatically the complexity of the node according to the subproblem defined by the data reaching that node; Li et al. (2003) propose using tabu search and a variation of linear discriminant analysis for generating multivariate splits, arguing that their algorithm runs faster than most oblique tree inducers, since its computing time increases linearly with the number of instances; Tan and Dowe (2004) proposes inducing oblique trees through a MDL-based splitting criterion and the evolution strategy as a meta-heuristic to search for the optimal hyperplane within a node. For regression oblique trees please refer to (De'ath, 2002; Hsiao and Shih, 2007; Larsen and Speckman, 2004).

For the interested reader, it is worth mentioning that there are methods that induce oblique decision trees with optimal hyperplanes, discovered through linear programming (Bennett and Mangasarian, 1992, 1994; Mangasarian et al., 1990). Though these methods can find the optimal hyperplanes for specific splitting measures, the size of the linear program grows very fast with the number of instances and attributes.

For a discussion on several papers that employ evolutionary algorithms for induction of oblique decision trees (to evolve either the hyperplanes or the whole tree), the reader is referred to Chapter 4. Table 2.3 presents a summary of some systems proposed for induction of oblique decision trees.

Table 2.3: Multivariate splits.

\begin{tabular}{l|l|l}
\hline System & Criterion & Hyperplane Strategy \\
\hline CART (Breiman et al., 1984) & Gini Index/Twoing & Hill-Climbing with SBE \\
LMDT (Brodley and Utgoff, 1992) & Misclassification Error & Linear Machine with Thermal Training \\
SADT (Heath et al., 1993) & Sum-Minority & Simulated Annealing \\
OC1 (Murthy et al., 1993, 1994) & Info gain, Gini Index, Twoing, etc. & Hill-Climbing with Randomization \\
BMDT (Liu and Setiono, 1998) & 2-Layer Feedforward Neural Network \\
APDT (Shah and Sastry, 1999) & Gain Ratio & Alopex \\
Bobrowski and Kretowski (2000) & Separability a & Heuristic Sequential Search \\
Omni (Yildiz and Alpaydin, 2001) & Dipolar criterion & MLP Neural Network \\
LDTS (Li et al., 2003) & Misclassification Error & LDA with Tabu Search \\
MML (Li et al., 2003) & Info Gain & Evolution Strategy \\
Geometric DT (Manwani and Sastry, 2009) & MDL-based & Gini-Index \\
\hline a The authors of the criterion do not explicitly name it, so we call it "Separability", since the criterion is based on the degree of \\
linear separability.
\end{tabular}

\subsubsection{Stopping Criteria}

The top-down induction of a decision tree is recursive and it continues until a stopping criterion (or some stopping criteria) is satisfied. Some popular stopping criteria are (Esposito et al., 1997; Rokach and Maimon, 2005):

1. Reaching class homogeneity: when all instances that reach a given node belong to the same class, there is no reason to split this node any further;

2. Reaching attribute homogeneity: when all instances that reach a given node have the same attribute values (though not necessarily the same class value); 
3. Reaching the maximum tree depth: a parameter tree depth can be specified to avoid deep trees;

4. Reaching the minimum number of instances for a non-terminal node: a parameter minimum number of instances for a non-terminal node can be specified to avoid (or at least alleviate) the data fragmentation problem;

5. Failing to exceed a threshold when calculating the splitting criterion: a parameter splitting criterion threshold can be specified for avoiding weak splits.

Criterion 1 is universally accepted and it is implemented in most top-down decision-tree induction algorithms to date. Criterion 2 deals with the case of contradictory instances, i.e., identical instances regarding $A$, but with different class values. Criterion 3 is usually a constraint regarding tree complexity, specially for those cases in which comprehensibility is an important requirement, though it may affect complex classification problems which require deeper trees. Criterion 4 implies that small disjuncts (i.e., tree leaves covering a small number of objects) can be ignored since they are error-prone. Note that eliminating small disjuncts can be harmful to exceptions - particularly in a scenario of imbalanced classes. Criterion 5 is heavily dependent on the splitting measure used. An example presented in (Esposito et al., 1997) clearly indicates a scenario in which using criterion 5 prevents the growth of a 100\% accurate decision tree (a problem usually referred to as the horizon effect (Breiman et al., 1984; Quinlan, 1993)).

The five criteria presented above can be seen as pre-pruning strategies, since they "prematurely" interrupt the growth of the tree. Note that most of the criteria discussed here may harm the growth of an accurate decision tree. Indeed there is practically a consensus in the literature that decision trees should be overgrown instead. For that, the stopping criterion used should be as loose as possible (e.g., until a single instance is contemplated by the node or until criterion 1 is satisfied). Then, a post-pruning technique should be employed in order to prevent data overfitting - a phenomenon that happens when the classifier over-learns the data, that is, when it learns all data peculiarities - including potential noise and spurious patterns - that are specific to the training set and do not generalise well to the test set. Post-pruning techniques are covered in the next section.

\subsubsection{Pruning}

This section reviews strategies of pruning, normally referred to as post-pruning techniques. Pruning is usually performed in decision trees for enhancing tree comprehensibility (by reducing its size) while maintaining (or even improving) accuracy. It was originally conceived as a strategy for tolerating noisy data, though it was found that it could improve decision tree accuracy in many noisy data sets (Breiman et al., 1984; Quinlan, 1986, 1987b).

A pruning method receives as input an unpruned tree $T$ and outputs a decision tree $T^{\prime}$ formed by removing one or more subtrees from $T$. It replaces non-terminal nodes by leaf nodes according 
to a given heuristic. Next, we present the six most well-known pruning methods for decision trees (Breslow and Aha, 1997; Esposito et al., 1997): 1) reduced-error pruning; 2) pessimistic error pruning; 3) minimum error pruning; 4) critical-value pruning; 5) cost-complexity pruning; and 6) error-based pruning.

\section{Reduced-error Pruning (REP)}

Reduced-error pruning is a conceptually simple strategy proposed by Quinlan (1987b). It uses a pruning set (a part of the training set) to evaluate the goodness of a given subtree from $T$. The idea is to evaluate each non-terminal node $t \in \zeta_{T}$ with regard to the classification error in the pruning set. If such an error decreases when we replace the subtree $T^{(t)}$ by a leaf node, than $T^{(t)}$ must be pruned.

Quinlan imposes a constraint: a node $t$ cannot be pruned if it contains a subtree that yields a lower classification error in the pruning set. The practical consequence of this constraint is that REP should be performed in a bottom-up fashion. The REP pruned tree $T^{\prime}$ presents an interesting optimality property: it is the smallest most accurate tree resulting from pruning original tree $T$ (Quinlan, 1987b). Besides this optimality property, another advantage of REP is its linear complexity, since each node is visited only once in $T$. An obvious disadvantage is the need of using a pruning set, which means one has to divide the original training set, resulting in less instances to grow the tree. This disadvantage is particularly serious for small data sets.

\section{Pessimistic error Pruning (PEP)}

Also proposed by Quinlan (1987b), the pessimistic error pruning uses the training set for both growing and pruning the tree. The apparent error rate, i.e., the error rate calculated over the training set, is optimistically biased and cannot be used to decide whether pruning should be performed or not. Quinlan thus proposes adjusting the apparent error according to the continuity correction for the binomial distribution $(c c)$ in order to provide a more realistic error rate. Consider the apparent error of a pruned node $t$, and the error of its entire subtree $T^{(t)}$ before pruning is performed, respectively:

$$
\begin{aligned}
r^{(t)} & =\frac{E^{(t)}}{N_{x}^{(t)}} \\
r^{T^{(t)}} & =\frac{\sum_{s \in \lambda_{T^{(t)}}} E^{(s)}}{\sum_{s \in \lambda_{T^{(t)}}} N_{x}^{(s)}} .
\end{aligned}
$$

Modifying (2.33) and (2.34) according to $c c$ results in: 


$$
\begin{gathered}
r_{c c}^{(t)}=\frac{E^{(t)}+1 / 2}{N_{x}^{(t)}} \\
r_{c c}^{T^{(t)}}=\frac{\sum_{s \in \lambda_{T^{(t)}}} E^{(s)}+1 / 2}{\sum_{s \in \lambda_{T^{(t)}}} N_{x}^{(s)}}=\frac{\frac{\left|\lambda_{T^{(t)}}\right|}{2} \sum_{s \in \lambda_{T^{(t)}}} E^{(s)}}{\sum_{s \in \lambda_{T^{(t)}}} N_{x}^{(s)}} .
\end{gathered}
$$

For the sake of simplicity, we will refer to the adjusted number of errors rather than the adjusted error rate, i.e., $E_{c c}^{(t)}=E^{(t)}+1 / 2$ and $E_{c c}^{T^{(t)}}=\left(\left|\lambda_{T^{(t)}}\right| / 2\right) \sum_{s \in \lambda_{T^{(t)}}} E^{(s)}$. Ideally, pruning should occur if $E_{c c}^{(t)} \leq E_{c c}^{T^{(t)}}$, but note that this condition seldom holds, since the decision tree is usually grown up to the homogeneity stopping criterion (criterion 1 in Section 2.3.2), and thus $E_{c c}^{T^{(t)}}=$ $\left|\lambda_{T^{(t)}}\right| / 2$ whereas $E_{c c}^{(t)}$ will very probably be a higher value. In fact, due to the homogeneity stopping criterion, $E_{c c}^{T^{(t)}}$ becomes simply a measure of complexity which associates each leaf node with a cost of $1 / 2$. Quinlan, aware of this situation, weakens the original condition

$$
E_{c c}^{(t)} \leq E_{c c}^{T^{(t)}}
$$

to

$$
E_{c c}^{(t)} \leq E_{c c}^{T^{(t)}}+S E\left(E_{c c}^{T^{(t)}}\right)
$$

where

$$
S E\left(E_{c c}^{T^{(t)}}\right)=\sqrt{\frac{E_{c c}^{T^{(t)}} *\left(N_{x}^{(t)}-E_{c c}^{T^{(t)}}\right)}{N_{x}^{(t)}}}
$$

is the standard error for the subtree $T^{(t)}$, computed as if the distribution of errors were binomial.

PEP is computed in a top-down fashion, and if a given node $t$ is pruned, its descendants are not examined, which makes this pruning strategy quite efficient in terms of computational effort. As a point of criticism, Esposito et al. (1997) point out that the introduction of the continuity correction in the estimation of the error rate has no theoretical justification, since it was never applied to correct over-optimistic estimates of error rates in statistics.

\section{Minimum error Pruning (MEP)}

Originally proposed by Niblett and Bratko (1986) and further extended by Cestnik and Bratko (1991), minimum error pruning is a bottom-up approach that seeks to minimize the expected error rate for unseen cases. It estimates the expected error rate in node $t\left(E E^{(t)}\right)$ as follows:

$$
E E^{(t)}=\min _{y_{l}}\left[\frac{N_{x}^{(t)}-N_{\bullet, y_{l}}^{(t)}+\left(1-p_{\bullet, y_{l}}^{(t)}\right) \times m}{N_{x}^{(t)}+m}\right]
$$


where $m$ is a parameter that determines the importance of the a priori probability on the estimation of the error. Eq. (2.39), presented in (Cestnik and Bratko, 1991), is a generalisation of the expected error rate presented in (Niblett and Bratko, 1986) if we assume that $m=k$ and that $p_{\bullet, y_{l}}^{(t)}=$ $1 / k, \forall y_{l} \in Y$.

MEP is performed by comparing $E E^{(t)}$ with the weighted sum of the expected error rate of all children nodes from $t$. Each weight is given by $p_{v_{j}, \bullet}$, assuming $v_{j}$ is the partition corresponding to the $j^{t h}$ child of $t$. A disadvantage of MEP is the need of setting the ad-hoc parameter $m$. Usually, the higher the value of $m$, the more severe the pruning. Cestnik and Bratko (1991) suggest that a domain expert should set $m$ according to the level of noise in the data. Alternatively, a set of trees pruned with different values of $m$ could be offered to the domain expert, so he/she can choose the best one according to his/her experience.

\section{Critical-value Pruning (CVP)}

The critical-value pruning, proposed by Mingers (1987), is quite similar to the pre-pruning strategy criterion 5 in Section 2.3.2. It is a bottom-up procedure that prunes a given non-terminal node $t$ in case the value of its splitting measure is below a pre-determined threshold $c v$. Mingers (1987) proposes the following two-step procedure for performing CVP:

1. Prune $T$ for increasing values of $c v$, generating a set of pruned trees;

2. Choose the best tree among the set of trees (that includes $T$ ) by measuring each tree's accuracy (based on a pruning set) and significance (through the previously presented $\mathrm{G}$ statistic).

The disadvantage of CVP is the same of REP - the need of a pruning set. In addition, CVP does not present the optimality property that REP does, so there is no guarantee that the best tree found in step 2 is the smallest optimally pruned subtree of $T$, since the pruning step was performed based on the training set.

\section{Cost-complexity Pruning (CCP)}

Cost-complexity pruning is the post-pruning strategy of the CART system, detailed in (Breiman et al., 1984). It consists of two steps:

1. Generate a sequence of increasingly smaller trees, beginning with $T$ and ending with the root node of $T$, by successively pruning the subtree yielding the lowest cost complexity, in a bottom-up fashion;

2. Choose the best tree among the sequence based on its relative size and accuracy (either on a pruning set, or provided by a cross-validation procedure in the training set). 
The idea within step 1 is that pruned tree $T_{i+1}$ is obtained by pruning the subtrees that show the lowest increase in the apparent error (error in the training set) per pruned leaf. Since the apparent error of pruned node $t$ increases by the amount $r^{(t)}-r^{T^{(t)}}$, whereas its number of leaves decreases by $\left|\lambda_{T^{(t)}}\right|-1$ units, the following ratio measures the increase in apparent error rate per pruned leaf:

$$
\alpha=\frac{r^{(t)}-r^{T^{(t)}}}{\left|\lambda_{T^{(t)}}\right|-1}
$$

Therefore, $T_{i+1}$ is obtained by pruning all nodes in $T_{i}$ with the lowest value of $\alpha . T_{0}$ is obtained by pruning all nodes in $T$ whose $\alpha$ value is 0 . It is possible to show that each tree $T_{i}$ is associated to a distinct value $\alpha_{i}$, such that $\alpha_{i}<\alpha_{i+1}$. Building the sequence of trees in step 1 takes quadratic time with respect to the number of internal nodes.

Regarding step 2, CCP chooses the smallest tree whose error (either on the pruning set or on cross-validation) is not more than one standard error (SE) greater than the lowest error observed in the sequence of trees. This strategy is known as "1-SE" variant since the work of Esposito et al. (1995a), which proposes ignoring the standard error constraint, calling the strategy of selecting trees based only on accuracy of "0-SE". It is argued that 1-SE has a tendency of overpruning trees, since its selection is based on a conservative constraint (Esposito et al., 1995a, 1997).

\section{Error-based Pruning (EBP)}

This strategy was proposed by Quinlan and it is implemented as the default pruning strategy of C4.5 (Quinlan, 1993). It is an improvement over PEP, based on a far more pessimistic estimate of the expected error. Unlike PEP, EBP performs a bottom-up search, and it performs not only the replacement of non-terminal nodes by leaves but also the grafting ${ }^{4}$ of subtree $T^{(t)}$ onto the place of parent $t$. Grafting is exemplified in Figure 2.4.

Since grafting is potentially a time-consuming task, only the child subtree $T^{\left(t^{\prime}\right)}$ of $t$ with the greatest number of instances is considered to be grafted onto the place of $t$.

For deciding whether to replace a non-terminal node by a leaf (subtree replacement), to graft a subtree onto the place of its parent (subtree raising) or not to prune at all, a pessimistic estimate of the expected error is calculated by using an upper confidence bound. Assuming that errors in the training set are binomially distributed with a given probability $p$ in $N_{x}^{(t)}$ trials, it is possible to compute the exact value of the upper confidence bound as the value of $p$ for which a binomially distributed random variable $P$ shows $E^{(t)}$ successes in $N_{x}^{(t)}$ trials with probability $C F$. In other words, given a particular confidence $C F$ (C4.5 default value is $C F=25 \%$ ), we can find the upper bound of the expected error $\left(E E_{U B}\right)$ as follows:

\footnotetext{
${ }^{4}$ Grafting is a term introduced by Esposito et al. (1995b). It is also known as subtree raising (Witten and Frank, 1999).
} 

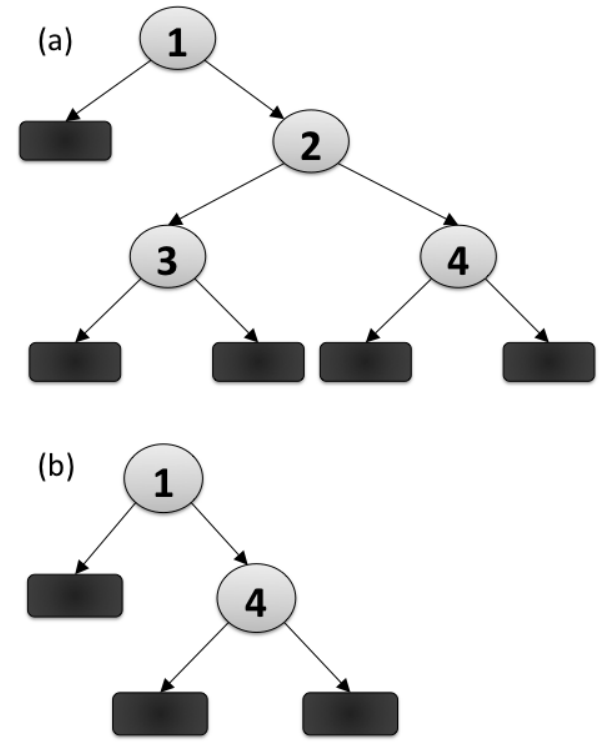

Figure 2.4: Grafting of subtree rooted in 4 onto the place of parent 2 . In (a) the original tree $T$ and in (b) the pruned tree $T^{\prime}$.

$$
E E_{U B}=\frac{f+\frac{z^{2}}{2 N_{x}}+z \sqrt{\frac{f}{N_{x}}-\frac{f^{2}}{N_{x}}+\frac{z^{2}}{4 N_{x}^{2}}}}{1+\frac{z^{2}}{N_{x}}}
$$

where $f=E^{(t)} / N_{x}$ and $z$ is the number of standard deviations corresponding to the confidence $C F$ (e.g., for $C F=25 \%, z=0.69$ ).

In order to calculate the expected error of node $t\left(E E^{(t)}\right)$, one must simply compute $N_{x}^{(t)} \times$ $E E_{U B}$. For evaluating a subtree $T^{(t)}$, one must sum the expected error of every leaf of that subtree, i.e, $\sum_{s \in \lambda_{T^{(t)}}} E E^{(s)}$. Hence, given a non-terminal node $t$, it is possible to decide whether one should perform subtree replacement (when condition $E E^{(t)} \leq E E^{T^{(t)}}$ holds), subtree raising (when conditions $\exists j \in \zeta_{t}, E E^{(j)}<E E^{(t)} \wedge \forall i \in \zeta_{t}, N_{x}^{(i)}<N_{x}^{(j)}$ hold), or not to prune $t$ otherwise.

An advantage of EBP is the new grafting operation that allows pruning useless branches without ignoring interesting lower branches (an elegant solution to the horizon effect problem). A drawback of the method is the parameter $C F$, even though it represents a confidence level. Smaller values of $C F$ result in more pruning.

\section{Empirical Evaluations}

Some studies in the literature performed empirical analyses for evaluating pruning strategies. For instance, Quinlan (1987b) compared four methods of tree pruning (three of them presented in the previous sections - REP, PEP and CCP 1-SE). He argued that those methods in which a pruning set is needed (REP and CCP) did not perform noticeably better than the other methods, and thus their requirement for additional data is a weakness. 
Mingers (1989a) compared five pruning methods, all of them presented in the previous sections (CCP, CVP, MEP, REP and PEP), and related them to different splitting measures. He states that pruning can improve the accuracy of induced decision trees by up to $25 \%$ in domains with noise and residual variation. In addition, he highlights the following findings: (i) MEP (the original version by Niblett and Bratko (1986)) is the least accurate method due to its sensitivity to the number of classes in the data; (ii) PEP is the most "crude" strategy, though the fastest one - due to some bad results, it should be used with caution; (iii) CVP, CCP and REP performed well, providing consistently low error-rates for all data sets used; and (iv) there is no evidence of an interaction between the splitting measure and the pruning method used for inducing a decision tree.

Buntine (1992a), in his $\mathrm{PhD}$ thesis, also reports experiments on pruning methods (PEP, MEP, CCP 0-SE and 1-SE for both pruning set and cross-validation). Some of his findings were: (i) CCP 0-SE versions were marginally superior than the 1-SE versions; (ii) CCP 1-SE versions were superior in data sets with little apparent structure, where more severe pruning was inherently better; (iii) CCP 0-SE with cross-validation was marginally better than the other methods, though not in all data sets; and (iv) PEP performed reasonably well in all data sets, and was significantly superior in well-structured data sets (mushroom, glass and LED, all from UCI (Frank and Asuncion, 2010));

Esposito et al. (1997) compare the six post-pruning methods presented in the previous sections within an extended C4.5 system. Their findings were the following: (i) MEP, CVP, and EBP tend to underprune, whereas 1-SE (both cross-validation and pruning set versions) and REP have a propensity for overpruning; (ii) using a pruning-set is not usually a good option; (iii) PEP and EBP behave similarly, despite the difference in their formulation; (iv) pruning does not generally decrease the accuracy of a decision tree (only one of the domains tested was deemed as "pruning-averse"); and (v) data sets not prone to pruning are usually the ones with the highest base error whereas data sets with a low base error tend to benefit of any pruning strategy.

For a comprehensive survey of strategies for simplifying decision trees, please refer to (Breslow and Aha, 1997). For more details on post-pruning techniques in decision trees for regression, we recommend (Breiman et al., 1984; Karalič, 1992; Osei-Bryson, 2008; Robnik-Sikonja and Kononenko, 1998; Torgo, 1997, 1998a,b).

\subsubsection{Missing Values}

Handling missing values (denoted in this section by “?”) is an important task not only in machine learning itself, but also in decision-tree induction. Missing values can be an issue during tree induction and also during classification. During tree induction, there are two moments in which we need to deal with missing values: splitting criterion evaluation and instances splitting.

During the split criterion evaluation in node $t$ based on attribute $a_{i}$, some common strategies are: 
- Ignore all instances belonging to the set $M=\left\{x_{j} \mid a_{i}\left(x_{j}\right)=\right.$ ? $\}$ (Breiman et al., 1984; Friedman, 1977);

- Imputation of missing values with the mode (nominal attributes) or the mean/median (numeric attributes) of all instances in $t$ (Clark and Niblett, 1989);

- Weight the splitting criterion value (calculated in node $t$ with regard to $a_{i}$ ) by the proportion of missing values, i.e., $|M| / N_{x}^{(t)}$ (Quinlan, 1989).

- Imputation of missing values with the mode (nominal attributes) or the mean/median (numeric attributes) of all instances in $t$ whose class attribute is the same of the instance whose $a_{i}$ value is being imputed (Loh and Shih, 1997).

For deciding which child node training instance $x_{j}$ should go to, considering a split in node $t$ over $a_{i}$, and that $a_{i}\left(x_{j}\right)=$ ?, some possibilities are:

- Ignore instance $x_{j}$ (Quinlan, 1986);

- Treat instance $x_{j}$ as if it has the most common value of $a_{i}$ (mode or mean/median) (Quinlan, 1989);

- Weight instance $x_{j}$ by the proportion of cases with known value in a given partition, i.e., $N_{x}^{\left(v_{l}\right)} /\left(N_{x}^{(t)}-|M|\right)$ (assuming $t$ is the parent node and $v_{l}$ is its $l^{\text {th }}$ partition) (Kononenko et al., 1984);

- Assign instance $x_{j}$ to all partitions (Friedman, 1977);

- Build an exclusive partition for missing values (Quinlan, 1989).

- Assign instance $x_{j}$ to the partition with the greatest number of instances that belong to the same class that $x_{j}$. Formally, if $x_{j}$ is labeled as $y_{l}$, we assign $x_{j}$ to $\arg \max _{v_{m}}\left[N_{v_{m}, y_{l}}\right]$ (Loh and Shih, 1997).

- Create a surrogate split for each split in the original tree based on a different attribute (Breiman et al., 1984). For instance, a split over attribute $a_{i}$ will have a surrogate split over attribute $a_{j}$, given that $a_{j}$ is the attribute which most resembles the original split. Resemblance between two attributes in a binary tree is given by:

$$
r e s\left(a_{i}, a_{j}, \mathbf{X}\right)=\frac{\left|\mathbf{X}_{\mathbf{a}_{\mathbf{i}} \in \mathbf{d}_{\mathbf{1}}\left(\mathbf{a}_{\mathbf{i}}\right) \wedge \mathbf{a}_{\mathbf{j}} \in \mathbf{d}_{\mathbf{1}}\left(\mathbf{a}_{\mathbf{j}}\right)}\right|}{N_{x}}+\frac{\left|\mathbf{X}_{\mathbf{a}_{\mathbf{i}} \in \mathbf{d}_{2}\left(\mathbf{a}_{\mathbf{i}}\right) \wedge \mathbf{a}_{\mathbf{j}} \in \mathbf{d}_{\mathbf{2}}\left(\mathbf{a}_{\mathbf{j}}\right)}\right|}{N_{x}}
$$

where the original split over attribute $a_{i}$ is divided in two partitions, $d_{1}\left(a_{i}\right)$ and $d_{2}\left(a_{i}\right)$, and the alternative split over $a_{j}$ is divided in $d_{1}\left(a_{j}\right)$ and $d_{2}\left(a_{j}\right)$. Hence, for creating a surrogate split, one must find attribute $a_{j}$ that, after divided by two partitions $d_{1}\left(a_{j}\right)$ and $d_{2}\left(a_{j}\right)$, maximizes $\operatorname{res}\left(a_{i}, a_{j}, \mathbf{X}\right)$. 
Finally, for classifying an unseen test instance $x_{j}$, considering a split in node $t$ over $a_{i}$, and that $a_{i}\left(x_{j}\right)=$ ?, some alternatives are:

- Explore all branches of $t$ combining the results. More specifically, navigate through all ambiguous branches of the tree until reaching different leaves and choose class $k$ with the highest probability, i.e., $\left.\arg \max _{y}\left[\sum_{s \in \lambda_{t}}\left[N_{\bullet}^{(s)}\right] / y_{l}\right] / N_{x}^{(t)}\right]$ (Quinlan, 1987a);

- Treat instance $x_{j}$ as if it has the most common value of $a_{i}$ (mode or mean/median);

- Halt the classification process and assign instance $x_{j}$ to the majority class of node $t$ (Quinlan, 1989).

\subsection{Other Induction Strategies}

We presented a thorough review of the greedy top-down strategy for induction of decision trees in the previous section. In this section, we briefly present alternative strategies for inducing decision trees.

Bottom-up induction of decision trees was first mentioned in (Landeweerd et al., 1983). The authors propose a strategy that resembles agglomerative hierarchical clustering. The algorithm starts with each leaf having objects of the same class. In that way, a $k$-class problem will generate a decision tree with $k$ leaves. The key idea is to merge, recursively, the two most similar classes in a non-terminal node. Then, a hyperplane is associated to the new non-terminal node, much in the same way as in top-down induction of oblique trees (in (Landeweerd et al., 1983), a linear discriminant analysis procedure generates the hyperplanes). Next, all objects in the new non-terminal node are considered to be members of the same class (an artificial class that embodies the two clustered classes), and the procedure evaluates once again which are the two most similar classes. By recursively repeating this strategy, we end up with a decision tree in which the more obvious discriminations are done first, and the more subtle distinctions are postponed to lower levels. Landeweerd et al. (1983) propose using the Mahalanobis distance to evaluate similarity among classes:

$$
\operatorname{dist}_{M}(i, j)^{2}=\left(\mu_{\mathbf{y}_{\mathbf{i}}}-\mu_{\mathbf{y}_{\mathbf{j}}}\right)^{T} \Sigma^{-1}\left(\mu_{\mathbf{y}_{\mathbf{i}}}-\mu_{\mathbf{y}_{\mathbf{j}}}\right)
$$

where $\mu_{\mathbf{y}_{\mathbf{i}}}$ is the mean attribute vector of class $y_{i}$ and $\Sigma$ is the covariance matrix pooled over all classes.

Some obvious drawbacks of this strategy of bottom-up induction are: (i) binary-class problems provide a 1-level decision tree (root node and two children); such a simple tree cannot model complex problems; (ii) instances from the same class may be located in very distinct regions of the attribute space, harming the initial assumption that instances from the same class should be 
located in the same leaf node; (iii) hierarchical clustering and hyperplane generation are costly operations; in fact, a procedure for inverting the covariance matrix in the Mahalanobis distance is usually of time complexity proportional to $O\left(n^{3}\right)^{5}$. We believe these issues are among the main reasons why bottom-up induction has not become as popular as top-down induction. For alleviating these problems, Barros et al. (2011b) propose a bottom-up induction algorithm named BUTIA that combines EM clustering with SVM classifiers. The authors later generalize BUTIA to a framework for generating oblique decision trees, namely BUTIF (Barros et al., 2013), which allows the application of different clustering and classification strategies.

Hybrid induction was investigated in (Kim and Landgrebe, 1991). The ideia is to combine both bottom-up and top-down approaches for building the final decision tree. The algorithm starts by executing the bottom-up approach as described above until two subgroups are achieved. Then, two centers (mean attribute vectors) and covariance information are extracted from these subgroups and used for dividing the training data in a top-down fashion according to a normalized sum-of-squared-error criterion. If the two new partitions induced account for separated classes, then the hybrid induction is finished; otherwise, for each subgroup that does not account for a class, recursively executes the hybrid induction by once again starting with the bottom-up procedure. Kim and Landgrebe (1991) argue that in hybrid induction "It is more likely to converge to classes of informational value, because the clustering initialization provides early guidance in that direction, while the straightforward top-down approach does not guarantee such convergence".

Several studies attempted on avoiding the greedy strategy usually employed for inducing trees. For instance, lookahead was employed for trying to improve greedy induction (Buntine, 1992b; Chou, 1991; Dong and Kothari, 2001; Murthy and Salzberg, 1995; Norton, 1989). Murthy and Salzberg (1995) show that one-level lookahead does not help building significantly better trees and can actually worsen the quality of trees induced. A more recent strategy for avoiding greedy decision-tree induction is to generate decision trees through evolutionary algorithms. The idea involved is to consider each decision tree as an individual in a population, which is evolved through a certain number of generations. Decision trees are modified by genetic operators, which are performed stochastically. A thorough review of decision-tree induction through evolutionary algorithms is presented in Chapter 4.

In a recent work, Basgalupp et al. (2011) propose a decision-tree induction algorithm (called Beam Classifier) that seeks to avoid being trapped in local-optima by doing a beam search during the decision tree growth. A beam search algorithm keeps track of $w$ states rather than just one. It begins with $w$ randomly generated states (decision trees). At each step, all the successors of the $w$ states are generated. If any successor is a goal state, the algorithm halts. Otherwise, it selects the $w$ best successors from the complete list, discards the other states in the list, and repeats this loop until the quality of the best current tree cannot be improved. An interesting fact regarding the

\footnotetext{
${ }^{5}$ For inverting a matrix, the Gauss-Jordan procedure takes time proportional to $O\left(n^{3}\right)$. The fastest algorithm for inverting matrices to date is $O\left(n^{2.376}\right)$ (the Coppersmith-Winograd algorithm).
} 
beam search algorithm is that if we set $w=1$ we are actually employing the greedy strategy for inducing decision trees.

Beam Classifier starts with $n$ empty decision trees (root nodes), where $n$ is the number of data set attributes, and each root node represents an attribute. Then, the algorithm selects the best $w$ trees according to a given criterion, and each one is expanded. For each expansion, the algorithm performs an adapted pre-order tree search method, expanding recursively (for each attribute) the leaf nodes from left to right. Thus, this expansion results in $t$ new trees,

$$
t=\sum_{i=1}^{w} \sum_{j=1}^{\left|\lambda_{i}\right|} m_{i j}
$$

where $w$ is the beam-width and $m_{i j}$ is the number of available attributes ${ }^{6}$ at the $j^{\text {th }}$ leaf node of the $i^{\text {th }}$ tree. Then, the algorithm selects once again the best $w$ trees considering a pool of $p$ trees, $p=w+t$. This process is repeated until a stop criterion is satisfied.

Other examples of non-greedy strategies for inducing decision trees include: (i) using linear programming to complement greedy-induced decision trees (Bennett, 1994); (ii) incremental and non-incremental restructuring of decision trees (Utgoff et al., 1997); (iii) skewing the data to simulate an alternative distribution in order to deal with problematic cases for decision trees (e.g., the parity-like function) (Page and Ray, 2003); and (iv) anytime learning of decision trees (Esmeir and Markovitch, 2007).

Even though a lot of effort has been employed in the design of a non-greedy decision-tree induction algorithm, it is still debatable whether the proposed attempts can consistently obtain better results than the greedy top-down framework. Most of the times, the gain in performance obtained with a non-greedy approach is not sufficient to compensate for the extra computational effort.

\subsection{Chapter Remarks}

In this chapter, we presented the main design choices one has to face when programming a decision-tree induction algorithm. We gave special emphasis to the greedy top-down induction strategy, since it is by far the most researched technique for decision-tree induction.

Regarding top-down induction, we presented the most well-known splitting measures for univariate decision trees, as well as some new criteria found in the literature, in an unified notation. Furthermore, we introduced some strategies for building decision trees with multivariate tests, the so-called oblique trees. In particular, we showed that efficient oblique decision-tree induction has to make use of heuristics in order to derive "good" hyperplanes within non-terminal nodes. We detailed the strategy employed in the OC1 algorithm (Murthy et al., 1993, 1994) for deriving

\footnotetext{
${ }^{6}$ Nominal attributes are not used more than once in a given subtree.
} 
hyperplanes with the help of a randomized perturbation process. Following, we depicted the most common stopping criteria and post-pruning techniques employed in classic algorithms such as CART (Breiman et al., 1984) and C4.5 (Quinlan, 1993), and we ended the discussion on top-down induction with an enumeration of possible strategies for dealing with missing values, either in the growing phase or during classification of a new instance.

We ended our analysis on decision trees with some alternative induction strategies, such as bottom-up induction and hybrid-induction. In addition, we briefly discussed work that attempt to avoid the greedy strategy, by either implementing lookahead techniques, evolutionary algorithms, beam-search, linear programming, (non-) incremental restructuring, skewing, or anytime learning. In the next chapters, we present an overview of evolutionary algorithms and hyper-heuristics, and review how they can be applied to decision-tree induction. 


\section{Evolutionary Algorithms and Hyper-Heuristics}

This chapter presents the basic concepts of evolutionary algorithms (EAs) and hyper-heuristics (HHs), which are computational techniques directly explored in this thesis.

EAs are well-known population-based metaheuristics. They have been employed in artificial intelligence over several years with the goal of providing the near-optimal solution for a problem that comprises a very large search space. A general overview of EAs is presented in Section 3.1.

HHs, in turn, are a recently new field in the optimisation research area, in which a metaheuristic - often an EA, and this is why these related concepts are reviewed together in this chapter - is used for searching in the space of heuristics (algorithms), and not in the space of solutions, like conventional metaheuristics. The near-optimal heuristic (algorithm) provided by a HHs approach can be further employed in several distinct problems, instead of relying on a new search process for each new problem to be solved. An overview of HHs is given in Section 3.2

\subsection{Evolutionary Algorithms}

Evolutionary algorithms (EAs) are a collection of optimisation techniques whose design is based on metaphors of biological processes. Freitas (2008) defines EAs as "stochastic search algorithms inspired by the process of neo-Darwinian evolution", and Weise (2009) states that "EAs are population-based metaheuristic optimisation algorithms that use biology-inspired mechanisms (...) in order to refine a set of solution candidates iteratively". 
The idea surrounding EAs is the following. There is a population of individuals, where each individual is a possible solution to a given problem. This population evolves towards increasingly better solutions through stochastic operators. After the evolution is completed, the fittest individual represents a "near-optimal" solution for the problem at hand.

For evolving individuals, an EA evaluates each individual through a fitness function that measures the quality of the solutions that are being evolved. After the evaluation of all individuals that are part of the initial population, the algorithm's iterative process starts. At each iteration, hereby called generation, the fittest individuals have a higher probability of being selected for reproduction to increase the chances of producing good solutions. The selected individuals undergo stochastic genetic operators, such as crossover and mutation, producing new offspring. These new individuals will replace the current population of individuals and the evolutionary process continues until a stopping criterion is satisfied (e.g., until a fixed number of generations is achieved, or until a satisfactory solution has been found).

There are several kinds of EAs, such as genetic algorithms (GAs), genetic programming (GP), classifier systems (CS), evolution strategies (ES), evolutionary programming (EP), estimation of distribution algorithms (EDA), etc. This chapter will focus on GA and GP, the most commonly used EAs for data mining (Freitas, 2002). At a high level of abstraction, GAs and GP can be described by the pseudocode in Algorithm 3.

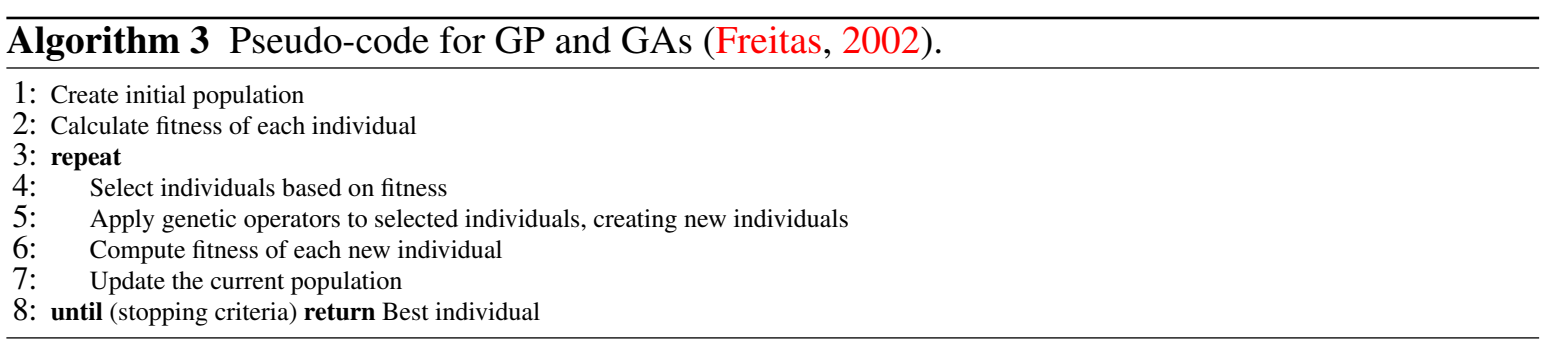

GAs, which were initially presented by Holland in his pioneering monograph (Holland, 1975), are defined as:

(...) search algorithms based on the mechanics of natural selection and natural genetics. They combine survival of the fittest among string structures [our italics] with a structured yet randomized information exchange to form a search algorithm with some of the innovative flair of human search. (p. 1)

Representation is a key issue in GAs, and while they are capable of solving a great many problems, the use of fixed-length character strings may not work on a variety of cases. John Koza, the researcher responsible for spreading the GP concepts to the research community, argues in his text book on GP (Koza, 1992b) that the initial selection of string length limits the number of internal states of the system and also limits what the system can learn. Moreover, he states that representation schemes based on fixed-length character strings do not provide a convenient way of representing computational procedures or of incorporating iteration or recursion when these capabilities are desirable or necessary to solve a given problem. Hence, he defines GP as: 
(...) a paradigm that deals with the problem of representation in genetic algorithms by increasing the complexity of the structures undergoing adaptation. In particular, the structures undergoing adaptation in genetic programming are general, hierarchical computer programs of dynamically varying size and shape. (p. 73)

After analysing the definitions of GAs and GP, it is not hard to see why the type of solution encoding in an EA is usually argued to determine the type of EA used. If solutions are encoded in a fixed-length linear string, researchers claim a GA is being used. Conversely, tree-encoding schemes usually imply the use of GP. Although solution encoding can differentiate between GAs and GP, the main question perhaps is not what the representation is (e.g.a linear string or a tree) but rather how the representation is interpreted (Basgalupp et al., 2009b).

In this sense, Woodward (2003) recommends defining GAs and GP according to the genotype-phenotype mapping: if there is a one-to-one mapping, the EA in question is a GA; if there is a many-to-one mapping, the EA is a GP. Nevertheless, this definition is tricky. For instance, assume a feature selection problem in data mining, where an individual (chromosome) consists of $n$ genes, one for each attribute. Now assume that each gene contains a real value in the range $[0,1]$, representing the probability of the corresponding attribute being selected. Assume also that, for decoding a chromosome, a threshold is predefined, and an attribute is selected only if the value of its gene is larger than that threshold. In this case, we have a many-to-one mapping, because there are many different genotypes (different arrays of probabilities) that may be decoded into the same phenotype (the same set of selected features). This particular many-to-one mapping does not indicate we are dealing with GP. Actually, we can use the same set of genetic operators and remaining parameters of a typical GA for this scenario. One may say that a good distinction between GAs and GP is whether a solution encodes data only (GAs) or data and functions (GP).

In this thesis, we present in Chapter 5 an EA that automatically designs decision-tree algorithms. It encodes its individuals as integer vectors and employs the same set of genetic operators and remaining parameters of a typical GA. However, it encodes both data (values of parameters) and functions (design components of decision-tree algorithms such as pruning methods). Given the difficulty in clearly defining whether the proposed approach is a GA or a GP algorithm, we henceforth adopt the generic term "evolutionary algorithm" when referring to our method. In the next few sections, we review the basic concepts involved when designing an EA: (i) individual representation and population initialization; (ii) fitness function; and (iii) selection methods and genetic operators.

\subsubsection{Individual Representation and Population Initialization}

The first step for designing an EA is to define how its individuals are going to be represented (encoded). Since each individual is a target solution to the problem at hand, so if we want to discover the best decision-tree induction algorithm for a given set of data sets, each individual should be a candidate decision-tree induction algorithm. 
It is during the step of deciding the individual encoding scheme that a link must be created between the "real world" and the "evolutionary world" (Eiben and Smith, 2003). Thus, the encoding scheme creates the bridge between the original optimisation problem and the search space to be explored by the EA.

The objects that represent possible solutions within the context of the original problem are referred to as phenotypes, whereas their encoding (the EA's individuals) are referred to as genotypes. Note that the phenotype search space may differ significantly from the genotype search space. A genotype must be decoded so we can have a clear notion of its performance as a candidate solution to the original problem.

Defining a suitable individual encoding scheme is not an easy task, and it may be decisive for the success of the EA. As we will see in the next few sections, there is a direct relation between individual representation and the genetic operators that guide the evolutionary process. Finding suitable encoding schemes for the problem at hand is a task that usually comes with practical experience and solid knowledge on the application domain (Mitchell, 1998).

GA individuals, as previously seen, are encoded as fixed-length character strings such as binary or integer vectors. GP individuals, on the other hand, are usually represented in a tree-based scheme (a consequence of the GP definition by Koza). Notwithstanding, there are also studies that encode GP individuals as linear structures, and even graphs (Banzhaf et al., 1998). In Figure 3.1, we have a GP individual that comprises four terminals $(a, b, c, d)$ and three functions $(O R,-, \times)$.

a)

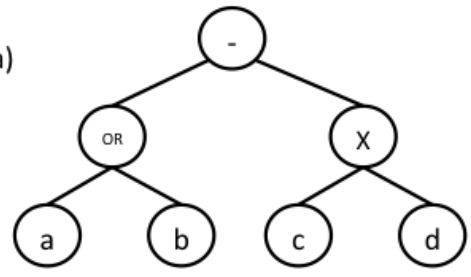

b)

\begin{tabular}{|l|l|l|l|l|l|l|}
\hline$a$ & OR & $b$ & - & $c$ & $x$ & $d$ \\
\hline
\end{tabular}

c)

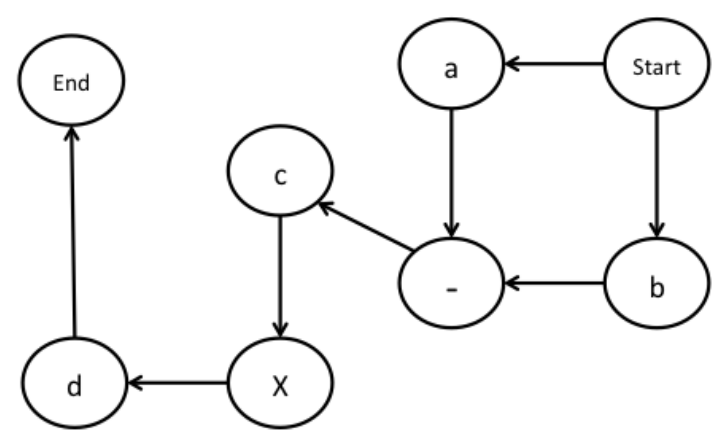

Figure 3.1: A GP individual represented as: a) a tree; b) a linear structure and c) a graph.

The initialization of individuals in a GA is usually completely random, i.e., a random value within the range of accepted values is generated for each gene (position of the vector) of the individual's genome. Nevertheless, domain knowledge may be inserted in order to guarantee some robustness to the individuals of the initial population. 
The initialization of individuals in GP offers more possibilities due to its usually-adopted tree structure. Koza (1992b) proposes two distinct methods (both in the context of tree representation): full and grow. The full method works as follows: until the maximum initial depth of the tree is reached, randomly choose non-terminals nodes; when the maximum depth is reached, randomly choose terminal nodes. This method leads to trees whose branches are of equal size. Alternatively, the grow method allows the choice of either non-terminals and terminals (except for the root node which is always a non-terminal). Hence, the trees generated may have irregular shapes, because terminal nodes end the growth of the branch even if the maximum depth has not yet been reached. The grow method is said to have serious weakness, such as (Luke, 2000): (i) the method picks all functions with equal likelihood; there is no way to fine tune the preference of certain functions over others; (ii) the method does not give the user much control over the tree structures generated; and (iii) while the maximum depth parameter (or, alternatively, the maximum number of nodes) is used as an upper bound on maximal tree depth, there is no appropriate way to create trees with either a fixed or average tree size or depth. The full method, in turn, is criticized for producing a very narrow range of tree structures.

A third option to generate trees is the ramped half-and-half method (RHH). It is intended to enhance the diversity of individuals by generating, for each depth level, half of the trees through the grow method and half of the trees through the full method. Burke et al. (2003a) draw some considerations on the bias of the RHH method, though most of their analysis is inconclusive. The only known fact is that RHH tends to generate more trees through the full method. This occurs because duplicates are typically not allowed, and the grow method tends to generate smaller trees (hence it is more susceptible to generate duplicates).

Other tree-generation algorithms for genetic programming include probabilistic tree-creation 1 and 2 (PTC 1 and PTC 2) (Luke, 2000), RandomBranch (Chellapilla, 1997) and exact uniform initialization (Bohm and Geyer-Schulz, 1996), just to name a few.

\subsubsection{Fitness Function}

After initializing the population, an EA evaluates the goodness of each individual. A fitness function is responsible for evaluating how well an individual solves the target problem. It is mainly problem-dependent, i.e., different problems tend to demand different fitness functions. For instance, in a problem where each individual is a decision tree, an interesting fitness function could be the accuracy of the given tree in a training/validation data set. Similarly, if the individual is a decision-tree induction algorithm, an interesting fitness function could take into account both the time complexity of the individual and the performance of the trees it generates for a set of data sets. Good individuals have a higher probability of being selected for reproduction, crossover and mutation (detailed in Section 3.1.3), and thus have their genetic material perpetuated through the evolutionary process. 
One of the advantages EAs is that their "black-box" character makes only few assumptions about the underlying fitness functions. In other words, the definition of fitness functions usually requires lesser insight to the structure of the problem space than the manual construction of an admissible heuristic. This advantage enables EAs to perform consistently well in many different problem domains (Weise, 2009). Regarding the machine learning context, many algorithms are only capable of evaluating partial solutions (Freitas, 2008). For instance, a conventional greedy decision-tree induction algorithm incrementally builds a decision tree by partitioning one node at a time. When the algorithm is evaluating several candidate divisions, the tree is still incomplete, being just a partial solution, so the decision tree evaluation function is somewhat shortsighted. A GP can evaluate the candidate solution as a whole, in a global fashion, through its fitness function.

The fitness evaluation can be carried out by searching for individuals that optimise a single measure (i.e., single-objective evaluation), or by optimising multiple objectives (i.e., multi-objective evaluation). Multi-objective evaluation is quite useful in the context of machine learning. For instance, it is often desirable to obtain decision trees that are both accurate and comprehensible, in the shortest space of time. Several approaches for handling multi-objective problems were proposed in the literature, such as the weighted-formula, Pareto dominance, and the lexicographic analysis (Coello, 1999; Coello et al., 2006)).

\subsubsection{Selection Methods and Genetic Operators}

After evaluating each individual, a selection method is employed for deciding which individuals will undergo reproduction, crossover and mutation. Some well-known selection methods are fitness-proportional selection, tournament selection, and linear ranking selection (Back, 1994; Blickle and Thiele, 1995).

In fitness-proportional selection, each individual $i$ has probability $p_{i}$ of being selected, which is proportional to its relative fitness, i.e., $p_{i}=f(i) / \sum_{j=1}^{I} f(j)$, where $f($.$) is the fitness function and$ $I$ is the total number of individuals in the GP population. In tournament selection, $t$ individuals are selected at random from the population and the best individual from this $t$-group is selected. A common value for tournament size $t$ is 2 (Goldberg and Deb, 1991). In linear ranking selection, individuals are first ranked according to their fitness and then selected based on the value of their rank positions. This method overcomes the scaling problems of fitness-proportional assignment, e.g., premature convergence when few individuals with very high fitness values dominate the rest of the population.

Once the individuals have been selected, they undergo genetic operators such as reproduction (individual is cloned into the next generation), crossover (swapping of genetic material from two individuals, generating offspring) and mutation (modification of the genetic material of an individual).

There are many different ways to implement the crossover and mutation operators, and they also vary according to the individual encoding scheme. The standard EA crossover works by 
recombining the genetic material of two parent individuals, resulting in the generation of two children. For GAs, the most widely-used crossover strategies are one-point crossover, two-point crossover, and uniform crossover. In one-point crossover, the parents' linear strings are divided into two parts (according to the selected "point"), and the offspring is generated by using alternate parts of the parents. In two-point crossover, the rationale is the same but this time the string is divided into three, according to two distinct "points". Finally, uniform crossover is performed by swapping the parents genes in a per-gene fashion, given a swap probability. Figure 3.2 depicts these three crossover strategies usually adopted by GAs.

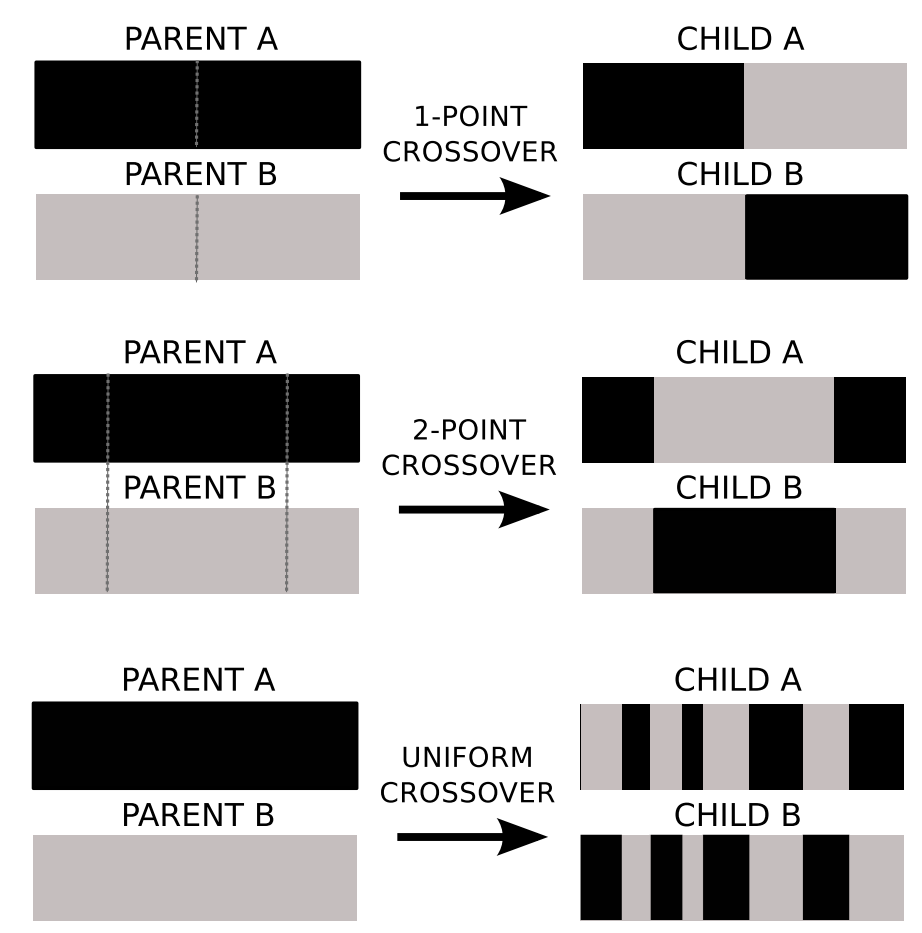

Figure 3.2: Crossover strategies for linear strings: a) one-point crossover; b) two-point crossover; and c) uniform crossover.

Mutation in linear genomes is usually performed in a per-gene basis, though there are exceptions such as the flip bit mutation, in which a binary vector is inverted ( 1 is changed to 0 , and 0 is changed to 1 ). Usually, either a particular gene is selected to undergo mutation, or the entire genome is traversed and each gene has a probability of undergoing mutation. In integer and float genomes, operators such as non-uniform, uniform, and Gaussian mutation may be employed. Non-uniform mutation is employed with higher probability in the initial steps of evolution, to avoid the population from stagnating, and with lower probability in the later stages, only to fine-tune the individuals. Uniform mutation replaces the value of each gene with a random uniform value selected within the gene's range. Finally, Gaussian mutation adds a unit Gaussian-distributed random value to the chosen gene, and values outside the gene's boundaries are clipped.

In tree-based encoding schemes (GP), crossover occurs by swapping randomly chosen subtrees from the two parents. Regarding mutation, the standard approach is to replace a randomly selected 
individual's subtree by a randomly generated one. Even though crossover is the predominant operator in GP systems (Banzhaf et al., 1998), there is a large controversy whether GP crossover is a constructive operator or a disruptive one. A constructive operator propagates good genetic material (good building blocks), increasing the quality of individuals across generations. A disruptive operator is a search strategy that mostly harms good solutions instead of improving them. In short, since GP crossover swaps subtrees from random positions in the tree, the larger the "good" building block contained in a tree, the higher the probability of it being disrupted after future crossover operations. This is the main reason for some researchers to claim that standard GP crossover is in fact a macromutation operator ${ }^{1}$. As a result of this controversy, new intelligent (context-aware, semantically-based, etc.) crossover operators are suggested for avoiding the negative effect of standard GP crossover (see, for instance, (Majeed and Ryan, 2006a,b; Uy et al., 2010)).

\subsection{Hyper-Heuristics}

Metaheuristics such as tabu search, simulated annealing, and EAs, are well-known for their capability of providing effective solutions for optimisation problems. Nevertheless, they require expertise to be properly adopted for solving problems from a particular application domain. Moreover, there are drawbacks that prevent metaheuristics to be easily applied to newly encountered problems, or even new instances of known problems. These drawbacks arise mainly from the large range of parameter or choices involved when using this type of approaches, and the lack of guidance as to how to proceed for selecting them (Burke et al., 2009).

Hyper-heuristics (HHs) operate on a different level of generality from metaheuristics. Instead of guiding the search towards near-optimal solutions for a given problem, a HHs approach operates on the heuristic (algorithmic) level, guiding the search towards the near-optimal heuristic (algorithm) that can be further applied to different application domains. HHs are therefore assumed to be problem independent and can be easily utilised by experts and non-experts as well (Özcan et al., 2008). A hyper-heuristic can be seen as a high-level methodology which, when faced with a particular problem instance or class of instances, and a number of low-level heuristics (or algorithm's design components), automatically designs a suitable combination of the provided components to effectively solve the respective problem(s) (Burke et al., 2009).

Figure 3.3 illustrates the different generality levels in which metaheuristics and hyper-heuristics work in. Note that whereas metaheuristics perform the search in the space of candidate solutions, hyper-heuristics perform the search in the space of candidate heuristics (algorithms), which in turn generate solutions for the problem at hand. To illustrate this rationale, let us compare two different evolutionary approaches in decision-tree induction. In the first approach, an EA is used to evolve

\footnotetext{
${ }^{1}$ For instance, Angeline (1997) argues that the lack of performance of standard GP crossover (in comparison to some types of macromutations) indicates that it is, in effect, more accurately described as a population-limited macromutation operation rather than an operation consistent with the building block hypothesis.
} 
the best decision tree for the postoperative-patient UCI data set (Frank and Asuncion, 2010). In the second approach, an EA is used to evolve the best decision-tree algorithm to be further applied to medical data sets. Observe that, in the the first approach, the EA works as a metaheuristic, because it searches for the best decision-tree to the postoperative-patient data. Therefore, the ultimate goal is to achieve an accurate decision tree for this particular problem. In the second approach, the EA works as a hyper-heuristic, because it searches for the best decision-tree algorithm, which in turn generates decision trees that can be applied to several different instances of medical applications. Note that the second approach is problem independent - instead of generating a decision tree that is only useful for classifying patients from the postoperative-patient data set, it generates a decision-tree algorithm that can be applied to several medical data sets, including the postoperative patient one.

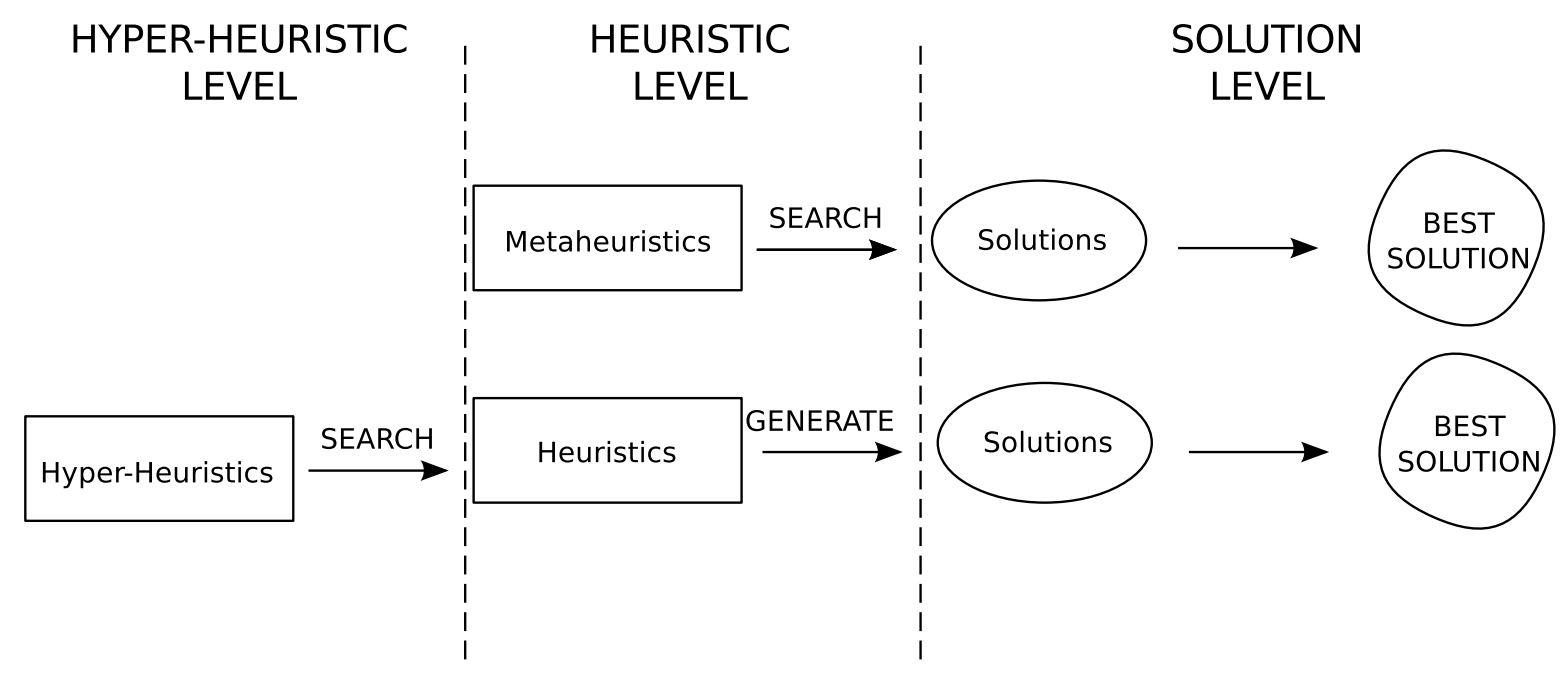

Figure 3.3: Metaheuristics versus hyper-heuristics.

The term hyper-heuristic is reasonably new, as it first appeared in a conference paper in 2000 (Cowling et al., 2001b), and in a journal paper in 2003 (Burke et al., 2003b). However, the methodology surrounding HHs are not new. It can be traced back to as early as the 1960s, when Fisher and Thompson (1963) proposed the combination of scheduling rules (also known as priority or dispatching rules) for production scheduling, claiming that it would outperform any of the rules taken separately. This pioneering work was developed in a time when metaheuristics were not a mature research area. Even so, the proposed learning approach based on probabilistic learning resembles a stochastic local-search algorithm that operates in the space of scheduling rules sequences.

Most of the hyper-heuristic research aims at solving typical optimisation problems, such as the previously-mentioned production scheduling problem (Fisher and Thompson, 1963; Ochoa et al., 2009b; Storer et al., 1992; Vázquez-Rodríguez and Petrovic, 2010), and also educational timetabling (Burke et al., 2007, 2006; Ochoa et al., 2009a; Pillay, 2008), 1D packing (Marín-Blázquez and Schulenburg, 2007), 2D cutting and packing (Garrido and Riff, 2007), 
constraint satisfaction (Terashima-Marín et al., 2010), and vehicle routing (Garrido and Castro, 2009; Garrido and Riff, 2010).

Applications of hyper-heuristics in machine learning are not that frequent and much more recent than optimisation applications. Examples of HHs approach in machine learning include the work of Stanley and Miikkulainen (2002), which proposes an evolutionary system for optimising the neural network topology; the work of Oltean (2005), which proposes the evolution of evolutionary algorithms through a steady-state linear genetic programming approach; the work of Pappa and Freitas (2009a), covering the evolution of complete rule induction algorithms through grammar-based genetic programming; and the work of Vella et al. (2010), which proposes the evolution of heuristic rules in order to select distinct split criteria in a decision-tree induction algorithm. In Chapter 5, we present a hyper-heuristic approach for evolving complete decision-tree induction algorithms.

\subsection{Chapter Remarks}

In this chapter, we presented the basic concepts regarding EAs and HHs. First, we showed the basic concepts of evolutionary computation from the perspective of genetic algorithms and genetic programming, the two most employed EAs in data mining and knowledge discovery (Freitas, 2002). In particular, we briefly described the main individual representations (encoding schemes) for the design of an EA, and also briefly discussed on the importance of fitness evaluation during the evolutionary cycle. Furthermore, we detailed the most common selection methods and genetic operators employed by researchers from this area.

In the second part of this chapter, we introduced the reader to hyper-heuristics. More specifically, we defined the differences between a metaheuristic approach and a hyper-heuristic approach, by providing an example that relates evolutionary algorithms and decision-tree induction. In addition, we presented the origins of hyper-heuristic research, and cited its main applications in the areas of optimisation and machine learning.

In the next chapter (Chapter 4), we review the literature that combines EAs and decision trees. Following, in Chapter 5, we present our approach for evolving full decision-tree induction algorithms through a hyper-heuristic evolutionary algorithm. 
CHAPTER

4

\section{Evolutionary Algorithms for Decision-Tree Induction}

In this chapter, we present a survey of studies which relate evolutionary algorithms and decision trees. It is self-contained, and it reviews work that acknowledge the problems with the local search performed by the greedy-strategy usually employed for growing decision trees. It should be noticed that the papers presented in this chapter evolve decision trees or particular decision-tree components, and not decision-tree induction algorithms, which is the main purpose of this thesis.

\subsection{Introduction}

We have seen in Chapter 2 that most decision-tree induction algorithms are based on a greedy top-down recursive partitioning strategy for tree growth. They use different variants of impurity measures, like, information gain (Quinlan, 1986), gain ratio (Quinlan, 1993), gini-index (Breiman et al., 1984) and distance-based measures (De Mántaras, 1991), to select an input attribute to be associated with an internal node. One major drawback of greedy search is that it usually leads to sub-optimal solutions. Moreover, recursive partitioning of the data set may result in very small data sets for the attribute selection in the deepest nodes of a tree, which in turn may cause data overfitting.

Several alternatives have been proposed to overcome these problems, including the induction of an ensemble of trees. Ensembles are created by inducing different trees from training samples and the ultimate classification is frequently given through a voting scheme (see (Hastie et al., 2009; Seni and Elder, 2010)). However, a disadvantage of ensembles is that the comprehensibility 
of analysing a single decision tree is lost. Indeed, classification models being combined in an ensemble are often, to some extent, inconsistent with each other; an inconsistency that is necessary to increase the predictive accuracy of the ensemble (Freitas et al., 2010). Therefore, ensembles are not a good option for applications where comprehensibility is crucial.

Hence, an approach that has been increasingly used is the induction of decision trees through Evolutionary Algorithms (EAs). We have seen in Chapter 3 that, instead of a local search, EAs performs a robust global search in the space of candidate solutions. As a result, they tend to cope better with attribute interactions than greedy methods (Freitas, 2002).

The number of EAs for decision-tree induction has grown in the past few years, mainly because they report good predictive accuracies whilst keeping the comprehensibility of decision trees. In this context, we provide a detailed survey of EAs to evolve decision trees for classification (Section 4.3) and regression (Section 4.4). Furthermore, we discuss EAs designed to improve specific components of decision tree classifiers (Section 4.5). For instance, we discuss EAs for finding the optimal hyperplane in oblique decision trees (Chai, 1996; Chai et al., 1996; Kim et al., 2004a,b), and for improving tree pruning (Chen et al., 2009; Shah and Sastry, 1999) and other components related to decision-tree induction (Bratu et al., 2007; Turney, 1995). Finally, we review applications of evolutionary algorithms for decision-tree induction in different domains, such as software estimation (Burgess and Lefley, 2001), software modules protection (Podgorelec and Kokol, 2002) and cardiac imaging data (Cuong and Pham, 2009) (Section 4.6). We end this chapter addressing some important issues and open questions for future research (Section 4.7).

It is important to stress that comprehensive surveys on decision trees have been previously published, such as the papers by Safavian and Landgrebe (1991), Murthy (1998), and Rokach and Maimon (2005). Also, another paper has been recently published addressing EAs in classification (Espejo et al., 2010). Nevertheless, to the best of the author's knowledge, none of them has been fully devoted to address evolutionary induction of decision trees for classification and regression, as well as their respective applications.

\subsection{Taxonomy}

We propose a taxonomy of EAs for decision-tree induction that is divided into two main threads: (i) evolutionary induction of decision trees; and (ii) evolutionary design of decision tree-components. This taxonomy is presented in Figure 4.1. In (i), each individual of the evolutionary algorithm is a decision tree, whereas in (ii) individuals are components of decision tree classifiers.

Evolutionary induced decision trees for classification can be either axis-parallel, when there is a single attribute that splits the training data per node, or oblique, when there is a (non-) linear combination of attributes per split. Decision trees for regression can be divided into regression 


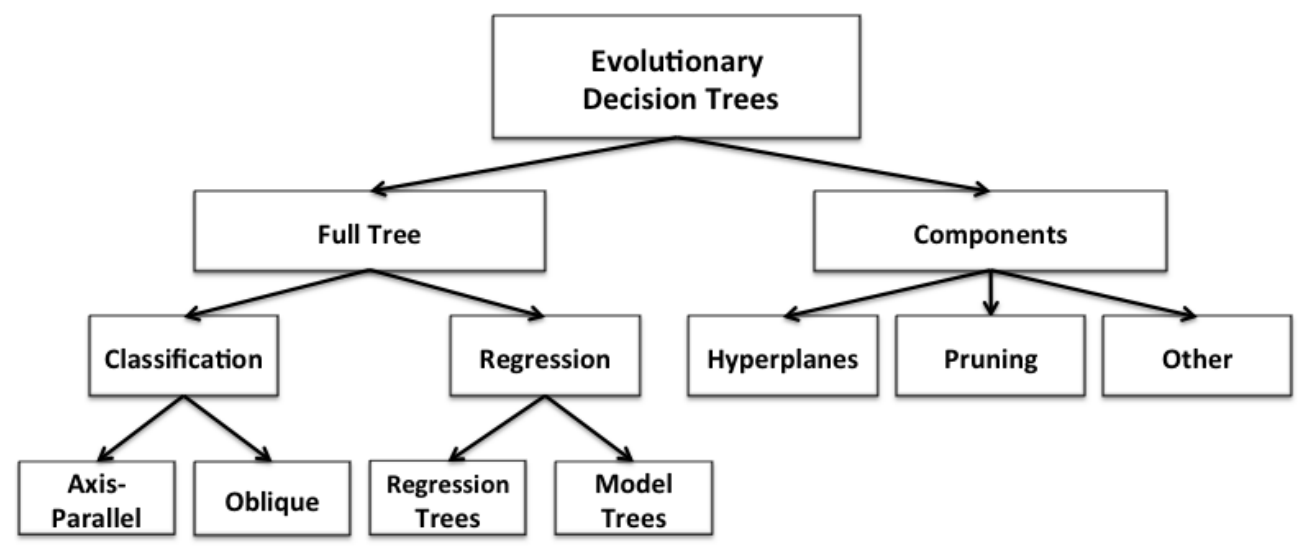

Figure 4.1: Taxonomy of evolutionary algorithms for decision-tree induction.

trees, when each leaf-node assigns a value to a test instance, and model trees, when the leaf-nodes contain (non-) linear regression models that are used to predict a value for a new test instance.

Evolutionary design of components can be divided into:

- Hyperplane evolution, where, at each tree node, an EA evolves a near-optimal (non-) linear combination of attributes for oblique trees;

- Pruning method evolution, where an EA is used to handle pruning over a induced decision tree;

- Evolution of other methods, such as parameters of the impurity measure used to split nodes.

Next, we discuss EAs which are part of the first thread of our taxonomy: evolutionary induction of decision trees, starting with decision trees for classification.

\subsection{Classification}

This section reviews EAs that evolve decision trees for classification tasks, where each individual is a classification tree. We divide this section into two parts: (i) EAs that evolve axis-parallel decision trees; and (ii) EAs that evolve oblique decision trees.

\subsubsection{Axis-Parallel Decision Trees}

Axis-parallel decision trees are the most common type found in the literature, mainly because this type of tree is usually much easier to interpret than an oblique tree. We divide our analysis on axis-parallel decision trees according to the main steps of the evolutionary process. That is, we analyse how solutions are encoded; which methods are used for initializing the population of decision trees; the most common strategies for fitness evaluation; the genetic operators that are designed to evolve individuals; and other related issues. 


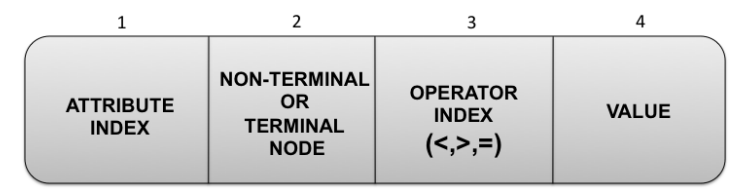

Figure 4.2: Node representation in tree-encoding (Aitkenhead, 2008). A tuple of 4 elements that defines (1) the data set attribute to be tested or predicted; (2) the type of node (non-terminal or terminal); (3) the operator to be used; and (4) the value to be tested by the attribute in (1) according to the operator in (3) or, alternately, the binary classification value.

\section{Solution Encoding}

Tree-based encoding is the most common approach for coding individuals in EAs for decision-tree induction, and it seems a natural choice when we are dealing with decision trees. In (Aitkenhead, 2008), the author applies competitive co-evolution for decision-tree induction and uses a tree-encoding scheme. The system designs binary decision trees where each node is represented by a 4-tuple (Figure 4.2). Each component in Figure 4.2 is a numeric (integer or real) value that can be modified during the evolutionary process. The first element of the 4-tuple is an integer that indexes each data set attribute; the second one is an integer that indicates whether the node is non-terminal or terminal; the third one is an integer that indexes which operator is to be used $(<,>,=)$; and the fourth one is a real number that indicates the value to be tested (in a non-terminal node) or the binary classification (in a terminal node). It is not clear how the linkage of nodes is handled, but we assume each node described in Figure 4.2 has two pointers for the children nodes (which assume null values for terminal nodes).

A similar approach is presented in (Shirasaka et al., 1998; Tanigawa and Zhao, 2000; Zhao and Shirasaka, 1999), where the authors use a tree-encoding solution in which each node is a 7-tuple: node $=\{t$, label, $P, L, R, C$, size $\}$, where $t$ is the node number ( $\mathrm{t}=0$ is the root node), label is the class label of a terminal node (meaningful only for terminal nodes), $P$ is a pointer for the parent node, $L$ and $R$ are pointers to the left and right children, respectively (null for terminal nodes), and $C$ is a set of registers, where $C[0]$ stores the attribute id and $C[1]$ the threshold value for the test feature $_{C[0]}<C[1]$, whose possible outcomes are "yes" (path to the left-child node) or "no" (path to the right-child node).

Zhao's genetic programming system (Zhao, 2007) also encodes individuals as trees. Zhao defines terminal and function nodes. Terminals can be integer (attribute ids), real (attribute values) or binary (a class label or a function node) values. Each function node takes four arguments and returns a binary result. Its signature is $N:$ integer $\times$ real $\times$ binary $\times$ binary $\rightarrow$ binary. It can be represented as a four-tuple, $N=\{a, v, L, R\}$, where $a$ is an attribute terminal, $v$ is a value terminal, and $L$ and $R$ are class terminals or node functions. Note that since both the type of a class terminal and the output type of a node function are binary, $L$ and $R$ can be either class terminals or function nodes. 
Papagelis and Kalles (2001) defend the use of the tree-encoding scheme in EAs for decision-tree induction. They state that whereas GAs use binary strings to represent points in search space, such representations do not appear well suited for representing the space of variable-size decision trees. Furthermore, they affirm that it is natural to use a tree structure to represent decision trees and that the mutation-crossover operators can be efficiently altered to match this structure. For other tree-encoding scheme examples, see (Barros et al., 2010, 2011c; Basgalupp et al., 2009a,b; Fu et al., 2003a, 2006, 2003b; Fu and Mae, 2001; Podgorelec and Kokol, 2002; Sorensen and Janssens, 2003).

Even though the tree-encoding scheme is the most used approach for decision tree evolution, several studies use different approaches for coding individuals. Most of these approaches adopt a fixed-length string representation, an inheritance from the misconception that GAs are defined by their representations. Fixed-length string representations, also called "linear chromosomes", are typically tricky to implement for non-binary decision trees. Thus, most papers propose EAs that evolve binary decision trees, arguing, for instance, that any non-binary decision tree can be converted into a binary tree.

In (Kennedy et al., 1997), linear chromosomes are composed by genes, named caltrops, an allusion to the spiked devices used in medieval warfare to disable charging horses. Caltrops are subtrees formed by a root node and two child nodes. Each caltrop is represented by three integers, where non-terminal nodes are identified by an attribute index and terminal nodes by the value 0 (zero). Figure 4.3 shows how a decision tree (a) is divided into subtrees, which in turn are mapped into triples of integers named caltrops (b), genes that will form the individual's chromosome (c). Notice that caltrops do not encode tests over the attributes. This representation assumes that each data set attribute is boolean, and assigns the left (right) child node when the attribute value is true (false).

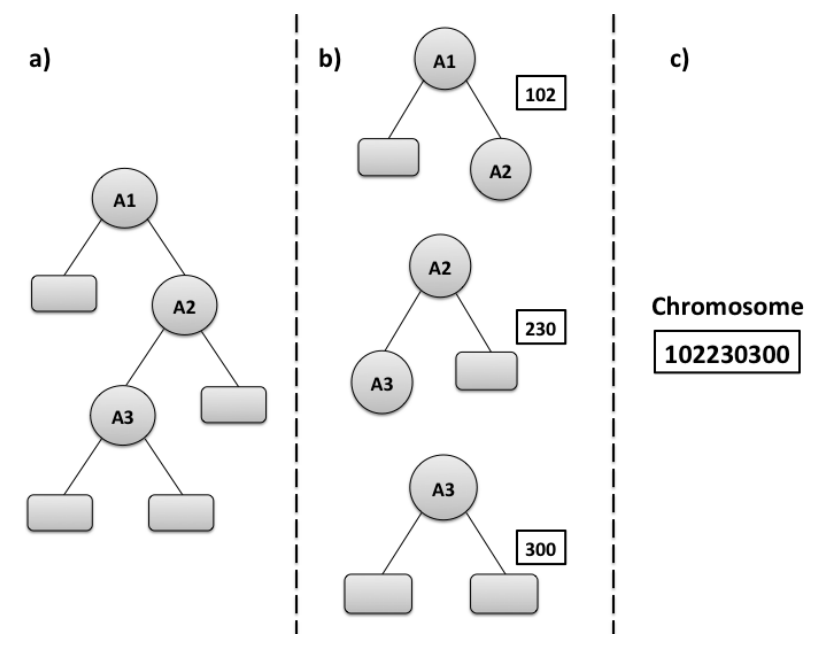

Figure 4.3: Mapping decision trees in linear chromosomes (Kennedy et al., 1997). a) decision tree. b) subtrees mapped into caltrops. c) chromosome resulting from the union of caltrops. 
Bandar et al. (1999) provide a GA for induction of multiple decision trees. Linear chromosomes are formed by integers that correspond to attribute indexes. The total number of genes in a chromosome is $2^{\text {depth }}-1$, where depth is the maximum number of attribute split levels, a variable parameter. To create the decision tree, one must select the attribute corresponding to the first gene of the chromosome. This attribute will be used to split the root node in left and right children. Afterwards, a binary branching is performed using the training set instances contemplated by the node (for the root node, all instances are used). The measure used to perform the binary branching is not mentioned in their work. The procedure is recursive, and the binary branching is performed until the tree has reached its maximum depth.

Smith (2008) also designs binary decision trees coded through linear chromosomes. Even though his strategy for evolving decision trees is domain-specific (RNA search acceleration), it could easily be extended to a generic framework. Each gene of the chromosome consists of two integers: (i) a node type $n$ (in reality the correspondent to an attribute index), which varies in the interval $[1,5]$ (domain constraint), and (ii) a type/index $v$, which is the threshold value for which the test $n \geq v$ is performed. Once again, the instances resulting from the test are filtered to the left (right) node if the result is false (true).

In spite of the fact that decision trees encoded as linear chromosomes seem to be easier to handle than those encoded by tree-encoding schemes, fixed-length linear chromosomes have some disadvantages, such as: (i) the need of constant mapping between genotype and phenotype for fitness evaluation; (ii) difficulty in handling non-binary decision trees and (iii) difficulty in defining a maximum number of genes for fixed-length structures. For instance, chromosomes with a large number of genes may have several genes with null values, which can in turn harm operations like one-point crossover. Conversely, structures with a low number of genes can ultimately restrain the size of the trees to be discovered in the evolutionary process. Dynamic-length string structures, on the other hand, may add an unnecessary complexity to the EA design. Genetic operations, like crossover, may have to be modified when chromosomes with different sizes are to be reproduced.

\section{Population Initialization}

An EA's initial population has to provide enough diversity of individuals so that the genetic operators can search for solutions in a more comprehensive search-space, avoiding local optima. Nonetheless, a large search-space may result in very slow convergence, preventing the EA from finding a near-optimal solution. In this case, task-dependent knowledge constraints may speed-up convergence by avoiding the search in "dead zones" of the solution space. It is clear that there is a thin line between the precise amount of diversification for avoiding local optima and task-dependent knowledge constraints for speeding-up convergence.

Most studies on evolutionary induction of decision trees propose a partially random initialization of trees. We use the term "partially" because the randomness of individuals is constrained to the data set attributes and their possible values within the training set. Some 
approaches propose additional constraints during initialization in order to to guarantee the logic validity of the created decision trees. For instance, DeLisle and Dixon (2004) state that their method initializes decision trees "at random based upon the training set and given mild constraints on the minimum number of observations allowed in terminal or leaf nodes".

For the cases where each decision tree is encoded as a fixed-length string, a random initialization is often used (Cha and Tappert, 2009; Kennedy et al., 1997; Reynolds and Al-Shehri, 1998). When the decision tree is encoded as a tree, a common strategy for initialization is randomly choosing attributes and split values from a predefined list and halting the decision tree growth when the tree reaches a depth that is randomly selected from an interval, typically [2, max Depth]. This strategy usually creates fully balanced trees (the distance from the root to any leaf node is the same). This generation procedure is called the full method (Kretowski and Grzes, 2006b; Shirasaka et al., 1998; Tanigawa and Zhao, 2000; Zhao, 2007; Zhao and Shirasaka, 1999). Trees can be generated with varying distances from root to the leaves, namely the grow method (Llora and Garrell, 2001). Finally, the population can be a mixture of trees generated by either the full or the grow method, a procedure named ramped half and half (Eggermont et al., 2003, 2004a,b; Garcia-Almanza and Tsang, 2006; Johansson and Niklasson, 2009). Random initialization of decision trees encoded as trees is further discussed in (Folino et al., 2000, 2001, 2002; Kim, 2004; Sorensen and Janssens, 2003).

Another approach for initializing decision trees in an EA is to restrain the initial population to 2-level decision trees, i.e., a root node and its respective leaves (Aitkenhead, 2008; Papagelis and Kalles, 2000, 2001; Rouwhorst and Engelbrecht, 2000). EAs that implement this strategy rely on the further application of genetic operators to obtain deeper trees.

One could say the only task-dependent knowledge that the previously commented EAs employ is related to the choice of possible split values. Although most of them claim to randomly choose split values, most of the times these values are not really random, i.e.they are a set of observed values from the training set. This is the most rudimentary use of task-dependent knowledge in the initialization process. For instance, Ma and Wang (2009) reduce the impact of randomness by backtracking a "bad" random choice and replacing it by a "better" random choice. The definition of good and bad choices, in this case, is given by the amount of training data that are filtered to each child node of the currently generated node. If no random choice is considered to be good enough, a leaf node stops the growth of that particular path.

A more robust approach for generating task-dependent knowledge-based initial trees is executing a greedy traditional algorithm, e.g.C4.5 or CART, in samples from the training set and incorporating the resulting decision trees in the initial population. This strategy is implemented in (Fu et al., 2006; Fu and Mae, 2001; Haizhou and Chong, 2009), where C4.5 is used for generating the initial population of trees. Kretowski and Grzes (2006a, 2007a,b) also implemented this approach but instead of using a well-established split measure such as the gain ratio or the gini index, they opted for a dipolar split measure. 
Basgalupp et al. (2009a,b) also proposed a task-dependent knowledge based initialization for their EA for decision-tree induction. It generates ten 2-level decision trees for each data set attribute, where each one of these ten trees per attribute will possibly have different split values, since they are calculated using the information gain measure upon a different subsample of the training set. After the generation of all 2-level decision trees, they are combined according to the growing method previously described.

\section{Fitness Evaluation Methods}

In decision-tree induction, it is often desirable to maximize both the predictive accuracy and the comprehensibility of the induced tree (Freitas, 2004). EAs seem to be a natural choice for this task, since they naturally allow the evaluation of a candidate solution by simultaneously considering different quality criteria. This is not so easily performed in other data mining paradigms (Freitas, 2008). Evolutionary decision-tree induction algorithms can be roughly divided into two threads regarding fitness evaluation: (i) single-objective optimisation and (ii) multi-objective optimisation.

EAs in (i) use a single measure to guide the search for near-optimal solutions. The most common measure for evaluating individuals in evolutionary decision-tree induction is classification accuracy (or its complement, classification error):

$$
a c c=\frac{c}{m}
$$

where $c$ is the number of correctly classified instances and $m$ is the total number of instances. An accuracy-based fitness function is used in (Bandar et al., 1999; Folino et al., 2000; Fu et al., 2003a,b; Fu and Mae, 2001; Koza, 1991; Ma and Wang, 2009).

In (Llora and Garrell, 2001; Llorà and Wilson, 2004), the authors propose using $a c c^{2}$ in the fitness function, because it provides "a non- linear bias toward correctly classifying instances in decision tree $T$ while providing differential reward for imperfect decision trees". Folino et al. $(2001,2002)$ propose the use of the J-Measure, which is used to measure the quality of the disjunction of rules (paths from the root to the leaf) that describe each class. More specifically, for a $k$-class problem, there are $k$ rules of the kind (if $P_{i}$ then $y_{i}$ ), where $P_{i}$ is a set of disjunctions among paths that are used to label instances as belonging to class $y_{i}$. The J-Measure can be thus defined as

$$
J=\sum_{i=1}^{k} p\left(P_{i}\right) p\left(y_{i} \mid P_{i}\right) \log \left(\frac{p\left(y_{i} \mid P_{i}\right)}{p_{\bullet}, y_{i}}\right)
$$

where $p\left(P_{i}\right)$ is the fraction of instances satisfying condition $P_{i}, p_{\bullet, y_{i}}$ is the fraction of instances belonging to class $y_{i}, p\left(y_{i} \mid Y_{i}\right)$ is the fraction of instances that both satisfy $P_{i}$ and belong to class $y_{i}$ divided by the fraction of instances satisfying $P_{i}$, and $k$ is the total number of classes. The higher the J-Measure value, the higher the tree's predictive accuracy. 
Aitkenhead (2008) proposes a distance score for evaluating individuals which is a measure of how close a decision tree came to the correct classification. We assume that this score can only be applied to problems where the class attribute is ordinal and is converted to sequential integers that preserve the order among values. The distance score is given by

$$
D_{\text {score }}=\frac{1}{N_{x}} \sum_{i=1}^{N_{x}} 1-\left(\frac{y\left(x^{i}\right)-y^{\prime}\left(x^{i}\right)}{y_{\max }-y_{\min }}\right)^{2}
$$

where $N_{x}$ is the number of instances, $y\left(x^{i}\right)$ is the actual class value for the $i^{t h}$ instance, $y^{\prime}\left(x^{i}\right)$ is the predicted value for the same instance and $y_{\max }\left(y_{\min }\right)$ is the maximum (minimum) value of the class attribute (thus the necessity of converting categories into integers).

Kennedy et al. (1997) use the number of decisions necessary to classify all the members of the instance set as a fitness measure to be minimized. Finally, Fu et al. (2006) propose a bilinear loss function that, according to a given parameter, selects one of the possible percentiles of the classification accuracy distribution to estimate tree accuracy.

Research using a single-objective fitness function usually do not defend this choice for decision-tree induction against a multi-objective strategy. A small number of studies, however, argue that a multi-objective approach that seeks a compromise between predictive accuracy and solution complexity (tree size) as a form of parsimony pressure is not as beneficial as it may sound. Ma and Wang (2009), for instance, argue that this compromise reduces the search space and leads to a slower overall increase in accuracy. Moreover, they reckon that the search may get stuck in regions containing less accurate trees of the same size as those produced without using the complexity penalty in the multi-objective fitness function, and that, as a result, the parsimony pressure would be a downside instead of an advantage.

Research on (ii), on the other hand, argue that the balance between accuracy and parsimony is very important for efficient evolutionary search. The argument is centered on the fact that the use of accuracy alone may result in an arbitrarily complex classifier that fits the noise within the data set. Such a classifier would have high accuracy on the training set but would likely perform poorly on previously unseen data. Based on this assumption, most EAs try to optimise both predictive accuracy and simplicity (which is usually assumed to be inversely proportional to the number of nodes in a decision tree). Others seek a compromise between distinct measures, such as sensitivity and specificity in cost-sensitive approaches, which also demands the application of a multi-objective optimisation strategy.

Three general approaches are used for coping with multi-objective optimisation in evolutionary decision-tree induction: (a) weighted-formula; (b) Pareto dominance; and (c) lexicographic analysis.

Research on (a) are by far the most common. A weight (typically a user-defined parameter) is assigned to each objective (measure) to be optimised, according to its importance within the application domain. Next, these weighted objectives are summed or multiplied accordingly, 
reducing multiple objectives into a single objective. A typical strategy is to combine accuracy $(\operatorname{acc}(I))$ and tree size $(\operatorname{size}(I))$, as follows:

$$
f(I)=\alpha \times \operatorname{acc}(I)-\beta \times \operatorname{size}(I)
$$

where $\alpha$ and $\beta$ are weights and the formula has to be maximized. This approach or minor variations of it are found in (Johansson and Niklasson, 2009; Papagelis and Kalles, 2000, 2001; Podgorelec and Kokol, 1999; Rouwhorst and Engelbrecht, 2000).

Tsakonas and Dounias (2002) also propose a weighted-formula that combines accuracy and a simplicity component, though the idea is to penalize for smaller-sized trees due to domain constraints. Kretowski and Grzes (2006b) propose a small variation of (4.4) where the complexity component takes into account the number of leaves and the number of features associated to each test in non-terminal nodes (for the case of oblique decision trees). In (Kretowski and Grzes, 2007a,b), the authors propose a cost-sensitive approach that replaces accuracy in (4.4) by the misclassification cost, which is summed to the tree size in an equation whose value should be minimized. Other similar cost-sensitive approaches, which usually consider measures like sensitivity and specificity, are reported in (Hleb Babič et al., 2000; Kretowski and Grzes, 2006a; Li et al., 2005; Podgorelec and Kokol, 2001a,b; Sprogar et al., 2001; Zorman et al., 2001).

Haizhou and Chong (2009) introduce a weighted-formula that combines classification error, tree depth and number of attributes used in each path of the tree. Similarly, Reynolds and Al-Shehri (1998) propose a fitness function based on accuracy, number of nodes, number of attributes used and homogeneity of each partition. Nikolaev and Slavov (1997) offer a variation of Quinlan's pruning strategy presented in (Quinlan, 1995) as a stochastic fitness function, and perform a detailed analysis of the fitness landscape structure.

DeLisle and Dixon (2004) propose the evaluation of accuracy and complexity of decision trees by using minimum description length (MDL) as fitness function, defined as follows:

$$
\begin{aligned}
M D L & =\text { error }_{C L}+\text { tree }_{C L} \\
\operatorname{error}_{C L} & =\sum_{l \in \lambda_{T}} \log _{2}\left(\begin{array}{c}
N_{x_{l}} \\
e_{l}
\end{array}\right) \\
\operatorname{tree}_{C L} & =\left(\left|\zeta_{T}\right|+\left|\lambda_{T}\right|\right)+\left|\zeta_{T}\right| \log _{2} s+\left|\lambda_{T}\right| \log _{2} k
\end{aligned}
$$

where $N_{x_{l}}$ is the number of instances in leaf node $l, e_{l}$ is the number of misclassified instances in leaf node $l,\left|\zeta_{T}\right|$ is the total number of internal nodes, $\left|\lambda_{T}\right|$ is the total number of terminal nodes (leaves), $s$ is the total number of splits and $k$ the number of classes.

The error coding length $\left(\right.$ error $\left._{C L}\right)$ is based upon the binomial distribution and represents the number of possible combinations given the total number of observations $\left(N_{x}\right)$ and the number of incorrectly predicted observations $(e)$. This relates to the likelihood of a particular $\left(N_{x}, e\right)$ 
combination arising by random chance, and this value should be minimized. The tree coding length $\left(\right.$ tree $\left._{C L}\right)$ is dependent upon the overall size of the decision tree and should also be minimized. The authors of this particular application state that only the number of nodes and the number of leaves were actually used in the tree coding length component, since the removal of the remaining terms resulted in no alterations in performance apart from a reduced computational cost.

For the evaluation of each rule extracted from the evolved decision tree, Garcia-Almanza and Tsang (2006) propose a fitness function based on recall and a slightly variation of precision that severely penalizes the false positive cases. Since no complexity penalty is proposed, the authors suggest a pruning method to simplify the evolved decision trees.

A smaller number of papers investigate the use of the two other multi-objective strategies (namely Pareto dominance and lexicographic analysis). In one of these studies, Kim (2004) and Zhao (2007) implement a Pareto dominance approach that, instead of providing a single optimal solution based on the weighted combination of objectives, provides an optimal set of non-dominated solutions.

The Pareto multi-objective strategy proposed by Kim (2004) tries to minimize two objectives: classification error rate and tree size (measured by the number of decision rules). For ranking the individuals and discovering the Pareto optimal set, Kim implements a dominating rank method (Fayyad, 1992), where the rank of a given solution in a Pareto distribution is given by the number of elements dominating that solution. Hence, the highest possible rank is zero, for an element that has no dominator. The Pareto optimal set is given by all elements whose rank is zero. Each non-dominated solution in a discrete space of tree size represents the minimized error fitness for each number of decision rules.

Zhao (2007) also proposes a Pareto dominance approach for minimizing two conflicting objectives (e.g., false negative rate vs. false positive rate). This approach allows the decision maker to specify partial preferences on the two conflicting objectives in order to further reduce the number of alternative solutions. This tradeoff can be similarly adopted on other pairs of performance measures, such as sensitivity vs. specificity or recall vs. precision, which have been typically employed in domains such as medical diagnosis and information retrieval.

Eggermont et al. $(2003,2004 b)$ propose a lexicographic fitness ${ }^{1}$ whose highest-priority measure is misclassification error, followed by tree size. In that work, there are no tolerance thresholds, i.e., only in cases where the misclassification error of two individuals is exactly the same the tree size measure will be used to choose the best individual. Shirasaka et al. (1998); Tanigawa and Zhao (2000); Zhao and Shirasaka (1999) also propose a lexicographic fitness ${ }^{2}$ that evaluates accuracy (highest-priority measure) and tree size (total number of tree nodes). Again, there are no tolerance thresholds.

\footnotetext{
${ }^{1}$ In both references ((Eggermont et al., 2003, 2004b)), the authors refer to the lexicographic analysis as "multi-layer fitness function".

${ }^{2}$ No mention of the term "lexicographic analysis" is made throughout the three references ((Shirasaka et al., 1998; Tanigawa and Zhao, 2000; Zhao and Shirasaka, 1999)).
} 
Basgalupp et al. (2009a,b) propose LEGAL-Tree (Lexicographic Genetic Algorithm for decision-Tree induction), a GA whose fitness function implements the lexicographic approach. Once again, the objectives that will guide the search for the best individual are accuracy and tree size. The authors use both validation-set and training-set accuracy (highest and second highest priority measures, respectively), aiming to avoiding both overfitting (validation-set) and underfitting (training-set). The last objective in the priority rank is tree size, measured by the total number of tree nodes.

For a critical review of the pros and cons of each multi-objective strategy discussed in this section, see (Freitas, 2004).

\section{Selection}

Selection is the operator that chooses which individuals will undergo crossover and mutation. It is usually performed with a bias towards higher fitness in the belief that good solutions have higher potential of generating better individuals for the next generation. Some well-known selection methods are: (i) tournament selection; (ii) roulette wheel selection; and (iii) rank-based selection.

In evolutionary induction of decision trees, the most frequently used approach for selection is tournament selection, and it works as follows. A predefined number of individuals (known as tournament size) is drawn from the population, and the fittest of them is chosen to be a part of the reproducers pool. This procedure is repeated until the pool of reproducers is full. This selection method is used in (Basgalupp et al., 2009a,b; DeLisle and Dixon, 2004; Eggermont et al., 2003, 2004a,b; Garcia-Almanza and Tsang, 2006; Kennedy et al., 1997; Kim, 2004; Li et al., 2005; Ma and Wang, 2009; Niimi and Tazaki, 2000; Reynolds and Al-Shehri, 1998; Rouwhorst and Engelbrecht, 2000; Tsakonas and Dounias, 2002; Zhao, 2007).

Another popular choice in evolutionary decision trees is the roulette wheel selection, also known as stochastic sampling with replacement. This strategy is analogous to the use of a casino's roulette wheel, with each slice of the wheel proportional in size to the fitness of an individual. As a result, the probability of a individual being chosen is proportional to its fitness. Research that implement a roulette wheel selection for evolving decision trees are (DeLisle and Dixon, 2004; Folino et al., 1999, 2000, 2001, 2002; Fu et al., 2003a, 2006, 2003b; Johansson and Niklasson, 2009; Kennedy et al., 1997; Llora and Garrell, 2001; Llorà and Wilson, 2004; Nikolaev and Slavov, 1997; Oka and Zhao, 2000; Shirasaka et al., 1998; Tanigawa and Zhao, 2000; Zhao and Shirasaka, 1999).

A less-common selection method in evolutionary decision trees is rank-based selection. In decision-tree induction, rank-based selection is used in (Hleb Babič et al., 2000; Kretowski and Grzes, 2007a,b; Podgorelec and Kokol, 1999, 2001a,b; Zorman et al., 2001).

\section{Genetic Operators}

Two operators normally used to evolve a population of individuals are crossover and mutation. 
In evolutionary decision trees, crossover is usually performed in two different ways (with small variations) according to the individual representation. For fixed-length binary string encoding, it is a common approach to perform the well-known 1-point crossover. Each parent binary string selected to reproduce is split into two (in a predefined position, the "1-point") and the parents generate two new offspring by concatenating the substrings before and after this position from different parents. This crossover operator is used in (Bandar et al., 1999; Cha and Tappert, 2009; Haizhou and Chong, 2009; Kennedy et al., 1997; Smith, 2008).

Nonetheless, the vast majority of EAs encode decision trees in a tree representation, and as a result implement the standard GP crossover. This crossover selects nodes in two individuals and exchanges the entire subtrees corresponding to each selected node, generating two offspring. This operator is used in (Basgalupp et al., 2009a,b; DeLisle and Dixon, 2004; Eggermont et al., 2003, 2004a,b; Folino et al., 1999, 2000, 2001, 2002; Fu et al., 2003a, 2006, 2003b; Fu and Mae, 2001; Haruyama and Zhao, 2002; Hleb Babič et al., 2000; Kim, 2004; Kretowski and Grzes, 2006a; Llora and Garrell, 2001; Llorà and Wilson, 2004; Ma and Wang, 2009; Niimi and Tazaki, 2000; Nikolaev and Slavov, 1997; Oka and Zhao, 2000; Papagelis and Kalles, 2000, 2001; Podgorelec and Kokol, 2001a,b; Rouwhorst and Engelbrecht, 2000; Shirasaka et al., 1998; Sorensen and Janssens, 2003; Sprogar et al., 2001; Tanigawa and Zhao, 2000; Zhao, 2007; Zhao and Shirasaka, 1999; Zorman et al., 2001). Two small variations of this strategy are found in the literature. In (Podgorelec and Kokol, 1999) the authors add the constraint that selected nodes from the two parents must represent a test over the same data set attribute in order to be exchanged. Kretowski and Grzes (2006b, 2007a,b) introduce a "test-only exchange crossover", in which instead of replacing the entire subtrees, only the test represented by an attribute-value pair is replaced. This type of crossover demands the number of outcomes of the selected nodes to be the same in order to preserve the original tree structure.

EAs for induction of decision trees usually implement more than one mutation strategy. Recalling that most such EAs deal with tree-based encoding, two strategies are most used: (i) replacing a subtree by a randomly generated one; and (ii) replacing information regarding the test corresponding to the selected node.

In (i), a randomly selected subtree is replaced by a randomly generated one. Usually no further details are given on how the new subtree is randomly generated. This approach is used in (DeLisle and Dixon, 2004; Eggermont et al., 2003, 2004a,b; Folino et al., 1999, 2000, 2001, 2002; Hleb Babič et al., 2000; Kim, 2004; Ma and Wang, 2009; Niimi and Tazaki, 2000; Nikolaev and Slavov, 1997; Sprogar et al., 2001; Zhao, 2007). In a more restricted version of this approach a subtree is replaced by a random leaf node or a leaf node is replaced by a random subtree (Basgalupp et al., 2009a,b; Kim, 2004; Kretowski and Grzes, 2006a,b, 2007a,b; Nikolaev and Slavov, 1997; Podgorelec and Kokol, 2001a,b; Zorman et al., 2001).

In (ii), instead of replacing subtrees (a structural mutation), a test-based modification (a semantical mutation) is performed. EAs implementing this strategy usually allow the replacement of either the attribute, the corresponding test value or both (DeLisle and Dixon, 2004; Haruyama 
and Zhao, 2002; Hleb Babič et al., 2000; Kim, 2004; Kretowski and Grzes, 2006a,b, 2007a,b; Llora and Garrell, 2001; Llorà and Wilson, 2004; Ma and Wang, 2009; Oka and Zhao, 2000; Podgorelec and Kokol, 2001a,b; Rouwhorst and Engelbrecht, 2000; Shirasaka et al., 1998; Tanigawa and Zhao, 2000; Zhao and Shirasaka, 1999; Zorman et al., 2001). In (Papagelis and Kalles, 2000, 2001; Podgorelec and Kokol, 1999), this strategy is restricted by allowing mutation of the test-value only.

A few alternative mutation strategies are as follows. Fu et al. (2003a, 2006, 2003b); Fu and Mae (2001) propose a "self-contained" mutation strategy, in which a randomly selected (non-)terminal node is replaced by another (non-)terminal node already present in the tree, so there is no need for randomly generating a new subtree during mutation, saving processing time. Sorensen and Janssens (2003) propose two types of mutation: switch and translocation. The first switches children from the same parent and the second exchanges children from different parents in the same tree level. Both can be seen as special cases of self-contained mutation, since nodes are replaced by nodes already present in a tree. Rouwhorst and Engelbrecht (2000) propose a relational mutation, which modify the test operator corresponding to the randomly selected node. This strategy allows multiple types of operators, not only the traditional $\leq$ and $>$. For instance, suppose a node contains the test $x>5$. A relational mutation over this node could replace the operator $>$ by $\neq$, resulting in $x \neq 5$.

In fixed-length strings (Cha and Tappert, 2009; Kennedy et al., 1997), mutation is performed by simply altering a randomly chosen value in the string, which may change the attribute being used, its test value or both, depending on the approach. Hence, mutation in fixed-length strings is mainly semantical, i.e., it does not affect the decision tree structure. Nevertheless, in cases where the node type (non-terminal or terminal) is also encoded in the gene/chromosome, it is possible that the structure of the decision tree is modified by mutation.

\section{Parameter Setting}

The parameter values of an EA can largely influence whether the algorithm will find a near-optimum solution, and whether it will find such a solution efficiently. Choosing correctly the parameters, however, is a time-consuming task and considerable effort has been dedicated to the development of good heuristics able to overcome this problem (Michalewicz and Schmidt, 2007). Espejo et al. (2010) state that the large number of parameters that must be defined in order to have a working system is in fact one of the pitfalls of GP-based classifiers (an argument easily generalisable for other EAs).

The most common parameters in EAs for decision-tree induction are population size, number of generations, probabilities of application of different genetic operators and maximum size of decision trees at initialization or during the evolutionary process. In practice, several preliminary runs are usually required in order to tune these parameters. In some studies, parameters are fine-tuned to suit each data set employed in the experiments (Ma and Wang, 2009; Rouwhorst 
and Engelbrecht, 2000). However, most authors prefer to present a set of default parameter values followed by a sentence like "parameter values were empirically defined". We believe this is due to the fact that no work has exhaustively investigated the influence of different parameter settings in evolutionary decision-tree induction, like De Jong and Spears have for function optimisation (Jong and Spears, 1991). A possible explanation is that decision-tree induction is data dependent, which means different classification data sets may present very distinct error surfaces, favoring particular sets of parameter values.

The population size parameter influences the number of candidate solutions evaluated by the EA. Some values for this parameter used in the literature are 50 individuals in (Bandar et al., 1999; Nikolaev and Slavov, 1997). 100 individuals in (Eggermont et al., 2003, 2004a,b; Fu and Mae, 2001; Rouwhorst and Engelbrecht, 2000), 200 individuals in (Papagelis and Kalles, 2000, 2001; Shirasaka et al., 1998; Tanigawa and Zhao, 2000; Zhao and Shirasaka, 1999), 400 individuals in (Folino et al., 2000, 2001, 2002), 500 individuals in (Basgalupp et al., 2009a,b; Kim, 2004; Niimi and Tazaki, 2000) and 1000 individuals in (DeLisle and Dixon, 2004; Garcia-Almanza and Tsang, 2006).

Another parameter, the number of generations, is usually the EA's stopping criterion. A popular choice in EAs for inducing decision trees is evolving individuals for 1000 generations (DeLisle and Dixon, 2004; Kim, 2004; Kretowski and Grzes, 2007a,b; Podgorelec and Kokol, 2001b). However, in some EAs the number of generations is as small as 10 (Fu et al., 2003b), 30 (Li et al., 2005) or 50 (Bandar et al., 1999; Fu et al., 2003a, 2006; Fu and Mae, 2001), whilts in other EAs it is as large as 5000 (Tanigawa and Zhao, 2000; Zhao and Shirasaka, 1999) or 10000 (Kretowski and Grzes, 2006b; Zhao, 2007). A useful strategy is to set a secondary stopping criterion to identifying cases of fast convergence. For instance, if the EA does not improve the best individual during a pre-determined number of generations, it can be stopped, being assumed that it has already converged to the (near) optimal solution. Once again, there is no consensus on the most suitable value for this parameter. Values like 3\% (Basgalupp et al., 2009a,b), 10\% (Kretowski and Grzes, 2006b), 20\% (Kretowski and Grzes, 2007a,b) and 25\% (Johansson and Niklasson, 2009; Papagelis and Kalles, 2000) of the number of generations have been used.

Crossover is the main genetic operator for evolving individuals. Most EAs use a high probability of crossover, such as 60\% (Bandar et al., 1999; Kim, 2004; Tsakonas and Dounias, 2002), 80\% (Garcia-Almanza and Tsang, 2006; Johansson and Niklasson, 2009; Niimi and Tazaki, 2000; Nikolaev and Slavov, 1997; Rouwhorst and Engelbrecht, 2000) or 90\% (Basgalupp et al., 2009a,b; Eggermont et al., 2003, 2004a,b; Folino et al., 2000; Li et al., 2005; Papagelis and Kalles, 2000, 2001). Mutation, on the other hand, usually occurs much less often. The most common value for mutation rate is 1\% (Folino et al., 2000; Fu et al., 2003a,a, 2006; Fu and Mae, 2001; Johansson and Niklasson, 2009; Li et al., 2005; Shirasaka et al., 1998; Tanigawa and Zhao, 2000; Zhao and Shirasaka, 1999). Other popular values range from 2 to 10\% (Bandar et al., 1999; Basgalupp et al., 2009a,b; Garcia-Almanza and Tsang, 2006; Kim, 2004; Niimi and Tazaki, 2000; Papagelis and Kalles, 2000, 2001; Podgorelec and Kokol, 2001b; Zhao, 2007). 
In the popular tournament selection method, the size of the tournament is yet another parameter that needs to be set. Blickle and Thiele (1996) claim that typical values for tournament size are 2 and 3, and that there is a great loss of diversity when the value is higher than 5. However, there is no clear consensus on tournament size in evolutionary decision tree research. Some papers seem to follow the suggestion of Blickle and Thiele by setting a tournament size of up to 5 individuals (Eggermont et al., 2003, 2004a,b; Garcia-Almanza and Tsang, 2006; Kim, 2004; Li et al., 2005). Others choose arbitrary values such as 6 (Tsakonas and Dounias, 2002), 7 (Zhao, 2007), 8 (Ma and Wang, 2009), 10 (Rouwhorst and Engelbrecht, 2000), 30 (Basgalupp et al., 2009a,b), 80 (Tanigawa and Zhao, 2000; Zhao and Shirasaka, 1999) or 100 (Shirasaka et al., 1998). None of them make a detailed analysis on the effects of different tournament sizes.

Most EAs for decision-tree induction are generational, i.e., individuals of the current population are replaced by their offspring at each generation (iteration). In order to avoid losing good solutions in this process, it is a common practice to keep the best individual(s) from the current generation to the next, which is named elitism. The number of individuals to be kept is yet another parameter that needs to be set. A popular choice is a small number of individuals to be part of the elite (1 to 5 individuals) (Garcia-Almanza and Tsang, 2006; Ma and Wang, 2009; Podgorelec and Kokol, 2001b).

Finally, most EAs use a maximum tree size at initialization or during the evolutionary process. This maximum size is usually defined in terms of tree depth. Trees are usually size-limited during initialization in order to speed-up the algorithms' running times. Typical initialization size limits are 3 levels (Basgalupp et al., 2009a,b), 4 levels (Folino et al., 2000, 2001, 2002; Li et al., 2005), 7 levels (Johansson and Niklasson, 2009) and 10 levels (Zhao, 2007). Some EAs also limit the tree depth during evolution; for instance, with a limit of 17 levels in (Li et al., 2005) or 30 levels in (Zhao, 2007) in 30 levels.

Note that parameter setting in EAs is itself a research field and it goes way beyond trial and error analysis. However, none of the EAs for decision-tree induction reviewed so far make a detailed analysis on parameter values. Ma and Wang (2009) suggest a heuristic method to set some EA parameters. The idea is to use a neural network a priori of the EA execution. For instance, the maximum number of generations can be defined as the maximum number of iterations a feed forward neural network needs in order to find an acceptable result. They also state that setting parameters in an EA is no more difficult than choosing the numbers of layers and hidden neurons in feed forward neural networks.

\subsubsection{Oblique Decision Trees}

Oblique decision trees, also referred to as (non-) linear decision trees, are a common alternative to the traditional axis-parallel approach. Oblique decision trees are usually much smaller and often more accurate than axis-parallel decision trees, though at the expense of more computational effort and loss of comprehensibility. 
Several approaches based on EAs have been proposed to evolve oblique decision trees. Bot and Langdon $(2004,2000)$ propose a GP for evolving oblique decision trees. In this work, each GP individual is encoded as a tree with function nodes and terminals. A function node has as its children a tuple $\left(\left\{w_{i}, a_{i}(x)\right\}\right.$, threshold, ifTrue, if False), where $w_{i}$ and $a_{i}(x)$ are the $i^{\text {th }}$ weight constant and attribute pair, respectively. Depending on the function node type, there can be $n$ pairs $\left\{w_{i}, a_{i}(x)\right\}$. Still in this tuple, threshold is a constant terminal and ifTrue (ifFalse) can be either classification terminals or other function nodes. Terminals are either constants (doubles), variables (integers) or classifications (integers). Nodes are evaluated as follows:

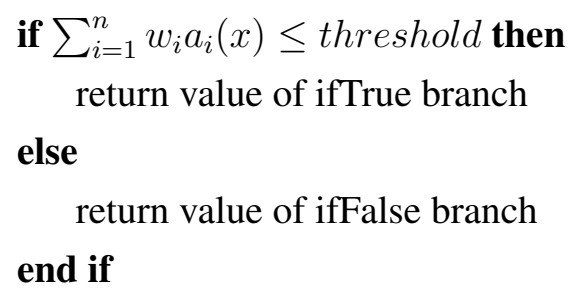

This GP is strongly typed (Montana, 1995), i.e., the data types of functions and terminals are specified to ensure that only valid individuals are generated. A terminal of variable type is an integer ranging from 0 to the number of data set attributes -1 (an attribute index). A terminal of constant type is a floating-point within a predefined range. A terminal of classification type is an integer ranging from 0 to the number of classes -1 (class index). The function set allows up to 3 attributes to be tested within each node of the decision tree.

This GP has the following parameters. Individuals are selected by tournament selection with size 7. A population of 250 individuals evolve during 1000 generations. The initial population is created through the ramped half and half method (Koza, 1992a,b). Crossover and mutation rates were empirically set to 0.5 each. The fitness function is a weighted-formula that includes validation set accuracy and a penalty factor to lower the fitness value, multiplied by either the tree depth or the total number of nodes. The penalty factor is used to alleviate the bloat problem (Banzhaf et al., 1998).

In (Bot et al., 2000), Bot expanded his work from (Bot and Langdon, 2000) by introducing limited error fitness (LEF) (Gathercole, 1998), Pareto scoring and fitness sharing (Goldberg, 1989). LEF is a technique for speeding up fitness calculation in supervised learning problems where each individual is evaluated on a number of training cases. If the error score achieved by the individual in these training cases is above a given threshold, all the remaining training cases are counted as errors. This is to prevent poorly-designed individuals to be tested over the entire training set, wasting computational time. Individuals whose number of errors is below the given threshold are evaluated over the entire training set.

Pareto scoring and fitness sharing are also introduced. Since the use of Pareto scoring leads to populations that tend to converge to a few (and possibly suboptimal) solutions (Goldberg, 1989), fitness sharing is used to ensure that more different local minima are found. It is a niching technique 


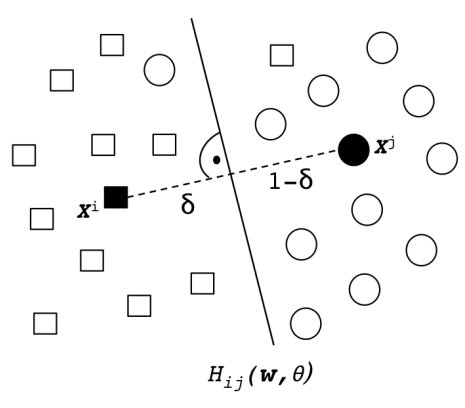

Figure 4.4: Initializing a hyperplane with randomly selected mixed dipole. Adapted from (Kretowski and Grzes, 2005).

that avoids premature convergence by penalizing solutions with many "neighbors", i.e.individuals very similar to others. For such, a sharing function $s(i)=\sum_{j} s(i, j)$ is calculated for each individual $i$ in the Pareto front. The distance (similarity) of individual $i$ to every other individual is computed and, the more similar an individual $j$ is to $i$, the higher the value of $s(i, j)$ and thus of $s(i)$. Finally, the individual of the Pareto front with the lowest value of $s($.$) is selected to reproduce.$

Another work, by Kretowski and Grzes (2005), introduced GEA-ODT (Global Evolutionary Algorithm for Oblique Decision-Tree induction). GEA-ODT encodes each decision tree as a tree with splitting hyperplanes in non-terminal nodes and class labels in the leaves. Each hyperplane is represented by a $(n+1)$-dimensional table, accounting for $\mathbf{w}$ and $\theta$. The population of individuals is initialized by generating each individual through a top-down algorithm that searches at each node for the best hyperplane. Hyperplanes are randomly generated based on randomly chosen mixed dipole $\left(\mathrm{x}^{\mathbf{i}}, \mathrm{x}^{\mathbf{j}}\right)$. A dipole (Bobrowski, 1996) is a pair of instances $\left(\mathrm{x}^{\mathbf{i}}, \mathrm{x}^{\mathbf{j}}\right)$, and it is referred as a mixed dipole if and only if its instances belong to different classes. Thus, an hyperplane $H(\mathbf{w}, \theta)$ splits the dipole $\left(\mathrm{x}^{\mathbf{i}}, \mathrm{x}^{\mathrm{j}}\right)$ if and only if:

$$
\left(\mathbf{w}^{\mathbf{T}} \mathbf{x}^{\mathbf{i}}-\theta\right) \times\left(\mathbf{w}^{\mathbf{T}} \mathbf{x}^{\mathbf{j}}-\theta\right)<0
$$

which means that $\mathrm{x}^{\mathrm{i}}$ and $\mathrm{x}^{\mathrm{j}}$ are on opposite sides of the dividing hyperplane.

For generating random hyperplanes, a dipole $\left(\mathbf{x}^{\mathbf{i}}, \mathbf{x}^{\mathbf{j}}\right)$ is randomly selected and the $H_{i j}(\mathbf{w}, \theta)$ hyperplane is generated by setting $\mathbf{w}$ and $\theta$ as:

$$
\begin{aligned}
\mathbf{w} & =\mathbf{x}^{\mathbf{i}}-\mathbf{x}^{\mathbf{j}} \\
\theta & =\delta\left(\mathbf{w}^{\mathbf{T}} \mathbf{x}^{\mathbf{i}}\right)+(1-\delta)\left(\mathbf{w}^{\mathbf{T}} \mathbf{x}^{\mathbf{j}}\right)
\end{aligned}
$$

where $\delta \in\{0,1\}$ is a random coefficient that scales the distance to the opposite ends of the dipole. Figure 4.4 presents this rationale.

The fitness function in GEA-ODT is a weighted-formula that penalizes model complexity (tree size). It is given by

$$
f(I)=Q(I)-(\alpha \times \operatorname{size}(I))
$$


where $Q(I)$ is the quality measure over the training set (we assume accuracy is a suitable quality measure for the purpose of maximizing $f(I)$ ), and size $(I)$ is the size of individual $I$ (total number of nodes). The parameter $\alpha$ scales the importance of the penalty term.

In GEA-ODT, mutation occurs with a $1 \%$ probability. It can either alter the node role or its corresponding hyperplane. More specifically, a non-terminal node can be pruned to a leaf or have its hyperplane modified. A hyperplane can be modified by either standard mutation (perturbation of the weights that form the hyperplane) or by the dipole operator. The dipole operator is a task-dependent knowledge based operator that seeks to shift the hyperplane in order to divide mixed dipoles or unite pure dipoles. Finally, when dealing with a leaf node, the mutation operator can swap this node for a new non-terminal node. Standard one-point crossover in trees is performed. Selection is performed through linear ranking with elitism.

\subsection{Regression}

Decision trees for regression are an effective approach for predicting continuous values while enabling the analysis of the variables that led to that particular prediction. They are usually regarded as either regression trees or model trees, according to the content of their leaf nodes. We review EAs for inducing regression or model trees next.

\subsubsection{Regression Trees}

A regression tree is a special type of decision tree where the target attribute is continuous. Thus, each leaf node of the regression tree holds a continuous value instead of a class label. This continuous value is the average value for the target attribute of all instances that reach that particular leaf node.

TARGET (Tree Analysis with Randomly Generated and Evolved Trees) (Fan and Gray, 2005; Gray and Fan, 2008) is an EA that evolves regression trees where each candidate solution is an axis-parallel regression tree of variable size and shape. The initial population consists of 25 randomly created trees. The authors report that experiments performed with forest sizes between 10 and 100 trees, showed no apparent impact on the outcome. The random generation of trees starts with a single root node, that has a probability $p_{\text {split }}$ of becoming a split. Otherwise, it will be a leaf. If the node bocomes a split, an attribute from the data set and a split value are randomly chosen from a pool of candidate attributes and split values and two child nodes are created. This procedure is repeated until no more nodes can be split.

For evaluating the trees, TARGET measures their fitness by using the Bayesian information criterion (BIC), a statistical model selection criterion based on likelihood (Schwarz, 1978). It is a weighted-formula that penalizes model complexity (size of the tree). It is expressed as

$$
B I C=-\frac{m}{2} \ln 2 \pi-\frac{m}{2} \ln \frac{S S E}{m}-\frac{m}{2}-\frac{1}{2} p \log m
$$


where $p$ is the effective number of parameters in the tree model, $m$ is the size of the training set and $S S E$ is the residual sum of squares. The last term in (4.12) is the model complexity penalty, which is a function of both the effective number of parameters $p$ and the training sample size $m$.

One critical problem of using the BIC criterion in TARGET is determining the value of $p$, an essential part of the model complexity penalty term. Large values of $p$ may lead to smaller trees with less predictive performance. The authors defined $p=n t+1$ to account for estimating the constant error variance term and a mean parameter within each of the $n t$ terminal nodes. Even though they acknowledge the fact that the effective number of parameters estimated is actually much higher than $n t+1$, due to split rule selections made during the tree construction process, they state that further research is required to determine the appropriate adjustment of the model complexity penalty term.

Kretowski and Czajkowski (2010) propose an EA for regression tree induction called GRT (Global induction of Regression Trees). Each candidate solution is an axis-parallel regression tree. The trees in the initial population are initialized through traditional top-down regression tree algorithms such as M5 (Quinlan, 1992; Wang and Witten, 1997) and CART (Breiman et al., 1984) on random subsamples from the original data set. The recursive partitioning of the initial trees stops when: (i) all training objects in a node have the same predicted value; (ii) the number of instances in a node is lower than a predefined value; or (iii) the predefined maximum tree depth is reached.

GRT's crossover operation has three variants: (i) subtrees are swapped between two individuals, with random selection of nodes; (ii) the split test between two non-terminal nodes of different individuals are swapped, though keeping their subtree structures; and (iii) branches from two randomly selected nodes in two different individuals whose subtrees have the same size are swapped.

Mutation is stochastically performed (the default probability is 0.8 ) in either non-terminal nodes or leaves. In non-terminal nodes, nodes in higher levels of the tree are mutated with a lower probability than those in lower levels. Among nodes in the same level, the absolute error is calculated for the node subtree in order to rank nodes: a higher value of absolute error increases the probability a node being mutated. The same ranking procedure is applied to leaf nodes (except for pure nodes). For non-terminal nodes, mutation can assume the following variants: (i) replacement of a split test according to a traditional split search mechanism; (ii) shifting splitting thresholds (for continuous attributes) or regrouping attribute values (for nominal attributes); (iii) replacement of a split test by a pre-existing test; (iv) replacement of a subtree by another subtree from the same node; (v) replacing a subtree by a leaf node. Mutation may transform leaf nodes into non-terminal nodes if the instances contemplated by that particular node are heterogeneous.

The selection mechanism adopted by GRT is linear ranking, and the best individual of each generation is kept to the next (elitism). The fitness function searches for a compromise between mean absolute error and tree size, as follows: 


$$
f(I)=\frac{M A E(I)}{M A E_{\max }}+\alpha(\operatorname{size}(I)-1)
$$

where $M A E(I)$ stands for the mean absolute error of individual $I$ over the training set, $M A E_{\text {max }}$ is the mean absolute error of the entire learning set, and size $(I)$ is the size of individual $I$. The parameter $\alpha$ weights the relative importance of the complexity penalty and subtracting 1 from the complexity term eliminates the penalty factor for trees that consist of a single leaf.

Hazan and Ramirez (2006); Hazan et al. (2006) propose a strongly-typed GP approach (STGP) ${ }^{3}$ for axis-parallel regression tree induction. Although they focus on inducing regression trees for modeling expressive performance (musical computation), their approach is easily generalised to any domain. In STGP function nodes can be either a test over an attribute (e.g., a "less than" operator, hereby called LT), an if-then-else construct (IF) or a constant generator of either attribute or regression values, namely EFV and ERV, respectively. The LT construct operates over two arguments of fixed types: FeatValue and RegValue, and returns a boolean value. IF operates over three arguments: a boolean, resulting from the constructor LT and two arguments of RegValue type. IF returns a RegValue value, that can be either a constant or any other construct. Terminal nodes are constants representing either attribute values or regression values (target attribute outputs), generated by either EFV or ERV functions. Figure 4.5 demonstrates a typical tree from STGP.

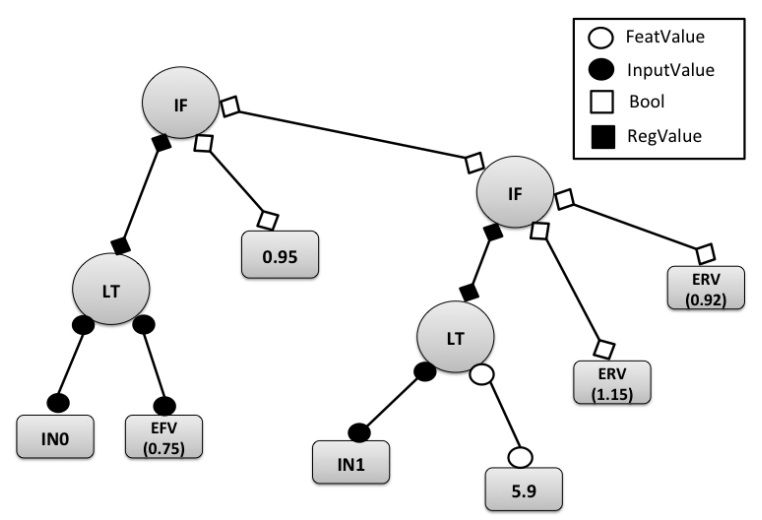

Figure 4.5: STGP regression tree example. Typed connections are presented. Adapted from (Hazan et al., 2006).

The population size of STGP is fixed in 200 individuals, and the algorithm halts when either the limit of 500 generations has been reached or the best solution fitness is $\geq 0.95$. Tournament selection is the strategy for selecting individuals to reproduce. A typical subtree swap crossover is performed with a 0.9 rate. Mutation can either swap a subtree with a newly generated one $(0.01$ rate), replace a branch by one of its children (0.05), swap subtrees from the same individual (0.1) or disturb constant values (rate not informed). A maximum depth of 10 levels is set, though the authors state that they do not search for low complexity (smaller sized) solutions.

\footnotetext{
2006).

${ }^{3}$ STGP was designed within the Open Beagle Framework for evolutionary computation (Gagné and Parizeau,
} 
STGP's fitness function is given by the inverse of RMSE (root mean squared error), $f(I)=$ $1 /(1+R M S E(I))$, where RMSE is taken over the training set and predicted duration ratio, averaged over the notes of a musical fragment, itself averaged over all the training fragments. For more conventional applications, the RMSE could be taken directly over the predicted values of the individual $I$. The authors also present domain-specific fitness functions not presented here due to space constraints.

\subsubsection{Model Trees}

Model trees are a special case of decision trees also developed for regression problems. The main difference between a model tree and a regression tree is that whereas each leaf node in a regression tree outputs a single continuous value, in model trees each leaf node holds a (non-) linear model whose output is the final prediction value.

GPMCC (Potgieter and Engelbrecht, 2008) (Genetic Programming approach for Mining Continuous-valued Classes) is a framework proposed to evolve model trees with non-linear models in their leaves. Its structure is divided into three different parts: (1) GASOPE (Potgieter and Engelbrecht, 2007), a GA that evolves polynomial expressions; (2) K-Means (Lloyd, 1982), a traditional clustering algorithm used to sample the training set; and (3) a GP to evolve the structure of model trees. Trees are generated by randomly expanding a node and randomly selecting attributes and split values. Tree growth is dictated by a parameter that indicates the maximum tree depth. The fitness function used by GPMCC is an extended form of the adjusted coefficient of determination (4.14)

$$
R_{a}^{2}=1-\frac{\sum_{i=1}^{N_{x}}\left(y\left(x^{i}\right)-y^{\prime}\left(x^{i}\right)\right)^{2}}{\sum_{i=1}^{N_{x}}\left(y\left(x^{i}\right)-\bar{y}\right)^{2}} \times \frac{N_{x}-1}{N_{x}-d}
$$

where $N_{x}$ is the size of the set, $y\left(x^{i}\right)$ is the actual output of the $i^{t h}$ instance, $y^{\prime}\left(x^{i}\right)$ is the predicted output for the same instance, $\bar{y}$ is the average output of all instances and $d$ is a complexity factor that penalizes both the size of an individual (number of nodes) and the complexity of each model of the terminal nodes (number of terms and their corresponding order). The higher the value of complexity factor $d$, the lower the value of $R_{a}^{2}$. The best individuals are those with the higher values of $R_{a}^{2}$.

One important remark is that GPMCC has a total of 42 configurable initialization parameters that control several steps of the algorithm. The authors claim that the influence of GPMCC parameter values on the performance obtained was empirically investigated and that it was shown that the performance was not significantly affected by different parameter values. Based on these results, it is not clear the real utility of so many configurable options. As a second point of criticism, GPMCC seems to be overly-complex, and the results do not seem to justify all the choices made during its development.

Barros et al. (2010, 2011c) propose an EA called E-Motion (Evolutionary MOdel Tree InductiON) for axis-parallel model tree induction, where each individual is represented as a tree 
of variable shape and size. The initialization of individuals is domain knowledge-based, as it combines single nodes whose attribute tests are dictated by the expectation of standard deviation reduction (SDR). E-Motion generates a split rule for each data set attribute that maximizes the SDR criterion. The initial trees are random combinations of these basic trees that consist of a single node (attribute + split rule).

E-Motion allows the user to choose between two types of multi-objective optimisation in the fitness function. The first one is a weighted-formula that accounts for RMSE (root mean squared error), MAE (mean absolute error) and tree size, where each measure has a user-defined weight. The second one involves a lexicographic analysis, where these three measures are ranked based on the user's priorities. In this case, user-defined thresholds may define whether the highest-priority measure is enough to select the best individual or if the subsequent measures should also be evaluated.

E-Motion implements standard one-point crossover in trees. Two different mutation strategies are used to variate individuals' sizes: a shrinking mutation, where a subtree is replaced by a leaf node, and an expanding mutation, where a leaf node is replaced by a two-level subtree. Finally, a filter is applied to guarantee consistency of the linear models at each leaf node.

\subsection{Decision Tree-Components}

All EAs reviewed so far evolve individuals which represent decision trees. In this section, we discuss EAs with a different purpose: to improve a decision tree classifier's component. Hence, they do not evolve decision trees per se, but components of decision tree classifiers. We divide such EAs into 3 groups, according to the type of component being evolved: (i) Hyperplanes; (ii) Pruning Methods; (iii) Other Components.

\subsubsection{Hyperplanes}

EAs evolving full oblique trees were reviewed in Section 4.3.2. However, some EAs evolve only a hyperplane for each node of the oblique tree. More specifically, each individual in these EAs is a combination of attributes and constants that define a hyperplane.

In (Chai, 1996; Chai et al., 1996), Chai et al. propose a binary linear decision tree approach for piecewise linear classification, named BTGA. At each non-terminal node of the tree, a GA searches for a linear decision function, optimal in the sense of maximum impurity reduction (the Gini index criterion is used). We can formalize the problem of inducing a binary linear decision tree as finding the vector $\mathbf{w}^{*}$ that maximizes the impurity reduction at node $t$, i.e.,

$$
\mathbf{w}^{*}=\arg \max _{\mathbf{w}} \Delta i\left(\mathbf{X}_{\mathbf{t}}, \mathbf{w}\right)
$$


A GA is applied to globally search the solution-space for the best possible combination of values in $\mathbf{w}$, which is $\mathbf{w}^{*}$. In this GA, w is encoded as a binary-string, such that the gene $G^{i}$ encodes the $i^{t h}$ possible value of $\mathbf{w}$ and the chromosome $G$ consists of the union of genes, i.e., $G=G^{1} \cup G^{2} \ldots \cup G^{n+1}$.

The fitness function is given by the impurity reduction (Gini index) calculated for each chromosome. The selection scheme is the roulette wheel, where chromosome $G_{j}$ is assigned a selection probability $f\left(G_{j}\right) / \sum_{i=1}^{n c} f\left(G_{i}\right)$, where $n c$ is the total number of chromosomes and $f($. is the fitness function. The selected chromosomes participate in a two-point crossover, an effective alternative to the traditional one-point crossover, since more chromosome substructures can be preserved and combined. Mutation is given by simply bit-flipping genes with a low-probability.

Palaniappan et al. (2000) extended BTGA (BTGA+) by implementing three different impurity measures (namely Gini index, information gain and twoing rule). Besides, they investigated a Bayesian initialization of the individuals (weights) with randomization process. The Bayesian initialization uses the common covariance weighted vector between the means of the dominant class, $\omega_{0}$, and the remaining node examples, $\omega_{1}$, with $\mathbf{w}=\Sigma^{-1}\left(\mu_{0}-\mu_{1}\right)$ and an independent parameter, $w_{0}$, given by:

$$
\begin{aligned}
w_{0}= & -\frac{1}{2}\left[\left(\mu_{0}^{T} \times \Sigma^{-1} \times \mu_{0}\right)-\left(\mu_{1}^{T} \times \Sigma^{-1} \times \mu_{1}\right)\right] \\
& +\ln \frac{p_{\bullet, \omega_{0}}}{p_{\bullet, \omega_{1}}}
\end{aligned}
$$

where $\Sigma^{-1}$ is the inverse covariance matrix (concentration matrix).

Kim et al. (2004a,b) propose a hybrid approach that uses a GA for selecting hyperplanes in each node of a binary oblique decision tree. In this work, the GA performs feature selection so as to reduce the classification error rate in a binary decision tree. The GA looks for the optimal linear combination of features able to divide the instances in two disjunct subsets. This process is carried out at each node of the binary tree that is being created. Thus, for each node, a linear combination of features selected by the GA divides the attribute space into two different subsets until each subset has instances belonging to a unique class.

Each individual in the GA is a $n$-dimensional linear decision function. A binary string representation encodes the linear decision function, and each string has $n^{2} \times \gamma$ bits, where $n$ is the number of dimensions and also the number of segments necessary to encode a single feature, and $\gamma$ is the length of each segment. The fitness function is given by:

$$
f(I)=\frac{1}{1+\left(w_{e} \times \text { error }\right)+\left(w_{b} \times \text { balance }\right)}
$$

and

$$
\text { balance }=\sqrt{\frac{\sum_{j=1}^{2}\left(N_{x_{j}}-\frac{N_{x}}{2}\right)^{2}}{\left(\frac{N_{x}}{2}\right)^{2}}}
$$


where $N_{x}$ is the total number of instances, $N_{x_{j}}$ is the number of instances that reach the $j^{\text {th }}$ node, error is the classification error and $w_{e}$ and $w_{b}$ are weights associated to error and balance, respectively. In these equations, balance is the balance coefficient whose values tend to decrease when the number of instances in each group become similar.

Cantu-Paz and Kamath (2000, 2003) also investigate the use of EAs for designing optimal hyperplanes. They propose two different strategies for expanding OC1 (Murthy et al., 1993) (an oblique decision-tree induction algorithm): (i) a (1+1) evolution strategy (ES) with self-adaptive mutations (named OC1-ES); and (ii) a GA with real-valued genes (named OC1-GA).

OC1-ES evolves a single individual ${ }^{4}$, a vector of real-valued coefficients, $w_{1}, w_{2}, \ldots, w_{n+1}$, for a $n$-dimensional data set. The individual is initialized with the best axis-parallel split found by OC1. For each hyperplane coefficient, there is a corresponding mutation rate, $\sigma_{1}, \sigma_{2}, \ldots, \sigma_{n+1}$, initially set to 1 . At each iteration of the evolution strategy, the mutation rates are updated and so are the coefficients, according to the following rule:

$$
\begin{aligned}
v & =N(0,1) \\
\sigma_{i}^{t+1} & =\sigma_{i}^{t} \exp \left(\tau^{\prime} v+\tau v\right) \\
w_{i}^{t+1} & =w_{i}^{t}+\sigma_{i}^{t+1} v
\end{aligned}
$$

where $N(0,1)$ is a realization of a unitary normal variate, $\tau=(\sqrt{2 \sqrt{n}})^{-1}$ and $\tau^{\prime}=(\sqrt{2 n})^{-1}$. The ES is run for 1000 iterations.

OC1-GA evolves a population of individuals represented by a real-valued set of coefficients (the same used in OC1-ES). Tournament selection without replacement selects the individuals that will be subjected to uniform crossover (probability of $100 \%$ to happen). No mutation is implemented in OC1-GA. The population size is set to $20 \sqrt{n}$, along the lines of a population-sizing

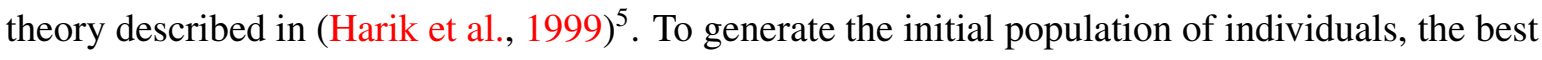
axis-parallel hyperplane is assigned to $10 \%$ of the individuals, and the remaining $90 \%$ are randomly generated in the range $[-200,200]$. The fitness is evaluated by the calculus of the twoing criterion (Breiman et al., 1984).

Kretowski (2004) proposes an EA that evolves hyperplanes for oblique decision trees. The proposed approach is based on the dipole concept (see Section 4.3.2). The oblique decision tree is built through a top-down approach, and the "optimal" decision function is selected at each tree node. For evolving decision functions, a fixed-length real-valued chromosome of size $n+1$ represents the hyperplane ( $n$ weights and $\theta$ ). For generating the initial population, a random mixed dipole $\left(\mathbf{x}^{\mathbf{i}}, \mathbf{x}^{\mathbf{j}}\right)$ is selected and the hyperplane $H_{i j}(\mathbf{w}, \theta)$, which is perpendicular to the segment that connects the opposite sides of the dipole (placed in halfway), is formed by setting $\mathbf{w}=\mathbf{x}^{\mathbf{i}}-\mathrm{x}^{\mathbf{j}}$ and $\theta=1 / 2\left[\left(\mathbf{w}^{\mathbf{T}} \mathbf{x}^{\mathbf{i}}+\mathbf{w}^{\mathbf{T}} \mathbf{x}^{\mathbf{j}}\right)\right]$. The fitness function is given by

\footnotetext{
${ }^{4}$ Note that ES, unlike GAs or GP, evolves a single individual instead of a population

${ }^{5}$ Harik et al. state that the population size required to reach a solution of a particular quality is $O(\sqrt{n})$
} 


$$
\begin{aligned}
f(x) & =f(\mathbf{w}, \theta) \times\left[(1-\beta)+\beta \frac{n^{\prime}}{n}\right], \text { where } \\
f(\mathbf{w}, \theta) & =f_{\text {mixed }}+\alpha\left(1-f_{\text {pure }}\right)
\end{aligned}
$$

where $f_{\text {mixed }}\left(f_{\text {pure }}\right)$ is the fraction of divided mixed (pure) dipoles, $\alpha$ controls the importance of pure dipoles, $\beta \in\{0,1\}$ defines the complexity of the test (in terms of number of parameters), $n^{\prime}$ is the number of non-zero weights and $n$ is the dimensionality of the problem.

Standard two-point crossover is employed and the mutation operator is slightly modified in order to increase the chances a gene can be set to 0 . Since the weights are real-valued, chances of eliminating the importance of one attribute (i.e., setting it to 0 ) are slim, so the mutation operator is modified to enhance the chances of feature selection (eliminating attributes). Additionally, a dipolar operator is implemented, as follows. First, the dipole type is drawn (mixed or pure). If the mixed type is selected, one dipole is drawn from the set of non divided mixed dipoles and the hyperplane is shifted to separate the pair of instances. The new position is obtained by modifying only one randomly chosen attribute. If it is the pure type, one dipole is drawn from the set of divided pure dipoles. The hyperplane is shifted to avoid separation of objects from the same class by once again modifying one randomly chosen weight. Selection is made through linear ranking and the best individual from a generation is kept to the next (elitism).

Shali et al. (2007) propose a GP, named GIODeT for evolving combinations of attributes for each node split. The C4.5 algorithm (Quinlan, 1993) is used for constructing the decision tree in a top-down fashion, but instead of using the typical univariate tests at each node, a GP evolves a set of relations for combining the data set attributes. The authors propose the following functions for the internal nodes of GIODeT:

- Mathematical: $+,-, \times, /, \log , \sqrt{ }$

- Relational: $\leq$

- Logical: nand, not

An expression generator component is responsible for combining these unary and binary functions with the set of nominal and numeric attributes, generating a mathematical expression with arbitrary size. Note that the GP is run once for each node of the decision tree. Tournament selection is performed on 15 randomly selected individuals in order to choose those that will undergo crossover and mutation. Standard GP crossover and mutation are applied with probabilities of 0.65 and 0.2 , respectively. The fitness function is given by

$$
f(I)=\text { GainRatio } \times\left(\text { GainRatio }+\frac{1}{\text { size }(I)}\right)
$$


where GainRatio is the standard split criterion implemented in C4.5 (Quinlan, 1993) and size(I) is a function that returns the total number of nodes of the GP individual $I$.

\subsubsection{Pruning Methods}

Pruning is an important component of decision-tree induction algorithms, because it can avoid model overfitting. Given a hypothesis space $H$, a hypothesis $h \in H$ is said to overfit the training data if there exists some alternative hypothesis $h^{\prime} \in H$ such that $h$ has a smaller error than $h^{\prime}$ over the training examples, but $h^{\prime}$ has a smaller error than $h$ over the entire distribution of instances.

Overfitting is particularly critical in decision-tree induction, since decision trees can perfectly classify the training examples. If there is noise in the training set, the induced decision tree will learn how to classify it and thus will perform poorly on unseen data. Lack of representative samples in the training data can also lead to model overfitting, because the resulting decision tree will make its classification based on small number of instances. Common approaches for avoiding overfitting are prepruning (halting the algorithm before generating a fully grown tree that perfectly fits the data) and post-pruning (grow a full decision tree and later pruning subtrees according to error estimates). Evolutionary alternatives to decision tree pruning are reviewed next.

Chen et al. (2009) propose a GA for decision tree pruning where each individual is a fixed-length linear chromosome. Each gene dictates whether a subtree should be pruned or not. More specifically, a decision tree is fully grown by a traditional decision-tree induction algorithm (ID3 (Quinlan, 1986)) and then linear chromosomes with size equal to the number of edges of the full tree are randomly generated. One-point crossover and bit-flip mutation are implemented (rates are not informed). The fitness function to be minimized is given by the sum of the total number of nodes and the error rate. Surprisingly, during fitness evaluation, the error rate is said to be calculated over the test set (a serious experimental mistake, since the test set class labels are supposed to be unknown, and can only be used to validate the best individual of the EA, and not every individual in each generation).

Shah and Sastry (1999) propose a new pruning algorithm for oblique decision trees in binary classification problems using either an automata learning model (LA) or a GA. They map the decision tree pruning as boolean-vector learning problem. For such, each decision tree is mapped into a 3-layer feedforward neural network. Each node in the first hidden layer consists of one hyperplane (split rule) and its complement. Each node in the second layer represents a leaf node labeled as class 1 . The third layer consists of a single OR unit.

Formally, the first layer has $M$ units, and the $i^{\text {th }}$ unit represents a hyperplane $H_{i}$, parametrized by $\mathbf{w}_{\mathbf{i}}=\left[w_{i 0}, \ldots, w_{i n}\right] \in \Re^{n+1}, 1 \leq i \leq M$, assuming an $n$-dimensional space of attributes. The output of the $i^{t h}$ unit over a given input instance $\mathbf{x}^{\mathbf{l}} \in \Re^{n}$ is $y_{i}=1$ if $\sum_{j=1}^{n} w_{i j} a_{j}\left(x^{l}\right)+w_{i 0}>0$ and $y_{i}=0$ otherwise. 
The second layer has $L$ units, each one implementing an AND function. The $l^{\text {th }}$ unit is connected to all first-layer units through a weight vector $\mathbf{v}_{\mathbf{l}}=\left[v_{l 1}, v_{l 2}, \ldots, v_{l M}\right]$, where $v_{l i} \in$ $\{0,1\} \forall i, 1 \leq i \leq L$.

The output for the $l^{\text {th }}$ unit is $a_{l}=1$ if $\forall i, y_{i}=1$ and $v_{l i}=1$, otherwise $a_{l}=0$. The third layer outputs 1 if at least one of the second-layer unit outputs is 1 , and 0 if all second-layer outputs are 0 (OR operation).

Each second-layer unit is connected (with weight 1) to all hyperplanes in the first layer that appear on the path from the root to the leaf node corresponding to this unit. All the other connections will have weight 0 . It is easy to see that the final output of the network will be 1 on all patterns classified as belonging to class 1 by the decision tree. Given this framework, pruning a decision tree consists of learning the boolean-vector $\mathrm{V}_{1}$ that maximizes some measure (e.g., accuracy).

Note that the hyperplanes in the first-layer are those discovered during the full growth of a decision tree by any oblique decision-tree induction algorithm. Besides, note that the number of second-layer units $(L)$ can be fixed heuristically according to the desired level of pruning. Different values for $\mathrm{V}_{1}$ will lead to different ways to restructure the decision tree. This strategy is much more sophisticated than simply replacing a subtree by a leaf node, because it can lead to a more drastic restructuring of the original tree. A GA is used to evolve a set of fixed-length binary strings that represent the $V_{l}$ vector. The authors mention that they applied "standard" crossover and mutation operations (no further details were informed).

\subsubsection{Other Decision-Tree Components}

Turney (1995) proposes a hybrid approach, ICET (Inexpensive Classification with Expensive Tests), where a GA is used to evolve a population of biases for a decision-tree induction algorithm (variation of Quinlan's C4.5). ICET considers both the costs of tests (i.e., cost of attributes) and the costs of classification errors.

In ICET, instead of using C4.5's gain ratio, attributes are evaluated by the information cost function (ICF) (Nunez, 1991), defined for each attribute $i$ as follows:

$$
I C F_{i}=\frac{2^{\Delta I_{i}}-1}{\left(C_{i}+1\right)^{\alpha}}
$$

where $\Delta I_{i}$ is the information gain associated to the $i^{t h}$ attribute when splitting a given portion of the data set, $C_{i}$ is the cost of measuring the $i^{\text {th }}$ attribute and $\alpha=[0,1]$ is the strength of the bias towards lower cost attributes. Note that $\alpha=0$ means the attribute cost is ignored and maximizing $I C F$ is equivalent to maximizing $\Delta I_{i}$, whereas $\alpha=1$ means $I C F$ is strongly biased by cost.

ICET evolves a population of individuals encoded as a fixed-length binary string consisting of $12 \times n+16$ bits, where $n$ is the number of data set attributes. Each attribute cost $C_{i}$ in (4.23) is encoded as a 12-bit value. In addition, each string also encodes the parameter $\alpha$ in (4.23) and 
the pruning confidence factor for $\mathrm{C} 4.5$, both as 8-bit values. ICET uses a random initialization of individuals, a crossover rate of 0.6 and a mutation rate of 0.001 .

The fitness function is the average cost classification - the sum of attribute and classification costs, for all validation instances, divided by the total number of validation instances. Attribute costs in a same subtree are only counted once.

Bratu et al. (2007) propose extending ICET by modifying components of the original GA. By introducing elitism, increasing the search variability factor, and extending the number of iterations, the proposed extension manages to outperform other cost-sensitive algorithms, even for data sets on which the initial implementation yielded modest results. Results appear to suggest there is an urge for a more comprehensive analysis on the impact of varying the GA parameters, as it is empirically demonstrated that such changes can lead to significant performance improvements.

\subsection{Applications of EAs for Decision-Tree Induction}

Considering that most studeis focus on the EAs ability for dealing with the drawbacks of the greedy strategy, usually these approaches are validated on public benchmark data sets for comparison purposes, instead of focusing on a specific application domain. Nevertheless, there are some papers that limit their scope to a specific domain. In these cases, we can highlight the application areas listed in Table 4.1, whose EAs are briefly reviewed next.

Table 4.1: Application Areas of Evolutionary Decision Trees.

\begin{tabular}{l|l}
\hline Application area & References \\
\hline Astronomy & (Cantu-Paz et al., 2000) \\
Cold Mill Strip & (Kim et al., 2004a,b) \\
Character Recognition & (Haruyama and Zhao, 2002; Oka and Zhao, 2000; Shirasaka et al., 1998; Tanigawa and Zhao, 2000; Zhao \\
and Shirasaka, 1999) \\
Finance & (Kuo et al., 2007) \\
Marketing & (Fu et al., 2003b; Sorensen and Janssens, 2003) \\
Medicine & (Cuong and Pham, 2009; Estrada-Gil et al., 2007; Kuo et al., 2008; Podgorelec et al., 2005; Podgorelec and \\
Natural Language Processing & Kokol, 1998, 2001b; Smith, 2008; Zorman et al., 2001) \\
Software Engineering & (Siegel, 1994) \\
\hline
\end{tabular}

In (Cantu-Paz et al., 2000), a GA for decision-tree induction is applied to an astronomy problem, the classification of galaxies with a bent-double morphology. This EA is the same proposed in (Cantu-Paz and Kamath, 2000) for inducing oblique decision trees. Kim et al. (2004a,b) present a method for recognizing various defect patterns of a cold mill strip by using evolutionary binary decision trees.

Several EAs evolve binary decision trees for character recognition (classification). Work like (Haruyama and Zhao, 2002; Oka and Zhao, 2000; Shirasaka et al., 1998; Tanigawa and Zhao, 2000; Zhao and Shirasaka, 1999) evolve binary decision trees using GAs and later apply the evolved decision tree to digit recognition tasks. Kuo et al. (2007) evolve decision trees through GP and analyse their performance over a credit card data set. 
Several EAs evolve decision trees for specific medical problems. For instance, Podgorelec and Kokol (1998) investigate a multi-objective weighted-formula based on GAs for decision-tree induction for detecting cardiac prolapse. Its fitness function incorporates domain knowledge by not considering all errors equally. The most costly errors are made when a patient with cardiac prolapse or silent prolapse is classified as with no prolapse. Conversely, it is not so costly when healthy patients are classified as with prolapse or silent prolapse.

In (Podgorelec and Kokol, 2001b), the authors propose an integrated computerized environment, DIAPRO, which is a computer tool based on EAs for medical diagnosis. DIAPRO attempts to improve accuracy, sensitivity and specificity for decision making using evolutionary generated decision trees. In (Zorman et al., 2001), a real-world orthopedic fracture data set is investigated through multiple approaches of decision-tree induction, and it is shown that the evolutionary approach presents the best compromise among the measures evaluated.

In (Podgorelec et al., 2005), the authors present a new outlier prediction system for improving the classification performance in medical data mining. Two cardiovascular data sets are investigated. The method introduces the class confusion score metric that is based on the classification results of a set of classifiers. These classifiers are generated by the EA proposed in (Podgorelec and Kokol, 2002), and the main idea is to verify if there is any inconsistence when classifying a specific example through different classifiers. In (Cuong and Pham, 2009) a GP evolves decision trees for cardiac diagnoses. The GP was tested by using cardiac single proton emission computed tomography images.

In (Estrada-Gil et al., 2007), a GP evolves decision trees to detect interactions in genetic variants. Preliminary experiments using GPDTI have been able to find a 3-marker interaction in a data set of 1000 markers and a sample size of 600 subjects. Smith (2008) evolved decision trees in a bioinformatics application, where they used a GA for finding RNA family-specific decision trees. This work is well adapted to the application domain, i.e.both individual representation and fitness function are domain-specific.

Siegel (1994) proposes evolving decision trees for the problem of word sense disambiguation. Results indicate that evolved decision trees often include rules that provide insightful hints for linguists.

Many studies propose evolving decision trees for software engineering tasks. For instance, in (Kokol et al., 2001) evolutionary decision trees are used as a software fault predictive approach. It is shown that software complexity measures can be successfully used to induce evolutionary decision trees for predicting dangerous modules (with many undetected faults). The authors recommend redesigning the fault-detected modules or devoting more testing or maintenance effort to them in order to enhance the software quality and reliability. In (Podgorelec and Kokol, 2002), Podgorelec and Kokol present a self-adapting EA for decision-tree induction and its application for predicting faults in dangerous software modules. Khoshgoftaar et al. (2003) present an automated and simplified GP-based decision tree modeling technique for the software quality classification problem. The proposed technique is based on multi-objective optimisation using strongly typed 
GP. In the context of and industrial high-assurance software system, two fitness functions are used: one for minimizing the predictive error and another for parsimony.

Khoshgoftaar and Liu (2007) propose a GP-based multi-objective optimisation modeling technique for calibrating a goal-oriented software quality classification model geared toward a cost-effective resource utilization. It is shown that the proposed model achieves good performance in the context of optimisation of the three modeling objectives: 1) minimizing a modified expected cost of misclassification measure for software quality classification models; 2) enabling the number of predicted fault-prone modules to be equal to the number of modules which can be inspected by the allocated resources; and 3) controlling the size of the decision tree to facilitate comprehensibility in model interpretation, and providing faster GP-runs. In (Chis, 2008), the authors present an evolutionary approach for decision-tree induction and its application for classifying software modules as defective or defect-free. A set of 21 predictive attributes, containing various software complexity measures and metrics for each software module was used. The authors concluded that the discovered decision rules are useful for developing new rules in software defect identification.

\subsection{Chapter Remarks}

Decision trees are one of the most frequently used representations for classifiers. A very large number of articles has been devoted to this classification method. Whereas most well-known algorithms for decision-tree induction rely on a greedy divide-and-conquer strategy for partitioning the tree, alternative approaches have become more and more common in the past few years. More specifically, a fairly large amount of studies have been dedicated to the evolutionary induction of decision trees.

We presented in this chapter a survey of papers which combine evolutionary algorithms and decision trees. We proposed a taxonomy to better organise the work in this area, conveniently separating research that evolve decision trees from research that evolve components of decision-tree algorithms. Moreover, we documented every important step of an evolutionary algorithm for decision-tree induction and component evolution, so the interested reader can easily design its own EA for decision trees according to an extensive enumeration of design options and strategies. We presented, when suitable, criticism over specific choices and also guidelines for handling problems that will eventually come up when designing evolutionary decision trees. Next, we make a brief critical analysis on the advantages and disadvantages of evolutionary induction of decision trees, and we finalize this survey by pointing out tendencies of future work in the area.

\subsubsection{The benefits of evolutionary decision trees}

The main benefit of evolving decision trees is EAs's ability to escape from local optima. EAs are able to perform a robust global search in the space of candidate solutions, and thus they are 
less susceptible to local optima convergence. In addition, as a result of this global search, EAs tend to cope better with attribute interactions than greedy methods (Freitas, 2002), which means that complex attribute relationships which are not detected during the greedy evaluation can be discovered by an EA when evolving decision trees.

Another clear advantage of evolving decision trees is the possibility of explicitly biasing the search space through multi-objective optimisation. EAs can naturally optimise multi-objectives, which may be crucial in several application domains. For instance, cost sensitive classification - which is the case of most medical classification problems - can be greatly enhanced by explicit optimizing measures that address different error costs. Parsimony pressure is yet another important feature that is easily implemented in a multi-objective EA for decision-tree induction.

\subsubsection{The drawbacks of evolutionary decision trees}

The main disadvantage of evolutionary induction of decision trees is related to time constraints. EAs are a solid but computationally expensive heuristic. Nevertheless, faster and faster computational resources have allowed EAs to be increasingly used in a variety of applications over the years. Advances on parallel processing have also enabled EAs to be better explored in acceptable execution times. It should also be noted that, in real-world problems, the time spent preparing the data for data mining purposes tends to be much larger than the time spent by inducing a classification or regression model. Hence, in real-world problems, the long processing time of evolutionary algorithms tends not to be the bottleneck of the entire knowledge discovery process.

Another disadvantage is the large number of parameters that must be tuned in order to run a full execution of an EA. Espejo et al. (2010) acknowledge this problem in GP-based classifiers, though they reckon it is a secondary one. The same difficulty occurs for genetic algorithms, since GAs and GP share much of the same parameters.

\subsubsection{What is next on evolutionary induction of decision trees?}

The theoretical assumption that evolved decision trees can provide better prediction results than greedy-designed ones has been confirmed empirically in most of the papers reviewed in this survey. Nevertheless, this advantage comes with a price. Most strategies reviewed here point out that evolving decision trees is a costly, time-consuming task. Not much is said on how this can be alleviated. Parallel implementations are usually the suggestion for speeding up decision tree evolution, even though very few studies actually implement parallel algorithms or care to make a comparison on the time savings. We believe a future trend on evolutionary induction of decision trees is concerned on the analysis of techniques to speed up evolution. A recent work by Kalles and Papagelis (2010) proposes time savings in evolutionary decision-tree induction through fitness inheritance. They reckon that storing instance indices at leaf nodes is enough for fitness to be piecewise computed in a lossless fashion, thus leading to substantial speed-ups within the 
evolutionary cycle. They show the derivation of the expected speed-up on two bounding cases and their claims are supported by an extensive empirical analysis. We believe that further exploration of this kind of analysis is beneficial in order to establish evolutionary decision-tree induction as a strong alternative to greedy methods.

Synthetic data evaluation is a methodology that could be more investigated by researchers of the field. It allows for a more theoretical exploration that is complementary to the prevailing empirical evaluation of EAs for decision-tree induction. Examples of papers that make use of such a methodology are (Kennedy et al., 1997; Kim, 2004; Kretowski and Grzes, 2006b; Llorà and Wilson, 2004; Papagelis and Kalles, 2001; Slavov and Nokolaev, 1997). In (Slavov and Nokolaev, 1997), for example, instances were artificially generated in order to produce data sets with specific characteristics, such as representative enough categorical distributions and extreme cases of easiness or hardness for decision-tree induction.

Another future trend is the development of data-based parameter guidelines. Researchers on the area should start establishing the relationship between EAs' parameter settings and data sets' features. For that, we believe that the study of data set complexity measures such as those proposed by Ho and Basu (2002); Ho et al. (2006) may be beneficial for understanding the geometrical shape of classification data sets. Once the complexity of classification data is fully-understood, we believe it would be possible for researchers to recommend parameter values for decision-tree evolution backed up by theoretical and empirical evidence.

Finally, we believe that an important and challenging future trend in this area concerns evolving decision-tree induction algorithms, which is precisely the main goal of this thesis. In such an approach, the EA evolves a full decision-tree algorithm (with loops and procedures for growing, pruning and evaluating candidate decision trees), rather than just evolving a decision-tree classifier. One of the advantages of this strategy is the possibility of tailoring an algorithm for a given domain, and instead of using a generic decision-tree induction algorithm (such as C4.5), we can make use of an algorithm that was explicitly designed to be efficient in specific data sets. This approach is first introduced in the next chapter. 



\section{HEAD-DT: Automatic Design of Decision-Tree Algorithms}

As presented in Chapter 2, for the past 40 years researchers have attempted to improve decision-tree induction algorithms, either by proposing new splitting criteria for internal nodes, by investigating pruning strategies for avoiding overfitting, by testing new approaches for dealing with missing values, or even by searching for alternatives to the top-down greedy induction. Each new decision-tree induction algorithm presents some (or many) of these strategies, which are chosen in order to maximize performance in empirical analyses. Nevertheless, the number of different strategies for the several components of a decision-tree algorithm is so vast after these 40 years of research that it would be impracticable for a human being to test all possibilities with the purpose of achieving the best performance in a given data set (or in a set of data sets). Hence, we pose two questions for researchers in the area: "is it possible to automate the design of decision-tree induction algorithms?", and, if so, "how can we automate the design of a decision-tree induction algorithm?"

The answer for these questions arose with the pioneering work of Pappa and Freitas (2006); Pappa (2007), which proposed the automatic design of rule induction algorithms through an evolutionary algorithm. The authors proposed the use of a grammar-based GP algorithm for building and evolving individuals which are, in fact, rule induction algorithms. This approach successfully employs EAs to evolve a generic rule induction algorithm, which can then be applied to solve many different classification problems, instead of evolving a specific set of rules tailored to a particular data set. 
As presented in Chapter 3, in the area of optimisation this type of approach is named hyper-heuristics (HHs) (Burke and Petrovic, 2002; Burke et al., 2003b). HHs are search methods for automatically selecting and combining simpler heuristics, resulting in a generic heuristic that is used to solve any instance of a given optimisation problem. For instance, a $\mathrm{HH}$ can generate a generic heuristic for solving any instance of the timetabling problem (i.e., allocation of any number of resources subject to any set of constraints in any schedule configuration) whilst a conventional EA would just evolve a solution to one particular instance of the timetabling problem (i.e., a predefined set of resources and constraints in a given schedule configuration).

In this chapter, we present our hyper-heuristic strategy for automatically designing decision-tree induction algorithms, namely HEAD-DT (Hyper-Heuristic Evolutionary Algorithm for Automatically Designing Decision-Tree Algorithms). Section 5.1 introduces HEAD-DT and its evolutionary scheme. Section 5.2 presents the individual representation adopted by HEAD-DT to evolve decision-tree algorithms, as well as information regarding each individual's gene. Section 5.3 shows the evolutionary cycle of HEAD-DT, detailing its genetic operators. Section 5.4 depicts the fitness evaluation process in HEAD-DT, and introduces two possible frameworks for executing HEAD-DT. Section 5.5 computes the total size of the search space that HEAD-DT is capable of traversing, whereas Section 5.6 discusses related work.

\subsection{Introduction}

The main objective of this thesis is to automatically design decision-tree induction algorithms. For such, we designed a hyper-heuristic evolutionary algorithm named HEAD-DT (Hyper-Heuristic Evolutionary Algorithm for Automatically Designing Decision-Tree Algorithms).

According to the definition by Burke et al. (2010), "a hyper-heuristic is an automated methodology for selecting or generating heuristics to solve hard computational search problems". Hyper-heuristics can automatically generate new heuristics suited to a given problem or class of problems. This is carried out by combining components or building-blocks of human-designed heuristics. The motivation behind hyper-heuristics is to raise the level of generality at which search methodologies can operate. In the context of decision trees, instead of searching through an EA for the best decision tree to a given problem (regular metaheuristic approach, e.g., (Barros et al., 2011a,c)), the generality level is raised by searching for the best decision-tree induction algorithm that may be applied to several different problems (hyper-heuristic approach).

HEAD-DT can be seen as a regular generational EA in which individuals are collections of building blocks of top-down decision-tree induction algorithms. We employ typical operators from EAs, like tournament selection, mutually-exclusive genetic operators (reproduction, crossover, and mutation) and a typical stopping criterion that halts evolution after a predefined number of generations. The evolution of individuals in HEAD-DT follows the scheme presented in Figure 5.1. 


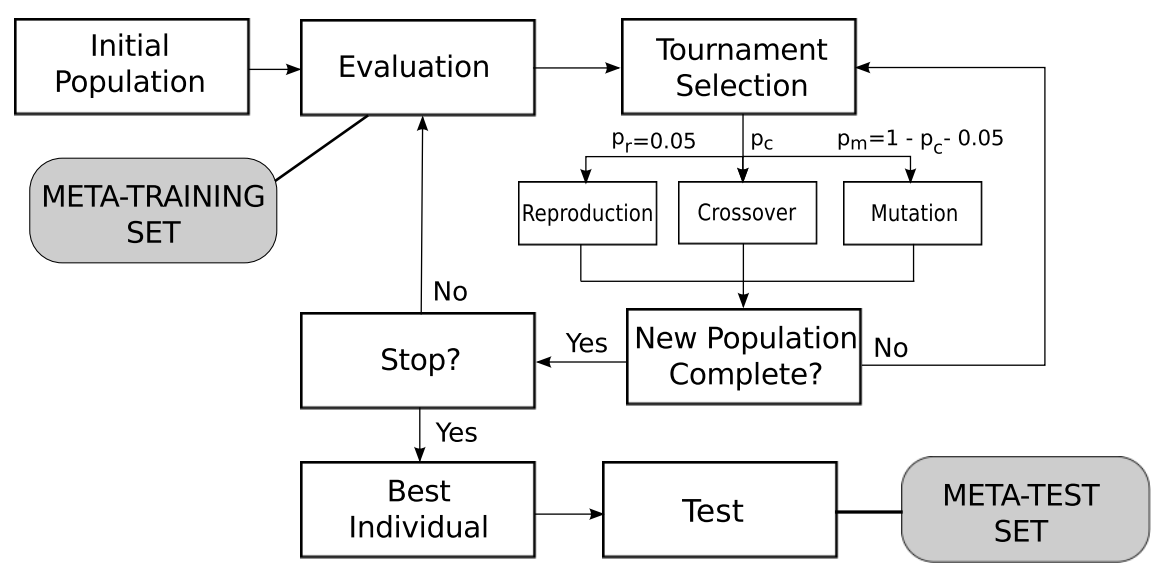

Figure 5.1: HEAD-DT's evolutionary scheme.

\subsection{Individual Representation}

Each individual in HEAD-DT is encoded as an integer vector, as depicted in Figure 5.2, and each gene has a different range of supported values. We divided the genes into four categories, representing the major building blocks (design components) of a decision-tree induction algorithm:

- split genes;

- stopping criteria genes;

- missing values genes;

- pruning genes.

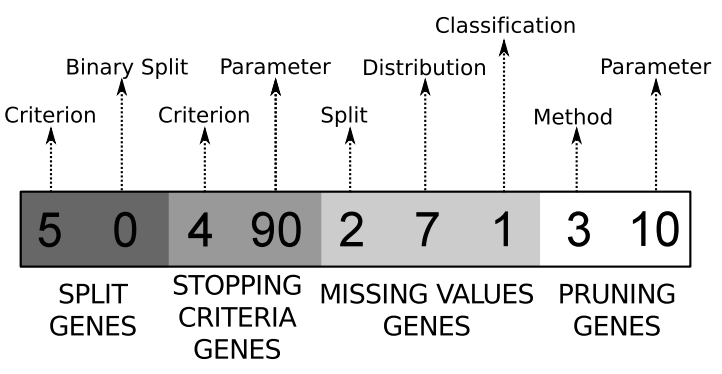

Figure 5.2: Linear-genome for evolving decision-tree algorithms.

\subsubsection{Split Genes}

The linear genome that encodes individuals in HEAD-DT holds two genes for the split component of decision trees. These genes represent the design component that is responsible for selecting the attribute to split the data in the current node of the decision tree. Based on the 
Table 5.1: Split criteria implemented in HEAD-DT.

\begin{tabular}{ll}
\hline Criterion & Reference \\
\hline Information Gain & (Quinlan, 1986) \\
Gini Index & (Breiman et al., 1984) \\
Global Mutual Information & (Gleser and Collen, 1972) \\
G Statistic & (Mingers, 1987) \\
Mantáras Criterion & (De Mántaras, 1991) \\
Hypergeometric Distribution & (Martin, 1997) \\
Chandra-Varghese Criterion & (Chandra and Varghese, 2009) \\
DCSM & (Chandra et al., 2010) \\
$\chi^{2}$ & (Mingers, 1989b) \\
Mean Posterior Improvement & (Taylor and Silverman, 1993) \\
Normalized Gain & (Jun et al., 1997) \\
Orthogonal Criterion & (Fayyad and Irani, 1992) \\
Twoing & (Breiman et al., 1984) \\
CAIR & (Ching et al., 1995) \\
Gain Ratio & (Quinlan, 1993) \\
\hline
\end{tabular}

selected attribute, a decision rule is generated for filtering the input data in subsets, and the process continues recursively.

To model this design component, we make use of two different genes. The first one, criterion, is an integer that indexes one of the 15 splitting criteria that are implemented in HEAD-DT (see Table 5.1). The most successful criteria are based on Shannon's entropy (Shannon, 1948), a concept well-known in information theory. Entropy is a unique function that satisfies the four axioms of uncertainty. It represents the average amount of information when coding each class into a codeword with ideal length according to its probability. Examples of splitting criteria based on entropy are global mutual information (GMI) (Gleser and Collen, 1972) and information gain (Quinlan, 1986). The latter is employed by Quinlan in his ID3 system (Quinlan, 1986). However, Quinlan points out that information gain is biased towards attributes with many values, and thus proposes a solution named gain ratio (Quinlan, 1993). Gain ratio normalizes the information gain by the entropy of the attribute being tested. Several variations of the gain ratio have been proposed, such as the normalized gain (Jun et al., 1997).

Alternatives to entropy-based criteria are the class of distance-based measures. These criteria evaluate separability, divergency, or discrimination between classes. Examples are the Gini index (Breiman et al., 1984), the twoing criterion (Breiman et al., 1984), the orthogonality criterion (Fayyad and Irani, 1992), among several others. We also implemented as options for HEAD-DT lesser-known criteria such as CAIR (Ching et al., 1995) and mean posterior improvement (Taylor and Silverman, 1993), as well as the more recent Chandra-Varghese (Chandra and Varghese, 2009) and DCSM (Chandra et al., 2010), to enhance the diversity of options for generating splits in a decision tree.

The second gene that controls the split component of a decision-tree algorithm is binary split. It is a binary gene that indicates whether the splits of a decision tree are going to be binary or multi-way. In a binary tree, every split has only two outcomes, which means that nominal attributes with many categories are aggregated into two subsets. In a multi-way tree, nominal attributes are divided according to their number of categories - one edge (outcome) for each category. In both 
cases, numeric attributes always partition the tree in two subsets, represented by tests att $\leq \Delta$ and att $>\Delta$.

\subsubsection{Stopping Criteria Genes}

The top-down induction of decision trees is recursive and it continues until a stopping criterion (also known as pre-pruning) is satisfied. The linear genome in HEAD-DT holds two genes for representing this design component: criterion and parameter.

The first gene, criterion, selects among the following five different strategies for stopping the tree growth:

1. Reaching class homogeneity: when every instance that reaches a given node belong to the same class, there is no reason to split this node any further. This strategy can be the only single stopping criterion, or it can be combined with the next four strategies;

2. Reaching the maximum tree depth: a parameter tree depth can be specified to avoid deep trees. Range: $[2,10]$ levels;

3. Reaching the minimum number of instances for a non-terminal node: a parameter minimum number of instances for a non-terminal node can be specified to avoid/alleviate the data fragmentation problem in decision trees. Range: $[1,20]$ instances;

4. Reaching the minimum percentage of instances for a non-terminal node: same as above, but instead of the actual number of instances, a percentage of instances is defined. The parameter is thus relative to the total number of instances in the training set. Range: $[1 \%, 10 \%]$ the total number of instances;

5. Reaching an accuracy threshold within a node: a parameter accuracy reached can be specified for halting the growth of the tree when the accuracy within a node (majority of instances) has reached a given threshold. Range: $\{70 \%, 75 \%, 80 \%, 85 \%, 90 \%, 95 \%, 99 \%\}$ accuracy.

Gene parameter dynamically adjusts a value in the range $[0,100]$ to the corresponding strategy. For example, if the strategy selected by gene criterion is reaching the maximum tree depth, the following mapping function is executed:

$$
\text { param }=(\text { value } \bmod 9)+2
$$

This function maps from $[0,100]$ (variable value) to [2,10] (variable param), which is the desired range of values for the parameter of strategy reaching the maximum tree depth. Similar mapping functions are executed dynamically to adjust the ranges of gene parameter. 


\subsubsection{Missing Values Genes}

The next design component of decision trees that is represented in the linear genome of HEAD-DT is the missing value treatment. Missing values may be an issue during tree induction and also during classification. We make use of three genes to represent missing values strategies in different moments of the induction/deduction process. During tree induction, there are two moments in which we need to deal with missing values: splitting criterion evaluation (split gene), and instances distribution (distribution gene). During tree deduction (classification), we may also have to deal with missing values in the test set (classification gene).

During the split criterion evaluation in node $t$ based on attribute $a_{i}$, we implemented the following strategies:

- Ignore all instances whose value of $a_{i}$ is missing (Breiman et al., 1984; Friedman, 1977);

- Imputation of missing values with either the mode (nominal attributes) or the mean/median (numeric attributes) of all instances in $t$ (Clark and Niblett, 1989);

- Weight the splitting criterion value (calculated in node $t$ with regard to $a_{i}$ ) by the proportion of missing values (Quinlan, 1989);

- Imputation of missing values with either the mode (nominal attributes) or the mean/median (numeric attributes) of all instances in $t$ whose class attribute is the same of the instance whose $a_{i}$ value is being imputed.

For deciding which child node training instance $x_{j}$ should go to, considering a split in node $t$ over $a_{i}$, we adopted the options:

- Ignore instance $x_{j}$ (Quinlan, 1986);

- Treat instance $x_{j}$ as if it has the most common value of $a_{i}$ (mode or mean), regardless of the class (Quinlan, 1989);

- Treat instance $x_{j}$ as if it has the most common value of $a_{i}$ (mode or mean) considering the instances that belong to the same class than $x_{j}$;

- Assign instance $x_{j}$ to all partitions (Friedman, 1977);

- Assign instance $x_{j}$ to the partition with largest number of instances (Quinlan, 1989);

- Weight instance $x_{j}$ according to the partition probability (Kononenko et al., 1984; Quinlan, 1993);

- Assign instance $x_{j}$ to the most probable partition, considering the class of $x_{j}$ (Loh and Shih, 1997). 
Finally, for classifying an unseen test instance $x_{j}$, considering a split in node $t$ over $a_{i}$, we used the strategies:

- Explore all branches of $t$ combining the results (Quinlan, 1987a);

- Take the route to the most probable partition (largest subset);

- Halt the classification process and assign instance $x_{j}$ to the majority class of node $t$ (Quinlan, 1989).

\subsubsection{Pruning Genes}

Pruning was originally conceived as a strategy for tolerating noisy data, though it was found to improve decision tree accuracy in many noisy data sets (Breiman et al., 1984; Quinlan, 1986, 1987b). It has now become an important part of greedy top-down decision-tree induction algorithms. HEAD-DT holds two genes for this design component. The first gene, method, indexes one of the five well-known approaches for pruning a decision tree presented in Table 5.2, and also the option of not pruning at all. The second gene, parameter, is in the range $[0,100]$ and its value is again dynamically mapped by a function according to the selected pruning method.

Table 5.2: Pruning methods implemented in HEAD-DT.

\begin{tabular}{ll}
\hline Method & Reference \\
\hline Reduced-error pruning & (Quinlan, 1987b) \\
Pessimistic error pruning & (Quinlan, 1987b) \\
Minimum error pruning & (Cestnik and Bratko, 1991; Niblett and Bratko, 1986) \\
Cost-complexity pruning & (Breiman et al., 1984) \\
Error-based pruning & (Quinlan, 1993) \\
\hline
\end{tabular}

1) Reduced-error pruning (REP) is a conceptually simple strategy proposed by Quinlan (1987b). It uses a pruning set (a part of the training set) to evaluate the goodness of a given subtree from $T$. The idea is to evaluate each non-terminal node $t$ with regard to the classification error in the pruning set. If such an error decreases when we replace the subtree $T^{(t)}$ rooted on $t$ by a leaf node, then $T^{(t)}$ must be pruned. Quinlan imposes a constraint: a node $t$ cannot be pruned if it contains a subtree that yields a lower classification error in the pruning set. The practical consequence of this constraint is that REP should be performed in a bottom-up fashion. The REP pruned tree $T^{\prime}$ presents an interesting optimality property: it is the smallest most accurate tree resulting from pruning original tree $T$ (Quinlan, 1987b). Besides this optimality property, another advantage of REP is its linear complexity, since each node is visited only once in $T$. A clear disadvantage is the need of using a pruning set, which means one has to divide the original training set, resulting in less instances to grow the tree. This disadvantage is particularly serious for small data sets. For REP, the parameter gene is regarding the percentage of training data to be used in the pruning set (varying within the interval $[10 \%, 50 \%]$ ). 
2) Also proposed by Quinlan (1987b), the pessimistic error pruning (PEP) uses the training set for both growing and pruning the tree. The apparent error rate, i.e., the error rate calculated over the training set, is optimistically biased and cannot be used to decide whether pruning should be performed or not. Quinlan thus proposes adjusting the apparent error according to the continuity correction for the binomial distribution in order to provide a more realistic error rate. PEP is computed in a top-down fashion, and if a given node $t$ is pruned, its descendants are not examined, which makes this pruning strategy quite efficient in terms of computational effort. Esposito et al. (1997) point out that the introduction of the continuity correction in the estimation of the error rate has no theoretical justification, since it was never applied to correct over-optimistic estimates of error rates in statistics. For PEP, the parameter gene is the number of standard errors (SEs) to adjust the apparent error, in the set $\{0.5,1,1.5,2\}$.

3) Originally proposed by Niblett and Bratko (1986) and further extended in (Cestnik and Bratko, 1991), minimum error pruning (MEP) is a bottom-up approach that seeks to minimize the expected error rate for unseen cases. It uses an ad-hoc parameter $m$ for controlling the level of pruning. Usually, the higher the value of $m$, the more severe the pruning. Cestnik and Bratko (1991) suggest that a domain expert should set $m$ according to the level of noise in the data. Alternatively, a set of trees pruned with different values of $m$ could be offered to the domain expert, so he/she can choose the best one according to his/her experience. For MEP, the parameter gene comprises variable $m$, which may range within $[0,100]$.

4) Cost-complexity pruning (CCP) is the post-pruning strategy adopted by the CART system (Breiman et al., 1984). It consists of two steps: (i) generate a sequence of increasingly smaller trees, beginning with $T$ and ending with the root node of $T$, by successively pruning the subtree yielding the lowest cost complexity, in a bottom-up fashion; (ii) choose the best tree among the sequence based on its relative size and accuracy (either on a pruning set, or provided by a cross-validation procedure in the training set). The idea within step 1 is that pruned tree $T_{i+1}$ is obtained by pruning the subtrees that show the lowest increase in the apparent error (error in the training set) per pruned leaf. Regarding step 2, CCP chooses the smallest tree whose error (either on the pruning set or on cross-validation) is not more than one standard error (SE) greater than the lowest error observed in the sequence of trees. For CCP, there are two parameters that need to be set: the number of SEs (in the same range than PEP) and the pruning set size (in the same range than REP).

5) Error-based pruning (EBP) was proposed by Quinlan and it is implemented as the default pruning strategy of C4.5 (Quinlan, 1993). It is an improvement over PEP, based on a far more pessimistic estimate of the expected error. Unlike PEP, EBP performs a bottom-up search, and it carries out not only the replacement of non-terminal nodes by leaves but also grafting of subtree $T^{(t)}$ onto the place of parent $t$. For deciding whether to replace a non-terminal node by a leaf (subtree replacement), to graft a subtree onto the place of its parent (subtree raising) or not to prune at all, a pessimistic estimate of the expected error is calculated by using an upper confidence bound. An advantage of EBP is the new grafting operation that allows pruning useless branches 
without ignoring interesting lower branches. A drawback of the method is the presence of an ad-hoc parameter, $C F$. Smaller values of $C F$ result in more pruning. For EBP, the parameter gene comprises variable $C F$, which may vary within $[1 \%, 50 \%]$.

\subsubsection{Example of Algorithm Evolved by HEAD-DT}

The linear genome of an individual in HEAD-DT is formed by the building blocks described in the earlier sections: [split criterion, split type, stopping criterion, stopping parameter, pruning strategy, pruning parameter, $m v$ split, $m v$ distribution, $m v$ classification]. One possible individual encoded by that linear string is $[4,1,2,77,3,91,2,5,1]$, which accounts for Algorithm 4.

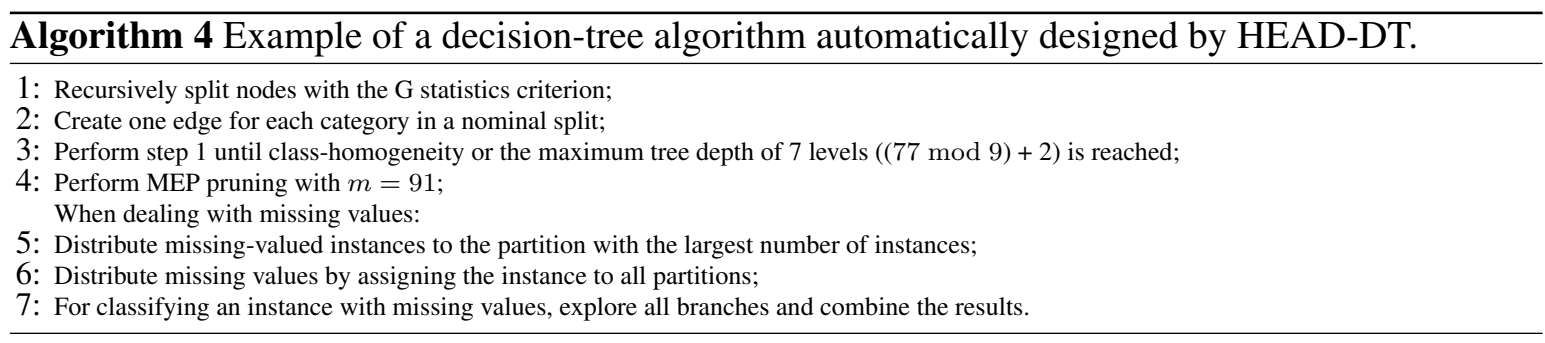

\subsection{Evolution}

The first step of HEAD-DT is the generation of the initial population, in which a population of 100 individuals (default value) is randomly generated (generation of random numbers within the genes' acceptable range of values). Next, the population's fitness is evaluated based on the data sets that belong to the meta-training set. The individuals then participate of a pairwise tournament selection procedure ( $t=2$ is the default parameter) for defining those that will undergo the genetic operators. Individuals may participate in either uniform crossover, random uniform gene mutation, or reproduction, the three mutually-exclusive genetic operators employed in HEAD-DT (see Figure 5.3).

The uniform crossover is guided by a swap probability $p_{s}$ (default value $=0.5$ ) that ultimately indicates whether a child's gene should come from parent $A$ or from parent $B$. Algorithm 5 depicts the pseudocode of the uniform crossover operator implemented in HEAD-DT.

The mutation operator implemented in HEAD-DT is the random uniform gene mutation. It is guided by a replacement probability $p_{\text {rep }}$ (default value $=0.5$ ), which dictates whether or not a gene's value should be replaced by a randomly generated value within the accepted range of the respective gene. Algorithm 6 depicts the pseudocode of the random uniform gene mutation operator implemented in HEAD-DT.

Finally, reproduction is the operation that copies (clones) a given individual to be part of the EA's next generation in a straightforward fashion. A single parameter $p$ controls the mutually-exclusive genetic operators: crossover probability is given by $p$, whereas mutation 


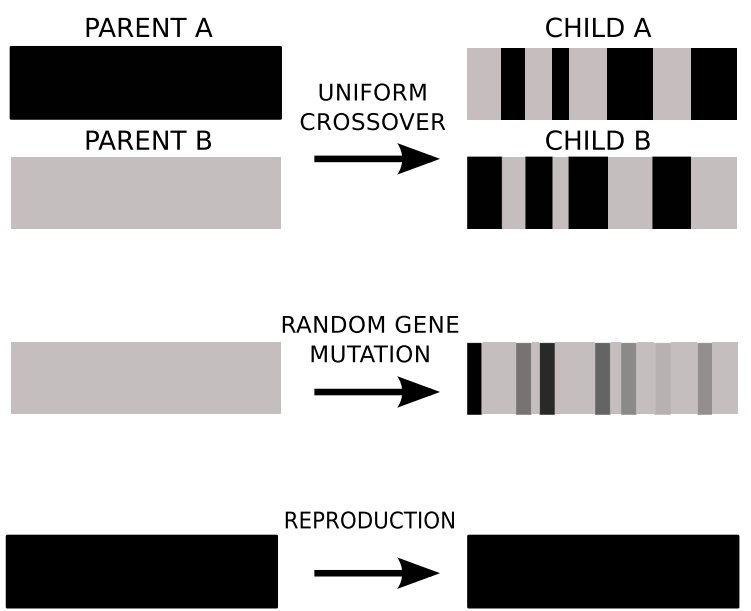

Figure 5.3: HEAD-DT's genetic operators.
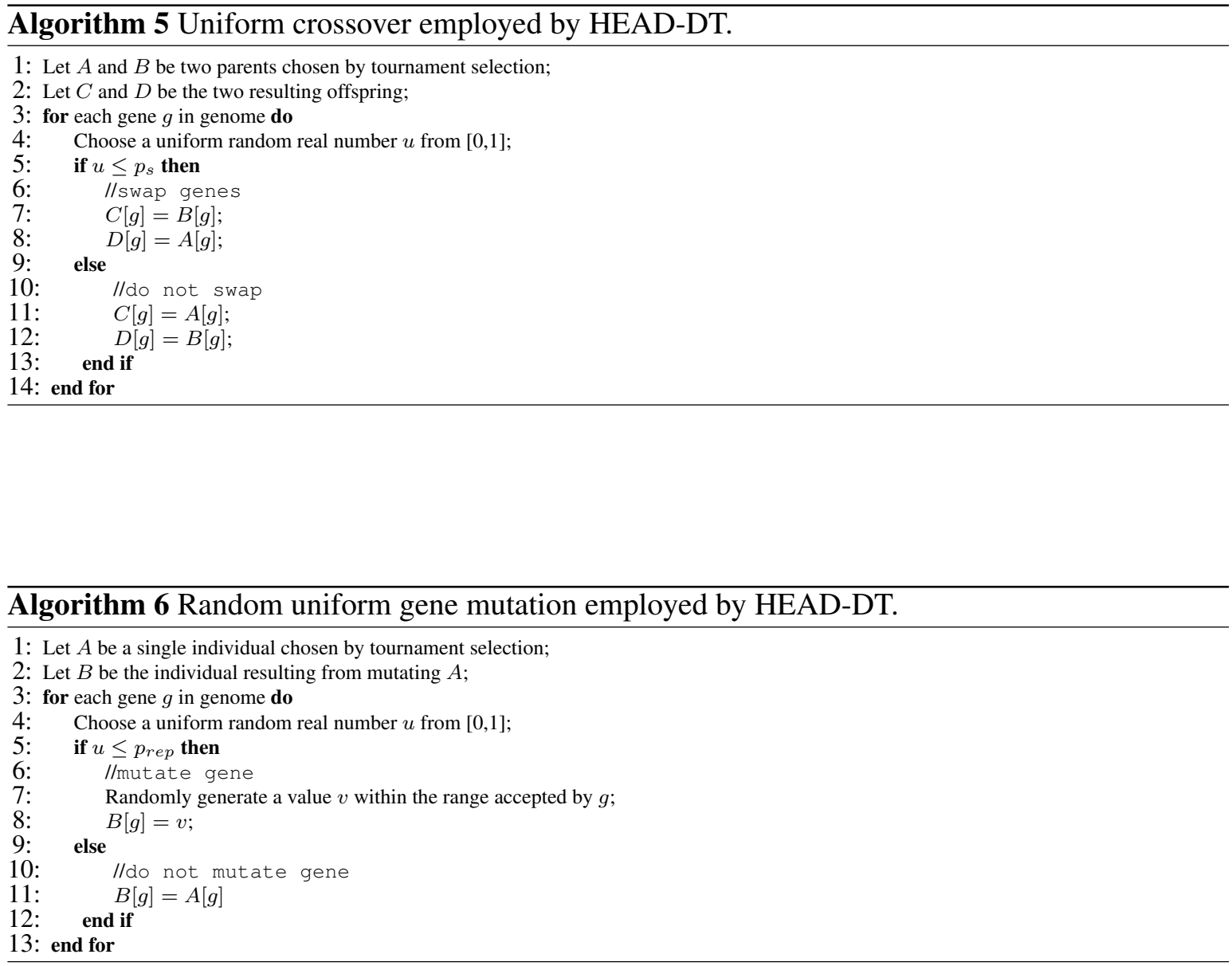
probability is given by $(1-p)-0.05$, and reproduction probability is fixed in 0.05 . For instance, if $p=0.9$, then HEAD-DT is executed with a crossover probability of $90 \%$, mutation probability of $5 \%$ and reproduction probability of 5\%. HEAD-DT employs an elitist strategy, in which the best $e \%$ individuals are kept from one generation to the next $(e=5 \%$ of the population is the default parameter). Evolution ends after a predefined number of generations is achieved ( 100 generations is the default value), and the best individual returned by HEAD-DT is then executed over the meta-test set, so its performance in unseen data can be properly assessed.

Figure 5.4 presents an example of how linear genomes are decoded into algorithms, and how they participate of the evolutionary cycle. For decoding the individuals, the building blocks (indexed components and their respective parameters) are identified, and this information is passed to a skeleton decision-tree induction class, filling the gaps with the selected building blocks.

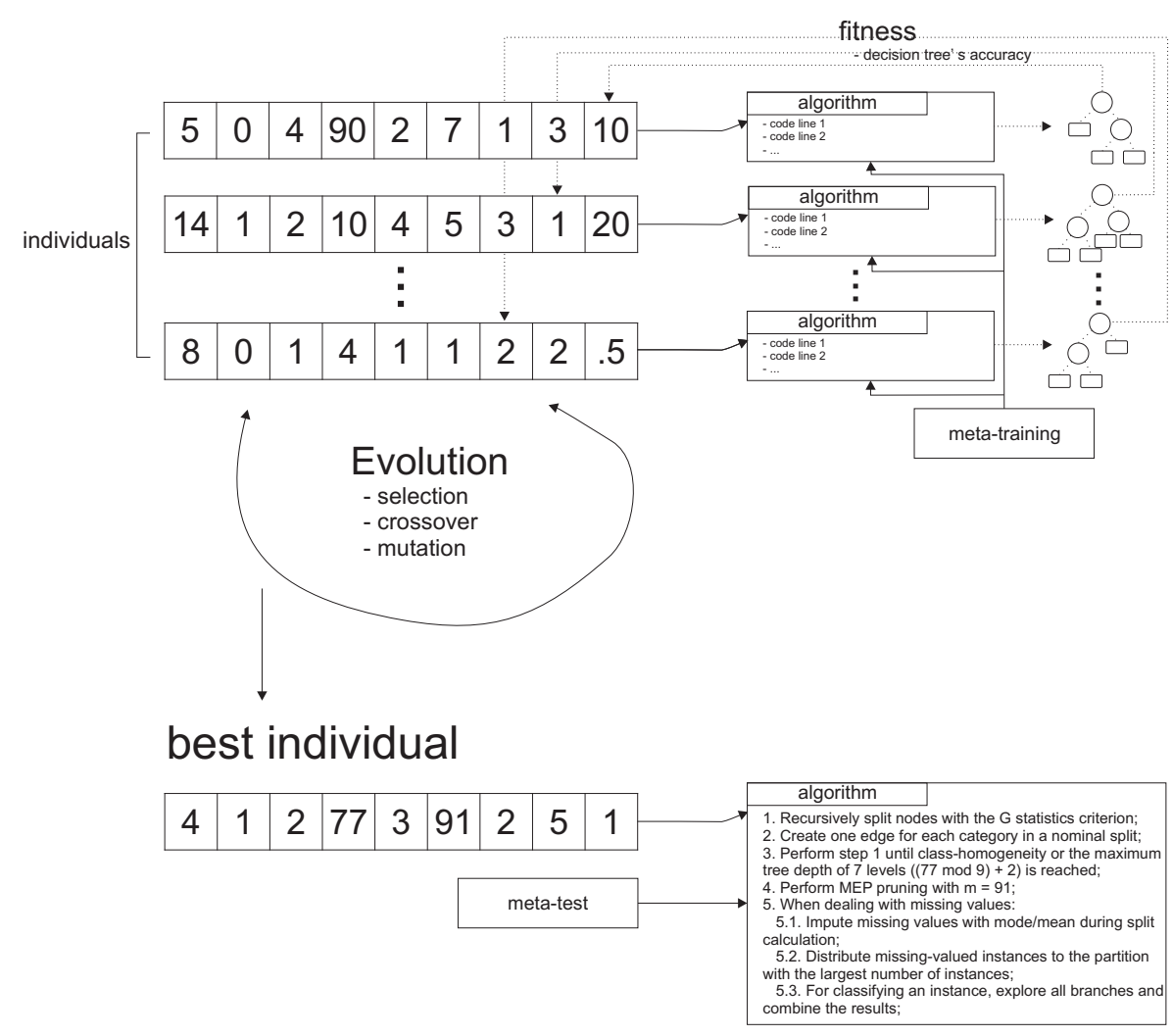

Figure 5.4: Evolution of individuals encoded as integer vectors.

\subsection{Fitness Evaluation}

During the fitness evaluation, HEAD-DT employs a meta-training set for assessing the quality of each individual throughout evolution. A meta-test set is used for assessing the quality of the evolved decision-tree induction algorithm (the best individual in Figure 5.1). There are two distinct frameworks for dealing with the meta-training and test sets: 


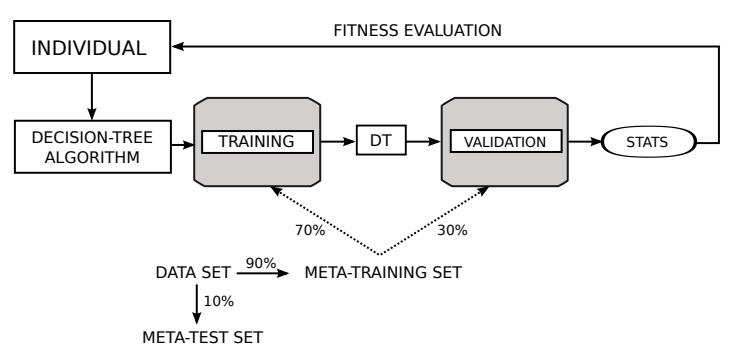

(a) Fitness evaluation from one data set in the meta-training set.

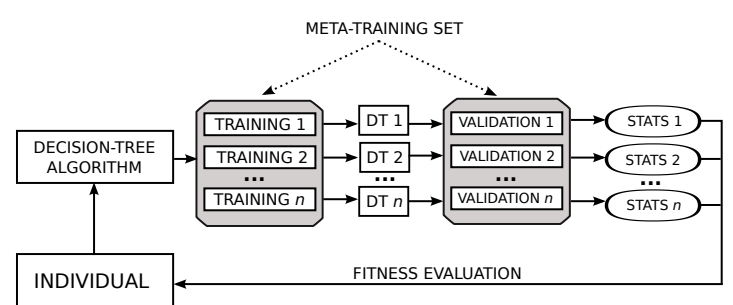

(b) Fitness evaluation from multiple data sets in the meta-training set.

Figure 5.5: Fitness evaluation schemes.

1. Evolving a decision-tree induction algorithm tailored to one specific data set.

2. Evolving a decision-tree induction algorithm from multiple data sets.

In the first case, named the specific framework, we have a specific data set for which we want to design a decision-tree algorithm. The meta-training set comprises the available training data from the data set at hand. The meta-test set comprises test data (belonging to the same data set) we have available for evaluating the performance of the algorithm (see Figure 5.5a). For example, suppose HEAD-DT is employed to evolve the near-optimal decision-tree induction algorithm for the iris data set. In such a scenario, both meta-training and meta-test sets comprise distinct data folds from the iris data set.

In the second case, named the general framework, there are multiple data sets composing the meta-training set, and possibly multiple (albeit different) data sets comprising the meta-test set (see Figure 5.5b). For example, suppose HEAD-DT is employed to evolve the near-optimal algorithm for the problem of credit risk assessment. In this scenario, the meta-training set may comprise public UCI data sets (Frank and Asuncion, 2010) such as german credit and credit approval, whereas the meta-test set may comprise particular credit risk assessment data sets the user desires to classify.

The general framework can be employed with two different objectives, broadly speaking:

1. Designing a decision-tree algorithm whose predictive performance is consistently good in a wide variety of data sets. For such, the evolved algorithm is applied to data sets with very different structural characteristics and/or from very distinct application domains. In this scenario, the user chooses distinct data sets to be part of the meta-training set, in the hope that evolution will be capable of generating an algorithm that performs well in a wide range of data sets. Pappa (2007) calls this strategy "evolving robust algorithms";

2. Designing a decision-tree algorithm that is tailored to a particular application domain or to a specific statistical profile. In this scenario, the meta-training set comprises data sets that share similarities, and so the evolved decision-tree algorithm is specialized in solving a specific type of problem. Unlike the previous strategy, in this case we have to define a 
similarity criterion for creating specialized algorithms. We highlight the following similarity criteria: (i) choosing data sets that share the same application domain (e.g., gene expression data); (ii) choosing data sets with provenance resemblance (e.g., data sets generated from data collected by a specific sensor); and (iii) choosing data sets with structural resemblance (e.g., data sets with statistically-similar features and/or with similar geometrical complexity (Ho and Basu, 2002; Ho et al., 2006)).

In Figure 5.5b, we can observe how the fitness evaluation of a decision-tree induction algorithm evolved from multiple data sets occurs. First, a given individual is mapped into its corresponding decision-tree algorithm. Afterwards, each data set that belongs to the meta-training set is divided into training and validation - typical values are $70 \%$ for training and $30 \%$ for validation (Witten and Frank, 1999). The term "validation set" is used in here instead of "test set" to avoid confusion with the meta-test set, and also due to the fact that we are using the "knowledge" within these sets to reach for a better solution (the same cannot be done with test sets, which are exclusively used for assessing the performance of an algorithm).

After dividing each data set from the meta-training set into "training" and "validation", a decision tree is induced for each training set available. For evaluating the performance of these decision trees, we use the corresponding validation sets. Statistics regarding the performance of each decision tree are recorded (e.g., accuracy, F-Measure, precision, recall, total number of nodes/leaves, etc.), and can be used individually or combined as the fitness function of HEAD-DT. The simple average is probably the most intuitive way of combining the values per data set, but other possible solutions are the median of the values, or their harmonic mean. Depending on the data sets used in the meta-training set, the user may decide to give greater weight of importance to a more difficult-to-solve data set than to an easier one, and hence a weighted scheme may be a good solution when combining the data set values. Some of these possibilities are discussed in Chapter 7.

A typical fitness function would be the average F-Measure of the decision trees generated by a given individual for each data set from the meta-training set. F-Measure (also known as F-score or F1 score) is the harmonic mean of precision and recall:

$$
\begin{gathered}
\text { precision }=\frac{t p}{t p+f p} \\
\text { recall }=\frac{t p}{t p+f n} \\
\text { fmeasure }=2 \times \frac{\text { precision } \times \text { recall }}{\text { precision }+ \text { recall }} \\
\text { Fitness }=\frac{1}{n} \sum_{i=1}^{n} \text { fmeasure }_{i}
\end{gathered}
$$


where $t p(t n)$ is the number of true positives (negatives), $f p(f n)$ is the number of false positives (negatives), fmeasure $_{i}$ is the F-Measure obtained in data set $i$ and $n$ is the total number of data sets in the meta-training set.

This formulation assumes that the classification problem at hand is binary, i.e., composed by two classes: positive and negative. Nevertheless, it can be trivially extended to multi-class problems. For instance, we can compute the measure for each class - assuming each class to be the "positive" class in turn - and (weight-)average the per-class measures. Having in mind that we would like HEAD-DT to perform well in both balanced and imbalanced data sets, we believe that the average F-Measure is a more suitable fitness function than the average accuracy.

\subsection{Search Space}

To compute the search space reached by HEAD-DT, consider the linear genome presented in Section 5.2: [split criterion, split type, stopping criterion, stopping parameter, pruning strategy, pruning parameter, $m v$ split, $m v$ distribution, mv classification]. There are 15 types of split criteria, 2 possible split types, 4 types of missing-value strategies during split computation, 7 types of missing-value strategies during training data distribution, and 3 types of missing-value strategies during classification. Hence, there are $15 \times 2 \times 4 \times 7 \times 3=2,520$ possible different algorithms.

Now, let us analyse the combination of stopping criteria and their parameters. There is the possibility of splitting until class homogeneity is achieved, and no parameter is needed (thus, 1 possible algorithm). There are 9 possible parameters when the tree is grown until a maximum depth, and 20 when reaching a minimum number of instances. Furthermore, there are 10 possible parameter values when reaching a minimum percentage of instances and 7 when reaching an accuracy threshold. Hence, there are $1+9+20+10+7=47$ possible algorithms just by varying the stopping criteria component.

Next, let us analyse the combination of pruning methods and their parameters. REP pruning parameter may take up to 5 different values, whereas PEP pruning may take up to 4 . MEP can take up to 101 values, and EBP up to 50. Finally, CCP takes up to 4 values for its first parameter and up to 5 values for its second. Therefore, there are $5+4+101+(4 \times 5)+50=180$ possible algorithms by just varying the pruning component.

If we combine all the previously mentioned values, HEAD-DT currently searches in the space of $2,520 \times 47 \times 180=21,319,200$ algorithms. Now, just for the sake of argument, suppose a single decision-tree induction algorithm takes about 10 seconds to produce a decision tree for a given (small) data set for which we want the best possible algorithm. If we were to try all possible algorithms in a brute-force approach, we would take 59,220 hours to find out the best possible configuration for that data set. That means $\approx 2,467$ days or 6.75 years just to find out the best decision-tree algorithm for a single (small) data set. HEAD-DT would take, in the worst case, 100,000 seconds - 10,000 individuals (100 individuals per generation, 100 generations) 
times 10 seconds. Thus HEAD-DT would take about 1,666 minutes (27.7 hours) to compute the (near-)optimal algorithm for that same data set, i.e., it is $\approx 2,138$ times faster than the brute-force approach. In practice, this number is much smaller considering that individuals are not re-evaluated if not changed, and HEAD-DT implements reproduction and elitism.

Of course there are no theoretic guarantees that the (near-)optimal algorithm found by HEAD-DT within these 27.7 hours is going to be the same global optimal algorithm provided by the brute-force approach after practically 7 years of computation, but its use is justified by the time saved during the process.

\subsection{Related Work}

The literature in EAs for decision-tree induction is very rich, as detailed in the survey presented in Chapter 4, and later published in (Barros et al., 2012a). However, as we point out in Section 4.7.3, the research community is still concerned with the evolution of decision trees for particular data sets instead of evolving full decision-tree induction algorithms.

To the best of our knowledge, no work to date attempts to automatically design full decision-tree induction algorithms. The most related approach to the one presented in this thesis is HHDT (Hyper-Heuristic Decision Tree) (Vella et al., 2010). It proposes an EA for evolving heuristic rules in order to determine the best splitting criterion to be used in non-terminal nodes. It is based on the degree of entropy of the data set attributes. For instance, it evolves rules such as $I F(x \% \geq$ high) and $(y \%<$ low) THEN use heuristic $A$, where $x$ and $y$ are are percentages ranging within $[0,100]$, and high and low are threshold entropy values. This rule has the following interpretation: if $x \%$ of the attributes have entropy values greater or equal than threshold high, and if $y \%$ of the attributes have entropy values below threshold low, then make use of heuristic $A$ for choosing the attribute that splits the current node. Whilst HHDT is a first step to automate the design of decision-tree induction algorithms, it evolves a single component of the algorithm (the choice of splitting criterion), and thus should be further extended for being able to generate full decision-tree induction algorithms, which is the case of HEAD-DT.

Another slightly related approach is the one presented by Delibasic et al. (2011). The authors propose a framework for combining decision-tree components, and test 80 different combination of design components on 15 benchmark data sets. This approach is not a hyper-heuristic, since it does not present an heuristic to choose among different heuristics. It simply selects a fixed number of component combinations and test them all against traditional decision-tree algorithms (C4.5, CART, ID3 and CHAID). We believe that employing EAs to evolve decision-tree algorithms is a more robust strategy, since we can search for solutions in a much larger search space (21 million possible algorithms in HEAD-DT, against 80 different algorithms in the work of Delibasic et al. (2011)). 
Finally, the work of Pappa (2007); Pappa and Freitas (2009a,b), which was the original inspiration for this thesis, proposes a grammar-based genetic programming approach (GGP) for evolving full rule induction algorithms. The results showed that GGP could generate rule induction algorithms different from those already proposed in the literature, and with competitive predictive performance.

\subsection{Chapter Remarks}

In this chapter, we presented HEAD-DT, a hyper-heuristic evolutionary algorithm that automatically designs top-down decision-tree induction algorithms. The latter have been manually improved for the last 40 years, resulting in a large number of approaches for each of their design components. Since the human manual approach for testing all possible modifications in the design components of decision-tree algorithms would be unfeasible, we believe the evolutionary search of HEAD-DT constitutes a robust and efficient solution for the problem.

HEAD-DT evolves individuals encoded as integer vectors (linear genome). Each gene in the vector is an index to a design component or the value of its corresponding parameter. Individuals are decoded by associating each integer to a design component, and by mapping values ranging within $[0,100]$ to values in the correct range according to the specified component. The initial population of 100 individuals evolve for 100 generations, in which individuals are chosen by a pairwise tournament selection strategy to participate of mutually-exclusive genetic operators such as uniform crossover, random uniform gene mutation, and reproduction.

HEAD-DT may operate under two distinct frameworks: i) evolving a decision-tree induction algorithm tailored to one specific data set; and ii) evolving a decision-tree induction algorithm from multiple data sets. In the first framework, the goal is to generate a decision-tree algorithm that excels at a single data set (both meta-training and meta-test sets comprise data from the same data set). In the second framework, there are several distinct objectives that can be achieved, like generating a decision-tree algorithm tailored to a particular application domain (say gene expression data sets or financial data sets), or generating a decision-tree algorithm that is robust across several different data sets (a good "all-around" algorithm).

Regardless of the framework being employed, HEAD-DT is capable of searching in a space of more than 21 million algorithms. In the next chapter, we present several experiments for evaluating HEAD-DT's performance under the two proposed frameworks. Moreover, we comment on the cost-effectiveness of automated algorithm design in contrast to the manual design, and we show that the genetic search performed by HEAD-DT is significantly better than a random search in the space of 21 million decision-tree induction algorithms. 


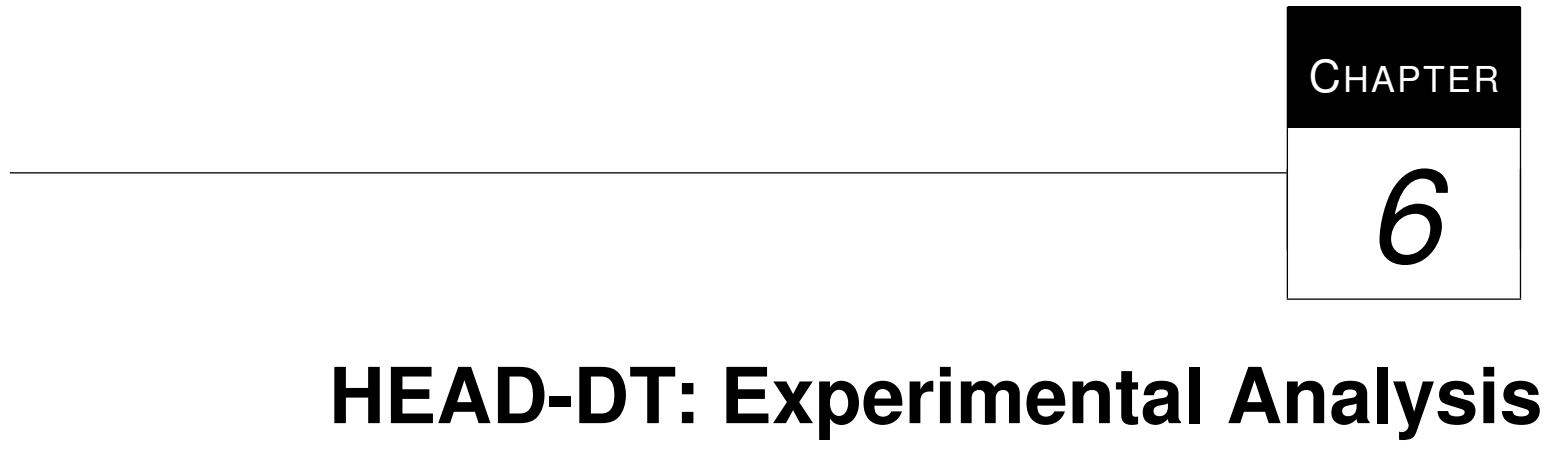

In this chapter, we present several empirical analyses that assess the performance of HEAD-DT in different scenarios. We divide these analyses into two sets of experiments, according to the meta-training strategy employed for automatically designing the decision-tree algorithms. As mentioned in Chapter 5, HEAD-DT can operate in two distinct frameworks: (i) evolving a decision-tree induction algorithm tailored to one specific data set (specific framework); or (ii) evolving a decision-tree induction algorithm from multiple data sets (general framework).

The specific framework provides data from a single data set to HEAD-DT for both algorithm design (evolution) and performance assessment. The experiments conducted for this scenario (see Section 6.1) make use of public data sets that do not share a common application domain. In the general framework, distinct data sets are used for algorithm design and performance assessment. In this scenario (see Section 6.2), we conduct two types of experiments, namely the homogeneous approach and the heterogeneous approach. In the homogeneous approach, we analyse whether automatically designing a decision-tree algorithm for a particular domain provides good results. More specifically, the data sets that feed HEAD-DT during evolution, and also those employed for performance assessment, share a common application domain. In the heterogeneous approach, we investigate whether HEAD-DT is capable of generating an algorithm that performs well across a variety of different data sets, regardless of their particular characteristics or application domain.

We also discuss about the theoretic and empirical time complexity of HEAD-DT in Section 6.3, and we make a brief discussion on the cost-effectiveness of automated algorithm design in Section 6.4. We present examples of algorithms which were automatically designed by HEAD-DT in Section 6.5. We conclude the experimental analysis by empirically verifying in Section 6.6 whether the genetic search is worthwhile. 


\subsection{Evolving algorithms tailored to one specific data set}

In this first set of experiments, we investigate the performance of HEAD-DT with regard to the specific framework. For that, we employed 20 public data sets (see Table 6.1) that were collected from the UCI machine-learning repository ${ }^{1}$ (Frank and Asuncion, 2010). We compare the resulting decision-tree algorithms with the two most well-known and widely-used decision-tree induction algorithms: C4.5 (Quinlan, 1993) and CART (Breiman et al., 1984). We report the classification accuracy of the decision trees generated for each data set, as well as the F-Measure, and the size of the decision tree (total number of nodes). All results are based on the average of 10-fold cross-validation runs. Additionally, since HEAD-DT is a non-deterministic method, we averaged the results of 5 different runs (varying the random seed).

Table 6.1: Summary of the data sets used in the experiments.

\begin{tabular}{|c|c|c|c|c|c|c|}
\hline data set & \# instances & \# attributes & \# numeric attributes & \# nominal attributes & $\%$ missing & \# classes \\
\hline abalone & 4177 & 8 & 7 & 1 & 0.00 & 30 \\
\hline anneal & 898 & 38 & 6 & 32 & 0.00 & 6 \\
\hline arrhythmia & 452 & 279 & 206 & 73 & 0.32 & 16 \\
\hline audiology & 226 & 69 & 0 & 69 & 2.03 & 24 \\
\hline bridges_version 1 & 107 & 12 & 3 & 9 & 5.53 & 6 \\
\hline car & 1728 & 6 & 0 & 6 & 0.00 & 4 \\
\hline cylinder_bands & 540 & 39 & 18 & 21 & 4.74 & 2 \\
\hline glass & 214 & 9 & 9 & 0 & 0.00 & 7 \\
\hline hepatitis & 155 & 19 & 6 & 13 & 5.67 & 2 \\
\hline iris & 150 & 4 & 4 & 0 & 0.00 & 3 \\
\hline kdd_synthetic & 600 & 61 & 60 & 1 & 0.00 & 6 \\
\hline segment & 2310 & 19 & 19 & 0 & 0.00 & 7 \\
\hline semeion & 1593 & 265 & 265 & 0 & 0.00 & 2 \\
\hline shuttle_landing & 15 & 6 & 0 & 6 & 28.89 & 2 \\
\hline sick & 3772 & 30 & 6 & 22 & 5.54 & 2 \\
\hline tempdiag & 120 & 7 & 1 & 6 & 0.00 & 2 \\
\hline tep.fea & 3572 & 7 & 7 & 0 & 0.00 & 3 \\
\hline vowel & 990 & 13 & 10 & 3 & 0.00 & 11 \\
\hline winequality_red & 1599 & 11 & 11 & 0 & 0.00 & 10 \\
\hline winequality_white & 4898 & 11 & 11 & 0 & 0.00 & 10 \\
\hline
\end{tabular}

The baseline algorithms are configured with default parameters. We did not perform a parameter optimisation procedure in this set of experiments, given that we are designing one algorithm per data set, and optimising a set of parameters for each data set is not feasible. Thus, we employed typical values found in the literature of evolutionary algorithms for decision-tree induction (see Chapter 4):

- Fitness-function: F-Measure;

- Population size: 100;

- Maximum number of generations: 100;

- Selection: tournament selection with size $t=2$;

${ }^{1}$ http://archive.ics.uci.edu/ml/ 
- Elitism rate: 5 individuals;

- Crossover: uniform crossover with $90 \%$ probability;

- Mutation: random uniform gene mutation with 5\% probability;

- Reproduction: cloning individuals with 5\% probability.

In order to provide some reassurance about the validity and non-randomness of the obtained results, we present the results of statistical tests by following the approach proposed by Demšar (2006). In brief, this approach seeks to compare multiple algorithms on multiple data sets, and it is based on the use of the Friedman test with a corresponding post-hoc test. The Friedman test is a non-parametric counterpart of the well-known ANOVA, as follows. Let $R_{i}^{j}$ be the rank of the $j^{\text {th }}$ of $k$ algorithms on the $i^{t h}$ of $N$ data sets. The Friedman test compares the average ranks of algorithms, $R_{j}=\frac{1}{N} \sum_{i} R_{i}^{j}$. The Friedman statistic is given by:

$$
\chi_{F}^{2}=\frac{12 N}{k(k+1)}\left[\sum_{j} R_{j}^{2}-\frac{k(k+1)^{2}}{4}\right],
$$

and it is distributed according to $\chi_{F}^{2}$ with $k-1$ degrees of freedom, when $N$ and $k$ are big enough.

Iman and Davenport (1980) showed that Friedman's $\chi_{F}^{2}$ is undesirably conservative and derived an adjusted statistic:

$$
F_{f}=\frac{(N-1) \times \chi_{F}^{2}}{N \times(k-1)-\chi_{F}^{2}}
$$

which is distributed according to the $F$-distribution with $k-1$ and $(k-1)(N-1)$ degrees of freedom.

If the null hypothesis of similar performances is rejected, then we proceed with the Nemenyi post-hoc test for pairwise comparisons. The performance of two classifiers is significantly different if their corresponding average ranks differ by at least the critical difference

$$
C D=q_{\alpha} \sqrt{\frac{k(k+1)}{6 N}}
$$

where critical values $q_{\alpha}$ are based on the Studentized range statistic divided by $\sqrt{2}$.

Table 6.2 shows the classification accuracy of C4.5, CART, and HEAD-DT. It illustrates the average accuracy over the 10-fold cross-validation runs \pm the standard deviation of the accuracy obtained in those runs (best absolute values in bold). It is possible to see that HEAD-DT generates more accurate trees in 13 out of the 20 data sets. CART provides more accurate trees in two data sets, and C4.5 in none. In the remaining 5 data sets, no method was superior to the others.

To evaluate the statistical significance of the accuracy results, we calculated the average rank for CART, C4.5 and HEAD-DT: 2.375, 2.2 and 1.425, respectively. The average rank suggests that 
Table 6.2: Classification accuracy of CART, C4.5 and HEAD-DT.

\begin{tabular}{lccc}
\hline & CART & C4.5 & HEAD-DT \\
\hline abalone & $\mathbf{0 . 2 6} \pm \mathbf{0 . 0 2}$ & $0.22 \pm 0.02$ & $0.20 \pm 0.02$ \\
anneal & $0.98 \pm 0.01$ & $0.99 \pm 0.01$ & $0.99 \pm 0.01$ \\
arrhythmia & $\mathbf{0 . 7 1} \pm \mathbf{0 . 0 5}$ & $0.65 \pm 0.04$ & $0.65 \pm 0.04$ \\
audiology & $0.74 \pm 0.05$ & $0.78 \pm 0.07$ & $\mathbf{0 . 8 0} \pm \mathbf{0 . 0 6}$ \\
bridges_version1 & $0.41 \pm 0.07$ & $0.57 \pm 0.10$ & $\mathbf{0 . 6 0} \pm \mathbf{0 . 1 2}$ \\
car & $0.97 \pm 0.02$ & $0.93 \pm 0.02$ & $\mathbf{0 . 9 8} \pm \mathbf{0 . 0 1}$ \\
cylinder_bands & $0.60 \pm 0.05$ & $0.58 \pm 0.01$ & $\mathbf{0 . 7 2} \pm \mathbf{0 . 0 4}$ \\
glass & $0.70 \pm 0.11$ & $0.69 \pm 0.04$ & $\mathbf{0 . 7 3} \pm \mathbf{0 . 1 0}$ \\
hepatitis & $0.79 \pm 0.05$ & $0.79 \pm 0.06$ & $\mathbf{0 . 8 1} \pm \mathbf{0 . 0 8}$ \\
iris & $0.93 \pm 0.05$ & $0.94 \pm 0.07$ & $\mathbf{0 . 9 5} \pm \mathbf{0 . 0 4}$ \\
kdd_synthetic & $0.88 \pm 0.00$ & $0.91 \pm 0.04$ & $\mathbf{0 . 9 7} \pm \mathbf{0 . 0 3}$ \\
segment & $0.96 \pm 0.01$ & $0.97 \pm 0.01$ & $0.97 \pm 0.01$ \\
semeion & $0.94 \pm 0.01$ & $0.95 \pm 0.02$ & $\mathbf{1 . 0 0} \pm \mathbf{0 . 0 0}$ \\
shuttle_landing & $0.95 \pm 0.16$ & $0.95 \pm 0.16$ & $\mathbf{0 . 9 5} \pm \mathbf{0 . 1 5}$ \\
sick & $0.99 \pm 0.01$ & $0.99 \pm 0.00$ & $0.99 \pm 0.00$ \\
tempdiag & $1.00 \pm 0.00$ & $1.00 \pm 0.00$ & $1.00 \pm 0.00$ \\
tep.fea & $0.65 \pm 0.02$ & $0.65 \pm 0.02$ & $0.65 \pm 0.02$ \\
vowel & $0.82 \pm 0.04$ & $0.83 \pm 0.03$ & $\mathbf{0 . 8 9} \pm \mathbf{0 . 0 4}$ \\
winequality_red & $0.63 \pm 0.02$ & $0.61 \pm 0.03$ & $\mathbf{0 . 6 4} \pm \mathbf{0 . 0 3}$ \\
winequality_white & $0.58 \pm 0.02$ & $0.61 \pm 0.03$ & $\mathbf{0 . 6 3} \pm \mathbf{0 . 0 3}$ \\
\hline
\end{tabular}

HEAD-DT is the best performing method regarding accuracy. The calculation of Friedman's $\chi_{F}^{2}$ is given by:

$$
\chi_{F}^{2}=\frac{12 \times 20}{3 \times 4}\left[2.375^{2}+2.2^{2}+1.425^{2}-\frac{3 \times 4^{2}}{4}\right]=10.225
$$

Iman's $F$ statistic is given by:

$$
F_{f}=\frac{(20-1) \times 10.225}{20 \times(3-1)-10.225}=6.52
$$

Critical value of $F(k-1,(k-1)(n-1))=F(2,38)$ for $\alpha=0.05$ is 3.25. Since $F_{f}>$ $F_{0.05}(2,38)(6.52>3.25)$, the null-hypothesis is rejected. We proceed with a post-hoc Nemenyi test to find which method provides better results. The critical difference $C D$ is given by:

$$
C D=2.343 \times \sqrt{\frac{3 \times 4}{6 \times 20}}=0.74
$$

The difference between the average rank of HEAD-DT and C4.5 is 0.775 , and between HEAD-DT and CART is 0.95 . Since both the differences are greater than $C D$, the performance of HEAD-DT is significantly better than both C4.5 and CART regarding accuracy.

Table 6.3 shows the classification F-Measure of C4.5, CART and HEAD-DT. The experimental results show that HEAD-DT generates better trees (regardless of the class imbalance problem) in 16 out of the 20 data sets. CART generates the best tree in two data sets, while C4.5 does not provide the best tree for any data set.

We calculated the average rank for CART, C4.5 and HEAD-DT: 2.5, 2.225 and 1.275, respectively. The average rank suggest that HEAD-DT is the best performing method regarding F-Measure. The calculation of Friedman's $\chi_{F}^{2}$ is given by: 
Table 6.3: Classification F-Measure of CART, C4.5 and HEAD-DT.

\begin{tabular}{lccc}
\hline & CART & C4.5 & HEAD-DT \\
\hline abalone & $\mathbf{0 . 2 2} \pm \mathbf{0 . 0 2}$ & $0.21 \pm 0.02$ & $0.20 \pm 0.02$ \\
anneal & $0.98 \pm 0.01$ & $0.98 \pm 0.01$ & $\mathbf{0 . 9 9} \pm \mathbf{0 . 0 1}$ \\
arrhythmia & $\mathbf{0 . 6 7} \pm \mathbf{0 . 0 5}$ & $0.64 \pm 0.05$ & $0.63 \pm 0.06$ \\
audiology & $0.70 \pm 0.04$ & $0.75 \pm 0.08$ & $\mathbf{0 . 7 9} \pm \mathbf{0 . 0 7}$ \\
bridges_version1 & $0.44 \pm 0.06$ & $0.52 \pm 0.10$ & $\mathbf{0 . 5 6} \pm \mathbf{0 . 1 2}$ \\
car & $0.93 \pm 0.97$ & $0.93 \pm 0.02$ & $\mathbf{0 . 9 8} \pm \mathbf{0 . 0 1}$ \\
cylinder_bands & $0.54 \pm 0.07$ & $0.42 \pm 0.00$ & $\mathbf{0 . 7 2} \pm \mathbf{0 . 0 4}$ \\
glass & $0.67 \pm 0.10$ & $0.67 \pm 0.05$ & $\mathbf{0 . 7 2} \pm \mathbf{0 . 0 9}$ \\
hepatitis & $0.74 \pm 0.07$ & $0.77 \pm 0.06$ & $\mathbf{0 . 8 0} \pm \mathbf{0 . 0 8}$ \\
iris & $0.93 \pm 0.05$ & $0.93 \pm 0.06$ & $\mathbf{0 . 9 5} \pm \mathbf{0 . 0 5}$ \\
kdd_synthetic & $0.88 \pm 0.03$ & $0.90 \pm 0.04$ & $\mathbf{0 . 9 7} \pm \mathbf{0 . 0 3}$ \\
segment & $0.95 \pm 0.01$ & $0.96 \pm 0.09$ & $\mathbf{0 . 9 7} \pm \mathbf{0 . 0 1}$ \\
semeion & $0.93 \pm 0.01$ & $0.95 \pm 0.02$ & $\mathbf{1 . 0 0} \pm \mathbf{0 . 0 0}$ \\
shuttle_landing & $0.56 \pm 0.03$ & $0.56 \pm 0.38$ & $\mathbf{0 . 9 3} \pm \mathbf{0 . 2 0}$ \\
sick & $0.98 \pm 0.00$ & $0.98 \pm 0.00$ & $\mathbf{0 . 9 9} \pm \mathbf{0 . 0 0}$ \\
tempdiag & $1.00 \pm 0.00$ & $1.00 \pm 0.00$ & $1.00 \pm 0.00$ \\
tep.fea & $0.60 \pm 0.02$ & $0.61 \pm 0.02$ & $0.61 \pm 0.02$ \\
vowel & $0.81 \pm 0.03$ & $0.82 \pm 0.03$ & $\mathbf{0 . 8 9} \pm \mathbf{0 . 0 3}$ \\
winequality_red & $0.61 \pm 0.02$ & $0.60 \pm 0.03$ & $\mathbf{0 . 6 3} \pm \mathbf{0 . 0 3}$ \\
winequality_white & $0.57 \pm 0.02$ & $0.60 \pm 0.02$ & $\mathbf{0 . 6 3} \pm \mathbf{0 . 0 3}$ \\
\hline
\end{tabular}

$$
\chi_{F}^{2}=\frac{12 \times 20}{3 \times 4}\left[2.5^{2}+2.225^{2}+1.275^{2}-\frac{3 \times 4^{2}}{4}\right]=16.525
$$

Table 6.4: Tree size of CART, C4.5 and HEAD-DT trees.

\begin{tabular}{lrrr}
\hline & \multicolumn{1}{c}{ CART } & \multicolumn{1}{c}{ C4.5 } & HEAD-DT \\
\hline abalone & $\mathbf{4 4 . 4 0} \pm \mathbf{1 6 . 0 0}$ & $2088.90 \pm 37.63$ & $4068.12 \pm 13.90$ \\
anneal & $\mathbf{2 1 . 0 0} \pm \mathbf{3 . 1 3}$ & $48.30 \pm 6.48$ & $55.72 \pm 3.66$ \\
arrhythmia & $\mathbf{2 3 . 2 0} \pm \mathbf{2 . 9 0}$ & $82.60 \pm 5.80$ & $171.84 \pm 5.18$ \\
audiology & $\mathbf{3 5 . 8 0} \pm \mathbf{1 1 . 7 5}$ & $50.40 \pm 4.01$ & $118.60 \pm 3.81$ \\
bridges_version1 & $\mathbf{1 . 0 0} \pm \mathbf{0 . 0 0}$ & $24.90 \pm 20.72$ & $156.88 \pm 14.34$ \\
car & $\mathbf{1 0 8 . 2 0} \pm \mathbf{1 6 . 0 9}$ & $173.10 \pm 6.51$ & $171.92 \pm 4.45$ \\
cylinder_bands & $4.20 \pm 1.03$ & $\mathbf{1 . 0 0} \pm \mathbf{0 . 0 0}$ & $211.44 \pm 9.39$ \\
glass & $\mathbf{2 3 . 2 0} \pm \mathbf{1 0 . 5 6}$ & $44.80 \pm 5.20$ & $86.44 \pm 3.14$ \\
hepatitis & $\mathbf{6 . 6 0} \pm \mathbf{8 . 5 8}$ & $15.40 \pm 4.40$ & $71.80 \pm 4.77$ \\
iris & $\mathbf{6 . 2 0} \pm \mathbf{1 . 6 9}$ & $8.00 \pm 1.41$ & $20.36 \pm 1.81$ \\
kdd_synthetic & $\mathbf{1 . 0 0} \pm \mathbf{0 . 0 0}$ & $37.80 \pm 4.34$ & $26.16 \pm 2.45$ \\
segment & $\mathbf{7 8 . 0 0} \pm \mathbf{8 . 1 8}$ & $80.60 \pm 4.97$ & $132.76 \pm 3.48$ \\
semeion & $34.00 \pm 12.30$ & $55.00 \pm 8.27$ & $\mathbf{1 9 . 0 0} \pm \mathbf{0 . 0 0}$ \\
shuttle_landing & $1.00 \pm 0.00$ & $1.00 \pm 0.00$ & $5.64 \pm 1.69$ \\
sick & $\mathbf{4 5 . 2 0} \pm \mathbf{1 1 . 3 3}$ & $46.90 \pm 9.41$ & $153.70 \pm 8.89$ \\
tempdiag & $5.00 \pm 0.00$ & $5.00 \pm 0.00$ & $\mathbf{8 . 2 0} \pm \mathbf{1 . 6 9}$ \\
tep.fea & $13.00 \pm 2.83$ & $220.70 \pm 20.73$ & $18.84 \pm 1.97$ \\
vowel & $\mathbf{1 7 5 . 8 0} \pm \mathbf{2 3 . 7 2}$ & $387.00 \pm 26.55$ & $361.42 \pm 5.54$ \\
winequality_red & $\mathbf{1 5 1 . 8 0} \pm \mathbf{5 4 . 5 8}$ & $1367.20 \pm 58.44$ & $796.00 \pm 11.22$ \\
winequality_white & $\mathbf{8 4 3 . 8 0} \pm \mathbf{3 0 9 . 0 1}$ & & $2525.88 \pm 13.17$ \\
\hline
\end{tabular}

Iman's $F$ statistic is given by:

$$
F_{f}=\frac{(20-1) \times 16.525}{20 \times(3-1)-16.525}=13.375
$$

Since $F_{f}>F_{0.05}(2,38)(13.375>3.25)$, the null-hypothesis is rejected. The difference between the average rank of HEAD-DT and C4.5 is 0.95 and that between HEAD-DT and CART is 1.225 . Since both the differences are greater than $C D(0.74)$, the performance of HEAD-DT is significantly better than both $\mathrm{C} 4.5$ and CART regarding F-Measure. 
Table 6.4 shows the size of trees generated by C4.5, CART and HEAD-DT. Results show that CART generates smaller trees in 15 out of the 20 data sets. C4.5 generates smaller trees in 2 data sets, and HEAD-DT in only one data set. The statistical analysis is given as follows:

$$
\begin{gathered}
\chi_{F}^{2}=\frac{12 \times 20}{3 \times 4}\left[1.2^{2}+2^{2}+2.8^{2}-\frac{3 \times 4^{2}}{4}\right]=25.6 \\
F_{f}=\frac{(20-1) \times 33.78}{20 \times(3-1)-25.6}=33.78
\end{gathered}
$$

Since $F_{f}>F_{0.05}(2,38)(33.78>3.25)$, the null-hypothesis is rejected. The difference between the average rank of HEAD-DT and C4.5 is 0.8 and that between HEAD-DT and CART is 1.6. Since both the differences are greater than $C D(0.74)$, HEAD-DT generates trees which are significantly larger than both $\mathrm{C} 4.5$ and CART. This should not be a concern, since smaller trees are only preferable in scenarios where the predictive performance of the algorithms is similar.

The statistical analysis previously presented clearly indicates that HEAD-DT generates algorithms whose trees outperform C4.5 and CART regarding predictive performance. The Occam's razor assumption that among competitive hypotheses, the simpler is preferred, does not apply to this case.

\subsection{Evolving algorithms from multiple data sets}

In this section, we evaluate the performance of HEAD-DT when evolving a single algorithm from multiple data sets (the general framework). More specifically, we divide this experiment into two scenarios: (i) evolving a single decision-tree algorithm for data sets from a particular application domain (homogeneous approach); and (ii) evolving a single decision-tree algorithm for a variety of data sets (heterogeneous approach).

In both cases, we need to establish the methodology to select the data sets that will compose the meta-training and meta-test sets. Hence, we developed the following selection methodology: randomly choose 1 data set from the available set to be part of the meta-training set; then, execute HEAD-DT with the selected data set in the meta-training set and the remaining data sets in the meta-test set; for the next experiment, select two additional data sets that were previously part of the meta-test set, and move them to the meta-training set, that now comprises 3 data sets. This procedure is repeated until we have 9 data sets being part of the meta-training set.

Considering that HEAD-DT is a regular generational EA (as depicted in Figure 5.1), the following parameters have to be chosen prior to evolution: (i) population size; (ii) maximum number of generations; (iii) tournament selection size; (iv) elitism rate; (v) reproduction probability; (vi) crossover probability; and (vii) mutation probability.

For parameters (i) - (iv), we defined values commonly used in the literature of evolutionary algorithms for decision-tree induction (Barros et al., 2011a), namely: 100 individuals, 100 
generations, tournament between 2 individuals, 5\% of elitism. For the remaining parameters, since the selected individuals will undergo either reproduction, crossover or mutation (mutually exclusive operators), we employ the single parameter $p$ that was previously presented in Chapter 5 , Section 5.3.

For both homogeneous and heterogeneous approaches, we performed a tuning procedure varying $p$ within $\{0.1,0.2,0.3,0.4,0.5,0.6,0.7,0.8,0.9\}$. In this tuning procedure, we employed a particular set of data sets to be part of the parameter optimisation group in order to evaluate the "optimal" value of $p$. Note that the aim of the parameter optimisation procedure is not to optimise the parameters for a particular data set, but to find robust values that work well across the tuning data sets. We then use the robust value of $p$ found in the procedure in another set of data sets that were selected to be part of the experiments. This evaluates the generalisation ability of $p$ across new data sets, unused for parameter tuning, as usual in supervised machine learning.

Also for both approaches, the fitness function employed by HEAD-DT is the average F-Measure of the data sets belonging to the meta-training set. To evaluate the performance of the best algorithm evolved by HEAD-DT, we performed a 10-fold cross-validation procedure for each data set belonging to the meta-test set, recording the accuracy and F-Measure achieved by each of the corresponding decision trees. Additionally, to mitigate the randomness effect of evolutionary algorithms, we average the results of 5 different runs of HEAD-DT.

In this framework, we once again make use of CART and C4.5 as the baseline algorithms in the experimental analysis. We employ their java versions available from the Weka machine learning toolkit (Witten and Frank, 1999) under the names of SimpleCART and J48. Moreover, we also compare HEAD-DT with the REPTree algorithm, which is a variation of C4.5 that employs reduced-error pruning, also available from the Weka toolkit.

For all baseline algorithms, we employ their default parameters, since they were carefully optimised by their respective authors throughout several years. Note that, in the supervised machine learning literature, the common approach is to find the optimal parameters for being used in a variety of distinct data sets, instead of optimising the algorithm for each specific data set.

\subsubsection{The Homogeneous Approach}

In this set of experiments, we assess the relative performance of the algorithm automatically designed by HEAD-DT for data sets from a particular application domain. More specifically, we make use of 35 publicly-available data sets from microarray gene expression data, described in (Souto et al., 2008). In brief, microarray technology enables expression level measurement for thousands of genes in parallel, given a biological tissue of interest. Once combined, results from a set of microarray experiments produce a gene expression data set. The data sets employed here are related to different types or subtypes of cancer, e.g., patients with prostate, lung, skin, and other types of cancer. The classification task refers to labeling different examples (instances) according 
to their gene (attribute) expression levels. The main structural characteristics of the 35 datasets are summarized in Table 6.5.

Table 6.5: Summary of the 35 Gene Expression data sets. For each data set, we present type of microarray chip, the total number of instances, total number of attributes, imbalanced ratio (rate between over- and under-represented class), and total number of classes.

\begin{tabular}{|c|c|c|c|c|c|c|}
\hline & $\begin{array}{c}\text { Data } \\
\text { Set }\end{array}$ & Chip & $\begin{array}{c}\# \\
\text { Instances }\end{array}$ & $\begin{array}{c}\# \\
\text { Attributes }\end{array}$ & IR & $\begin{array}{c}\# \\
\text { Classes }\end{array}$ \\
\hline & armstrong-v2 & Affy & 72 & 2193 & 1.40 & 3 \\
\hline & bredel & cDNA & 50 & 1738 & 6.20 & 3 \\
\hline & dyrskjot & Affy & 40 & 1202 & 2.22 & 3 \\
\hline & garber & cDNA & 66 & 4552 & 10.00 & 4 \\
\hline & golub-v2 & Affy & 72 & 1867 & 4.22 & 3 \\
\hline & gordon & Affy & 181 & 1625 & 4.84 & 2 \\
\hline Parameter & khan & cDNA & 83 & 1068 & 2.64 & 4 \\
\hline \multirow{17}{*}{ Optimisation } & laiho & Affy & 37 & 2201 & 3.63 & 2 \\
\hline & pomeroy-v2 & Affy & 42 & 1378 & 2.50 & 5 \\
\hline & ramaswamy & Affy & 190 & 1362 & 3.00 & 14 \\
\hline & su & Affy & 174 & 1570 & 4.67 & 10 \\
\hline & tomlins-v2 & cDNA & 92 & 1287 & 2.46 & 4 \\
\hline & yeoh-v1 & Affy & 248 & 2525 & 4.77 & 2 \\
\hline & yeoh-v2 & Affy & 248 & 2525 & 5.27 & 6 \\
\hline & alizadeh-v1 & cDNA & 42 & 1094 & 1.00 & 2 \\
\hline & alizadeh-v2 & cDNA & 62 & 2092 & 4.67 & 3 \\
\hline & alizadeh-v3 & cDNA & 62 & 2092 & 2.33 & 4 \\
\hline & armstrong-v1 & Affy & 72 & 1080 & 2.00 & 2 \\
\hline & bhattacharjee & Affy & 203 & 1542 & 23.17 & 5 \\
\hline & bittner & cDNA & 38 & 2200 & 1.00 & 2 \\
\hline & chen & cDNA & 179 & 84 & 1.39 & 2 \\
\hline & chowdary & Affy & 104 & 181 & 1.48 & 2 \\
\hline & golub-v1 & Affy & 72 & 1867 & 1.88 & 2 \\
\hline & lapointe-v1 & cDNA & 69 & 1624 & 3.55 & 3 \\
\hline \multirow[t]{11}{*}{ Experiments } & lapointe-v2 & cDNA & 110 & 2495 & 3.73 & 4 \\
\hline & liang & cDNA & 37 & 1410 & 9.34 & 3 \\
\hline & nutt-v1 & Affy & 50 & 1376 & 2.14 & 4 \\
\hline & nutt-v2 & Affy & 28 & 1069 & 1.00 & 2 \\
\hline & nutt-v3 & Affy & 22 & 1151 & 2.14 & 2 \\
\hline & shipp-v1 & Affy & 77 & 797 & 3.05 & 2 \\
\hline & pomeroy-v1 & Affy & 34 & 856 & 2.78 & 2 \\
\hline & risinger & cDNA & 42 & 1770 & 6.33 & 4 \\
\hline & singh & Affy & 102 & 338 & 1.04 & 2 \\
\hline & tomlins-v1 & cDNA & 104 & 2314 & 2.67 & 5 \\
\hline & west & Affy & 49 & 1197 & 1.04 & 2 \\
\hline
\end{tabular}

It is important to point out that microarray technology is generally available in two different types of platforms: single-channel microarrays (e.g., Affymetrix) or double-channel microarrays (e.g., cDNA). The type of microarray chip from each data set is in the second column of Table 6.5. Measurements of Affymetrix arrays are estimates on the number of RNA copies found in the cell sample, whereas cDNA microarrays values are ratios of the number of copies in relation to a control cell sample. As in (Souto et al., 2008), all genes with expression level below 10 are set to a minimum threshold of 10 in the Affymetrix data. The maximum threshold is set to 16,000 . This is because values below or above these thresholds are often said to be unreliable (Monti et al., 2003). Thus, the experimental analysis is performed on the scaled data to which the ceiling and threshold values have been applied. Still for the case of Affymetrix data, the following procedure is applied in order to remove uninformative genes: for each gene $j$ (attribute), compute the mean $m_{j}$ among the samples (instances). In order to get rid of extreme values, the first $10 \%$ largest and smallest values are discarded. Based on such a mean, every value $x_{i j}$ of gene $i$ and sample $j$ is transformed as follows: $y_{i j}=\log _{2}\left(x_{i j} / m_{j}\right)$. We then selected genes with expression levels differing by at least $l$-fold in at least $c$ samples from their mean expression level across the samples. With few exceptions, the parameters $l$ and $c$ were selected in order to produce a filtered data set with at least 
$10 \%$ of the original number of genes ${ }^{2}$. It should be noticed that the transformed data is only used in the filtering step. A similar filtering procedure was applied for the cDNA data, but without the log transformation. In the case of cDNA microarray data sets, genes with more than $10 \%$ of missing values were discarded. The remaining genes that still presented missing values had them replaced by its respective mean value.

Note that we randomly divided the 35 data sets into two groups: parameter optimisation and experiments. The 14 data sets in the parameter optimisation group are used for tuning the evolutionary parameters of HEAD-DT. The remaining 21 data sets from the experiments group are used for evaluating the performance of the algorithms automatically designed by HEAD-DT.

Following the selection methodology previously presented, the 14 parameter optimisation data sets are arranged in 5 different experimental configurations \{\#training sets, \#test sets $\}:\{1 \times 13\}$, $\{3 \times 11\},\{5 \times 9\},\{7 \times 7\}$, and $\{9 \times 5\}$. Similarly, the 21 data sets in the experiments group are arranged in also 5 different experimental configurations $\{\#$ training sets, \#test sets $\}:\{1 \times 20\}$, $\{3 \times 18\},\{5 \times 16\},\{7 \times 14\}$, and $\{9 \times 12\}$. Table 6.6 presents the randomly selected data sets according to the configurations detailed above.

Table 6.6: Meta-training and meta-test configurations for the gene expression data. Data sets were randomly selected according to the methodology presented in the beginning of Section 6.2.

\begin{tabular}{|c|c|c|c|c|c|c|c|c|c|}
\hline \multicolumn{10}{|c|}{ Data sets for parameter optimisation } \\
\hline \multicolumn{2}{|c|}{$1 \times 13$} & \multicolumn{2}{|c|}{$3 \times 11$} & \multicolumn{2}{|c|}{$5 \times 9$} & \multicolumn{2}{|c|}{$7 \times 7$} & \multicolumn{2}{|c|}{$9 \times 5$} \\
\hline Meta-Training & Meta-Test & Meta-Training & Meta-Test & Meta-Training & Meta-Test & Meta-Training & Meta-Test & Meta-Training & Meta-Test \\
\hline bredel & $\begin{array}{l}\text { armstrong-v2 } \\
\text { dyrskjot } \\
\text { garber } \\
\text { golub-v2 } \\
\text { gordon } \\
\text { khan } \\
\text { laiho } \\
\text { pomeroy-v2 } \\
\text { ramaswamy } \\
\text { su } \\
\text { tomlins-v2 } \\
\text { yeoh-v1 } \\
\text { yeoh-v2 }\end{array}$ & $\begin{array}{r}\text { bredel } \\
\text { pomeroy-v2 } \\
\text { yeoh-v2 }\end{array}$ & $\begin{array}{l}\text { armstrong-v2 } \\
\text { dyrskjot } \\
\text { garber } \\
\text { golub-v2 } \\
\text { gordon } \\
\text { khan } \\
\text { laiho } \\
\text { ramaswamy } \\
\text { su } \\
\text { tomlins-v2 } \\
\text { yeoh-v1 }\end{array}$ & $\begin{array}{r}\text { bredel } \\
\text { pomeroy-v2 } \\
\text { yeoh-v2 } \\
\text { gordon } \\
\text { su }\end{array}$ & $\begin{array}{l}\text { armstrong-v2 } \\
\text { dyrskjot } \\
\text { garber } \\
\text { golub-v2 } \\
\text { khan } \\
\text { laiho } \\
\text { ramaswamy } \\
\text { tomlins-v2 } \\
\text { yeoh-v1 }\end{array}$ & $\begin{array}{r}\text { bredel } \\
\text { pomeroy-v2 } \\
\text { yeoh-v2 } \\
\text { gordon } \\
\text { su } \\
\text { armstrong-v2 } \\
\text { laiho }\end{array}$ & $\begin{array}{l}\text { dyrskjot } \\
\text { garber } \\
\text { golub-v2 } \\
\text { khan } \\
\text { ramaswamy } \\
\text { tomlins-v2 } \\
\text { yeoh-v1 }\end{array}$ & $\begin{array}{r}\text { bredel } \\
\text { pomeroy-v2 } \\
\text { yeoh-v2 } \\
\text { gordon } \\
\text { su } \\
\text { armstrong-v2 } \\
\text { laiho } \\
\text { khan } \\
\text { tomlins-v2 }\end{array}$ & $\begin{array}{l}\text { dyrskjot } \\
\text { garber } \\
\text { golub-v2 } \\
\text { ramaswamy } \\
\text { yeoh-v1 }\end{array}$ \\
\hline \multirow{2}{*}{\multicolumn{4}{|c|}{$1 \times 20$}} & \multicolumn{6}{|c|}{ Data sets for the experimental analysis } \\
\hline & & & & $\{5, x y$ & & & & & \\
\hline Meta-Training & Meta-Test & Meta-Training & Meta-Test & Meta-Training & Meta-Test & Meta-Training & Meta-Test & Meta-Training & Meta-Test \\
\hline nutt-v3 & $\begin{array}{l}\text { alizadeh-v1 } \\
\text { alizadeh-v2 } \\
\text { alizadeh-v3 } \\
\text { armstrong-v1 } \\
\text { bhattacharjee } \\
\text { bittner } \\
\text { chen } \\
\text { chowdary } \\
\text { golub-v1 } \\
\text { lapointe-v1 } \\
\text { lapointe-v2 } \\
\text { liang } \\
\text { nutt-v1 } \\
\text { nutt-v2 } \\
\text { shipp-v1 } \\
\text { pomeroy-v1 } \\
\text { risinger } \\
\text { singh } \\
\text { tomlins-v1 } \\
\text { west }\end{array}$ & $\begin{array}{r}\text { nutt-v3 } \\
\text { alizadeh-v3 } \\
\text { lapointe-v2 }\end{array}$ & $\begin{array}{l}\text { alizadeh-v1 } \\
\text { alizadeh-v2 } \\
\text { armstrong-v1 } \\
\text { bhattacharjee } \\
\text { bittner } \\
\text { chen } \\
\text { chowdary } \\
\text { golub-v1 } \\
\text { lapointe-v1 } \\
\text { liang } \\
\text { nutt-v1 } \\
\text { nutt-v2 } \\
\text { shipp-v1 } \\
\text { pomeroy-v1 } \\
\text { risinger } \\
\text { singh } \\
\text { tomlins-v1 } \\
\text { west }\end{array}$ & $\begin{array}{r}\text { nutt-v3 } \\
\text { alizadeh-v3 } \\
\text { lapointe-v2 } \\
\text { armstrong-v1 } \\
\text { tomlins-v1 }\end{array}$ & $\begin{array}{l}\text { alizadeh-v1 } \\
\text { alizadeh-v2 } \\
\text { bhattacharjee } \\
\text { bittner } \\
\text { chen } \\
\text { chowdary } \\
\text { golub-v1 } \\
\text { lapointe-v1 } \\
\text { liang } \\
\text { nutt-v1 } \\
\text { nutt-v2 } \\
\text { shipp-v1 } \\
\text { pomeroy-v1 } \\
\text { risinger } \\
\text { singh } \\
\text { west }\end{array}$ & $\begin{array}{r}\text { nutt-v3 } \\
\text { alizadeh-v3 } \\
\text { lapointe-v2 } \\
\text { armstrong-v1 } \\
\text { tomlins-v1 } \\
\text { bittner } \\
\text { risinger }\end{array}$ & $\begin{array}{l}\text { alizadeh-v1 } \\
\text { alizadeh-v2 } \\
\text { bhattacharjee } \\
\text { chen } \\
\text { chowdary } \\
\text { golub-v1 } \\
\text { lapointe-v1 } \\
\text { liang } \\
\text { nutt-v1 } \\
\text { nutt-v2 } \\
\text { shipp-v1 } \\
\text { pomeroy-v1 } \\
\text { singh } \\
\text { west }\end{array}$ & $\begin{array}{r}\text { nutt-v3 } \\
\text { alizadeh-v3 } \\
\text { lapointe-v2 } \\
\text { armstrong-v1 } \\
\text { tomlins-v1 } \\
\text { bittner } \\
\text { risinger } \\
\text { chowdary } \\
\text { west }\end{array}$ & $\begin{array}{l}\text { alizadeh-v1 } \\
\text { alizadeh-v2 } \\
\text { bhattacharjee } \\
\text { chen } \\
\text { golub-v1 } \\
\text { lapointe-v1 } \\
\text { liang } \\
\text { nutt-v1 } \\
\text { nutt-v2 } \\
\text { shipp-v1 } \\
\text { pomeroy-v1 } \\
\text { singh }\end{array}$ \\
\hline
\end{tabular}

\footnotetext{
${ }^{2}$ The values of $l$ and $c$ for each data set can be found at http://algorithmics.molgen.mpg.de/ Static/Supplements/CompCancer/datasets.htm
} 


\section{Parameter Optimisation}

Table 6.7 presents the results of the tuning experiments. We present the average ranking of each version of HEAD-DT $(\mathrm{H}-p)$ in the corresponding experimental configuration. For example, when $\mathrm{H}-0.1$ makes use of a single data set for evolving the optimal algorithm $(\{1 \times 13\})$, its performance in the remaining 13 data sets gives $\mathrm{H}-0.1$ the average rank position of 5.73 regarding the accuracy of its corresponding decision trees, and 6.15 regarding F-Measure.

Table 6.7: Results of the tuning experiments for the homogeneous. HEAD-DT is executed with different values of parameter $p(\mathrm{H}-p)$. Values are the average performance (rank) of each HEAD-DT version in the corresponding meta-test set of tuning data sets, according to either accuracy or F-Measure.

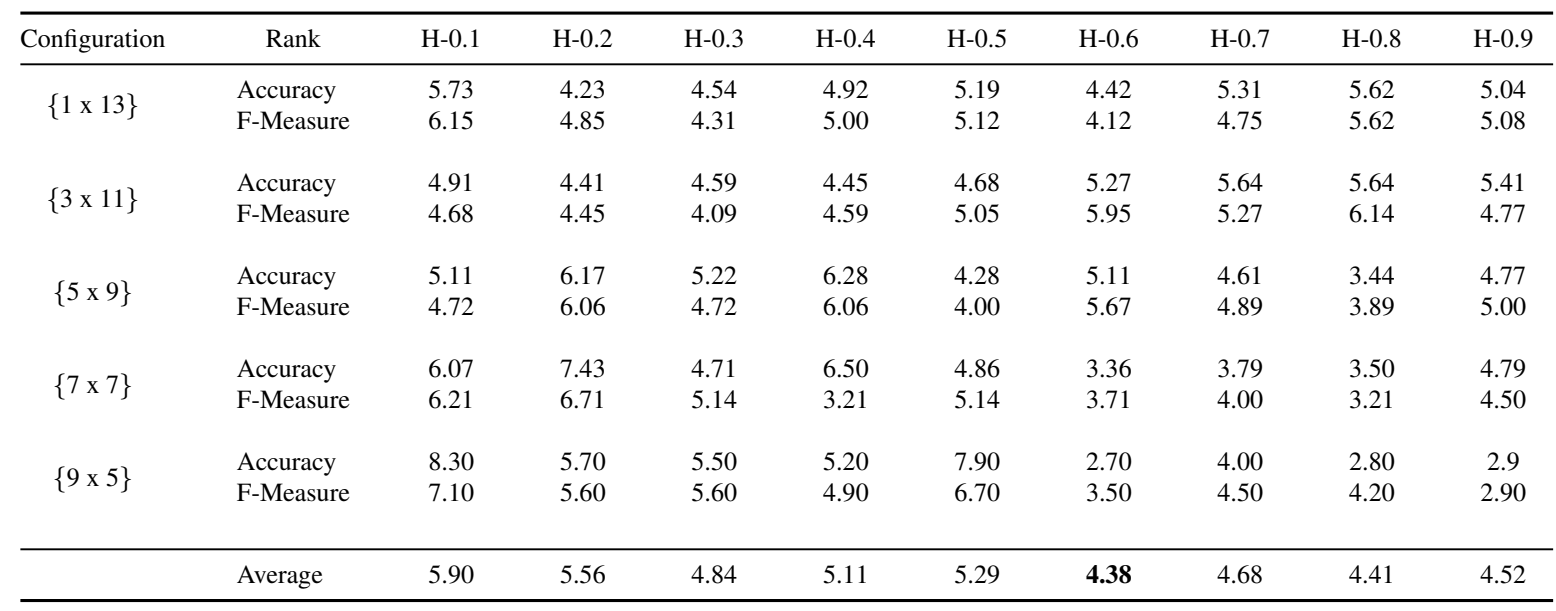

The Friedman and Nemenyi tests did not indicate any statistically significant differences among the 9 distinct versions of HEAD-DT, either considering accuracy or F-Measure, for any of the experimental configurations. This lack of significant differences indicates that HEAD-DT is robust to different values of $p$. For selecting the ideal value of $p$ to employ in the experimental analysis, we averaged the results across the different configurations, and also between the two different evaluation measures, accuracy and F-Measure. Hence, we calculated the average of the average ranks for each H- $p$ version across the distinct configurations and evaluation measures, and the results are presented in the bottom of Table 6.7. It is possible to see how marginal are the differences among values of $p \geq 0.6$. H- 0.6 was then selected as the optimised version of HEAD-DT, bearing in mind it presented the lowest average of the average ranks (4.38).

In the next section, we present the results for the experimental analysis performed over the 21 data sets in the experiments group, in which we compare H-0.6 (hereafter called simply HEAD-DT) to the baseline algorithms.

\section{Experimental Results}

Tables 6.8-6.12 show the average values of accuracy and F-Measure achieved by HEAD-DT, CART, C4.5, and REPTree in configurations $\{1 \times 20\},\{3 \times 18\},\{5 \times 16\},\{7 \times 14\}$, and $\{9 \times 12\}$. 
At the bottom of each table, we present the average rank position (the average of the rank position in each data set) of each method. The lower the ranking, the better the method. A method capable of outperforming any other method in every data set would have an average rank position of 1.00 (first place).

The first experiment was performed for configuration $\{1 \times 20\}$. Table 6.8 shows the results for this configuration considering accuracy (Table 6.8-(a)) and F-Measure (Table 6.8-(b)). Note that HEAD-DT is the best performing method with respect to both accuracy and F-Measure, reaching an average rank of 1.75 and 1.55 , respectively. HEAD-DT is followed by CART (2.45 and 2.50) and C4.5 (2.70 and 2.65), whose performances are quite evenly matched. REPTree is the worst-ranked method for both accuracy (3.10) and F-Measure (3.35).

Table 6.8: Results for the $\{1 \times 20\}$ configuration.

(a) Accuracy results.

\begin{tabular}{lrrrr}
\hline \multicolumn{1}{c}{ Data Set } & HEAD-DT & CART & C4.5 & REP \\
\hline alizadeh-2000-v1 & $0.77 \pm 0.11$ & $0.71 \pm 0.16$ & $0.68 \pm 0.20$ & $0.72 \pm 0.19$ \\
alizadeh-2000-v2 & $0.88 \pm 0.06$ & $0.92 \pm 0.11$ & $0.86 \pm 0.15$ & $0.84 \pm 0.17$ \\
alizadeh-2000-v3 & $0.71 \pm 0.05$ & $0.69 \pm 0.15$ & $0.71 \pm 0.15$ & $0.66 \pm 0.13$ \\
armstrong-2002-v1 & $0.90 \pm 0.04$ & $0.90 \pm 0.07$ & $0.89 \pm 0.06$ & $0.91 \pm 0.07$ \\
bhattacharjee-2001 & $0.90 \pm 0.03$ & $0.89 \pm 0.10$ & $0.89 \pm 0.08$ & $0.86 \pm 0.06$ \\
bittner-2000 & $0.61 \pm 0.09$ & $0.53 \pm 0.18$ & $0.49 \pm 0.16$ & $0.71 \pm 0.22$ \\
chen-2002 & $0.85 \pm 0.04$ & $0.85 \pm 0.07$ & $0.81 \pm 0.07$ & $0.79 \pm 0.12$ \\
chowdary-2006 & $0.95 \pm 0.03$ & $0.97 \pm 0.05$ & $0.95 \pm 0.05$ & $0.94 \pm 0.06$ \\
golub-1999-v1 & $0.88 \pm 0.03$ & $0.86 \pm 0.07$ & $0.88 \pm 0.08$ & $0.90 \pm 0.10$ \\
lapointe-2004-v1 & $0.66 \pm 0.08$ & $0.78 \pm 0.18$ & $0.71 \pm 0.14$ & $0.63 \pm 0.09$ \\
lapointe-2004-v2 & $0.62 \pm 0.05$ & $0.68 \pm 0.19$ & $0.57 \pm 0.11$ & $0.61 \pm 0.15$ \\
liang-2005 & $0.89 \pm 0.09$ & $0.71 \pm 0.14$ & $0.76 \pm 0.19$ & $0.78 \pm 0.18$ \\
nutt-2003-v1 & $0.53 \pm 0.08$ & $0.54 \pm 0.19$ & $0.50 \pm 0.17$ & $0.40 \pm 0.21$ \\
nutt-2003-v2 & $0.84 \pm 0.08$ & $0.77 \pm 0.21$ & $0.87 \pm 0.17$ & $0.45 \pm 0.27$ \\
pomeroy-2002-v1 & $0.88 \pm 0.08$ & $0.84 \pm 0.17$ & $0.88 \pm 0.16$ & $0.73 \pm 0.10$ \\
risinger-2003 & $0.58 \pm 0.15$ & $0.52 \pm 0.15$ & $0.53 \pm 0.19$ & $0.59 \pm 0.21$ \\
shipp-2002-v1 & $0.91 \pm 0.05$ & $0.77 \pm 0.12$ & $0.84 \pm 0.16$ & $0.77 \pm 0.05$ \\
singh-2002 & $0.78 \pm 0.04$ & $0.74 \pm 0.14$ & $0.78 \pm 0.12$ & $0.69 \pm 0.15$ \\
tomlins-2006 & $0.59 \pm 0.07$ & $0.58 \pm 0.20$ & $0.58 \pm 0.18$ & $0.58 \pm 0.10$ \\
west-2001 & $0.89 \pm 0.08$ & $0.89 \pm 0.11$ & $0.84 \pm 0.13$ & $0.74 \pm 0.19$ \\
\hline Average Rank & $\mathbf{1 . 7 5}$ & 2.45 & 2.70 & 3.10 \\
\hline
\end{tabular}

(b) F-Measure results.

\begin{tabular}{lrrrr}
\hline \multicolumn{1}{c}{ Data Set } & HEAD-DT & CART & C4.5 & REP \\
\hline alizadeh-2000-v1 & $0.77 \pm 0.11$ & $0.67 \pm 0.21$ & $0.63 \pm 0.26$ & $0.72 \pm 0.19$ \\
alizadeh-2000-v2 & $0.89 \pm 0.06$ & $0.92 \pm 0.10$ & $0.84 \pm 0.17$ & $0.80 \pm 0.19$ \\
alizadeh-2000-v3 & $0.71 \pm 0.05$ & $0.65 \pm 0.18$ & $0.68 \pm 0.17$ & $0.63 \pm 0.12$ \\
armstrong-2002-v1 & $0.90 \pm 0.04$ & $0.90 \pm 0.07$ & $0.88 \pm 0.06$ & $0.91 \pm 0.08$ \\
bhattacharjee-2001 & $0.89 \pm 0.02$ & $0.87 \pm 0.12$ & $0.89 \pm 0.08$ & $0.85 \pm 0.07$ \\
bittner-2000 & $0.61 \pm 0.09$ & $0.49 \pm 0.21$ & $0.45 \pm 0.19$ & $0.66 \pm 0.28$ \\
chen-2002 & $0.85 \pm 0.04$ & $0.85 \pm 0.07$ & $0.81 \pm 0.07$ & $0.78 \pm 0.13$ \\
chowdary-2006 & $0.95 \pm 0.03$ & $0.97 \pm 0.05$ & $0.95 \pm 0.05$ & $0.94 \pm 0.07$ \\
golub-1999-v1 & $0.88 \pm 0.03$ & $0.86 \pm 0.07$ & $0.87 \pm 0.08$ & $0.90 \pm 0.10$ \\
lapointe-2004-v1 & $0.64 \pm 0.10$ & $0.73 \pm 0.20$ & $0.68 \pm 0.15$ & $0.50 \pm 0.13$ \\
lapointe-2004-v2 & $0.62 \pm 0.05$ & $0.66 \pm 0.20$ & $0.58 \pm 0.10$ & $0.57 \pm 0.15$ \\
liang-2005 & $0.87 \pm 0.10$ & $0.67 \pm 0.21$ & $0.77 \pm 0.22$ & $0.71 \pm 0.23$ \\
nutt-2003-v1 & $0.53 \pm 0.08$ & $0.48 \pm 0.19$ & $0.46 \pm 0.16$ & $0.31 \pm 0.22$ \\
nutt-2003-v2 & $0.84 \pm 0.08$ & $0.73 \pm 0.26$ & $0.87 \pm 0.17$ & $0.34 \pm 0.31$ \\
pomeroy-2002-v1 & $0.87 \pm 0.10$ & $0.79 \pm 0.22$ & $0.85 \pm 0.20$ & $0.62 \pm 0.14$ \\
risinger-2003 & $0.56 \pm 0.15$ & $0.48 \pm 0.19$ & $0.53 \pm 0.21$ & $0.52 \pm 0.25$ \\
shipp-2002-v1 & $0.90 \pm 0.05$ & $0.75 \pm 0.13$ & $0.83 \pm 0.17$ & $0.70 \pm 0.10$ \\
singh-2002 & $0.78 \pm 0.04$ & $0.72 \pm 0.18$ & $0.77 \pm 0.13$ & $0.67 \pm 0.18$ \\
tomlins-2006 & $0.58 \pm 0.08$ & $0.56 \pm 0.20$ & $0.56 \pm 0.20$ & $0.53 \pm 0.11$ \\
west-2001 & $0.89 \pm 0.08$ & $0.89 \pm 0.12$ & $0.81 \pm 0.17$ & $0.71 \pm 0.23$ \\
\hline Average Rank & $\mathbf{1 . 5 5}$ & 2.50 & 2.60 & 3.35 \\
\hline
\end{tabular}

The next experiment concerns configuration $\{3 \times 18\}$, whose results are presented in Table 6.9. In this experiment, HEAD-DT's average rank is again the lowest of the experiment: 1.61 for both accuracy and F-Measure. That means HEAD-DT is often the best performing method (1st place) in the group of 18 test data sets. CART and $\mathrm{C} 4.5$ once again present very similar average rank values, which is not surprising bearing in mind they are both considered the state-of-the-art top-down decision-tree induction algorithms. REPTree is again the worst-performing method among the four algorithms.

Table 6.10 presents the results for configuration $\left\{\begin{array}{lll}5 \times 16 & 1\end{array}\right.$. The scenario is quite similar to the previous configurations, with HEAD-DT leading the ranking, with average rank values of 1.44 (accuracy) and 1.31 (F-Measure). These are the lowest rank values obtained by a method in any experimental configuration conducted in this analysis. HEAD-DT is followed by CART (2.69 and 2.69) and C4.5 (2.69 and 2.63), which once again present very similar performances. REPTree is at the bottom of the ranking, with 3.19 (accuracy) and 3.38 (F-Measure).

The experimental results for configuration $\{7 \times 14\}$ show a different picture. Table 6.11-(a), which depicts the accuracy values of each method, indicates that HEAD-DT is outperformed by 
Table 6.9: Results for the $\{3 \times 18\}$ configuration.

(a) Accuracy results.

\begin{tabular}{|c|c|c|c|c|}
\hline Data Set & HEAD-DT & CART & C4.5 & REP \\
\hline alizadeh-2000-v1 & $0.74 \pm 0.07$ & $0.71 \pm 0.16$ & $0.68 \pm 0.20$ & $0.72 \pm 0.19$ \\
\hline alizadeh-2000-v2 & $0.90 \pm 0.07$ & $0.92 \pm 0.11$ & $0.86 \pm 0.15$ & $0.84 \pm 0.17$ \\
\hline armstrong-2002-v1 & $0.88 \pm 0.01$ & $0.90 \pm 0.07$ & $0.89 \pm 0.06$ & $0.91 \pm 0.07$ \\
\hline bhattacharjee-2001 & $0.92 \pm 0.03$ & $0.89 \pm 0.10$ & $0.89 \pm 0.08$ & $0.86 \pm 0.06$ \\
\hline bittner-2000 & $0.55 \pm 0.09$ & $0.53 \pm 0.18$ & $0.49 \pm 0.16$ & $0.71 \pm 0.22$ \\
\hline chen-2002 & $0.87 \pm 0.03$ & $0.85 \pm 0.07$ & $0.81 \pm 0.07$ & $0.79 \pm 0.12$ \\
\hline chowdary-2006 & $0.97 \pm 0.01$ & $0.97 \pm 0.05$ & $0.95 \pm 0.05$ & $0.94 \pm 0.06$ \\
\hline golub-1999-v1 & $0.89 \pm 0.02$ & $0.86 \pm 0.07$ & $0.88 \pm 0.08$ & $0.90 \pm 0.10$ \\
\hline lapointe-2004-v1 & $0.70 \pm 0.06$ & $0.78 \pm 0.18$ & $0.71 \pm 0.14$ & $0.63 \pm 0.09$ \\
\hline liang-2005 & $0.89 \pm 0.08$ & $0.71 \pm 0.14$ & $0.76 \pm 0.19$ & $0.78 \pm 0.18$ \\
\hline nutt-2003-v1 & $0.67 \pm 0.10$ & $0.54 \pm 0.19$ & $0.50 \pm 0.17$ & $0.40 \pm 0.21$ \\
\hline nutt-2003-v2 & $0.77 \pm 0.08$ & $0.77 \pm 0.21$ & $0.87 \pm 0.17$ & $0.45 \pm 0.27$ \\
\hline pomeroy-2002-v1 & $0.92 \pm 0.05$ & $0.84 \pm 0.17$ & $0.88 \pm 0.16$ & $0.73 \pm 0.10$ \\
\hline risinger-2003 & $0.53 \pm 0.11$ & $0.52 \pm 0.15$ & $0.53 \pm 0.19$ & $0.59 \pm 0.21$ \\
\hline shipp-2002-v1 & $0.85 \pm 0.05$ & $0.77 \pm 0.12$ & $0.84 \pm 0.16$ & $0.77 \pm 0.05$ \\
\hline singh-2002 & $0.80 \pm 0.04$ & $0.74 \pm 0.14$ & $0.78 \pm 0.12$ & $0.69 \pm 0.15$ \\
\hline tomlins-2006 & $0.64 \pm 0.03$ & $0.58 \pm 0.20$ & $0.58 \pm 0.18$ & $0.58 \pm 0.10$ \\
\hline west-2001 & $0.92 \pm 0.04$ & $0.90 \pm 0.11$ & $0.84 \pm 0.13$ & $0.74 \pm 0.19$ \\
\hline Average Rank & 1.61 & 2.61 & 2.72 & 3.05 \\
\hline
\end{tabular}

(b) F-Measure results.

\begin{tabular}{lrrrr}
\hline \multicolumn{1}{c}{ Data Set } & HEAD-DT & CART & C4.5 & REP \\
\hline alizadeh-2000-v1 & $0.73 \pm 0.07$ & $0.67 \pm 0.21$ & $0.63 \pm 0.26$ & $0.72 \pm 0.19$ \\
alizadeh-2000-v2 & $0.91 \pm 0.06$ & $0.92 \pm 0.10$ & $0.84 \pm 0.17$ & $0.80 \pm 0.19$ \\
armstrong-2002-v1 & $0.88 \pm 0.02$ & $0.90 \pm 0.07$ & $0.88 \pm 0.06$ & $0.91 \pm 0.08$ \\
bhattacharee-2001 & $0.91 \pm 0.03$ & $0.87 \pm 0.12$ & $0.89 \pm 0.08$ & $0.85 \pm 0.07$ \\
bittner-2000 & $0.53 \pm 0.10$ & $0.49 \pm 0.21$ & $0.45 \pm 0.19$ & $0.66 \pm 0.28$ \\
chen-2002 & $0.87 \pm 0.03$ & $0.85 \pm 0.07$ & $0.81 \pm 0.07$ & $0.78 \pm 0.13$ \\
chowdary-2006 & $0.97 \pm 0.01$ & $0.97 \pm 0.05$ & $0.95 \pm 0.05$ & $0.94 \pm 0.07$ \\
golub-1999-v1 & $0.89 \pm 0.02$ & $0.86 \pm 0.07$ & $0.87 \pm 0.08$ & $0.90 \pm 0.10$ \\
lapointe-2004-v1 & $0.68 \pm 0.08$ & $0.73 \pm 0.20$ & $0.68 \pm 0.15$ & $0.50 \pm 0.13$ \\
liang-2005 & $0.87 \pm 0.10$ & $0.67 \pm 0.21$ & $0.77 \pm 0.22$ & $0.71 \pm 0.23$ \\
nutt-2003-v1 & $0.65 \pm 0.10$ & $0.48 \pm 0.19$ & $0.46 \pm 0.16$ & $0.31 \pm 0.22$ \\
nutt-2003-v2 & $0.77 \pm 0.08$ & $0.73 \pm 0.26$ & $0.87 \pm 0.17$ & $0.34 \pm 0.31$ \\
pomeroy-2002-v1 & $0.92 \pm 0.05$ & $0.79 \pm 0.22$ & $0.85 \pm 0.20$ & $0.62 \pm 0.14$ \\
risinger-2003 & $0.52 \pm 0.11$ & $0.48 \pm 0.19$ & $0.53 \pm 0.21$ & $0.52 \pm 0.25$ \\
shipp-2002-v1 & $0.84 \pm 0.05$ & $0.75 \pm 0.13$ & $0.83 \pm 0.17$ & $0.70 \pm 0.10$ \\
singh-2002 & $0.80 \pm 0.04$ & $0.72 \pm 0.18$ & $0.77 \pm 0.13$ & $0.67 \pm 0.18$ \\
tomlins-2006 & $0.63 \pm 0.03$ & $0.56 \pm 0.20$ & $0.56 \pm 0.20$ & $0.53 \pm 0.11$ \\
west-2001 & $0.92 \pm 0.04$ & $0.89 \pm 0.12$ & $0.81 \pm 0.17$ & $0.71 \pm 0.23$ \\
\hline Average Rank & & & & \\
\hline
\end{tabular}

Table 6.10: Results for the $\{5 \times 16\}$ configuration.

(a) Accuracy results.

\begin{tabular}{lrrrr}
\hline \multicolumn{1}{c}{ Data Set } & HEAD-DT & CART & C4.5 & REP \\
\hline alizadeh-2000-v1 & $0.75 \pm 0.05$ & $0.71 \pm 0.16$ & $0.68 \pm 0.20$ & $0.72 \pm 0.19$ \\
alizadeh-2000-v2 & $0.91 \pm 0.07$ & $0.92 \pm 0.11$ & $0.86 \pm 0.15$ & $0.84 \pm 0.17$ \\
bhattacharjee-2001 & $0.94 \pm 0.02$ & $0.89 \pm 0.10$ & $0.89 \pm 0.08$ & $0.86 \pm 0.06$ \\
bittner-2000 & $0.54 \pm 0.08$ & $0.53 \pm 0.18$ & $0.49 \pm 0.16$ & $0.71 \pm 0.22$ \\
chen-2002 & $0.91 \pm 0.02$ & $0.85 \pm 0.07$ & $0.81 \pm 0.07$ & $0.79 \pm 0.12$ \\
chowdary-2006 & $0.97 \pm 0.01$ & $0.97 \pm 0.05$ & $0.95 \pm 0.05$ & $0.94 \pm 0.06$ \\
golub-1999-v1 & $0.88 \pm 0.02$ & $0.86 \pm 0.07$ & $0.88 \pm 0.08$ & $0.90 \pm 0.10$ \\
lapointe-2004-v1 & $0.71 \pm 0.06$ & $0.78 \pm 0.18$ & $0.71 \pm 0.14$ & $0.63 \pm 0.09$ \\
liang-2005 & $0.89 \pm 0.08$ & $0.71 \pm 0.14$ & $0.76 \pm 0.19$ & $0.78 \pm 0.18$ \\
nutt-2003-v1 & $0.61 \pm 0.10$ & $0.54 \pm 0.19$ & $0.50 \pm 0.17$ & $0.40 \pm 0.21$ \\
nutt-2003-v2 & $0.78 \pm 0.08$ & $0.77 \pm 0.21$ & $0.87 \pm 0.17$ & $0.45 \pm 0.27$ \\
pomeroy-2002-v1 & $0.92 \pm 0.05$ & $0.84 \pm 0.17$ & $0.88 \pm 0.16$ & $0.73 \pm 0.10$ \\
risinger-2003 & $0.55 \pm 0.08$ & $0.52 \pm 0.15$ & $0.53 \pm 0.19$ & $0.59 \pm 0.21$ \\
shipp-2002-v1 & $0.87 \pm 0.03$ & $0.77 \pm 0.12$ & $0.84 \pm 0.16$ & $0.77 \pm 0.05$ \\
singh-2002 & $0.78 \pm 0.03$ & $0.74 \pm 0.14$ & $0.78 \pm 0.12$ & $0.69 \pm 0.15$ \\
west-2001 & $0.92 \pm 0.03$ & $0.90 \pm 0.11$ & $0.84 \pm 0.13$ & $0.74 \pm 0.19$ \\
\hline Average Rank & $\mathbf{1 . 4 4}$ & 2.69 & 2.69 & 3.19 \\
\hline
\end{tabular}

(b) F-Measure results.

\begin{tabular}{lrrrr}
\hline \multicolumn{1}{c}{ Data Set } & HEAD-DT & CART & C4.5 & REP \\
\hline alizadeh-2000-v1 & $0.75 \pm 0.05$ & $0.67 \pm 0.21$ & $0.63 \pm 0.26$ & $0.72 \pm 0.19$ \\
alizadeh-2000-v2 & $0.91 \pm 0.06$ & $0.92 \pm 0.10$ & $0.84 \pm 0.17$ & $0.80 \pm 0.19$ \\
bhattacharjee-2001 & $0.94 \pm 0.02$ & $0.87 \pm 0.12$ & $0.89 \pm 0.08$ & $0.85 \pm 0.07$ \\
bittner-2000 & $0.53 \pm 0.09$ & $0.49 \pm 0.21$ & $0.45 \pm 0.19$ & $0.66 \pm 0.28$ \\
chen-2002 & $0.91 \pm 0.02$ & $0.85 \pm 0.07$ & $0.81 \pm 0.07$ & $0.78 \pm 0.13$ \\
chowdary-2006 & $0.97 \pm 0.01$ & $0.97 \pm 0.05$ & $0.95 \pm 0.05$ & $0.94 \pm 0.07$ \\
golub-1999-v1 & $0.88 \pm 0.02$ & $0.86 \pm 0.07$ & $0.87 \pm 0.08$ & $0.90 \pm 0.10$ \\
lapointe-2004-v1 & $0.69 \pm 0.08$ & $0.73 \pm 0.20$ & $0.68 \pm 0.15$ & $0.50 \pm 0.13$ \\
liang-2005 & $0.87 \pm 0.10$ & $0.67 \pm 0.21$ & $0.77 \pm 0.22$ & $0.71 \pm 0.23$ \\
nutt-2003-v1 & $0.60 \pm 0.09$ & $0.48 \pm 0.19$ & $0.46 \pm 0.16$ & $0.31 \pm 0.22$ \\
nutt-2003-v2 & $0.78 \pm 0.08$ & $0.73 \pm 0.26$ & $0.87 \pm 0.17$ & $0.34 \pm 0.31$ \\
pomeroy-2002-v1 & $0.92 \pm 0.05$ & $0.79 \pm 0.22$ & $0.85 \pm 0.20$ & $0.62 \pm 0.14$ \\
risinger-2003 & $0.53 \pm 0.08$ & $0.48 \pm 0.19$ & $0.53 \pm 0.21$ & $0.52 \pm 0.25$ \\
shipp-2002-v1 & $0.86 \pm 0.03$ & $0.75 \pm 0.13$ & $0.83 \pm 0.17$ & $0.70 \pm 0.10$ \\
singh-2002 & $0.78 \pm 0.03$ & $0.72 \pm 0.18$ & $0.77 \pm 0.13$ & $0.67 \pm 0.18$ \\
west-2001 & $0.92 \pm 0.03$ & $0.89 \pm 0.12$ & $0.81 \pm 0.17$ & $0.71 \pm 0.23$ \\
\hline Average Rank & $\mathbf{1 . 3 1}$ & 2.69 & 2.63 & 3.38 \\
\hline
\end{tabular}


both CART and C4.5, though their average rank values are very similar: $2.14,2.21$, versus 2.36 for HEAD-DT. REPTree keeps its position as worst-performing method, with an average rank value of 3.29. However, Table 6.11-(b) returns to the same scenario presented in configurations $\{1 \times 20\},\{3 \times 18\}$, and $\{5 \times 16\}$ : HEAD-DT outperforming the baseline methods, with CART and C4.5 tied in the second place and REPTree in the last position. The effect seen in Table 6.11-(a), in which HEAD-DT did not outperform the baseline methods, has a straightforward explanation: HEAD-DT optimises its generated algorithms according to the F-Measure evaluation measure. Since accuracy may be a misleading measure (it is not suitable for data sets with imbalanced class distributions), and several of the gene-expression data sets are imbalanced (i.e., they have a large difference in the relative frequency of the most frequent and the least frequent classes in the data set), it seems fair to say that HEAD-DT also outperformed the baseline methods in configuration $\{7 \times 14\}$, given that it generated algorithms whose F-Measure values are better than the values achieved by CART, C4.5, and REPTree.

Table 6.11: Results for the $\{7 \times 14\}$ configuration.

(a) Accuracy results.

\begin{tabular}{lrrrr}
\hline \multicolumn{1}{c}{ Data Set } & HEAD-DT & CART & C4.5 & REP \\
\hline alizadeh-2000-v1 & $0.79 \pm 0.09$ & $0.71 \pm 0.16$ & $0.68 \pm 0.20$ & $0.72 \pm 0.19$ \\
alizadeh-2000-v2 & $0.89 \pm 0.05$ & $0.92 \pm 0.11$ & $0.86 \pm 0.15$ & $0.84 \pm 0.17$ \\
bhattacharjee-2001 & $0.89 \pm 0.03$ & $0.89 \pm 0.10$ & $0.89 \pm 0.08$ & $0.86 \pm 0.06$ \\
chen-2002 & $0.84 \pm 0.02$ & $0.85 \pm 0.07$ & $0.81 \pm 0.07$ & $0.79 \pm 0.12$ \\
chowdary-2006 & $0.91 \pm 0.04$ & $0.97 \pm 0.05$ & $0.95 \pm 0.05$ & $0.94 \pm 0.06$ \\
golub-1999-v1 & $0.84 \pm 0.03$ & $0.86 \pm 0.07$ & $0.88 \pm 0.08$ & $0.90 \pm 0.10$ \\
lapointe-2004-v1 & $0.61 \pm 0.09$ & $0.78 \pm 0.18$ & $0.71 \pm 0.14$ & $0.63 \pm 0.09$ \\
liang-2005 & $0.87 \pm 0.08$ & $0.71 \pm 0.14$ & $0.76 \pm 0.19$ & $0.78 \pm 0.18$ \\
nutt-2003-v1 & $0.68 \pm 0.07$ & $0.54 \pm 0.19$ & $0.50 \pm 0.17$ & $0.40 \pm 0.21$ \\
nutt-2003-v2 & $0.76 \pm 0.07$ & $0.77 \pm 0.21$ & $0.87 \pm 0.17$ & $0.45 \pm 0.27$ \\
pomeroy-2002-v1 & $0.70 \pm 0.12$ & $0.84 \pm 0.17$ & $0.88 \pm 0.16$ & $0.73 \pm 0.10$ \\
shipp-2002-v1 & $0.86 \pm 0.03$ & $0.77 \pm 0.12$ & $0.84 \pm 0.16$ & $0.77 \pm 0.05$ \\
singh-2002 & $0.79 \pm 0.02$ & $0.74 \pm 0.14$ & $0.78 \pm 0.12$ & $0.69 \pm 0.15$ \\
west-2001 & $0.80 \pm 0.07$ & $0.90 \pm 0.11$ & $0.84 \pm 0.13$ & $0.74 \pm 0.19$ \\
\hline Average Rank & 2.36 & $\mathbf{2 . 1 4}$ & 2.21 & 3.29 \\
\hline
\end{tabular}

(b) F-Measure results.

\begin{tabular}{lrrrr}
\hline \multicolumn{1}{c}{ Data Set } & HEAD-DT & CART & C4.5 & REP \\
\hline alizadeh-2000-v1 & $0.79 \pm 0.09$ & $0.67 \pm 0.21$ & $0.63 \pm 0.26$ & $0.72 \pm 0.19$ \\
alizadeh-2000-v2 & $0.91 \pm 0.03$ & $0.92 \pm 0.10$ & $0.84 \pm 0.17$ & $0.80 \pm 0.19$ \\
bhattacharjee-2001 & $0.89 \pm 0.03$ & $0.87 \pm 0.12$ & $0.89 \pm 0.08$ & $0.85 \pm 0.07$ \\
chen-2002 & $0.84 \pm 0.02$ & $0.85 \pm 0.07$ & $0.81 \pm 0.07$ & $0.78 \pm 0.13$ \\
chowdary-2006 & $0.91 \pm 0.04$ & $0.97 \pm 0.05$ & $0.95 \pm 0.05$ & $0.94 \pm 0.07$ \\
golub-1999-v1 & $0.83 \pm 0.04$ & $0.86 \pm 0.07$ & $0.87 \pm 0.08$ & $0.90 \pm 0.10$ \\
lapointe-2004-v1 & $0.60 \pm 0.11$ & $0.73 \pm 0.20$ & $0.68 \pm 0.15$ & $0.50 \pm 0.13$ \\
liang-2005 & $0.87 \pm 0.08$ & $0.67 \pm 0.21$ & $0.77 \pm 0.22$ & $0.71 \pm 0.23$ \\
nutt-2003-v1 & $0.65 \pm 0.08$ & $0.48 \pm 0.19$ & $0.46 \pm 0.16$ & $0.31 \pm 0.22$ \\
nutt-2003-v2 & $0.76 \pm 0.07$ & $0.73 \pm 0.26$ & $0.87 \pm 0.17$ & $0.34 \pm 0.31$ \\
pomeroy-2002-v1 & $0.69 \pm 0.13$ & $0.79 \pm 0.22$ & $0.85 \pm 0.20$ & $0.62 \pm 0.14$ \\
shipp-2002-v1 & $0.86 \pm 0.03$ & $0.75 \pm 0.13$ & $0.83 \pm 0.17$ & $0.70 \pm 0.10$ \\
singh-2002 & $0.79 \pm 0.02$ & $0.72 \pm 0.18$ & $0.77 \pm 0.13$ & $0.67 \pm 0.18$ \\
west-2001 & $0.80 \pm 0.07$ & $0.89 \pm 0.12$ & $0.81 \pm 0.17$ & $0.71 \pm 0.23$ \\
\hline Average Rank & $\mathbf{2 . 0 7}$ & 2.21 & 2.21 & 3.50 \\
\hline
\end{tabular}

Table 6.12 shows the results for configuration $\{9 \times 12\}$. They are consistent to the previous configurations, in which HEAD-DT has the edge over the baseline methods, presenting the lowest average rank for both accuracy and F-Measure (1.58 and 1.42). C4.5 and CART presented similar ranks, whereas REPTree occupied the last position in the ranking.

Table 6.12: Results for the $\{9 \times 12\}$ configuration.

(a) Accuracy results.

\begin{tabular}{lrrrr}
\hline \multicolumn{1}{c}{ Data Set } & HEAD-DT & CART & C4.5 & REP \\
\hline alizadeh-2000-v1 & $0.79 \pm 0.09$ & $0.71 \pm 0.16$ & $0.68 \pm 0.20$ & $0.72 \pm 0.19$ \\
alizadeh-2000-v2 & $0.89 \pm 0.05$ & $0.92 \pm 0.11$ & $0.86 \pm 0.15$ & $0.84 \pm 0.17$ \\
bhattacharjee-2001 & $0.90 \pm 0.02$ & $0.89 \pm 0.10$ & $0.89 \pm 0.08$ & $0.86 \pm 0.06$ \\
chen-2002 & $0.88 \pm 0.03$ & $0.85 \pm 0.07$ & $0.81 \pm 0.07$ & $0.79 \pm 0.12$ \\
golub-1999-v1 & $0.87 \pm 0.02$ & $0.86 \pm 0.07$ & $0.88 \pm 0.08$ & $0.90 \pm 0.10$ \\
lapointe-2004-v1 & $0.70 \pm 0.05$ & $0.78 \pm 0.18$ & $0.71 \pm 0.14$ & $0.63 \pm 0.09$ \\
liang-2005 & $0.88 \pm 0.08$ & $0.71 \pm 0.14$ & $0.76 \pm 0.19$ & $0.78 \pm 0.18$ \\
nutt-2003-v1 & $0.67 \pm 0.09$ & $0.54 \pm 0.19$ & $0.50 \pm 0.17$ & $0.40 \pm 0.21$ \\
nutt-2003-v2 & $0.77 \pm 0.07$ & $0.77 \pm 0.21$ & $0.87 \pm 0.17$ & $0.45 \pm 0.27$ \\
shipp-2002-v1 & $0.86 \pm 0.03$ & $0.84 \pm 0.17$ & $0.88 \pm 0.16$ & $0.73 \pm 0.10$ \\
pomeroy-2002-v1 & $0.92 \pm 0.05$ & $0.77 \pm 0.12$ & $0.84 \pm 0.16$ & $0.77 \pm 0.05$ \\
singh-2002 & $0.82 \pm 0.04$ & $0.74 \pm 0.14$ & $0.78 \pm 0.12$ & $0.69 \pm 0.15$ \\
\hline \multicolumn{1}{c}{ Average Rank } & $\mathbf{1 . 5 8}$ & 2.67 & 2.33 & 3.42 \\
\hline
\end{tabular}

(b) F-Measure results.

\begin{tabular}{lrrrr}
\hline \multicolumn{1}{c}{ Data Set } & HEAD-DT & CART & C4.5 & REP \\
\hline alizadeh-2000-v1 & $0.78 \pm 0.09$ & $0.67 \pm 0.21$ & $0.63 \pm 0.26$ & $0.72 \pm 0.19$ \\
alizadeh-2000-v2 & $0.91 \pm 0.03$ & $0.92 \pm 0.10$ & $0.84 \pm 0.17$ & $0.80 \pm 0.19$ \\
bhattacharjee-2001 & $0.89 \pm 0.02$ & $0.87 \pm 0.12$ & $0.89 \pm 0.08$ & $0.85 \pm 0.07$ \\
chen-2002 & $0.88 \pm 0.03$ & $0.85 \pm 0.07$ & $0.81 \pm 0.07$ & $0.78 \pm 0.13$ \\
golub-1999-v1 & $0.87 \pm 0.02$ & $0.86 \pm 0.07$ & $0.87 \pm 0.08$ & $0.90 \pm 0.10$ \\
lapointe-2004-v1 & $0.68 \pm 0.07$ & $0.73 \pm 0.20$ & $0.68 \pm 0.15$ & $0.50 \pm 0.13$ \\
liang-2005 & $0.87 \pm 0.09$ & $0.67 \pm 0.21$ & $0.77 \pm 0.22$ & $0.71 \pm 0.23$ \\
nutt-2003-v1 & $0.66 \pm 0.09$ & $0.48 \pm 0.19$ & $0.46 \pm 0.16$ & $0.31 \pm 0.22$ \\
nutt-2003-v2 & $0.77 \pm 0.07$ & $0.73 \pm 0.26$ & $0.87 \pm 0.17$ & $0.34 \pm 0.31$ \\
shipp-2002-v1 & $0.86 \pm 0.03$ & $0.79 \pm 0.22$ & $0.85 \pm 0.20$ & $0.62 \pm 0.14$ \\
pomeroy-2002-v1 & $0.92 \pm 0.05$ & $0.75 \pm 0.13$ & $0.83 \pm 0.17$ & $0.70 \pm 0.10$ \\
singh-2002 & $0.82 \pm 0.04$ & $0.72 \pm 0.18$ & $0.77 \pm 0.13$ & $0.67 \pm 0.18$ \\
\hline Average Rank & $\mathbf{1 . 4 2}$ & 2.67 & 2.42 & 3.50 \\
\hline
\end{tabular}


Finally, Figure 6.1 presents the fitness evolution in HEAD-DT across a full evolutionary cycle of 100 generations. We present both mean and best fitness of the population in a given generation, for all experimental configurations. Some interesting observations can be extracted from Figure 6.1. First, when the meta-training set comprises a single data set (Figure 6.1-(a)), HEAD-DT is capable of continuously increase the fitness function value, and at the same time the population is reasonably heterogeneous (mean fitness value oscillates considerably). In the other extreme, when the meta-training set comprises 9 data sets (Figure 6.1-(e)), HEAD-DT has a harder time in optimising the fitness values, and the population is reasonably homogeneous (mean fitness value does not oscillate so much). The explanation for this behavior is that by increasing the number of data sets in the meta-training set, HEAD-DT has to find algorithms with a good performance trade-off within the meta-training set. In practice, modifications in the design of the algorithm that favor a given data set may very well harm another, and, hence, it is intuitively harder to design an algorithm that improves the performance of the generated decision trees in several data sets than in a single one. Conversely, it is also intuitive that a larger meta-training set leads to the design of a better "all-around" algorithm, i.e., an algorithm that is robust to the peculiarities of the data sets from the application domain.

\section{Discussion}

The experimental analysis conducted in the previous section aimed at comparing HEAD-DT to three baseline algorithms: CART, C4.5, and REPTree. We measured the performance of each algorithm according to accuracy and F-Measure, which are the most well-known criteria for evaluating classification algorithms. For verifying whether the number of data sets used in the meta-training set had an impact in the evolution of algorithms, we employed a consistent methodology that incrementally added random data sets from the set of available data, generating five different experimental configurations \{\#meta-training sets, \#meta-test sets $\}:\{1 \times 20\}$, $\{3 \times 18\},\{5 \times 16\},\{7 \times 14\}$, and $\{9 \times 12\}$. By analysing the average rank obtained by each method in the previously mentioned configurations, we conclude that:

- HEAD-DT is consistently the best-performing method, presenting the lowest average rank values among the four algorithms employed in the experimental analysis;

- C4.5 and CART's predictive performances are quite similar, which is consistent to the fact that both algorithms are still the state-of-the-art top-down decision-tree induction algorithms;

- REPTree, which is a variation of C4.5 that employs the reduced-error pruning strategy for simplifying the generated decision trees, is the worst-performing method in the group. Its disappointing results seem to indicate that the reduced-error pruning strategy is not particularly suited to the gene-expression data sets, probably because it requires an additional validation set. As previously observed, the gene-expression data sets have very few instances when compared to the currently giant databases from distinct application domains, and 


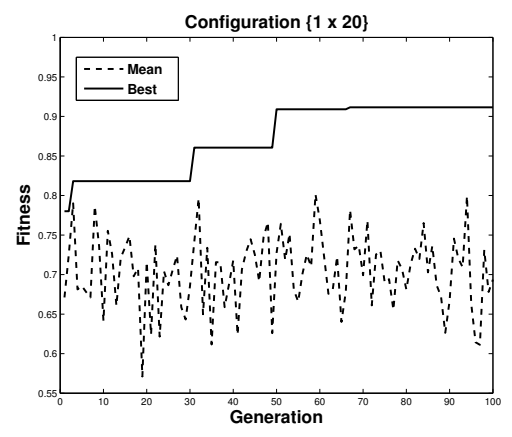

(a)

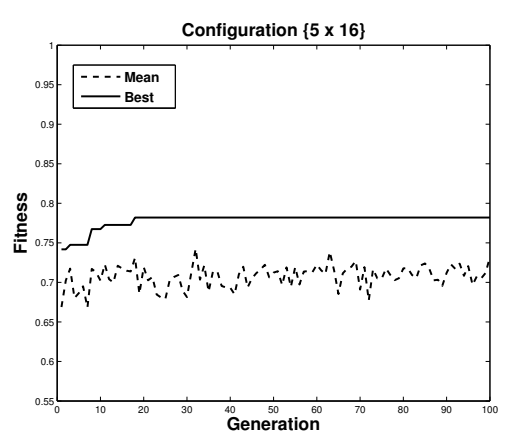

(c)

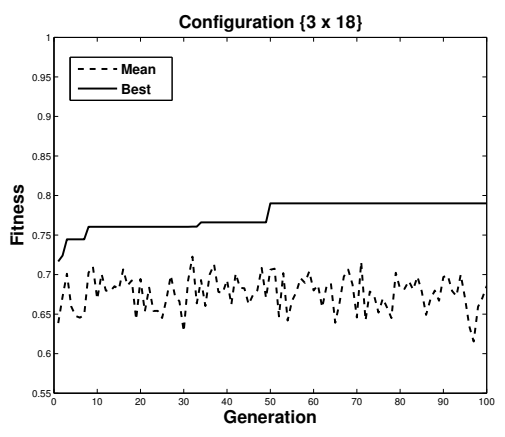

(b)

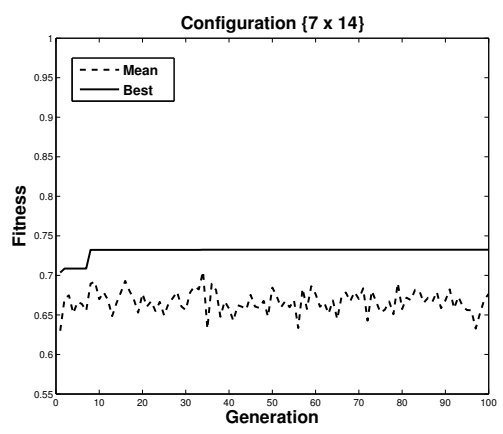

(d)

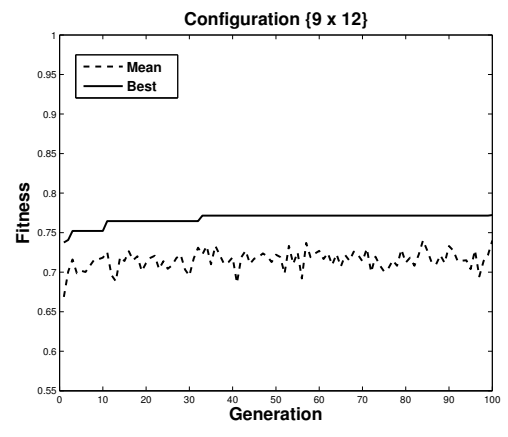

(e)

Figure 6.1: Fitness evolution in HEAD-DT. 
reducing their size for producing a validation set has certainly harmed REPTree's overall predictive performance.

For summarizing the average rank values obtained by each method in every experimental configuration, we gathered the rank values from Tables 6.8-6.12 in Table 6.13. Values in bold indicate the best performing method according to the corresponding evaluation measure. These results show that HEAD-DT consistently presents the lowest average rank.

Table 6.13: Summary of the experimental analysis regarding the homogeneous approach.

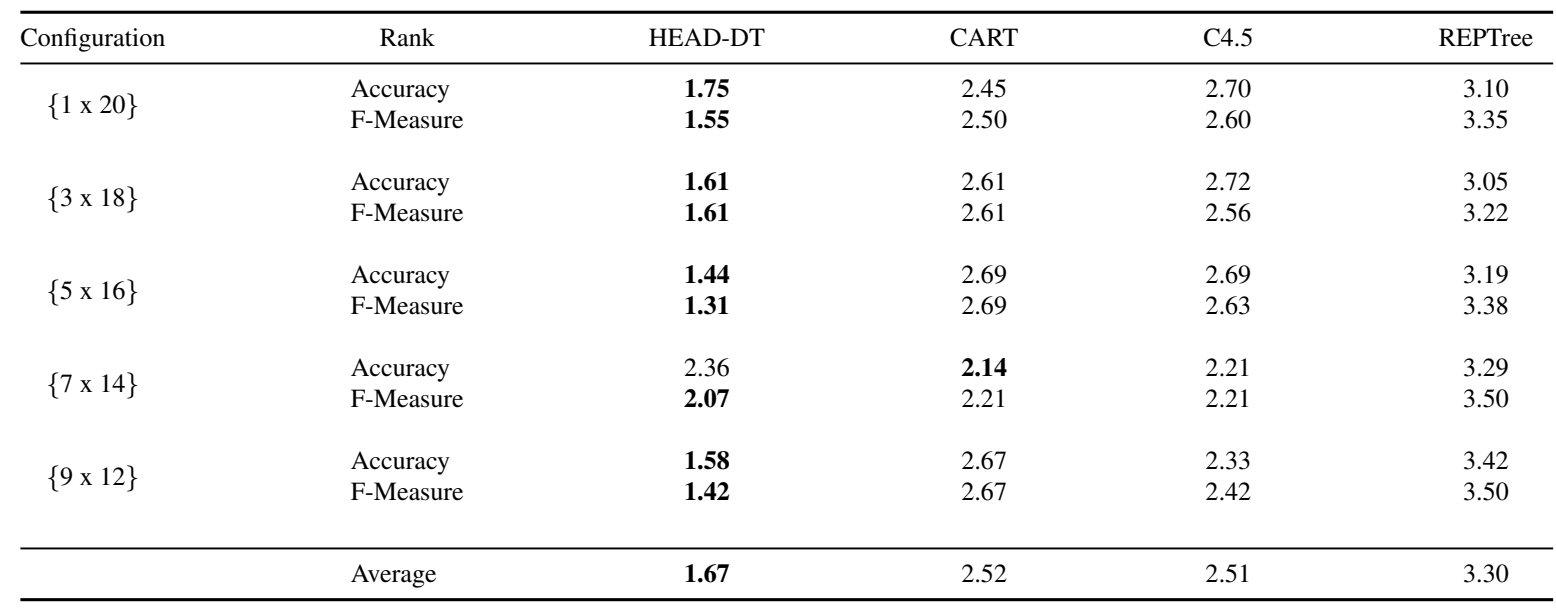

The last step of this empirical analysis is to verify whether the differences in rank values are statistically significant. For this particular analysis, we employ the graphical representation suggested by Demšar (2006), the so-called critical diagrams. In this diagram, a horizontal line represents the axis on which we plot the average rank values of the methods. The axis is turned so that the lowest (best) ranks are to the right, since we perceive the methods on the right side as better. When comparing all the algorithms against each other, we connect the groups of algorithms that are not significantly different through a bold horizontal line. We also show the critical difference given by the Nemenyi test in the top of the graph.

Figure 6.2 shows the critical diagrams for all experimental configurations. Note that HEAD-DT outperforms C4.5, CART, and REPTree with statistical significance in configuration $\left\{\begin{array}{lll}5 \times & 16\end{array}\right\}$ for both accuracy (Figure 6.2-(e)) and F-Measure (Figure 6.2-(f)). The only scenarios in which there were no statistically significant differences between all methods are related to the accuracy measure in configuration $\{7 \times 14\}$ (Figure 6.2-(g)). The straightforward explanation for this case is that HEAD-DT optimises its solutions according to the F-Measure, even at the expense of accuracy. In the remaining scenarios, HEAD-DT always outperforms REPTree with statistical significance, which is not the case of CART and C4.5. In fact, CART and C4.5 are only able to outperform REPTree with statistical significance in the F-Measure evaluation in configuration $\{7 \times 14\}$ (Figure 6.2-(h)), suggesting once again that HEAD-DT should be preferred over any of the baseline methods. 


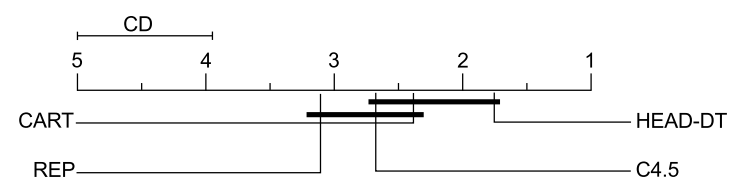

(a) Accuracy rank for $\{1 \times 20\}$.

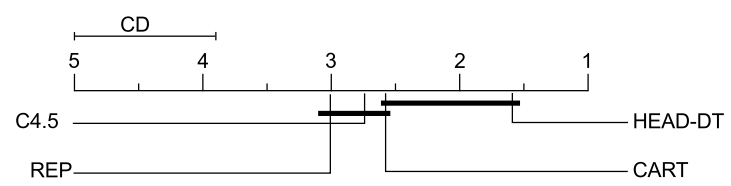

(c) Accuracy rank for $\{3 \times 18\}$.

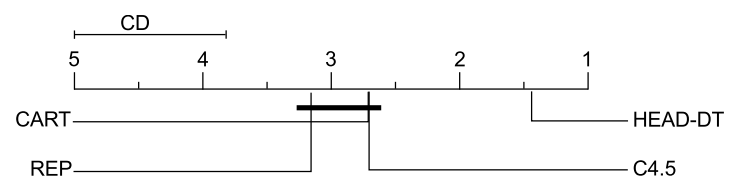

(e) Accuracy rank for $\{5 \times 16\}$.

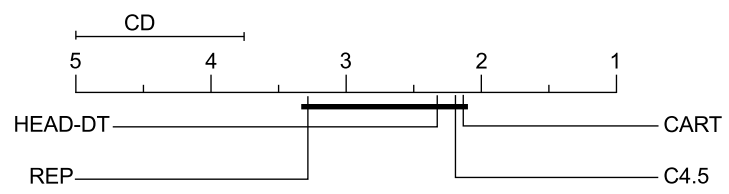

(g) Accuracy rank for $\{7 \times 14\}$.

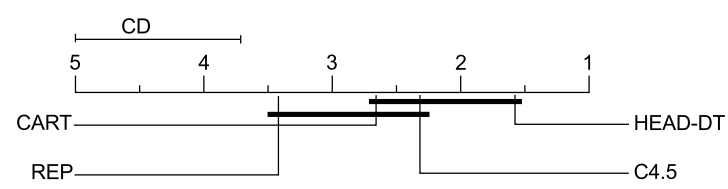

(i) Accuracy rank for $\{9 \times 12\}$.

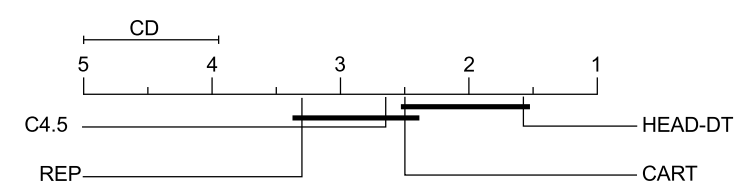

(b) F-Measure rank for $\{1 \times 20\}$.

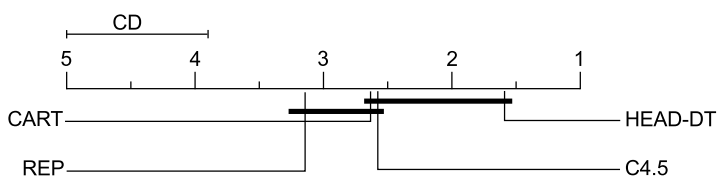

(d) F-Measure rank for $\{3 \times 18\}$.

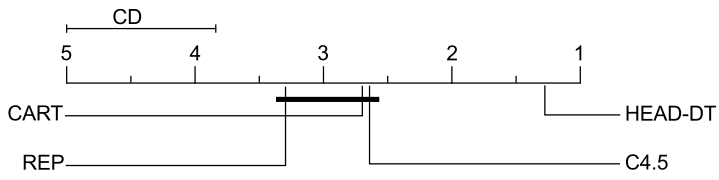

(f) F-Measure rank for $\{5 \times 16\}$.

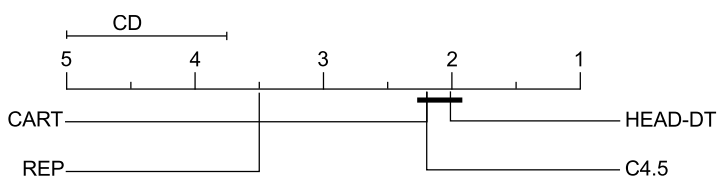

(h) F-Measure rank for $\{7 \times 14\}$.

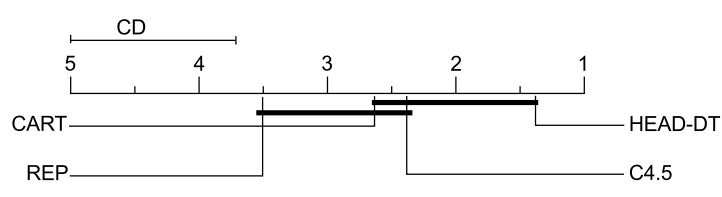

(j) F-Measure rank for $\{9 \times 12\}$.

Figure 6.2: Critical diagrams for the gene expression data. 
As a final remark, considering the results of the four algorithms for all the 10 combinations of experimental configurations and performance measures as a whole, as summarized in Table 6.13, the decision-tree algorithm automatically designed by HEAD-DT obtained the best rank among the four algorithms in 9 out of the 10 rows of Table 6.13. Clearly, if the four algorithms had the same predictive performance (so that each algorithm would have a $25 \%$ probability of being the winner), the probability that the algorithm designed by HEAD-DT would be the winner in 9 out of 10 cases would be very small, so there is a high confidence that the results obtained by HEAD-DT are statistically valid as a whole.

\subsubsection{The Heterogeneous Approach}

In this set of experiments, we investigate the predictive performance of an automatically-designed decision-tree induction algorithm tailored to a variety of distinct data sets. The goal is to evolve an algorithm capable of being robust across different data sets. For such, we make use of 67 publicly-available data sets from the UCI machine-learning repository ${ }^{3}$ (Frank and Asuncion, 2010) (see Table 6.14). As in the homogeneous approach, we also randomly divided the 67 data sets into two groups: parameter optimisation and experiments. The 27 data sets in the parameter optimisation group are used for tuning the evolution parameters of HEAD-DT. The remaining 40 data sets from the experiments group are used for evaluating the performance of the algorithm automatically designed by HEAD-DT.

Following the selection methodology previously presented, the 27 parameter optimisation data sets are arranged in 5 different experimental configurations \{\#training sets, \#test sets $\}:\{1 \times 26\}$, $\{3 \times 24\},\{5 \times 22\},\{7 \times 20\}$, and $\{9 \times 18\}$. Similarly, the 40 data sets in the experiments group are arranged in also 5 different experimental configurations $\{\#$ training sets, \#test sets $\}:\{1 \times 39\}$, $\{3 \times 37\},\{5 \times 35\},\{7 \times 33\}$, and $\{9 \times 31\}$. Table 6.15 presents the randomly selected data sets according to the configurations detailed above.

\section{Parameter Optimisation}

Table 6.16 presents the results of the tuning experiments. We present the average ranking of each version of HEAD-DT (H- $p$ ) in the corresponding experimental configuration. For instance,

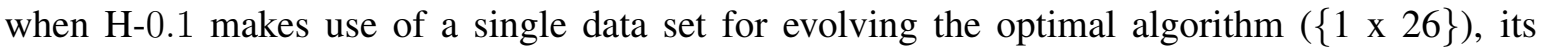
predictive performance in the remaining 26 data sets gives $\mathrm{H}-0.1$ the average rank position of 5.88 for the accuracy of its corresponding decision trees, and 5.96 for the F-Measure.

The Friedman and Nemenyi tests indicated statistically significant differences between the 9 distinct versions of HEAD-DT within each configuration. Depending on the configuration, different versions of HEAD-DT are outperformed by others with statistical significance. The only version of HEAD-DT that is not outperformed by any other version with statistical significance

\footnotetext{
${ }^{3}$ http://archive.ics.uci.edu/ml/
} 
Table 6.14: Summary of the 67 UCI data sets.

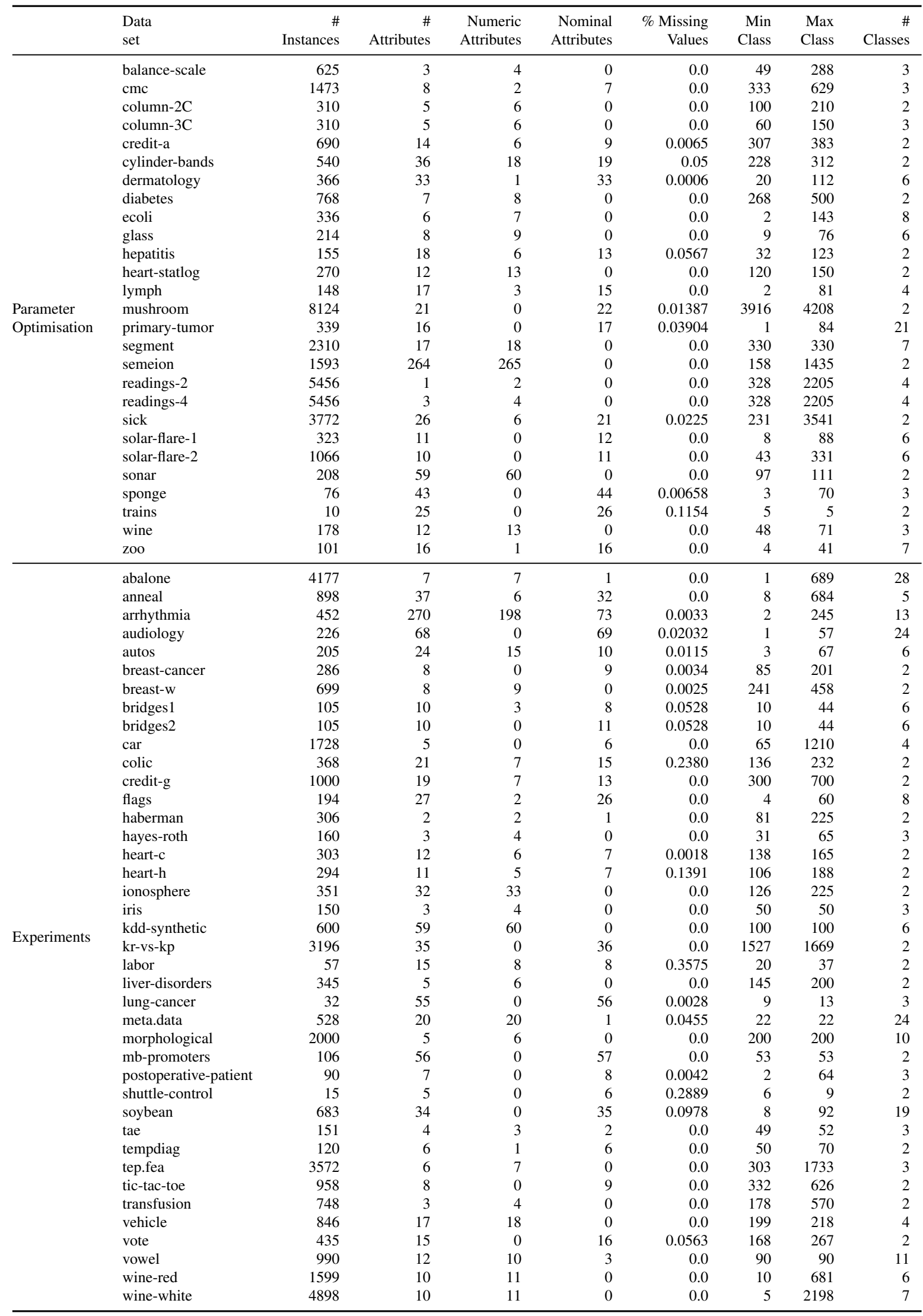


Table 6.15: Meta-training and meta-test configurations for the UCI data.

\begin{tabular}{|c|c|c|c|c|c|c|c|c|c|}
\hline \multicolumn{10}{|c|}{ Data sets for parameter optimisation } \\
\hline \multicolumn{2}{|c|}{$1 \times 26$} & \multicolumn{2}{|c|}{$3 \times 24$} & \multicolumn{2}{|c|}{$5 \times 22$} & \multicolumn{2}{|c|}{$7 \times 20$} & \multicolumn{2}{|c|}{$9 \times 18$} \\
\hline Meta-Training & Meta-Test & Meta-Training & Meta-Test & Meta-Training & Meta-Test & Meta-Training & Meta-Test & Meta-Training & Meta-Test \\
\hline trains & $\begin{array}{l}\text { balance-scale } \\
\text { cmc } \\
\text { column_2C } \\
\text { column_3C } \\
\text { credit-a } \\
\text { cylinder-bands } \\
\text { dermatology } \\
\text { ecoli } \\
\text { glass } \\
\text { heart-statlog } \\
\text { hepatitis } \\
\text { lymph } \\
\text { mushroom } \\
\text { diabetes } \\
\text { primary-tumor } \\
\text { segment } \\
\text { semeion } \\
\text { readings-2 } \\
\text { readings-4 } \\
\text { sick } \\
\text { solar-flare } \\
\text { solar-flare2 } \\
\text { sonar } \\
\text { sponge } \\
\text { wine } \\
\text { zoo }\end{array}$ & $\begin{array}{r}\text { trains } \\
\text { zoo } \\
\text { wine }\end{array}$ & $\begin{array}{l}\text { balance-scale } \\
\text { cmc } \\
\text { column_2C } \\
\text { column_3C } \\
\text { credit-a } \\
\text { cylinder-bands } \\
\text { dermatology } \\
\text { ecoli } \\
\text { glass } \\
\text { heart-statlog } \\
\text { hepatitis } \\
\text { lymph } \\
\text { mushroom } \\
\text { diabetes } \\
\text { primary-tumor } \\
\text { segment } \\
\text { semeion } \\
\text { readings-2 } \\
\text { readings-4 } \\
\text { sick } \\
\text { solar-flare } \\
\text { solar-flare2 } \\
\text { sonar } \\
\text { sponge }\end{array}$ & $\begin{array}{r}\text { trains } \\
\text { zoo } \\
\text { wine } \\
\text { column_2C } \\
\text { glass }\end{array}$ & $\begin{array}{l}\text { balance-scale } \\
\text { cmc } \\
\text { column_3C } \\
\text { credit-a } \\
\text { cylinder-bands } \\
\text { dermatology } \\
\text { ecoli } \\
\text { heart-statlog } \\
\text { hepatitis } \\
\text { lymph } \\
\text { mushroom } \\
\text { diabetes } \\
\text { primary-tumor } \\
\text { segment } \\
\text { semeion } \\
\text { readings-2 } \\
\text { readings-4 } \\
\text { sick } \\
\text { solar-flare } \\
\text { solar-flare2 } \\
\text { sonar } \\
\text { sponge }\end{array}$ & $\begin{array}{r}\text { trains } \\
\text { zoo } \\
\text { wine } \\
\text { column_2C } \\
\text { glass } \\
\text { balance-scale } \\
\text { hepatitis }\end{array}$ & $\begin{array}{l}\text { cmc } \\
\text { column_3C } \\
\text { credit-a } \\
\text { cylinder-bands } \\
\text { dermatology } \\
\text { ecoli } \\
\text { heart-statlog } \\
\text { lymph } \\
\text { mushroom } \\
\text { diabetes } \\
\text { primary-tumor } \\
\text { segment } \\
\text { semeion } \\
\text { readings-2 } \\
\text { readings-4 } \\
\text { sick } \\
\text { solar-flare } \\
\text { solar-flare2 } \\
\text { sonar } \\
\text { sponge }\end{array}$ & $\begin{array}{r}\text { trains } \\
\text { zoo } \\
\text { wine } \\
\text { column_2C } \\
\text { glass } \\
\text { balance-scale } \\
\text { hepatitis } \\
\text { heart-statlog } \\
\text { lymph }\end{array}$ & $\begin{array}{l}\text { cmc } \\
\text { column_3C } \\
\text { credit-a } \\
\text { cylinder-band } \\
\text { dermatology } \\
\text { ecoli } \\
\text { mushroom } \\
\text { diabetes } \\
\text { primary-tumo } \\
\text { segment } \\
\text { semeion } \\
\text { readings-2 } \\
\text { readings-4 } \\
\text { sick } \\
\text { solar-flare } \\
\text { solar-flare2 } \\
\text { sonar } \\
\text { sponge }\end{array}$ \\
\hline & \multicolumn{4}{|c|}{ Data sets for the experimental analysi } & \multirow{2}{*}{\multicolumn{2}{|c|}{$9 \times 31$}} \\
\hline & & & & & & & & & \\
\hline Meta-Training & Meta-Test & Meta-Training & Meta-Test & Meta-Training & Meta-Test & Meta-Training & Meta-Test & Meta-Training & Meta-Test \\
\hline hayes & $\begin{array}{l}\text { abalone } \\
\text { anneal } \\
\text { arrhythmia } \\
\text { audiology } \\
\text { autos } \\
\text { breast-cancer } \\
\text { breast-cancer } \\
\text { bridges1 } \\
\text { bridges2 } \\
\text { car } \\
\text { colic } \\
\text { credit-g } \\
\text { flags } \\
\text { haberman } \\
\text { heart-c } \\
\text { heart-h } \\
\text { ionosphere } \\
\text { iris } \\
\text { kdd-synthetic } \\
\text { kr-vs-kp } \\
\text { labor } \\
\text { liver-disorders } \\
\text { lung-cancer } \\
\text { mb-promoters } \\
\text { meta.data } \\
\text { morphological } \\
\text { postoperative } \\
\text { shuttle-control } \\
\text { soybean } \\
\text { tae } \\
\text { tempdiag } \\
\text { tep.fea } \\
\text { tic-tac-toe } \\
\text { transfusion } \\
\text { vehicle } \\
\text { vote } \\
\text { vowel } \\
\text { wine-red } \\
\text { wine-white }\end{array}$ & $\begin{array}{l}\text { hayes } \\
\text { labor } \\
\text { tae }\end{array}$ & $\begin{array}{l}\text { abalone } \\
\text { anneal } \\
\text { arrhythmia } \\
\text { audiology } \\
\text { autos } \\
\text { breast-cancer } \\
\text { breast-cancer } \\
\text { bridges1 } \\
\text { bridges2 } \\
\text { car } \\
\text { colic } \\
\text { credit-g } \\
\text { flags } \\
\text { haberman } \\
\text { heart-c } \\
\text { heart-h } \\
\text { ionosphere } \\
\text { iris } \\
\text { kdd-synthetic } \\
\text { kr-vs-kp } \\
\text { liver-disorders } \\
\text { lung-cancer } \\
\text { mb-promoters } \\
\text { meta.data } \\
\text { morphological } \\
\text { postoperative } \\
\text { shuttle-control } \\
\text { soybean } \\
\text { tempdiag } \\
\text { tep.fea } \\
\text { tic-tac-toe } \\
\text { transfusion } \\
\text { vehicle } \\
\text { vote } \\
\text { vowel } \\
\text { wine-red } \\
\text { wine-white }\end{array}$ & $\begin{array}{r}\text { hayes } \\
\text { labor } \\
\text { tae } \\
\text { iris } \\
\text { haberman }\end{array}$ & $\begin{array}{l}\text { abalone } \\
\text { anneal } \\
\text { arrhythmia } \\
\text { audiology } \\
\text { autos } \\
\text { breast-cancer } \\
\text { breast-cancer } \\
\text { bridges1 } \\
\text { bridges2 } \\
\text { car } \\
\text { colic } \\
\text { credit-g } \\
\text { flags } \\
\text { heart-c } \\
\text { heart-h } \\
\text { ionosphere } \\
\text { kdd-synthetic } \\
\text { kr-vs-kp } \\
\text { liver-disorders } \\
\text { lung-cancer } \\
\text { mb-promoters } \\
\text { meta.data } \\
\text { morphological } \\
\text { postoperative } \\
\text { shuttle-control } \\
\text { soybean } \\
\text { tempdiag } \\
\text { tep.fea } \\
\text { tic-tac-toe } \\
\text { transfusion } \\
\text { vehicle } \\
\text { vote } \\
\text { vowel } \\
\text { wine-red } \\
\text { wine-white }\end{array}$ & $\begin{array}{r}\text { hayes } \\
\text { labor } \\
\text { tae } \\
\text { iris } \\
\text { haberman } \\
\text { postoperative } \\
\text { bridges1 }\end{array}$ & $\begin{array}{l}\text { abalone } \\
\text { anneal } \\
\text { arrhythmia } \\
\text { audiology } \\
\text { autos } \\
\text { breast-cancer } \\
\text { breast-cancer } \\
\text { bridges2 } \\
\text { car } \\
\text { colic } \\
\text { credit-g } \\
\text { flags } \\
\text { heart-c } \\
\text { heart-h } \\
\text { ionosphere } \\
\text { kdd-synthetic } \\
\text { kr-vs-kp } \\
\text { liver-disorders } \\
\text { lung-cancer } \\
\text { mb-promoters } \\
\text { meta.data } \\
\text { morphological } \\
\text { shuttle-control } \\
\text { soybean } \\
\text { tempdiag } \\
\text { tep.fea } \\
\text { tic-tac-toe } \\
\text { transfusion } \\
\text { vehicle } \\
\text { vote } \\
\text { vowel } \\
\text { wine-red } \\
\text { wine-white }\end{array}$ & $\begin{array}{r}\text { hayes } \\
\text { labor } \\
\text { tae } \\
\text { iris } \\
\text { haberman } \\
\text { postoperative } \\
\text { bridges } 1 \\
\text { tempdiag } \\
\text { lung-cancer }\end{array}$ & $\begin{array}{l}\text { abalone } \\
\text { anneal } \\
\text { arrhythmia } \\
\text { audiology } \\
\text { autos } \\
\text { breast-cancer } \\
\text { breast-cancer } \\
\text { bridges2 } \\
\text { car } \\
\text { colic } \\
\text { credit-g } \\
\text { flags } \\
\text { heart-c } \\
\text { heart-h } \\
\text { ionosphere } \\
\text { kdd-synthetic } \\
\text { kr-vs-kp } \\
\text { liver-disorders } \\
\text { mb-promoters } \\
\text { meta.data } \\
\text { morphological } \\
\text { shuttle-contro } \\
\text { soybean } \\
\text { tep.fea } \\
\text { tic-tac-toe } \\
\text { transfusion } \\
\text { vehicle } \\
\text { vote } \\
\text { vowel } \\
\text { wine-red } \\
\text { wine-white }\end{array}$ \\
\hline
\end{tabular}


Table 6.16: Results of the tuning experiments for the heterogeneous approach.

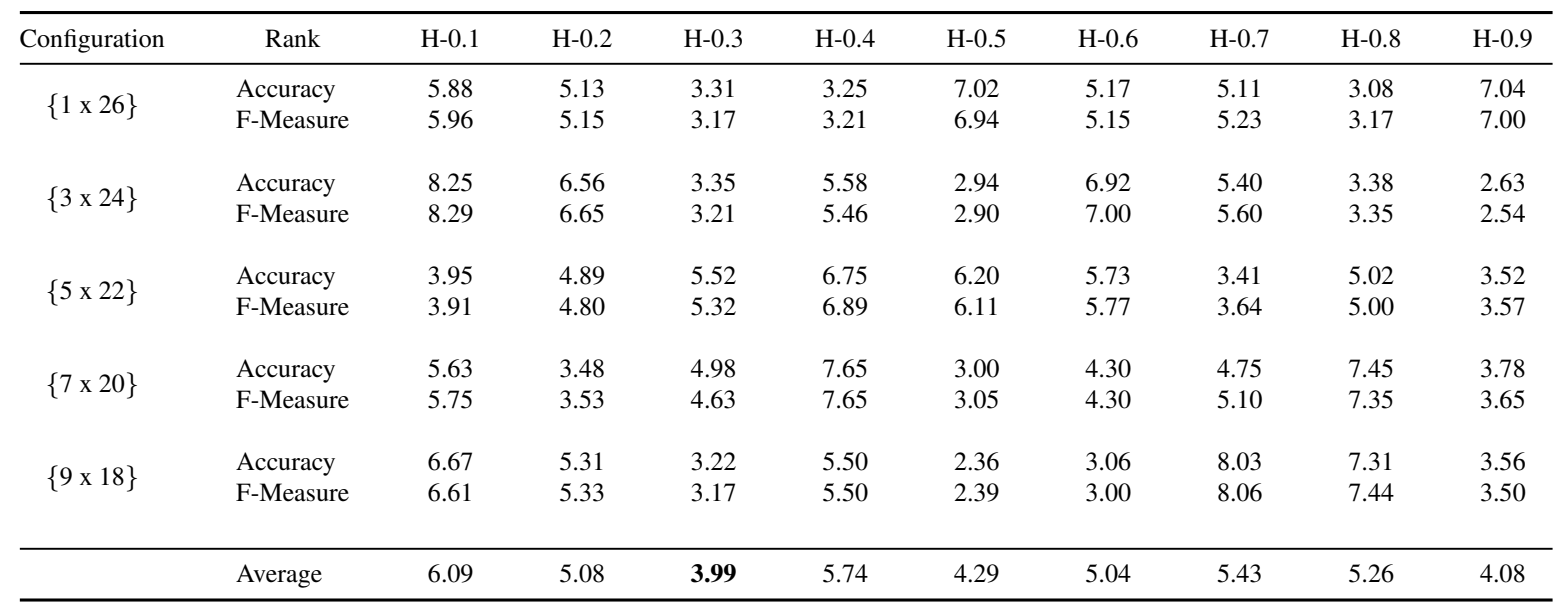

across all configurations is HEAD-0.3. Indeed, when we calculate the average of the average ranks for each $\mathrm{H}-p$ version across the distinct configurations and evaluation measures, HEAD-0.3 provides the lowest average of average ranks (3.99). Hence, HEAD-0.3 is the selected version to be executed over the 40 data sets in the experiments group, and it is henceforth referred simply as HEAD-DT.

\section{Results}

Tables 6.17-6.21 show average values of accuracy and F-Measure obtained by HEAD-DT, CART, C4.5, and REPTree in configurations $\{1 \times 39\},\{3 \times 37\},\{5 \times 35\},\{7 \times 33\}$, and $\{9 \times 31\}$. At the bottom of each table, we present the average rank position of each method. The lower the ranking, the better the method.

The first experiment is regarding configuration $\{1 \times 39\}$. Table 6.17 shows the result for this configuration considering accuracy (Table 6.17-(a)) and F-Measure (Table 6.17-(b)). Note that HEAD-DT and C4.5 are both the best performing method with respect to accuracy, with an average rank of 2.24, followed by CART (2.36) and REPTree (3.15). Considering the F-Measure, C4.5 has a slight edge over HEAD-DT (2.15 to 2.18), followed once again by CART (2.46) and REPTree (3.21).

The next experiment concerns configuration $\left\{\begin{array}{lll}3 \times 37\end{array}\right\}$, whose results are presented in Table 6.18. In the accuracy analysis, C4.5 and CART are the first and second best-ranked algorithms, respectively, followed by HEAD-DT and REPTree. By looking at the F-Measure values, HEAD-DT replaces CART as the second best-ranked method. Note that this is not unusual, since HEAD-DT employs the average F-Measure as its fitness function, and thus optimises its algorithms in order for them to score the best possible F-Measure values. C4.5 keeps its place as the best-performing method, whereas REPTree is again the worst-performing method among the four algorithms, regardless of the evaluation measure. 
Table 6.17: Results for the $\{1 \times 39\}$ configuration.

(a) Accuracy results.

\begin{tabular}{|c|c|c|c|c|}
\hline Data Set & HEAD & CART & C4.5 & REP \\
\hline abalone & $0.36 \pm 0.10$ & $0.26 \pm 0.02$ & $0.22 \pm 0.02$ & $0.26 \pm 0.02$ \\
\hline anneal & $0.92 \pm 0.05$ & $0.98 \pm 0.01$ & $0.99 \pm 0.01$ & $0.98 \pm 0.02$ \\
\hline arrhythmia & $0.66 \pm 0.08$ & $0.71 \pm 0.05$ & $0.66 \pm 0.05$ & $0.67 \pm 0.06$ \\
\hline audiology & $0.70 \pm 0.06$ & $0.74 \pm 0.05$ & $0.78 \pm 0.07$ & $0.74 \pm 0.08$ \\
\hline autos & $0.80 \pm 0.01$ & $0.78 \pm 0.10$ & $0.86 \pm 0.06$ & $0.65 \pm 0.08$ \\
\hline breast-cancer & $0.73 \pm 0.02$ & $0.69 \pm 0.04$ & $0.75 \pm 0.08$ & $0.69 \pm 0.05$ \\
\hline bridges1 & $0.70 \pm 0.03$ & $0.53 \pm 0.09$ & $0.58 \pm 0.11$ & $0.40 \pm 0.15$ \\
\hline bridges2 & $0.64 \pm 0.07$ & $0.54 \pm 0.08$ & $0.58 \pm 0.13$ & $0.40 \pm 0.15$ \\
\hline car & $0.86 \pm 0.07$ & $0.97 \pm 0.02$ & $0.93 \pm 0.02$ & $0.89 \pm 0.02$ \\
\hline heart-c & $0.83 \pm 0.02$ & $0.81 \pm 0.04$ & $0.77 \pm 0.09$ & $0.77 \pm 0.08$ \\
\hline flags & $0.71 \pm 0.04$ & $0.61 \pm 0.10$ & $0.63 \pm 0.05$ & $0.62 \pm 0.10$ \\
\hline credit-g & $0.78 \pm 0.03$ & $0.73 \pm 0.04$ & $0.71 \pm 0.03$ & $0.72 \pm 0.06$ \\
\hline colic & $0.74 \pm 0.07$ & $0.85 \pm 0.08$ & $0.86 \pm 0.06$ & $0.83 \pm 0.06$ \\
\hline haberman & $0.77 \pm 0.01$ & $0.75 \pm 0.04$ & $0.73 \pm 0.09$ & $0.73 \pm 0.07$ \\
\hline heart-h & $0.83 \pm 0.02$ & $0.77 \pm 0.06$ & $0.80 \pm 0.08$ & $0.80 \pm 0.09$ \\
\hline ionosphere & $0.90 \pm 0.03$ & $0.89 \pm 0.03$ & $0.89 \pm 0.05$ & $0.91 \pm 0.02$ \\
\hline iris & $0.96 \pm 0.01$ & $0.93 \pm 0.05$ & $0.94 \pm 0.07$ & $0.94 \pm 0.05$ \\
\hline kdd-synthetic & $0.93 \pm 0.03$ & $0.88 \pm 0.04$ & $0.91 \pm 0.04$ & $0.88 \pm 0.03$ \\
\hline kr-vs-kp & $0.91 \pm 0.06$ & $0.99 \pm 0.01$ & $0.99 \pm 0.01$ & $0.99 \pm 0.01$ \\
\hline labor & $0.79 \pm 0.06$ & $0.81 \pm 0.17$ & $0.79 \pm 0.13$ & $0.82 \pm 0.21$ \\
\hline liver-disorders & $0.77 \pm 0.04$ & $0.67 \pm 0.09$ & $0.67 \pm 0.05$ & $0.65 \pm 0.05$ \\
\hline lung-cancer & $0.66 \pm 0.03$ & $0.51 \pm 0.33$ & $0.45 \pm 0.27$ & $0.54 \pm 0.17$ \\
\hline meta.data & $0.11 \pm 0.02$ & $0.05 \pm 0.03$ & $0.04 \pm 0.03$ & $0.04 \pm 0.00$ \\
\hline morphological & $0.73 \pm 0.03$ & $0.72 \pm 0.04$ & $0.72 \pm 0.02$ & $0.72 \pm 0.03$ \\
\hline mb-promoters & $0.78 \pm 0.07$ & $0.72 \pm 0.14$ & $0.80 \pm 0.13$ & $0.77 \pm 0.15$ \\
\hline postoperative-patient & $0.69 \pm 0.01$ & $0.71 \pm 0.06$ & $0.70 \pm 0.05$ & $0.69 \pm 0.09$ \\
\hline shuttle-control & $0.57 \pm 0.04$ & $0.65 \pm 0.34$ & $0.65 \pm 0.34$ & $0.65 \pm 0.34$ \\
\hline soybean & $0.69 \pm 0.20$ & $0.92 \pm 0.04$ & $0.92 \pm 0.03$ & $0.84 \pm 0.05$ \\
\hline tae & $0.64 \pm 0.03$ & $0.51 \pm 0.12$ & $0.60 \pm 0.11$ & $0.47 \pm 0.12$ \\
\hline tempdiag & $0.97 \pm 0.04$ & $1.00 \pm 0.00$ & $1.00 \pm 0.00$ & $1.00 \pm 0.00$ \\
\hline tep.fea & $0.65 \pm 0.00$ & $0.65 \pm 0.02$ & $0.65 \pm 0.02$ & $0.65 \pm 0.02$ \\
\hline tic-tac-toe & $0.83 \pm 0.08$ & $0.94 \pm 0.02$ & $0.86 \pm 0.03$ & $0.86 \pm 0.03$ \\
\hline transfusion & $0.80 \pm 0.01$ & $0.79 \pm 0.03$ & $0.78 \pm 0.02$ & $0.78 \pm 0.02$ \\
\hline vehicle & $0.78 \pm 0.04$ & $0.72 \pm 0.04$ & $0.74 \pm 0.04$ & $0.71 \pm 0.04$ \\
\hline vote & $0.95 \pm 0.00$ & $0.97 \pm 0.02$ & $0.97 \pm 0.02$ & $0.95 \pm 0.03$ \\
\hline vowel & $0.72 \pm 0.16$ & $0.82 \pm 0.04$ & $0.83 \pm 0.03$ & $0.70 \pm 0.04$ \\
\hline wine-red & $0.67 \pm 0.06$ & $0.63 \pm 0.02$ & $0.61 \pm 0.03$ & $0.60 \pm 0.03$ \\
\hline wine-white & $0.62 \pm 0.10$ & $0.58 \pm 0.02$ & $0.61 \pm 0.03$ & $0.56 \pm 0.02$ \\
\hline breast-w & $0.94 \pm 0.02$ & $0.95 \pm 0.02$ & $0.95 \pm 0.02$ & $0.94 \pm 0.03$ \\
\hline Average Rank & 2.24 & 2.36 & 2.24 & 3.15 \\
\hline
\end{tabular}

(b) F-Measure results.

\begin{tabular}{|c|c|c|c|c|}
\hline Data Set & HEAD-DT & CART & C 4.5 & REP \\
\hline abalone & $0.34 \pm 0.11$ & $0.23 \pm 0.02$ & $0.21 \pm 0.02$ & $0.24 \pm 0.02$ \\
\hline anneal & $0.90 \pm 0.08$ & $0.98 \pm 0.01$ & $0.98 \pm 0.01$ & $0.98 \pm 0.02$ \\
\hline arrhythmia & $0.60 \pm 0.11$ & $0.67 \pm 0.06$ & $0.65 \pm 0.06$ & $0.63 \pm 0.07$ \\
\hline audiology & $0.66 \pm 0.06$ & $0.71 \pm 0.05$ & $0.75 \pm 0.08$ & $0.70 \pm 0.09$ \\
\hline autos & $0.80 \pm 0.01$ & $0.77 \pm 0.10$ & $0.85 \pm 0.07$ & $0.62 \pm 0.07$ \\
\hline breast-cancer & $0.71 \pm 0.03$ & $0.63 \pm 0.05$ & $0.70 \pm 0.11$ & $0.62 \pm 0.06$ \\
\hline breast-w & $0.94 \pm 0.02$ & $0.95 \pm 0.02$ & $0.95 \pm 0.02$ & $0.94 \pm 0.03$ \\
\hline bridges1 & $0.68 \pm 0.04$ & $0.45 \pm 0.06$ & $0.52 \pm 0.11$ & $0.29 \pm 0.11$ \\
\hline bridges2 & $0.62 \pm 0.08$ & $0.43 \pm 0.05$ & $0.51 \pm 0.11$ & $0.29 \pm 0.11$ \\
\hline car & $0.85 \pm 0.08$ & $0.97 \pm 0.02$ & $0.93 \pm 0.02$ & $0.89 \pm 0.02$ \\
\hline heart-c & $0.83 \pm 0.02$ & $0.80 \pm 0.04$ & $0.76 \pm 0.09$ & $0.77 \pm 0.08$ \\
\hline flags & $0.70 \pm 0.05$ & $0.57 \pm 0.10$ & $0.61 \pm 0.05$ & $0.58 \pm 0.10$ \\
\hline credit-g & $0.77 \pm 0.04$ & $0.71 \pm 0.04$ & $0.70 \pm 0.02$ & $0.70 \pm 0.05$ \\
\hline colic & $0.72 \pm 0.09$ & $0.84 \pm 0.08$ & $0.85 \pm 0.07$ & $0.83 \pm 0.07$ \\
\hline haberman & $0.75 \pm 0.01$ & $0.66 \pm 0.06$ & $0.69 \pm 0.10$ & $0.68 \pm 0.08$ \\
\hline heart-h & $0.83 \pm 0.02$ & $0.76 \pm 0.06$ & $0.80 \pm 0.07$ & $0.79 \pm 0.09$ \\
\hline ionosphere & $0.90 \pm 0.04$ & $0.89 \pm 0.03$ & $0.88 \pm 0.05$ & $0.91 \pm 0.02$ \\
\hline iris & $0.96 \pm 0.01$ & $0.93 \pm 0.06$ & $0.94 \pm 0.07$ & $0.94 \pm 0.05$ \\
\hline kdd-synthetic & $0.93 \pm 0.03$ & $0.88 \pm 0.04$ & $0.91 \pm 0.04$ & $0.87 \pm 0.04$ \\
\hline kr-vs-kp & $0.91 \pm 0.06$ & $0.99 \pm 0.01$ & $0.99 \pm 0.01$ & $0.99 \pm 0.01$ \\
\hline labor & $0.76 \pm 0.09$ & $0.80 \pm 0.17$ & $0.78 \pm 0.12$ & $0.82 \pm 0.21$ \\
\hline liver-disorders & $0.77 \pm 0.04$ & $0.66 \pm 0.09$ & $0.66 \pm 0.05$ & $0.63 \pm 0.05$ \\
\hline lung-cancer & $0.65 \pm 0.04$ & $0.42 \pm 0.32$ & $0.35 \pm 0.29$ & $0.42 \pm 0.19$ \\
\hline meta.data & $0.10 \pm 0.03$ & $0.02 \pm 0.01$ & $0.02 \pm 0.02$ & $0.00 \pm 0.00$ \\
\hline morphological & $0.71 \pm 0.04$ & $0.70 \pm 0.04$ & $0.70 \pm 0.02$ & $0.70 \pm 0.03$ \\
\hline mb-promoters & $0.78 \pm 0.07$ & $0.71 \pm 0.14$ & $0.79 \pm 0.14$ & $0.76 \pm 0.15$ \\
\hline postoperative-patient & $0.64 \pm 0.02$ & $0.59 \pm 0.08$ & $0.59 \pm 0.07$ & $0.58 \pm 0.09$ \\
\hline shuttle-control & $0.55 \pm 0.06$ & $0.57 \pm 0.39$ & $0.57 \pm 0.39$ & $0.57 \pm 0.39$ \\
\hline soybean & $0.66 \pm 0.21$ & $0.91 \pm 0.04$ & $0.92 \pm 0.04$ & $0.82 \pm 0.06$ \\
\hline tae & $0.64 \pm 0.03$ & $0.49 \pm 0.15$ & $0.59 \pm 0.12$ & $0.45 \pm 0.12$ \\
\hline tempdiag & $0.97 \pm 0.04$ & $1.00 \pm 0.00$ & $1.00 \pm 0.00$ & $1.00 \pm 0.00$ \\
\hline tep.fea & $0.61 \pm 0.00$ & $0.61 \pm 0.02$ & $0.61 \pm 0.02$ & $0.61 \pm 0.02$ \\
\hline tic-tac-toe & $0.83 \pm 0.09$ & $0.94 \pm 0.02$ & $0.86 \pm 0.03$ & $0.86 \pm 0.03$ \\
\hline transfusion & $0.78 \pm 0.01$ & $0.76 \pm 0.03$ & $0.77 \pm 0.03$ & $0.76 \pm 0.02$ \\
\hline vehicle & $0.77 \pm 0.04$ & $0.71 \pm 0.05$ & $0.73 \pm 0.04$ & $0.70 \pm 0.04$ \\
\hline vote & $0.95 \pm 0.00$ & $0.97 \pm 0.02$ & $0.97 \pm 0.02$ & $0.95 \pm 0.03$ \\
\hline vowel & $0.71 \pm 0.17$ & $0.82 \pm 0.04$ & $0.83 \pm 0.03$ & $0.70 \pm 0.04$ \\
\hline wine-red & $0.66 \pm 0.07$ & $0.61 \pm 0.02$ & $0.61 \pm 0.03$ & $0.58 \pm 0.03$ \\
\hline wine-white & $0.60 \pm 0.12$ & $0.58 \pm 0.03$ & $0.60 \pm 0.02$ & $0.55 \pm 0.02$ \\
\hline Average Rank & 2.18 & 2.46 & 2.15 & 3.21 \\
\hline
\end{tabular}

Table 6.19 presents the experimental results for configuration $\{5 \times 35\}$. The scenario is similar to the previous configuration, with $\mathrm{C} 4.5$ leading the ranking (average rank values of 2.13 for accuracy and 2.04 for F-Measure). C4.5 is followed by CART (2.19 and 2.27) and HEAD-DT (2.56 and 2.44). Finally, REPTree is in the bottom of the ranking, with average rank of 3.13 (accuracy) and 3.24 (F-Measure).

The experimental results for configuration $\{7 \times 33\}$ show a scenario in which HEAD-DT was capable of generating better algorithms than the remaining baseline methods. Table 6.20-(a), which depicts the accuracy values of each method, indicates that HEAD-DT leads the ranking with an average ranking position of 1.77 - meaning its often the best-performing method in the 33 meta-test data sets. HEAD-DT is followed by C4.5 (2.35), CART (2.50), and REPTree (3.38). Table 6.20-(b) shows that the F-Measure values provide the same ranking positions for all methods, with a clear edge to HEAD-DT (1.71), followed by C4.5 (2.29), CART (2.53), and REPTree (3.47).

Finally, Table 6.21 shows the results for configuration $\{9 \times 31\}$. C4.5 returns to the top of the rank for both accuracy (2.03) and F-Measure (1.96) values. C4.5 is followed by CART (2.26 and 2.32), HEAD-DT (2.55 and 2.48), and REPTree (3.16 and 3.23). 
Table 6.18: Results for the $\{3 \times 37\}$ configuration.

(a) Accuracy results.

\begin{tabular}{|c|c|c|c|c|}
\hline Data Set & HEAD & CART & $\mathrm{C} 4.5$ & REP \\
\hline abalone & $0.27 \pm 0.00$ & $0.26 \pm 0.02$ & $0.22 \pm 0.02$ & $0.26 \pm 0.02$ \\
\hline anneal & $0.98 \pm 0.01$ & $0.98 \pm 0.01$ & $0.99 \pm 0.01$ & $0.98 \pm 0.02$ \\
\hline arrhythmia & $0.66 \pm 0.10$ & $0.71 \pm 0.05$ & $0.66 \pm 0.05$ & $0.67 \pm 0.06$ \\
\hline audiology & $0.73 \pm 0.02$ & $0.74 \pm 0.05$ & $0.78 \pm 0.07$ & $0.74 \pm 0.08$ \\
\hline autos & $0.73 \pm 0.08$ & $0.78 \pm 0.10$ & $0.86 \pm 0.06$ & $0.65 \pm 0.08$ \\
\hline breast-cancer & $0.74 \pm 0.01$ & $0.69 \pm 0.04$ & $0.75 \pm 0.08$ & $0.69 \pm 0.05$ \\
\hline breast-w & $0.94 \pm 0.01$ & $0.95 \pm 0.02$ & $0.95 \pm 0.02$ & $0.94 \pm 0.03$ \\
\hline bridges1 & $0.70 \pm 0.06$ & $0.53 \pm 0.09$ & $0.58 \pm 0.11$ & $0.40 \pm 0.15$ \\
\hline bridges2 & $0.69 \pm 0.05$ & $0.54 \pm 0.08$ & $0.58 \pm 0.13$ & $0.40 \pm 0.15$ \\
\hline car & $0.84 \pm 0.02$ & $0.97 \pm 0.02$ & $0.93 \pm 0.02$ & $0.89 \pm 0.02$ \\
\hline heart-c & $0.81 \pm 0.00$ & $0.81 \pm 0.04$ & $0.77 \pm 0.09$ & $0.77 \pm 0.08$ \\
\hline flags & $0.69 \pm 0.01$ & $0.61 \pm 0.10$ & $0.63 \pm 0.05$ & $0.62 \pm 0.10$ \\
\hline credit-g & $0.74 \pm 0.00$ & $0.73 \pm 0.04$ & $0.71 \pm 0.03$ & $0.72 \pm 0.06$ \\
\hline colic & $0.78 \pm 0.12$ & $0.85 \pm 0.08$ & $0.86 \pm 0.06$ & $0.83 \pm 0.06$ \\
\hline haberman & $0.77 \pm 0.00$ & $0.75 \pm 0.04$ & $0.73 \pm 0.09$ & $0.73 \pm 0.07$ \\
\hline heart-h & $0.80 \pm 0.01$ & $0.77 \pm 0.06$ & $0.80 \pm 0.08$ & $0.80 \pm 0.09$ \\
\hline ionosphere & $0.92 \pm 0.03$ & $0.89 \pm 0.03$ & $0.89 \pm 0.05$ & $0.91 \pm 0.02$ \\
\hline iris & $0.96 \pm 0.00$ & $0.93 \pm 0.05$ & $0.94 \pm 0.07$ & $0.94 \pm 0.05$ \\
\hline kdd-synthetic & $0.95 \pm 0.01$ & $0.88 \pm 0.04$ & $0.91 \pm 0.04$ & $0.88 \pm 0.03$ \\
\hline kr-vs-kp & $0.91 \pm 0.03$ & $0.99 \pm 0.01$ & $0.99 \pm 0.01$ & $0.99 \pm 0.01$ \\
\hline liver-disorders & $0.73 \pm 0.01$ & $0.67 \pm 0.09$ & $0.67 \pm 0.05$ & $0.65 \pm 0.05$ \\
\hline lung-cancer & $0.69 \pm 0.00$ & $0.51 \pm 0.33$ & $0.45 \pm 0.27$ & $0.54 \pm 0.17$ \\
\hline meta.data & $0.08 \pm 0.04$ & $0.05 \pm 0.03$ & $0.04 \pm 0.03$ & $0.04 \pm 0.00$ \\
\hline morphological & $0.71 \pm 0.00$ & $0.72 \pm 0.04$ & $0.72 \pm 0.02$ & $0.72 \pm 0.03$ \\
\hline mb-promoters & $0.88 \pm 0.02$ & $0.72 \pm 0.14$ & $0.80 \pm 0.13$ & $0.77 \pm 0.15$ \\
\hline postoperative-patient & $0.70 \pm 0.02$ & $0.71 \pm 0.06$ & $0.70 \pm 0.05$ & $0.69 \pm 0.09$ \\
\hline shuttle-control & $0.60 \pm 0.02$ & $0.65 \pm 0.34$ & $0.65 \pm 0.34$ & $0.65 \pm 0.34$ \\
\hline soybean & $0.79 \pm 0.06$ & $0.92 \pm 0.04$ & $0.92 \pm 0.03$ & $0.84 \pm 0.05$ \\
\hline tempdiag & $1.00 \pm 0.00$ & $1.00 \pm 0.00$ & $1.00 \pm 0.00$ & $1.00 \pm 0.00$ \\
\hline tep.fea & $0.65 \pm 0.00$ & $0.65 \pm 0.02$ & $0.65 \pm 0.02$ & $0.65 \pm 0.02$ \\
\hline tic-tac-toe & $0.76 \pm 0.04$ & $0.94 \pm 0.02$ & $0.86 \pm 0.03$ & $0.86 \pm 0.03$ \\
\hline transfusion & $0.79 \pm 0.01$ & $0.79 \pm 0.03$ & $0.78 \pm 0.02$ & $0.78 \pm 0.02$ \\
\hline vehicle & $0.74 \pm 0.00$ & $0.72 \pm 0.04$ & $0.74 \pm 0.04$ & $0.71 \pm 0.04$ \\
\hline vote & $0.95 \pm 0.01$ & $0.97 \pm 0.02$ & $0.97 \pm 0.02$ & $0.95 \pm 0.03$ \\
\hline vowel & $0.59 \pm 0.08$ & $0.82 \pm 0.04$ & $0.83 \pm 0.03$ & $0.70 \pm 0.04$ \\
\hline wine-red & $0.59 \pm 0.01$ & $0.63 \pm 0.02$ & $0.61 \pm 0.03$ & $0.60 \pm 0.03$ \\
\hline wine-white & $0.52 \pm 0.01$ & $0.58 \pm 0.02$ & $0.61 \pm 0.03$ & $0.56 \pm 0.02$ \\
\hline Average Rank & 2.45 & 2.26 & 2.19 & 3.11 \\
\hline
\end{tabular}

(b) F-Measure results.

\begin{tabular}{|c|c|c|c|c|}
\hline Data Set & HEAD & CART & C 4.5 & REP \\
\hline abalone & $0.23 \pm 0.00$ & $0.23 \pm 0.02$ & $0.21 \pm 0.02$ & $0.24 \pm 0.02$ \\
\hline anneal & $0.97 \pm 0.01$ & $0.98 \pm 0.01$ & $0.98 \pm 0.01$ & $0.98 \pm 0.02$ \\
\hline arrhythmia & $0.58 \pm 0.17$ & $0.67 \pm 0.06$ & $0.65 \pm 0.06$ & $0.63 \pm 0.07$ \\
\hline audiology & $0.70 \pm 0.02$ & $0.71 \pm 0.05$ & $0.75 \pm 0.08$ & $0.70 \pm 0.09$ \\
\hline autos & $0.73 \pm 0.08$ & $0.77 \pm 0.10$ & $0.85 \pm 0.07$ & $0.62 \pm 0.07$ \\
\hline breast-cancer & $0.72 \pm 0.00$ & $0.63 \pm 0.05$ & $0.70 \pm 0.11$ & $0.62 \pm 0.06$ \\
\hline breast-w & $0.94 \pm 0.01$ & $0.95 \pm 0.02$ & $0.95 \pm 0.02$ & $0.94 \pm 0.03$ \\
\hline bridges1 & $0.68 \pm 0.06$ & $0.45 \pm 0.06$ & $0.52 \pm 0.11$ & $0.29 \pm 0.11$ \\
\hline bridges2 & $0.68 \pm 0.06$ & $0.43 \pm 0.05$ & $0.51 \pm 0.11$ & $0.29 \pm 0.11$ \\
\hline car & $0.83 \pm 0.02$ & $0.97 \pm 0.02$ & $0.93 \pm 0.02$ & $0.89 \pm 0.02$ \\
\hline heart-c & $0.81 \pm 0.00$ & $0.80 \pm 0.04$ & $0.76 \pm 0.09$ & $0.77 \pm 0.08$ \\
\hline flags & $0.68 \pm 0.01$ & $0.57 \pm 0.10$ & $0.61 \pm 0.05$ & $0.58 \pm 0.10$ \\
\hline credit-g & $0.73 \pm 0.00$ & $0.71 \pm 0.04$ & $0.70 \pm 0.02$ & $0.70 \pm 0.05$ \\
\hline colic & $0.72 \pm 0.18$ & $0.84 \pm 0.08$ & $0.85 \pm 0.07$ & $0.83 \pm 0.07$ \\
\hline haberman & $0.75 \pm 0.01$ & $0.66 \pm 0.06$ & $0.69 \pm 0.10$ & $0.68 \pm 0.08$ \\
\hline heart-h & $0.80 \pm 0.01$ & $0.76 \pm 0.06$ & $0.80 \pm 0.07$ & $0.79 \pm 0.09$ \\
\hline ionosphere & $0.92 \pm 0.03$ & $0.89 \pm 0.03$ & $0.88 \pm 0.05$ & $0.91 \pm 0.02$ \\
\hline iris & $0.96 \pm 0.00$ & $0.93 \pm 0.06$ & $0.94 \pm 0.07$ & $0.94 \pm 0.05$ \\
\hline kdd-synthetic & $0.95 \pm 0.01$ & $0.88 \pm 0.04$ & $0.91 \pm 0.04$ & $0.87 \pm 0.04$ \\
\hline kr-vs-kp & $0.91 \pm 0.03$ & $0.99 \pm 0.01$ & $0.99 \pm 0.01$ & $0.99 \pm 0.01$ \\
\hline liver-disorders & $0.72 \pm 0.02$ & $0.66 \pm 0.09$ & $0.66 \pm 0.05$ & $0.63 \pm 0.05$ \\
\hline lung-cancer & $0.69 \pm 0.00$ & $0.42 \pm 0.32$ & $0.35 \pm 0.29$ & $0.42 \pm 0.19$ \\
\hline meta.data & $0.06 \pm 0.03$ & $0.02 \pm 0.01$ & $0.02 \pm 0.02$ & $0.00 \pm 0.00$ \\
\hline morphological & $0.70 \pm 0.00$ & $0.70 \pm 0.04$ & $0.70 \pm 0.02$ & $0.70 \pm 0.03$ \\
\hline mb-promoters & $0.88 \pm 0.02$ & $0.71 \pm 0.14$ & $0.79 \pm 0.14$ & $0.76 \pm 0.15$ \\
\hline postoperative-patient & $0.67 \pm 0.02$ & $0.59 \pm 0.08$ & $0.59 \pm 0.07$ & $0.58 \pm 0.09$ \\
\hline shuttle-control & $0.58 \pm 0.02$ & $0.57 \pm 0.39$ & $0.57 \pm 0.39$ & $0.57 \pm 0.39$ \\
\hline soybean & $0.76 \pm 0.07$ & $0.91 \pm 0.04$ & $0.92 \pm 0.04$ & $0.82 \pm 0.06$ \\
\hline tempdiag & $1.00 \pm 0.00$ & $1.00 \pm 0.00$ & $1.00 \pm 0.00$ & $1.00 \pm 0.00$ \\
\hline tep.fea & $0.61 \pm 0.00$ & $0.61 \pm 0.02$ & $0.61 \pm 0.02$ & $0.61 \pm 0.02$ \\
\hline tic-tac-toe & $0.76 \pm 0.04$ & $0.94 \pm 0.02$ & $0.86 \pm 0.03$ & $0.86 \pm 0.03$ \\
\hline transfusion & $0.77 \pm 0.00$ & $0.76 \pm 0.03$ & $0.77 \pm 0.03$ & $0.76 \pm 0.02$ \\
\hline vehicle & $0.74 \pm 0.00$ & $0.71 \pm 0.05$ & $0.73 \pm 0.04$ & $0.70 \pm 0.04$ \\
\hline vote & $0.95 \pm 0.01$ & $0.97 \pm 0.02$ & $0.97 \pm 0.02$ & $0.95 \pm 0.03$ \\
\hline vowel & $0.58 \pm 0.09$ & $0.82 \pm 0.04$ & $0.83 \pm 0.03$ & $0.70 \pm 0.04$ \\
\hline wine-red & $0.57 \pm 0.01$ & $0.61 \pm 0.02$ & $0.61 \pm 0.03$ & $0.58 \pm 0.03$ \\
\hline wine-white & $0.48 \pm 0.02$ & $0.58 \pm 0.03$ & $0.60 \pm 0.02$ & $0.55 \pm 0.02$ \\
\hline Average Rank & 2.23 & 2.42 & 2.15 & 3.20 \\
\hline
\end{tabular}

Table 6.19: Results for the $\{5 \times 35\}$ configuration.

(a) Accuracy results.

\begin{tabular}{lrrrr}
\hline \multicolumn{1}{c}{ Data Set } & HEAD & CART & C4.5 & REP \\
\hline abalone & $0.27 \pm 0.00$ & $0.26 \pm 0.02$ & $0.22 \pm 0.02$ & $0.26 \pm 0.02$ \\
anneal & $0.97 \pm 0.00$ & $0.98 \pm 0.01$ & $0.99 \pm 0.01$ & $0.98 \pm 0.02$ \\
arrhythmia & $0.58 \pm 0.08$ & $0.71 \pm 0.05$ & $0.66 \pm 0.05$ & $0.67 \pm 0.06$ \\
audiology & $0.76 \pm 0.00$ & $0.74 \pm 0.05$ & $0.78 \pm 0.07$ & $0.74 \pm 0.08$ \\
autos & $0.67 \pm 0.06$ & $0.78 \pm 0.10$ & $0.86 \pm 0.06$ & $0.65 \pm 0.08$ \\
breast-cancer & $0.75 \pm 0.00$ & $0.69 \pm 0.04$ & $0.75 \pm 0.08$ & $0.69 \pm 0.05$ \\
breast-w & $0.93 \pm 0.00$ & $0.95 \pm 0.02$ & $0.95 \pm 0.02$ & $0.94 \pm 0.03$ \\
bridges1 & $0.64 \pm 0.03$ & $0.53 \pm 0.09$ & $0.58 \pm 0.11$ & $0.40 \pm 0.15$ \\
bridges2 & $0.64 \pm 0.03$ & $0.54 \pm 0.08$ & $0.58 \pm 0.13$ & $0.40 \pm 0.15$ \\
car & $0.82 \pm 0.02$ & $0.97 \pm 0.02$ & $0.93 \pm 0.02$ & $0.89 \pm 0.02$ \\
heart-c & $0.81 \pm 0.01$ & $0.81 \pm 0.04$ & $0.77 \pm 0.09$ & $0.77 \pm 0.08$ \\
flags & $0.68 \pm 0.01$ & $0.61 \pm 0.10$ & $0.63 \pm 0.05$ & $0.62 \pm 0.10$ \\
credit-g & $0.75 \pm 0.00$ & $0.73 \pm 0.04$ & $0.71 \pm 0.03$ & $0.72 \pm 0.06$ \\
colic & $0.68 \pm 0.09$ & $0.85 \pm 0.08$ & $0.86 \pm 0.06$ & $0.83 \pm 0.06$ \\
heart-h & $0.77 \pm 0.05$ & $0.77 \pm 0.06$ & $0.80 \pm 0.08$ & $0.80 \pm 0.09$ \\
ionosphere & $0.89 \pm 0.00$ & $0.89 \pm 0.03$ & $0.89 \pm 0.05$ & $0.91 \pm 0.02$ \\
kdd-synthetic & $0.96 \pm 0.00$ & $0.88 \pm 0.04$ & $0.91 \pm 0.04$ & $0.88 \pm 0.03$ \\
kr-v-kp & $0.95 \pm 0.00$ & $0.99 \pm 0.01$ & $0.99 \pm 0.01$ & $0.99 \pm 0.01$ \\
liver-disorders & $0.74 \pm 0.01$ & $0.67 \pm 0.09$ & $0.67 \pm 0.05$ & $0.65 \pm 0.05$ \\
lung-cancer & $0.69 \pm 0.00$ & $0.51 \pm 0.33$ & $0.45 \pm 0.27$ & $0.54 \pm 0.17$ \\
meta.data & $0.04 \pm 0.02$ & $0.05 \pm 0.03$ & $0.04 \pm 0.03$ & $0.04 \pm 0.00$ \\
morphological & $0.70 \pm 0.00$ & $0.72 \pm 0.04$ & $0.72 \pm 0.02$ & $0.72 \pm 0.03$ \\
mb-promoters & $0.86 \pm 0.01$ & $0.72 \pm 0.14$ & $0.80 \pm 0.13$ & $0.77 \pm 0.15$ \\
postoperative-patient & $0.72 \pm 0.02$ & $0.71 \pm 0.06$ & $0.70 \pm 0.05$ & $0.69 \pm 0.09$ \\
shuttle-control & $0.61 \pm 0.01$ & $0.65 \pm 0.34$ & $0.65 \pm 0.34$ & $0.65 \pm 0.34$ \\
soybean & $0.72 \pm 0.02$ & $0.92 \pm 0.04$ & $0.92 \pm 0.03$ & $0.84 \pm 0.05$ \\
tempdiag & $1.00 \pm 0.00$ & $1.00 \pm 0.00$ & $1.00 \pm 0.00$ & $1.00 \pm 0.00$ \\
tep.fea & $0.65 \pm 0.00$ & $0.65 \pm 0.02$ & $0.65 \pm 0.02$ & $0.65 \pm 0.02$ \\
tic-tac-toe & $0.73 \pm 0.03$ & $0.94 \pm 0.02$ & $0.86 \pm 0.03$ & $0.86 \pm 0.03$ \\
transfusion & $0.79 \pm 0.00$ & $0.79 \pm 0.03$ & $0.78 \pm 0.02$ & $0.78 \pm 0.02$ \\
vehicle & $0.74 \pm 0.00$ & $0.72 \pm 0.04$ & $0.74 \pm 0.04$ & $0.71 \pm 0.04$ \\
vote & $0.96 \pm 0.00$ & $0.97 \pm 0.02$ & $0.97 \pm 0.02$ & $0.95 \pm 0.03$ \\
vowel & $0.50 \pm 0.01$ & $0.82 \pm 0.04$ & $0.83 \pm 0.03$ & $0.70 \pm 0.04$ \\
wine-red & $0.60 \pm 0.00$ & $0.63 \pm 0.02$ & $0.61 \pm 0.03$ & $0.60 \pm 0.03$ \\
wine-white & $0.54 \pm 0.00$ & $0.58 \pm 0.02$ & $0.61 \pm 0.03$ & $0.56 \pm 0.02$ \\
\hline Average Rank & 2.56 & 2.19 & $\mathbf{2 . 1 3}$ & 3.13 \\
\hline & & & &
\end{tabular}

(b) F-Measure results.

\begin{tabular}{lrrrr}
\hline \multicolumn{1}{c}{ Data Set } & HEAD & CART & C4.5 & REP \\
\hline abalone & $0.24 \pm 0.00$ & $0.23 \pm 0.02$ & $0.21 \pm 0.02$ & $0.24 \pm 0.02$ \\
anneal & $0.97 \pm 0.00$ & $0.98 \pm 0.01$ & $0.98 \pm 0.01$ & $0.98 \pm 0.02$ \\
arrhythmia & $0.45 \pm 0.13$ & $0.67 \pm 0.06$ & $0.65 \pm 0.06$ & $0.63 \pm 0.07$ \\
audiology & $0.73 \pm 0.00$ & $0.71 \pm 0.05$ & $0.75 \pm 0.08$ & $0.70 \pm 0.09$ \\
autos & $0.67 \pm 0.06$ & $0.77 \pm 0.10$ & $0.85 \pm 0.07$ & $0.62 \pm 0.07$ \\
breast-cancer & $0.73 \pm 0.00$ & $0.63 \pm 0.05$ & $0.70 \pm 0.11$ & $0.62 \pm 0.06$ \\
breast-w & $0.93 \pm 0.00$ & $0.95 \pm 0.02$ & $0.95 \pm 0.02$ & $0.94 \pm 0.03$ \\
bridges1 & $0.63 \pm 0.03$ & $0.45 \pm 0.06$ & $0.52 \pm 0.11$ & $0.29 \pm 0.11$ \\
bridges2 & $0.63 \pm 0.04$ & $0.43 \pm 0.05$ & $0.51 \pm 0.11$ & $0.29 \pm 0.11$ \\
car & $0.81 \pm 0.02$ & $0.97 \pm 0.02$ & $0.93 \pm 0.02$ & $0.89 \pm 0.02$ \\
heart-c & $0.80 \pm 0.01$ & $0.80 \pm 0.04$ & $0.76 \pm 0.09$ & $0.77 \pm 0.08$ \\
flags & $0.67 \pm 0.01$ & $0.57 \pm 0.10$ & $0.61 \pm 0.05$ & $0.58 \pm 0.10$ \\
credit-g & $0.73 \pm 0.00$ & $0.71 \pm 0.04$ & $0.70 \pm 0.02$ & $0.70 \pm 0.05$ \\
colic & $0.57 \pm 0.14$ & $0.84 \pm 0.08$ & $0.85 \pm 0.07$ & $0.83 \pm 0.07$ \\
heart-h & $0.74 \pm 0.07$ & $0.76 \pm 0.06$ & $0.80 \pm 0.07$ & $0.79 \pm 0.09$ \\
ionosphere & $0.89 \pm 0.01$ & $0.89 \pm 0.03$ & $0.88 \pm 0.05$ & $0.91 \pm 0.02$ \\
kdd-synthetic & $0.96 \pm 0.00$ & $0.88 \pm 0.04$ & $0.91 \pm 0.04$ & $0.87 \pm 0.04$ \\
kr-vs-kp & $0.95 \pm 0.00$ & $0.99 \pm 0.01$ & $0.99 \pm 0.01$ & $0.99 \pm 0.01$ \\
liver-disorders & $0.73 \pm 0.01$ & $0.66 \pm 0.09$ & $0.66 \pm 0.05$ & $0.63 \pm 0.05$ \\
lung-cancer & $0.69 \pm 0.00$ & $0.42 \pm 0.32$ & $0.35 \pm 0.29$ & $0.42 \pm 0.19$ \\
meta.data & $0.02 \pm 0.01$ & $0.02 \pm 0.01$ & $0.02 \pm 0.02$ & $0.00 \pm 0.00$ \\
morphological & $0.69 \pm 0.01$ & $0.70 \pm 0.04$ & $0.70 \pm 0.02$ & $0.70 \pm 0.03$ \\
mb-promoters & $0.86 \pm 0.01$ & $0.71 \pm 0.14$ & $0.79 \pm 0.14$ & $0.76 \pm 0.15$ \\
postoperative-patient & $0.69 \pm 0.03$ & $0.59 \pm 0.08$ & $0.59 \pm 0.07$ & $0.58 \pm 0.09$ \\
shuttle-control & $0.57 \pm 0.02$ & $0.57 \pm 0.39$ & $0.57 \pm 0.39$ & $0.57 \pm 0.39$ \\
soybean & $0.68 \pm 0.01$ & $0.91 \pm 0.04$ & $0.92 \pm 0.04$ & $0.82 \pm 0.06$ \\
tempdiag & $1.00 \pm 0.00$ & $1.00 \pm 0.00$ & $1.00 \pm 0.00$ & $1.00 \pm 0.00$ \\
tep.fea & $0.61 \pm 0.00$ & $0.61 \pm 0.02$ & $0.61 \pm 0.02$ & $0.61 \pm 0.02$ \\
tic-tac-toe & $0.72 \pm 0.04$ & $0.94 \pm 0.02$ & $0.86 \pm 0.03$ & $0.86 \pm 0.03$ \\
transfusion & $0.77 \pm 0.00$ & $0.76 \pm 0.03$ & $0.77 \pm 0.03$ & $0.76 \pm 0.02$ \\
vehicle & $0.74 \pm 0.00$ & $0.71 \pm 0.05$ & $0.73 \pm 0.04$ & $0.70 \pm 0.04$ \\
vote & $0.96 \pm 0.00$ & $0.97 \pm 0.02$ & $0.97 \pm 0.02$ & $0.95 \pm 0.03$ \\
vowel & $0.48 \pm 0.02$ & $0.82 \pm 0.04$ & $0.83 \pm 0.03$ & $0.70 \pm 0.04$ \\
wine-red & $0.59 \pm 0.00$ & $0.61 \pm 0.02$ & $0.61 \pm 0.03$ & $0.58 \pm 0.03$ \\
wine-white & $0.51 \pm 0.00$ & $0.58 \pm 0.03$ & $0.60 \pm 0.02$ & $0.55 \pm 0.02$ \\
\hline Average Rank & 2.44 & 2.27 & $\mathbf{2 . 0 4}$ & 3.24 \\
\hline & & & &
\end{tabular}


Table 6.20: Results for the $\{7 \times 33\}$ configuration.

(a) Accuracy results.

\begin{tabular}{|c|c|c|c|c|}
\hline Data Set & HEAD & CART & C4.5 & REP \\
\hline abalone & $0.29 \pm 0.01$ & $0.26 \pm 0.02$ & $0.22 \pm 0.02$ & $0.26 \pm 0.02$ \\
\hline anneal & $0.98 \pm 0.01$ & $0.98 \pm 0.01$ & $0.99 \pm 0.01$ & $0.98 \pm 0.02$ \\
\hline arrhythmia & $0.78 \pm 0.02$ & $0.71 \pm 0.05$ & $0.66 \pm 0.05$ & $0.67 \pm 0.06$ \\
\hline audiology & $0.79 \pm 0.00$ & $0.74 \pm 0.05$ & $0.78 \pm 0.07$ & $0.74 \pm 0.08$ \\
\hline autos & $0.84 \pm 0.04$ & $0.78 \pm 0.10$ & $0.86 \pm 0.06$ & $0.65 \pm 0.08$ \\
\hline breast-cancer & $0.75 \pm 0.00$ & $0.69 \pm 0.04$ & $0.75 \pm 0.08$ & $0.69 \pm 0.05$ \\
\hline breast-w & $0.96 \pm 0.01$ & $0.95 \pm 0.02$ & $0.95 \pm 0.02$ & $0.94 \pm 0.03$ \\
\hline bridges2 & $0.71 \pm 0.01$ & $0.54 \pm 0.08$ & $0.58 \pm 0.13$ & $0.40 \pm 0.15$ \\
\hline car & $0.92 \pm 0.02$ & $0.97 \pm 0.02$ & $0.93 \pm 0.02$ & $0.89 \pm 0.02$ \\
\hline heart-c & $0.83 \pm 0.01$ & $0.81 \pm 0.04$ & $0.77 \pm 0.09$ & $0.77 \pm 0.08$ \\
\hline flags & $0.73 \pm 0.01$ & $0.61 \pm 0.10$ & $0.63 \pm 0.05$ & $0.62 \pm 0.10$ \\
\hline credit-g & $0.76 \pm 0.00$ & $0.73 \pm 0.04$ & $0.71 \pm 0.03$ & $0.72 \pm 0.06$ \\
\hline colic & $0.88 \pm 0.01$ & $0.85 \pm 0.08$ & $0.86 \pm 0.06$ & $0.83 \pm 0.06$ \\
\hline heart-h & $0.83 \pm 0.01$ & $0.77 \pm 0.06$ & $0.80 \pm 0.08$ & $0.80 \pm 0.09$ \\
\hline ionosphere & $0.94 \pm 0.01$ & $0.89 \pm 0.03$ & $0.89 \pm 0.05$ & $0.91 \pm 0.02$ \\
\hline kdd-synthetic & $0.95 \pm 0.00$ & $0.88 \pm 0.04$ & $0.91 \pm 0.04$ & $0.88 \pm 0.03$ \\
\hline kr-vs-kp & $0.96 \pm 0.00$ & $0.99 \pm 0.01$ & $0.99 \pm 0.01$ & $0.99 \pm 0.01$ \\
\hline liver-disorders & $0.75 \pm 0.00$ & $0.67 \pm 0.09$ & $0.67 \pm 0.05$ & $0.65 \pm 0.05$ \\
\hline lung-cancer & $0.71 \pm 0.02$ & $0.51 \pm 0.33$ & $0.45 \pm 0.27$ & $0.54 \pm 0.17$ \\
\hline meta.data & $0.14 \pm 0.02$ & $0.05 \pm 0.03$ & $0.04 \pm 0.03$ & $0.04 \pm 0.00$ \\
\hline morphological & $0.74 \pm 0.01$ & $0.72 \pm 0.04$ & $0.72 \pm 0.02$ & $0.72 \pm 0.03$ \\
\hline mb-promoters & $0.89 \pm 0.01$ & $0.72 \pm 0.14$ & $0.80 \pm 0.13$ & $0.77 \pm 0.15$ \\
\hline shuttle-control & $0.59 \pm 0.02$ & $0.65 \pm 0.34$ & $0.65 \pm 0.34$ & $0.65 \pm 0.34$ \\
\hline soybean & $0.82 \pm 0.08$ & $0.92 \pm 0.04$ & $0.92 \pm 0.03$ & $0.84 \pm 0.05$ \\
\hline tempdiag & $1.00 \pm 0.00$ & $1.00 \pm 0.00$ & $1.00 \pm 0.00$ & $1.00 \pm 0.00$ \\
\hline tep.fea & $0.65 \pm 0.00$ & $0.65 \pm 0.02$ & $0.65 \pm 0.02$ & $0.65 \pm 0.02$ \\
\hline tic-tac-toe & $0.94 \pm 0.02$ & $0.94 \pm 0.02$ & $0.86 \pm 0.03$ & $0.86 \pm 0.03$ \\
\hline transfusion & $0.79 \pm 0.00$ & $0.79 \pm 0.03$ & $0.78 \pm 0.02$ & $0.78 \pm 0.02$ \\
\hline vehicle & $0.77 \pm 0.01$ & $0.72 \pm 0.04$ & $0.74 \pm 0.04$ & $0.71 \pm 0.04$ \\
\hline vote & $0.96 \pm 0.00$ & $0.97 \pm 0.02$ & $0.97 \pm 0.02$ & $0.95 \pm 0.03$ \\
\hline vowel & $0.76 \pm 0.07$ & $0.82 \pm 0.04$ & $0.83 \pm 0.03$ & $0.70 \pm 0.04$ \\
\hline wine-red & $0.64 \pm 0.01$ & $0.63 \pm 0.02$ & $0.61 \pm 0.03$ & $0.60 \pm 0.03$ \\
\hline wine-white & $0.55 \pm 0.01$ & $0.58 \pm 0.02$ & $0.61 \pm 0.03$ & $0.56 \pm 0.02$ \\
\hline Average Rank & 1.77 & 2.50 & 2.35 & 3.38 \\
\hline
\end{tabular}

(b) F-Measure results.

\begin{tabular}{lrrrr}
\hline \multicolumn{1}{c}{ Data Set } & HEAD & CART & C4.5 & REP \\
\hline abalone & $0.27 \pm 0.01$ & $0.23 \pm 0.02$ & $0.21 \pm 0.02$ & $0.24 \pm 0.02$ \\
anneal & $0.98 \pm 0.01$ & $0.98 \pm 0.01$ & $0.98 \pm 0.01$ & $0.98 \pm 0.02$ \\
arrhythmia & $0.76 \pm 0.02$ & $0.67 \pm 0.06$ & $0.65 \pm 0.06$ & $0.63 \pm 0.07$ \\
audiology & $0.77 \pm 0.01$ & $0.71 \pm 0.05$ & $0.75 \pm 0.08$ & $0.70 \pm 0.09$ \\
autos & $0.85 \pm 0.04$ & $0.77 \pm 0.10$ & $0.85 \pm 0.07$ & $0.62 \pm 0.07$ \\
breast-cancer & $0.73 \pm 0.00$ & $0.63 \pm 0.05$ & $0.70 \pm 0.11$ & $0.62 \pm 0.06$ \\
breast-w & $0.96 \pm 0.01$ & $0.95 \pm 0.02$ & $0.95 \pm 0.02$ & $0.94 \pm 0.03$ \\
bridges2 & $0.70 \pm 0.02$ & $0.43 \pm 0.05$ & $0.51 \pm 0.11$ & $0.29 \pm 0.11$ \\
car & $0.92 \pm 0.03$ & $0.97 \pm 0.02$ & $0.93 \pm 0.02$ & $0.89 \pm 0.02$ \\
heart-c & $0.83 \pm 0.01$ & $0.80 \pm 0.04$ & $0.76 \pm 0.09$ & $0.77 \pm 0.08$ \\
flags & $0.72 \pm 0.01$ & $0.57 \pm 0.10$ & $0.61 \pm 0.05$ & $0.58 \pm 0.10$ \\
credit-g & $0.76 \pm 0.01$ & $0.71 \pm 0.04$ & $0.70 \pm 0.02$ & $0.70 \pm 0.05$ \\
colic & $0.88 \pm 0.01$ & $0.84 \pm 0.08$ & $0.85 \pm 0.07$ & $0.83 \pm 0.07$ \\
heart-h & $0.83 \pm 0.01$ & $0.76 \pm 0.06$ & $0.80 \pm 0.07$ & $0.79 \pm 0.09$ \\
ionosphere & $0.94 \pm 0.01$ & $0.89 \pm 0.03$ & $0.88 \pm 0.05$ & $0.91 \pm 0.02$ \\
kdd-synthetic & $0.95 \pm 0.00$ & $0.88 \pm 0.04$ & $0.91 \pm 0.04$ & $0.87 \pm 0.04$ \\
kr-vs-kp & $0.96 \pm 0.00$ & $0.99 \pm 0.01$ & $0.99 \pm 0.01$ & $0.99 \pm 0.01$ \\
liver-disorders & $0.75 \pm 0.00$ & $0.66 \pm 0.09$ & $0.66 \pm 0.05$ & $0.63 \pm 0.05$ \\
lung-cancer & $0.71 \pm 0.02$ & $0.42 \pm 0.32$ & $0.35 \pm 0.29$ & $0.42 \pm 0.19$ \\
meta.data & $0.13 \pm 0.02$ & $0.02 \pm 0.01$ & $0.02 \pm 0.02$ & $0.00 \pm 0.00$ \\
morphological & $0.72 \pm 0.00$ & $0.70 \pm 0.04$ & $0.70 \pm 0.02$ & $0.70 \pm 0.03$ \\
mb-promoters & $0.89 \pm 0.01$ & $0.71 \pm 0.14$ & $0.79 \pm 0.14$ & $0.76 \pm 0.15$ \\
shuttle-control & $0.55 \pm 0.02$ & $0.57 \pm 0.39$ & $0.57 \pm 0.39$ & $0.57 \pm 0.39$ \\
soybean & $0.80 \pm 0.09$ & $0.91 \pm 0.04$ & $0.92 \pm 0.04$ & $0.82 \pm 0.06$ \\
tempdiag & $1.00 \pm 0.00$ & $1.00 \pm 0.00$ & $1.00 \pm 0.00$ & $1.00 \pm 0.00$ \\
tep.fea & $0.61 \pm 0.00$ & $0.61 \pm 0.02$ & $0.61 \pm 0.02$ & $0.61 \pm 0.02$ \\
tic-tac-toe & $0.94 \pm 0.02$ & $0.94 \pm 0.02$ & $0.86 \pm 0.03$ & $0.86 \pm 0.03$ \\
transfusion & $0.77 \pm 0.00$ & $0.76 \pm 0.03$ & $0.77 \pm 0.03$ & $0.76 \pm 0.02$ \\
vehicle & $0.77 \pm 0.01$ & $0.71 \pm 0.05$ & $0.73 \pm 0.04$ & $0.70 \pm 0.04$ \\
vote & $0.96 \pm 0.00$ & $0.97 \pm 0.02$ & $0.97 \pm 0.02$ & $0.95 \pm 0.03$ \\
vowel & $0.76 \pm 0.07$ & $0.82 \pm 0.04$ & $0.83 \pm 0.03$ & $0.70 \pm 0.04$ \\
wine-red & $0.63 \pm 0.01$ & $0.61 \pm 0.02$ & $0.61 \pm 0.03$ & $0.58 \pm 0.03$ \\
wine-white & $0.53 \pm 0.01$ & $0.58 \pm 0.03$ & $0.60 \pm 0.02$ & $0.55 \pm 0.02$ \\
\hline Average Rank & $\mathbf{1 . 7 1}$ & 2.53 & 2.29 & 3.47 \\
\hline & & & &
\end{tabular}

Table 6.21: Results for the $\{9 \times 31\}$ configuration.

(a) Accuracy results.

\begin{tabular}{lrrrr}
\hline \multicolumn{1}{c}{ Data Set } & HEAD & CART & C4.5 & REP \\
\hline abalone & $0.28 \pm 0.00$ & $0.26 \pm 0.02$ & $0.22 \pm 0.02$ & $0.26 \pm 0.02$ \\
anneal & $0.92 \pm 0.00$ & $0.98 \pm 0.01$ & $0.99 \pm 0.01$ & $0.98 \pm 0.02$ \\
arrhythmia & $0.76 \pm 0.00$ & $0.71 \pm 0.05$ & $0.66 \pm 0.05$ & $0.67 \pm 0.06$ \\
audiology & $0.67 \pm 0.01$ & $0.74 \pm 0.05$ & $0.78 \pm 0.07$ & $0.74 \pm 0.08$ \\
autos & $0.78 \pm 0.01$ & $0.78 \pm 0.10$ & $0.86 \pm 0.06$ & $0.65 \pm 0.08$ \\
breast-cancer & $0.73 \pm 0.00$ & $0.69 \pm 0.04$ & $0.75 \pm 0.08$ & $0.69 \pm 0.05$ \\
breast-w & $0.94 \pm 0.00$ & $0.95 \pm 0.02$ & $0.95 \pm 0.02$ & $0.94 \pm 0.03$ \\
bridges2 & $0.62 \pm 0.01$ & $0.54 \pm 0.08$ & $0.58 \pm 0.13$ & $0.40 \pm 0.15$ \\
car & $0.88 \pm 0.01$ & $0.97 \pm 0.02$ & $0.93 \pm 0.02$ & $0.89 \pm 0.02$ \\
heart-c & $0.82 \pm 0.00$ & $0.81 \pm 0.04$ & $0.77 \pm 0.09$ & $0.77 \pm 0.08$ \\
flags & $0.71 \pm 0.01$ & $0.61 \pm 0.10$ & $0.63 \pm 0.05$ & $0.62 \pm 0.10$ \\
credit-g & $0.75 \pm 0.00$ & $0.73 \pm 0.04$ & $0.71 \pm 0.03$ & $0.72 \pm 0.06$ \\
colic & $0.83 \pm 0.01$ & $0.85 \pm 0.08$ & $0.86 \pm 0.06$ & $0.83 \pm 0.06$ \\
heart-h & $0.80 \pm 0.00$ & $0.77 \pm 0.06$ & $0.80 \pm 0.08$ & $0.80 \pm 0.09$ \\
ionosphere & $0.92 \pm 0.00$ & $0.89 \pm 0.03$ & $0.89 \pm 0.05$ & $0.91 \pm 0.02$ \\
kdd--synthetic & $0.95 \pm 0.00$ & $0.88 \pm 0.04$ & $0.91 \pm 0.04$ & $0.88 \pm 0.03$ \\
kr-vs-kp & $0.91 \pm 0.01$ & $0.99 \pm 0.01$ & $0.99 \pm 0.01$ & $0.99 \pm 0.01$ \\
liver-disorders & $0.74 \pm 0.00$ & $0.67 \pm 0.09$ & $0.67 \pm 0.05$ & $0.65 \pm 0.05$ \\
meta.data & $0.11 \pm 0.00$ & $0.05 \pm 0.03$ & $0.04 \pm 0.03$ & $0.04 \pm 0.00$ \\
morphological & $0.71 \pm 0.00$ & $0.72 \pm 0.04$ & $0.72 \pm 0.02$ & $0.72 \pm 0.03$ \\
mb-promoters & $0.73 \pm 0.00$ & $0.72 \pm 0.14$ & $0.80 \pm 0.13$ & $0.77 \pm 0.15$ \\
shuttle-control & $0.63 \pm 0.00$ & $0.65 \pm 0.34$ & $0.65 \pm 0.34$ & $0.65 \pm 0.34$ \\
soybean & $0.64 \pm 0.01$ & $0.92 \pm 0.04$ & $0.92 \pm 0.03$ & $0.84 \pm 0.05$ \\
tep.fea & $0.65 \pm 0.00$ & $0.65 \pm 0.02$ & $0.65 \pm 0.02$ & $0.65 \pm 0.02$ \\
tic-tac-toe & $0.83 \pm 0.01$ & $0.94 \pm 0.02$ & $0.86 \pm 0.03$ & $0.86 \pm 0.03$ \\
transfusion & $0.79 \pm 0.00$ & $0.79 \pm 0.03$ & $0.78 \pm 0.02$ & $0.78 \pm 0.02$ \\
vehicle & $0.75 \pm 0.00$ & $0.72 \pm 0.04$ & $0.74 \pm 0.04$ & $0.71 \pm 0.04$ \\
vote & $0.96 \pm 0.00$ & $0.97 \pm 0.02$ & $0.97 \pm 0.02$ & $0.95 \pm 0.03$ \\
vowel & $0.61 \pm 0.01$ & $0.82 \pm 0.04$ & $0.83 \pm 0.03$ & $0.70 \pm 0.04$ \\
wine-red & $0.61 \pm 0.00$ & $0.63 \pm 0.02$ & $0.61 \pm 0.03$ & $0.60 \pm 0.03$ \\
wine-white & $0.53 \pm 0.00$ & $0.58 \pm 0.02$ & $0.61 \pm 0.03$ & $0.56 \pm 0.02$ \\
\hline Average Rank & 2.55 & 2.26 & $\mathbf{2 . 0 3}$ & 3.16 \\
\hline & & & &
\end{tabular}

(b) F-Measure results.

\begin{tabular}{lrrrr}
\hline \multicolumn{1}{c}{ Data Set } & HEAD & CART & C4.5 & REP \\
\hline abalone & $0.24 \pm 0.00$ & $0.23 \pm 0.02$ & $0.21 \pm 0.02$ & $0.24 \pm 0.02$ \\
anneal & $0.92 \pm 0.00$ & $0.98 \pm 0.01$ & $0.98 \pm 0.01$ & $0.98 \pm 0.02$ \\
arrhythmia & $0.73 \pm 0.00$ & $0.67 \pm 0.06$ & $0.65 \pm 0.06$ & $0.63 \pm 0.07$ \\
audiology & $0.62 \pm 0.01$ & $0.71 \pm 0.05$ & $0.75 \pm 0.08$ & $0.70 \pm 0.09$ \\
autos & $0.78 \pm 0.01$ & $0.77 \pm 0.10$ & $0.85 \pm 0.07$ & $0.62 \pm 0.07$ \\
breast-cancer & $0.71 \pm 0.00$ & $0.63 \pm 0.05$ & $0.70 \pm 0.11$ & $0.62 \pm 0.06$ \\
breast-w & $0.94 \pm 0.00$ & $0.95 \pm 0.02$ & $0.95 \pm 0.02$ & $0.94 \pm 0.03$ \\
bridges2 & $0.60 \pm 0.01$ & $0.43 \pm 0.05$ & $0.51 \pm 0.11$ & $0.29 \pm 0.11$ \\
car & $0.88 \pm 0.01$ & $0.97 \pm 0.02$ & $0.93 \pm 0.02$ & $0.89 \pm 0.02$ \\
heart-c & $0.82 \pm 0.00$ & $0.80 \pm 0.04$ & $0.76 \pm 0.09$ & $0.77 \pm 0.08$ \\
flags & $0.70 \pm 0.00$ & $0.57 \pm 0.10$ & $0.61 \pm 0.05$ & $0.58 \pm 0.10$ \\
credit-g & $0.73 \pm 0.00$ & $0.71 \pm 0.04$ & $0.70 \pm 0.02$ & $0.70 \pm 0.05$ \\
colic & $0.83 \pm 0.01$ & $0.84 \pm 0.08$ & $0.85 \pm 0.07$ & $0.83 \pm 0.07$ \\
heart-h & $0.79 \pm 0.00$ & $0.76 \pm 0.06$ & $0.80 \pm 0.07$ & $0.79 \pm 0.09$ \\
ionosphere & $0.92 \pm 0.00$ & $0.89 \pm 0.03$ & $0.88 \pm 0.05$ & $0.91 \pm 0.02$ \\
kdd-synthetic & $0.95 \pm 0.00$ & $0.88 \pm 0.04$ & $0.91 \pm 0.04$ & $0.87 \pm 0.04$ \\
kr-vs-kp & $0.91 \pm 0.01$ & $0.99 \pm 0.01$ & $0.99 \pm 0.01$ & $0.99 \pm 0.01$ \\
liver-disorders & $0.73 \pm 0.00$ & $0.66 \pm 0.09$ & $0.66 \pm 0.05$ & $0.63 \pm 0.05$ \\
meta.data & $0.09 \pm 0.00$ & $0.02 \pm 0.01$ & $0.02 \pm 0.02$ & $0.00 \pm 0.00$ \\
morphological & $0.69 \pm 0.00$ & $0.70 \pm 0.04$ & $0.70 \pm 0.02$ & $0.70 \pm 0.03$ \\
mb-promoters & $0.73 \pm 0.00$ & $0.71 \pm 0.14$ & $0.79 \pm 0.14$ & $0.76 \pm 0.15$ \\
shuttle-control & $0.56 \pm 0.00$ & $0.57 \pm 0.39$ & $0.57 \pm 0.39$ & $0.57 \pm 0.39$ \\
soybean & $0.60 \pm 0.02$ & $0.91 \pm 0.04$ & $0.92 \pm 0.04$ & $0.82 \pm 0.06$ \\
tep.fea & $0.61 \pm 0.00$ & $0.61 \pm 0.02$ & $0.61 \pm 0.02$ & $0.61 \pm 0.02$ \\
tic-tac-toe & $0.83 \pm 0.02$ & $0.94 \pm 0.02$ & $0.86 \pm 0.03$ & $0.86 \pm 0.03$ \\
transfusion & $0.77 \pm 0.00$ & $0.76 \pm 0.03$ & $0.77 \pm 0.03$ & $0.76 \pm 0.02$ \\
vehicle & $0.74 \pm 0.00$ & $0.71 \pm 0.05$ & $0.73 \pm 0.04$ & $0.70 \pm 0.04$ \\
vote & $0.96 \pm 0.00$ & $0.97 \pm 0.02$ & $0.97 \pm 0.02$ & $0.95 \pm 0.03$ \\
vowel & $0.60 \pm 0.01$ & $0.82 \pm 0.04$ & $0.83 \pm 0.03$ & $0.70 \pm 0.04$ \\
wine-red & $0.59 \pm 0.00$ & $0.61 \pm 0.02$ & $0.61 \pm 0.03$ & $0.58 \pm 0.03$ \\
wine-white & $0.49 \pm 0.00$ & $0.58 \pm 0.03$ & $0.60 \pm 0.02$ & $0.55 \pm 0.02$ \\
\hline Average Rank & 2.48 & 2.32 & $\mathbf{1 . 9 6}$ & 3.23 \\
\hline & & & &
\end{tabular}




\section{Discussion}

The experimental analysis conducted in the previous section aimed at comparing the performance of HEAD-DT when it is employed to generate an "all-around" decision-tree algorithm - i.e., an algorithm capable of performing reasonably well in a variety of distinct data sets. We once again measured the performance of each algorithm according to accuracy and F-Measure, which are the most well-known criteria for evaluating classification algorithms. In order to verify whether the number of data sets used in the meta-training set had an impact in the evolution of algorithms, we employed the same methodology as in the homogeneous approach, resulting in five different experimental configurations $\{$ \#meta-training sets, \#meta-test sets $\}:\{1 \times 39\},\{3 \times 37\}$, $\{5 \times 35\},\{7 \times 33\}$, and $\{9 \times 31\}$. By analysing the average rank obtained by each method in the previously mentioned configurations, we conclude that:

- C4.5 is the best-performing method, consistently presenting the lowest average rank values among the four algorithms employed in the experimental analysis;

- HEAD-DT is capable of generating competitive algorithms, and eventually the best-performing algorithm (e.g., configuration $\{7 \times 33\}$ ). Since its performance is heavily dependent on the data sets that comprise the meta-training set, it is to be expected that HEAD-DT eventually generates algorithms that are too-specific for the meta-training set (it suffers from a kind of "meta-overfitting"). Unfortunately, there is no easy solution to avoid this type of overfitting (see Section 6.2.3 for more details on this matter);

- REPTree is the worst-performing method in the group. This is expected given that its reduced-error pruning strategy is said to be effective only for very large data sets, considering that it requires a validation set.

For summarizing the average rank values obtained by each method in every experimental configuration, we gathered the rank values from Tables 6.17-6.21 in Table 6.22. Values in bold indicate the best performing method according to the corresponding evaluation measure.

The last step of this empirical analysis is to verify whether the differences in rank values are statistically significant. We employ once again the graphical representation suggested by Demšar (2006), the critical diagrams. Algorithms that are not significantly different from each other are connected. The critical difference given by the Nemenyi test is presented in the top of the graph.

Figure 6.3 shows the critical diagrams for all experimental configurations. It is interesting to see that there are no statistically significant differences among HEAD-DT, C4.5, and CART in neither configuration, except for $\{7 \times 33\}$, in which HEAD-DT outperforms CART with statistical significance (see Figure 6.3-(h)). Indeed, this is the only case in which one of the three algorithms outperform another in all five configurations. REPTree is often outperformed with statistical significance by the three other methods, which is expected considering it is consistently the worst-ranked algorithm among the four. 
Table 6.22: Summary of the experimental analysis regarding the heterogeneous approach.

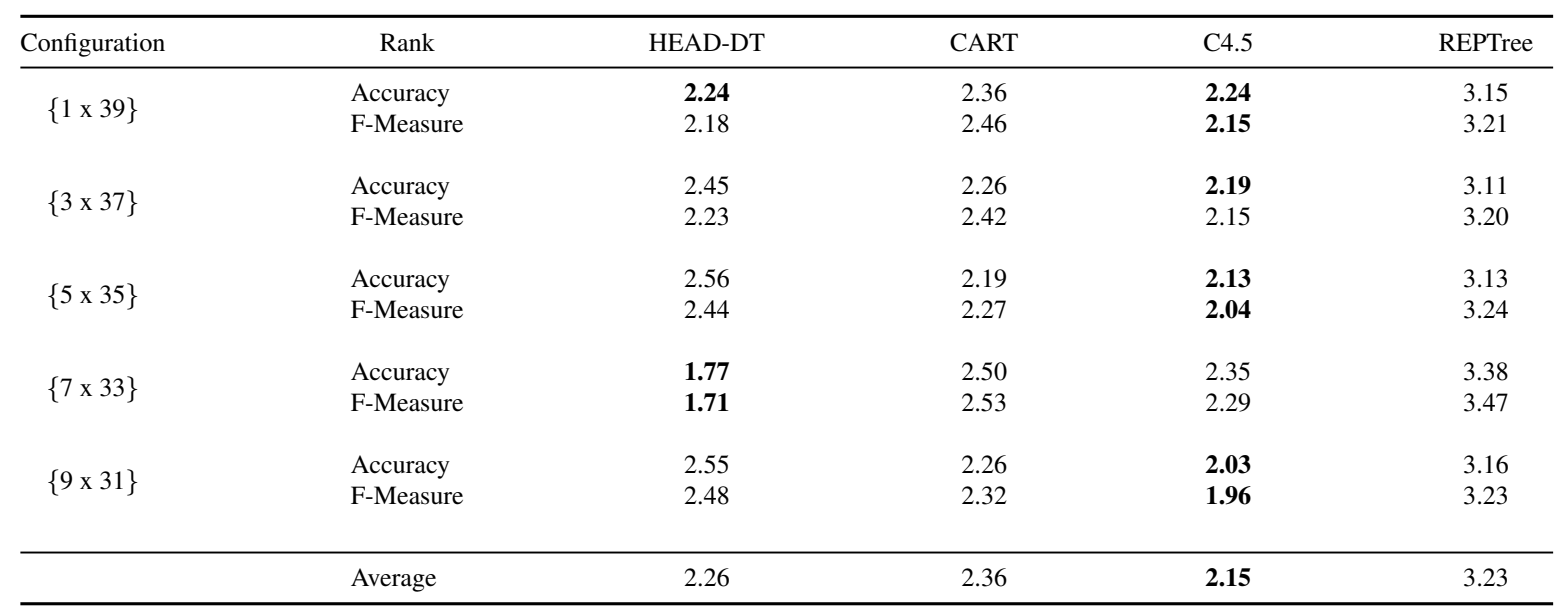

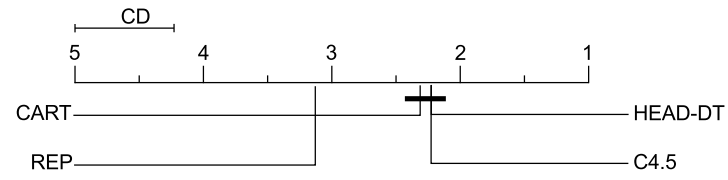

(a) Accuracy rank for $\{1 \times 39\}$.

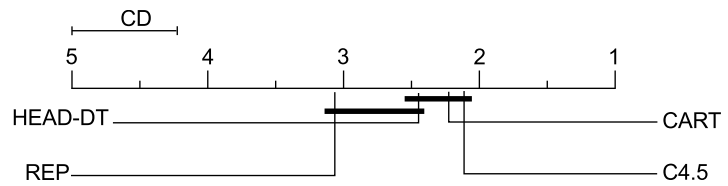

(c) Accuracy rank for $\{3 \times 37\}$.

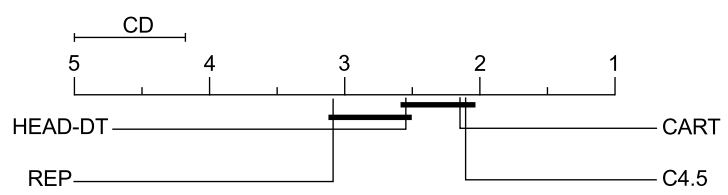

(e) Accuracy rank for $\{5 \times 35\}$.

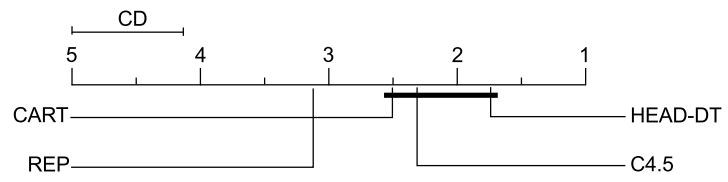

(g) Accuracy rank for $\{7 \times 33\}$.

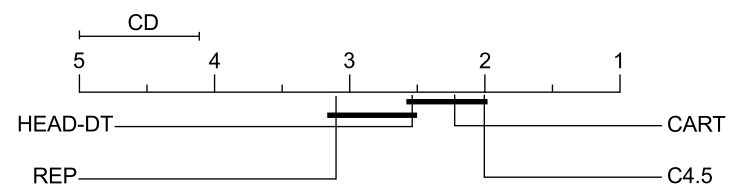

(i) Accuracy rank for $\{9 \times 31\}$.

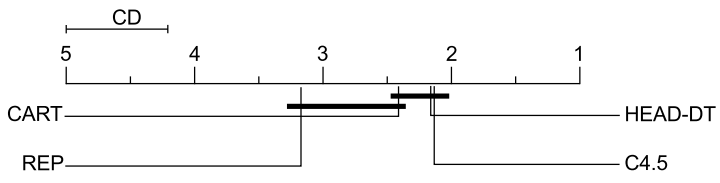

(b) F-Measure rank for $\{1 \times 39\}$.

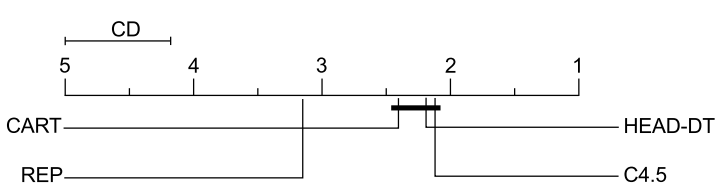

(d) F-Measure rank for $\{3 \times 37\}$.

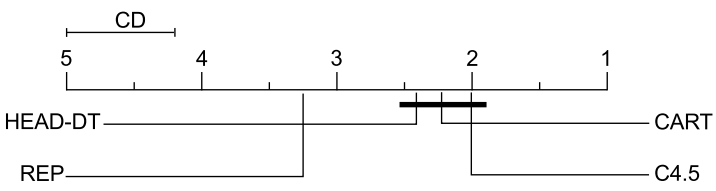

(f) F-Measure rank for $\{5 \times 35\}$.

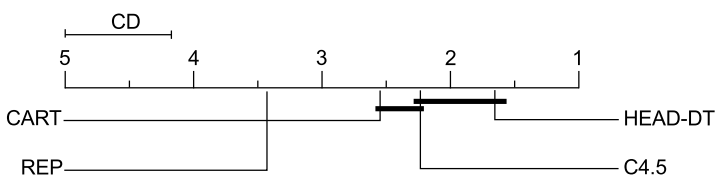

(h) F-Measure rank for $\{7 \times 33\}$.

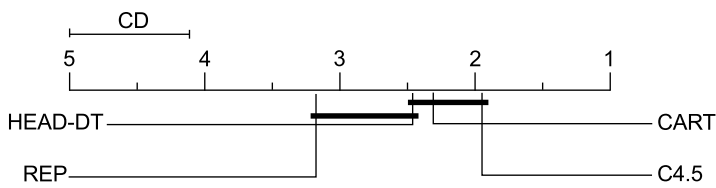

(j) F-Measure rank for $\{9 \times 31\}$.

Figure 6.3: Critical diagrams for the UCI data sets. 
Note that HEAD-DT did not achieve the same performance as in the homogeneous approach. That seems to show that generating an "all-around" algorithm is more difficult than generating a tailor-made algorithm for a particular domain. This is due to the own nature of the optimisation strategy employed by HEAD-DT. By optimising the building blocks of a decision-tree induction algorithm based on a few data sets in the meta-training set, HEAD-DT eventually finds a good compromise regarding the average F-Measure of these data sets. Nevertheless, we believe this automatically-designed algorithm to be too-specific to the meta-training data sets, leading to a specific case of overfitting. Our hypothesis is that HEAD-DT's generated algorithm is overfitting (at some extent) the meta-training set, causing some damage to its performance over the meta-test set. We further investigate this hypothesis in the next section, backing it up with data.

\subsubsection{The Case of Meta-Overfitting}

When evolving an algorithm from multiple data sets, HEAD-DT optimises its algorithms based on their predictive performance on a few data sets in the meta-training set. We saw in the previous section that generating an "all-around" algorithm that is capable of outperforming traditional algorithms, such as C4.5 and CART, is not an easy task. Our hypothesis for this apparent "lack of performance" is that HEAD-DT is finding a good (perhaps excellent) algorithm for the meta-training set, but that this algorithm is not really a good "all-around" algorithm. We call this phenomenon meta-overfitting ${ }^{4}$.

For supporting this hypothesis with data, let us analyse the performance of HEAD-DT in the meta-training set considering the previously-detailed configurations. Table 6.23 shows the F-Measure obtained by HEAD-DT in each data set in the meta-training set, as well as their average (fitness value). For a perspective view, we also present the same results for $\mathrm{C} 4.5$, considering the exact same data partition. Recall that, during the fitness evaluation process, we employ a random training-test partition for each data set, instead of typical 10-fold cross-validation procedure, in order to speed up evolution.

Table 6.23 shows that HEAD-DT is indeed designing algorithms that excel in the meta-training set. The average F-Measure achieved by HEAD-DT in the meta-training set is always greater than the one provided by C4.5. Whereas it is expected that HEAD-DT generates algorithms that perform well in the meta-training set (it is explicitly optimising these algorithms for that goal), the difference in performance between HEAD-DT and C4.5 is overwhelming. For instance, in configurations $\{3 \times 37\},\{5 \times 35\},\{7 \times 33\}$, and $\{9 \times 31\}$, HEAD-DT provides F-Measure values that are around $10 \%$ higher than those provided by C4.5. In configuration $\{1 \times 39\}$, in which HEAD-DT has to optimise a decision-tree induction algorithm based on the sole predictive performance of data set hayes-roth, the difference in F-Measure is even higher: $15 \%$ !

\footnotetext{
${ }^{4}$ The term overfitting is not used because it refers to a model that overfits the data, whereas we are talking about the case of an algorithm that "overfits" the data, in the sense that it is excellent when dealing with those data sets it was designed to, but it underperforms in previously unseen data sets.
} 
Table 6.23: F-Measure achieved by HEAD-DT and C4.5 in the meta-training set.

\begin{tabular}{|c|c|c|c|c|c|}
\hline Configuration & Data Sets & HEAD-DT & $\mathrm{C} 4.5$ & Average HEAD-DT & Average C4.5 \\
\hline$\left\{\begin{array}{lll}1 & \times & 39\end{array}\right\}$ & hayes-roth & 0.9242 & 0.7708 & 0.9242 & 0.7708 \\
\hline \multirow{3}{*}{$3 \times 37$} & hayes-roth & 0.9242 & 0.7708 & \multirow{3}{*}{0.8438} & \multirow{3}{*}{0.7352} \\
\hline & labor & 1.0000 & 0.8667 & & \\
\hline & tae & 0.6073 & 0.5682 & & \\
\hline \multirow{5}{*}{$5 \times 35$} & hayes-roth & 0.9242 & 0.7708 & \multirow{5}{*}{0.8494} & \multirow{5}{*}{0.7565} \\
\hline & labor & 1.0000 & 0.8667 & & \\
\hline & & 0.6073 & 0.5682 & & \\
\hline & & 0.9471 & 0.9471 & & \\
\hline & haberman & 0.7683 & 0.6298 & & \\
\hline \multirow{7}{*}{$7 \times 33$} & hayes-roth & 0.9242 & 0.7708 & \multirow{7}{*}{0.7808} & \multirow{7}{*}{0.6940} \\
\hline & labor & 0.8667 & 0.8667 & & \\
\hline & tae & 0.6017 & 0.5682 & & \\
\hline & iris & 0.9736 & 0.9471 & & \\
\hline & haberman & 0.7697 & 0.6298 & & \\
\hline & postoperative-patient & 0.6293 & 0.5708 & & \\
\hline & bridges 1 & 0.7003 & 0.5049 & & \\
\hline \multirow{9}{*}{$9 \times 31$} & hayes-roth & 0.8750 & 0.7708 & \multirow{9}{*}{0.7687} & \multirow{9}{*}{0.6628} \\
\hline & labor & 0.8038 & 0.8667 & & \\
\hline & tae & 0.6223 & 0.5682 & & \\
\hline & iris & 0.9471 & 0.9471 & & \\
\hline & haberman & 0.7342 & 0.6298 & & \\
\hline & postoperative-patient & 0.5708 & 0.5708 & & \\
\hline & bridges 1 & 0.6190 & 0.5049 & & \\
\hline & tempdiag & 1.0000 & 1.0000 & & \\
\hline & lung-cancer & 0.7464 & 0.1071 & & \\
\hline
\end{tabular}

These very good results achieved by HEAD-DT in the meta-training set and, at the same time, its disappointing results in the corresponding meta-test sets (except for configuration $\{7 \times 33\}$ ) seem to indicate that HEAD-DT is suffering from meta-overfitting. Unfortunately, the problem does not have a trivial solution. We comment on possible solutions to this problem in the thesis' future work possibilities, Section 8.2.

\subsection{HEAD-DT's Time Complexity}

Regarding execution time, it is clear that HEAD-DT is slower than C4.5, CART, or REPTree. Considering that there are 100 individuals executed for 100 generations, there is a maximum (worst case) of 10000 fitness evaluations of decision trees.

We recorded the execution time of both breeding operations and fitness evaluation (one thread was used for breeding and other for evaluation). Total time of breeding is negligible (a few milliseconds in a full evolutionary cycle), regardless of the data sets being used in the meta-training set (breeding does not consider any domain-specific information). Indeed, breeding individuals in the form of an integer string is known to be quite efficient in evolutionary computation.

Fitness evaluation, on the other hand, is the bottleneck of HEAD-DT. In the experiments of the specific framework, the largest data set (winequality_white) takes 2.5 hours to be fully executed (one full evolutionary cycle of 100 generations). The smallest data set (shuttle_landing) takes only 0.72 seconds to be fully executed. In the homogeneous approach of the general framework, the 
most time-consuming configuration, $\{9 \times 12\}$, takes 11.62 hours to be fully executed, whereas the fastest configuration, $\{1 \times 20\}$, takes only 5.60 minutes. Hence, we can see that the fitness evaluation time can vary quite a lot based on the number and type of data sets in the meta-training set.

The computational complexity of top-down decision-tree induction algorithms like $\mathrm{C} 4.5$ and CART is $O(m \times n \log n)$ ( $m$ is the number of attributes and $n$ the data set size), plus a term regarding the specific pruning method. Considering that breeding takes negligible time, we can say that in the worst case scenario, HEAD-DT time complexity is $O(i \times g \times m \times n \log n)$, where $i$ is the number of individuals and $g$ is the number of generations. In practice, the number of evaluations is much smaller than $i \times g$, due to the fact that repeated individuals are not re-evaluated. In addition, individuals selected by elitism and by reproduction (instead of crossover) are also not re-evaluated, saving computational time.

\subsection{Cost-Effectiveness of Automated vs Manual Algorithm Design}

The aforementioned conventional perspective for analysing HEAD-DT's time complexity is misleading in one way: it assumes that HEAD-DT is a "conventional" search algorithm, searching for an optimal solution to a single data set (as usual when running a classification algorithm), which is not the case. In reality, as discussed earlier, HEAD-DT is a hyper-heuristic that outputs a complete decision-tree induction algorithm. The algorithm automatically designed by HEAD-DT, as well as the manually-designed algorithms C4.5, CART and REPTree, are all complete decision-tree induction algorithms that can be re-used over and over again to extract knowledge from different data sets. In the machine learning literature, the time taken by human researchers to manually design an algorithm is usually not reported, but it is safe to assume that the time taken by a single human researcher to design and implement a new decision-tree induction algorithm is on the order of at least several months. In this context, noting that what HEAD-DT is doing is effectively replacing the manual design of decision-tree algorithms with an automated approach for such a design, even if HEAD-DT took a couple of days to produce a decision-tree algorithm, that time would still be much smaller than the corresponding manual design time. Hence, when evaluated as an algorithm-design method (which is really the role of HEAD-DT), it is fair to say it is a very fast method, at least much faster than a manual approach for the design of decision-tree induction algorithms.

At this point in our discussion, trying to play the role of devil's lawyer, one could perhaps present the following counter-argument: the aforementioned discussion is ignoring the fact that HEAD-DT was itself designed by human researchers, a design process that also took several months! This is of course true, but even taking this into account, it can be argued that HEAD-DT is still much more cost-effective than the human design of decision-tree algorithms, as follows. 
First, now that HEAD-DT has been manually designed, it can be re-used over and over again to automatically create decision-tree algorithms tailored to any particular type of data set (or application domain) in which a given user is interested. In the general framework, we focused on gene expression data sets, but HEAD-DT can be re-used to create decision-tree algorithms tailored to, say, a specific type of financial data sets or a specific type of medical data sets, to mention just two out of a very large number of possible application domains. Once HEAD-DT has been created, the "cost" associated with using HEAD-DT in any other application domain is very small - it is essentially the cost associated with the time to run HEAD-DT in a new application domain (say a couple of days of a desktop computer's processing time).

In contrast, what would be the cost of manually creating a new decision-tree algorithm tailored to a particular type of data set or application domain? First, note that this kind of manual design of a decision-tree algorithm tailored to a specific type of data set is hardly found in the literature. This is presumably because, for the new algorithm to be effective and really tailored to the target type of data set, a human researcher would need to be an expert in both decision-tree algorithms and the application domain (or more specifically the type of data set to be mined), and not many researchers would satisfy both criteria in practice. Just for the sake of argument, though, let us make the (probably unrealistic) assumption that there are many application domains for which there is a researcher who is an expert in both that application domain and decision-tree induction algorithms. For each such application domain, it seems safe to assume again that the human expert in question would need on the order of several months to design a new decision-tree algorithm that is effective and really tailored to that application domain; whilst, as mentioned earlier, HEAD-DT could automatically design this algorithm in a couple of days.

In summary, given the very large diversity of application domains to which decision-tree algorithms have been and will probably continue to be applied for a long time, one can see that HEAD-DT's automated approach offers a much more cost-effective approach for designing decision-tree algorithms than the conventional manual design approach that is nearly always used in machine learning research. In this sense, HEAD-DT paves the way for the large-scale and cost-effective production of decision-tree induction algorithms that are tailored to any specific application domain or type of classification data set of interest.

\subsection{Examples of Automatically-Designed Algorithms}

For illustrating a novel algorithm designed by HEAD-DT, let us first consider the specific framework, more specifically the algorithm designed to the Semeion data set, in which HEAD-DT managed to achieve maximum accuracy and F-Measure (which was not the case of CART and C4.5). The algorithm designed by HEAD-DT is presented in Algorithm 7. It is indeed novel, since no algorithm in the literature combines components such as the Chandra-Varghese criterion with a pruning-free strategy. 


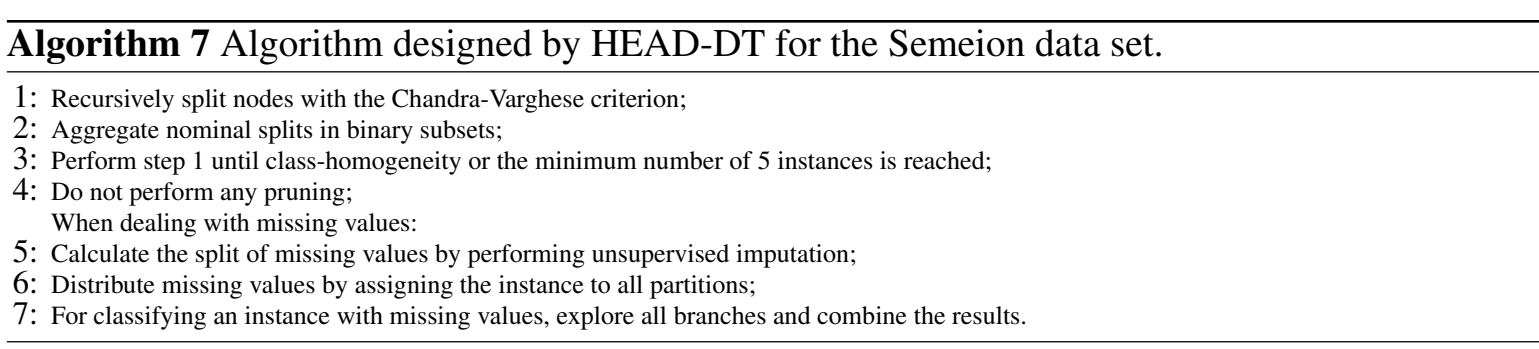

The main advantage of HEAD-DT is that it automatically searches for the suitable components (with their own biases) according to the data set (or set of data sets) being investigated. It is difficult to believe that a researcher would combine such a distinct set of components like those in Algorithm 7 to achieve 100\% accuracy in a particular data set.

Now let us consider the general framework, more specifically the homogeneous approach, in which HEAD-DT managed to outperform C4.5, CART, and REPTree for both accuracy and F-Measure. The typical algorithm designed by HEAD-DT for the domain of gene expression classification is presented in Algorithm 8. It is indeed novel, since no algorithm in the literature combines components such as the DCSM criterion with PEP pruning.

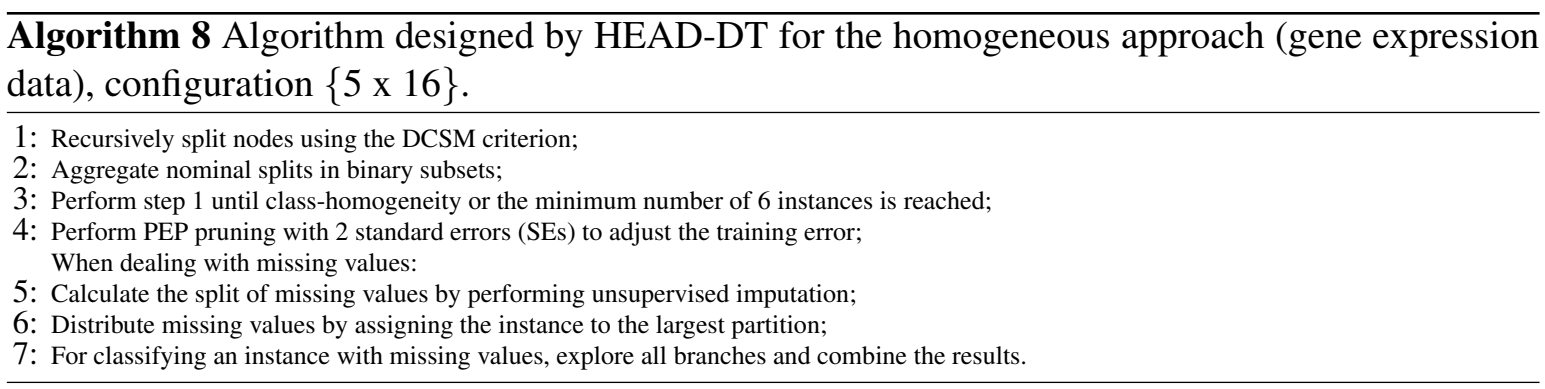

The algorithm presented in Algorithm 8 is one of the twenty-five algorithms automatically designed by HEAD-DT in the experimental analysis ( 5 configurations executed 5 times each). Nevertheless, by close inspection of the 25 automatically-generated algorithms, we observed that Algorithm 8 comprises building blocks that were consistently favored regarding the gene expression application domain. For instance, the DCSM and Chandra-Varghese criteria - both created recently by the same authors (Chandra et al., 2010; Chandra and Varghese, 2009) - were selected as the best split criterion in $\approx 50 \%$ of the algorithms designed by HEAD-DT. Similarly, the minimum number of instances stop criterion was selected in $\approx 70 \%$ of the algorithms, with either 6 or 7 instances as its parameter value. Finally, the PEP pruning was the favored pruning strategy in $\approx 60 \%$ of the algorithms, with a very large advantage over the strategies used by $\mathrm{C} 4.5$ (EBP pruning, selected in $8 \%$ of the algorithms) and CART (CCP pruning, not selected by any of the automatically-designed algorithms). 


\subsection{Is the Genetic Search Worthwhile?}

Finally, the last task to be performed in this chapter is to verify whether the genetic search employed by HEAD-DT provides solutions statistically better than a random walk through the search space. For that, we implemented the random search algorithm depicted in Algorithm 9.

The random search algorithm searches in the space of 10000 individuals in order to make a fair comparison with an evolutionary algorithm that evolves 100 individuals in 100 generations. These 10000 individuals are randomly created by generating random values from a uniform distribution to each of the individual's genes, within its valid boundaries (Algorithm 9, line 5).

After the genome is decoded in the form of a decision-tree induction algorithm (Algorithm 9, line 7), the randomly-generated algorithm is executed over the meta-training set (Algorithm 9, lines 8-11), and its fitness is computed as the average F-Measure achieved in each data set from the meta-training set (Algorithm 9, line 12). The best individual is stored in the variable bestAlgorithm (Algorithm 9, lines 13-16), and returned as the resulting decision-tree algorithm from the random search procedure.

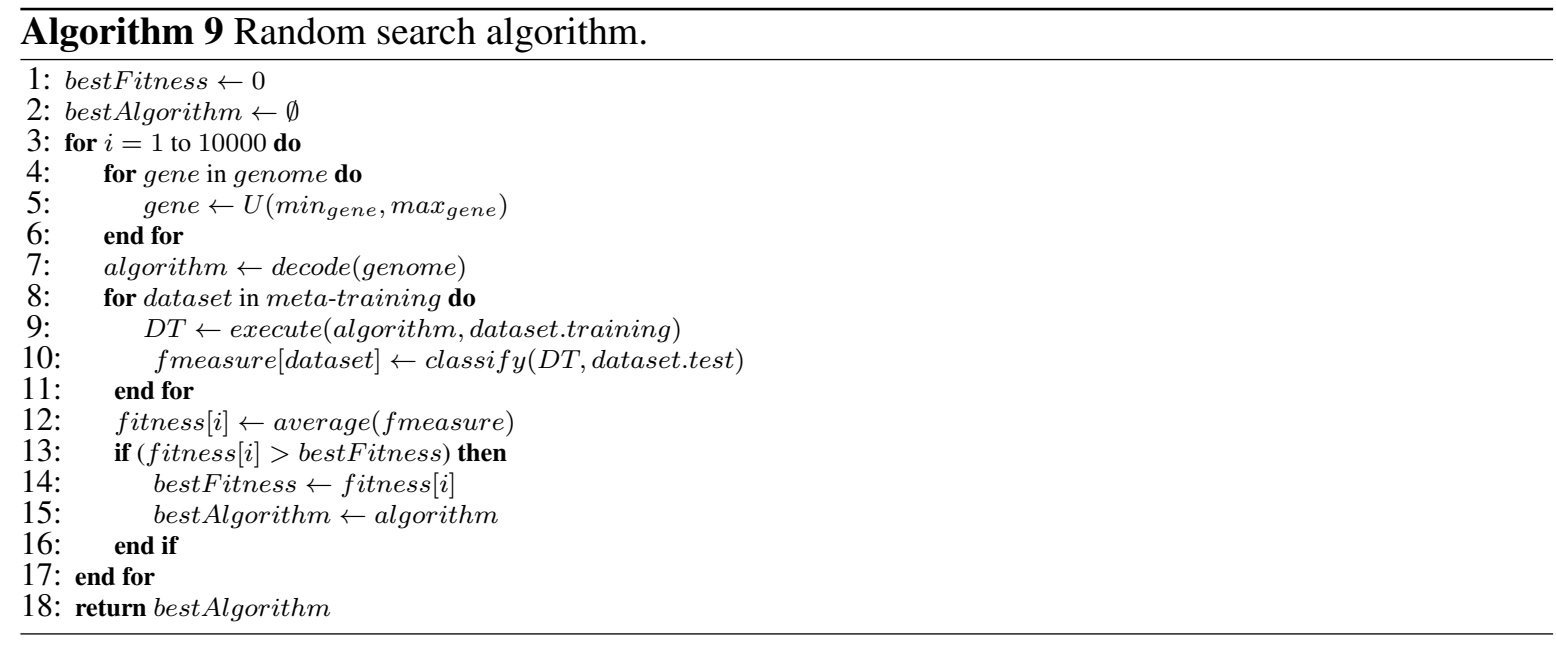

In order to compare the algorithms generated by HEAD-DT with those created by the random search algorithm, we employed the same experimental setup presented in Section 6.2.2: UCI data sets divided in 5 configurations: \{\#meta-training sets, \#meta-test sets $\}:\{1 \times 39\},\{3 \times 37\}$, $\{5 \times 35\},\{7 \times 33\}$, and $\{9 \times 31\}$. For evaluating the statistical significance of the results, we applied the Wilcoxon signed-ranks test (Wilcoxon, 1945), which is the recommended statistical test to evaluate two classifiers in multiple data sets (Demšar, 2006). In a nutshell, the Wilcoxon signed-ranks test is a non-parametric alternative to the well-known paired t-test, which ranks the differences in performances of two classifiers for each data set (ignoring their signs) and compares the ranks for the positive and negative differences. The sum of the positive and negative ranks are performed, and the smaller of them is compared to a table of exact critical values (for up to 25 data sets), and for larger values a $z$ statistic is computed and assumed to be normally distributed. 
Tables $6.24-6.28$ present the comparison results from the 5 configurations, for both accuracy and F-Measure. At the bottom of each table, we present the number of victories for each method (ties are omitted), and also the $p$-value returned by the Wilcoxon test. For rejecting the null hypothesis of performance equivalency between the two algorithms, the $p$-values should be smaller than the desired significance level $\alpha$. Note that, regardless of the configuration, the $p$-values are really small, showing that HEAD-DT is significantly better than the random search algorithm considering $\alpha=0.05$ and also $\alpha=0.01$. These results support the hypothesis that the genetic search employed by HEAD-DT is indeed worthwhile, allowing an effective search in the space of decision-tree induction algorithms.

Table 6.24: HEAD-DT vs random search: $\{1 \times 39\}$ configuration.

(a) Accuracy results.

\begin{tabular}{|c|c|c|}
\hline Data Set & HEAD-DT & Random Search \\
\hline abalone & $0.36 \pm 0.10$ & $0.25 \pm 0.01$ \\
\hline anneal & $0.92 \pm 0.05$ & $0.93 \pm 0.10$ \\
\hline arrhythmia & $0.66 \pm 0.08$ & $0.58 \pm 0.04$ \\
\hline audiology & $0.70 \pm 0.06$ & $0.56 \pm 0.19$ \\
\hline autos & $0.80 \pm 0.01$ & $0.64 \pm 0.16$ \\
\hline breast-cancer & $0.73 \pm 0.02$ & $0.68 \pm 0.04$ \\
\hline breast-w & $0.94 \pm 0.02$ & $0.94 \pm 0.01$ \\
\hline bridges 1 & $0.70 \pm 0.03$ & $0.56 \pm 0.11$ \\
\hline bridges2 & $0.64 \pm 0.07$ & $0.56 \pm 0.11$ \\
\hline car & $0.86 \pm 0.07$ & $0.88 \pm 0.04$ \\
\hline heart-c & $0.83 \pm 0.02$ & $0.76 \pm 0.03$ \\
\hline flags & $0.71 \pm 0.04$ & $0.56 \pm 0.15$ \\
\hline credit-g & $0.78 \pm 0.03$ & $0.72 \pm 0.01$ \\
\hline colic & $0.74 \pm 0.07$ & $0.70 \pm 0.10$ \\
\hline haberman & $0.77 \pm 0.01$ & $0.72 \pm 0.02$ \\
\hline heart-h & $0.83 \pm 0.02$ & $0.76 \pm 0.07$ \\
\hline ionosphere & $0.90 \pm 0.03$ & $0.89 \pm 0.02$ \\
\hline iris & $0.96 \pm 0.01$ & $0.95 \pm 0.01$ \\
\hline kdd-synthetic & $0.93 \pm 0.03$ & $0.88 \pm 0.03$ \\
\hline kr-vs-kp & $0.91 \pm 0.06$ & $0.87 \pm 0.20$ \\
\hline labor & $0.79 \pm 0.06$ & $0.69 \pm 0.04$ \\
\hline liver-disorders & $0.77 \pm 0.04$ & $0.67 \pm 0.02$ \\
\hline lung-cancer & $0.66 \pm 0.03$ & $0.45 \pm 0.06$ \\
\hline meta.data & $0.11 \pm 0.02$ & $0.03 \pm 0.01$ \\
\hline morphological & $0.73 \pm 0.03$ & $0.70 \pm 0.02$ \\
\hline mb-promoters & $0.78 \pm 0.07$ & $0.76 \pm 0.03$ \\
\hline postoperative-patient & $0.69 \pm 0.01$ & $0.67 \pm 0.05$ \\
\hline shuttle-control & $0.57 \pm 0.04$ & $0.51 \pm 0.10$ \\
\hline soybean & $0.69 \pm 0.20$ & $0.66 \pm 0.30$ \\
\hline tae & $0.64 \pm 0.03$ & $0.51 \pm 0.06$ \\
\hline tempdiag & $0.97 \pm 0.04$ & $0.97 \pm 0.05$ \\
\hline tep.fea & $0.65 \pm 0.00$ & $0.65 \pm 0.00$ \\
\hline tic-tac-toe & $0.83 \pm 0.08$ & $0.83 \pm 0.05$ \\
\hline transfusion & $0.80 \pm 0.01$ & $0.77 \pm 0.02$ \\
\hline vehicle & $0.78 \pm 0.04$ & $0.70 \pm 0.03$ \\
\hline vote & $0.95 \pm 0.00$ & $0.95 \pm 0.01$ \\
\hline vowel & $0.72 \pm 0.16$ & $0.63 \pm 0.09$ \\
\hline wine-red & $0.67 \pm 0.06$ & $0.57 \pm 0.01$ \\
\hline wine-white & $0.62 \pm 0.10$ & $0.53 \pm 0.02$ \\
\hline Number of victories: & 34 & 4 \\
\hline Wilcoxon $p$-value: & \multicolumn{2}{|c|}{$1.62 \times 10^{-9}$} \\
\hline
\end{tabular}

(b) F-Measure results.

\begin{tabular}{lcc}
\hline \multicolumn{1}{c}{ Data Set } & HEAD-DT & Random Search \\
\hline abalone & $0.34 \pm 0.11$ & $0.77 \pm 0.01$ \\
anneal & $0.90 \pm 0.08$ & $0.09 \pm 0.14$ \\
arrhythmia & $0.60 \pm 0.11$ & $0.53 \pm 0.10$ \\
audiology & $0.66 \pm 0.06$ & $0.50 \pm 0.22$ \\
autos & $0.80 \pm 0.01$ & $0.39 \pm 0.19$ \\
breast-cancer & $0.71 \pm 0.03$ & $0.36 \pm 0.05$ \\
breast-w & $0.94 \pm 0.02$ & $0.06 \pm 0.01$ \\
bridges1 & $0.68 \pm 0.04$ & $0.50 \pm 0.13$ \\
bridges2 & $0.62 \pm 0.08$ & $0.51 \pm 0.12$ \\
car & $0.85 \pm 0.08$ & $0.12 \pm 0.04$ \\
heart-c & $0.83 \pm 0.02$ & $0.24 \pm 0.04$ \\
flags & $0.70 \pm 0.05$ & $0.48 \pm 0.21$ \\
credit-g & $0.77 \pm 0.04$ & $0.32 \pm 0.06$ \\
colic & $0.72 \pm 0.09$ & $0.37 \pm 0.15$ \\
haberman & $0.75 \pm 0.01$ & $0.31 \pm 0.02$ \\
heart-h & $0.83 \pm 0.02$ & $0.27 \pm 0.11$ \\
ionosphere & $0.90 \pm 0.04$ & $0.12 \pm 0.02$ \\
iris & $0.96 \pm 0.01$ & $0.05 \pm 0.01$ \\
kdd-synthetic & $0.93 \pm 0.03$ & $0.12 \pm 0.03$ \\
kr-vs-kp & $0.91 \pm 0.06$ & $0.16 \pm 0.27$ \\
labor & $0.76 \pm 0.09$ & $0.38 \pm 0.05$ \\
liver-disorders & $0.77 \pm 0.04$ & $0.33 \pm 0.02$ \\
lung-cancer & $0.65 \pm 0.04$ & $0.64 \pm 0.10$ \\
meta.data & $0.10 \pm 0.03$ & $0.98 \pm 0.01$ \\
morphological & $0.71 \pm 0.04$ & $0.31 \pm 0.01$ \\
mb-promoters & $0.78 \pm 0.07$ & $0.24 \pm 0.03$ \\
postoperative-patient & $0.64 \pm 0.02$ & $0.42 \pm 0.02$ \\
shuttle-control & $0.55 \pm 0.06$ & $0.60 \pm 0.05$ \\
soybean & $0.66 \pm 0.21$ & $0.37 \pm 0.33$ \\
tae & $0.64 \pm 0.03$ & $0.49 \pm 0.06$ \\
tempdiag & $0.97 \pm 0.04$ & $0.03 \pm 0.05$ \\
tep.fea & $0.61 \pm 0.00$ & $0.39 \pm 0.00$ \\
tic-tac-toe & $0.83 \pm 0.09$ & $0.18 \pm 0.05$ \\
transfusion & $0.78 \pm 0.01$ & $0.25 \pm 0.01$ \\
vehicle & $0.77 \pm 0.04$ & $0.30 \pm 0.03$ \\
vote & $0.95 \pm 0.00$ & $0.05 \pm 0.01$ \\
vowel & $0.71 \pm 0.17$ & $0.37 \pm 0.09$ \\
wine-red & $0.66 \pm 0.07$ & $0.44 \pm 0.02$ \\
wine-white & $0.60 \pm 0.12$ & $0.49 \pm 0.03$ \\
\hline Number of victories: & 36 & 3 \\
\hline & & \\
\hline & & \\
\hline
\end{tabular}


Table 6.25: HEAD-DT vs random search: $\{3 \times 37\}$ configuration.

(a) Accuracy results.

\begin{tabular}{lcc}
\hline \multicolumn{1}{c}{ Data Set } & HEAD-DT & Random Search \\
\hline abalone & $0.27 \pm 0.00$ & $0.25 \pm 0.01$ \\
anneal & $0.98 \pm 0.01$ & $0.93 \pm 0.10$ \\
arrhythmia & $0.66 \pm 0.10$ & $0.58 \pm 0.04$ \\
audiology & $0.73 \pm 0.02$ & $0.56 \pm 0.19$ \\
autos & $0.73 \pm 0.08$ & $0.64 \pm 0.16$ \\
breast-cancer & $0.74 \pm 0.01$ & $0.68 \pm 0.04$ \\
breast-w & $0.94 \pm 0.01$ & $0.94 \pm 0.01$ \\
bridges1 & $0.70 \pm 0.06$ & $0.56 \pm 0.11$ \\
bridges2 & $0.69 \pm 0.05$ & $0.56 \pm 0.11$ \\
car & $0.84 \pm 0.02$ & $0.88 \pm 0.04$ \\
heart-c & $0.81 \pm 0.00$ & $0.76 \pm 0.03$ \\
flags & $0.69 \pm 0.01$ & $0.56 \pm 0.15$ \\
credit-g & $0.74 \pm 0.00$ & $0.72 \pm 0.01$ \\
colic & $0.78 \pm 0.12$ & $0.70 \pm 0.10$ \\
haberman & $0.77 \pm 0.00$ & $0.72 \pm 0.02$ \\
heart-h & $0.80 \pm 0.01$ & $0.76 \pm 0.07$ \\
ionosphere & $0.92 \pm 0.03$ & $0.89 \pm 0.02$ \\
iris & $0.96 \pm 0.00$ & $0.95 \pm 0.01$ \\
kdd-synthetic & $0.95 \pm 0.01$ & $0.88 \pm 0.03$ \\
kr-vs-kp & $0.91 \pm 0.03$ & $0.87 \pm 0.20$ \\
liver-disorders & $0.73 \pm 0.01$ & $0.67 \pm 0.02$ \\
lung-cancer & $0.69 \pm 0.00$ & $0.45 \pm 0.06$ \\
meta.data & $0.08 \pm 0.04$ & $0.03 \pm 0.01$ \\
morphological & $0.71 \pm 0.00$ & $0.70 \pm 0.02$ \\
mb-promoters & $0.88 \pm 0.02$ & $0.76 \pm 0.03$ \\
postoperative-patient & $0.70 \pm 0.02$ & $0.67 \pm 0.05$ \\
shuttle-control & $0.60 \pm 0.02$ & $0.51 \pm 0.10$ \\
soybean & $0.79 \pm 0.06$ & $0.66 \pm 0.30$ \\
tempdiag & $1.00 \pm 0.00$ & $0.97 \pm 0.05$ \\
tep.fea & $0.65 \pm 0.00$ & $0.65 \pm 0.00$ \\
tic-tac-toe & $0.76 \pm 0.04$ & $0.83 \pm 0.05$ \\
transfusion & $0.79 \pm 0.01$ & $0.77 \pm 0.02$ \\
vehicle & $0.74 \pm 0.00$ & $0.70 \pm 0.03$ \\
vote & $0.95 \pm 0.01$ & $0.95 \pm 0.01$ \\
vowel & $0.59 \pm 0.08$ & $0.63 \pm 0.09$ \\
wine-red & $0.59 \pm 0.01$ & $0.57 \pm 0.01$ \\
wine-white & $0.52 \pm 0.01$ & $0.53 \pm 0.02$ \\
\hline Number of victories: & 30 & \\
$\quad$ Wilcoxon $p$-value: & $3.04 \times 10^{-6} 6$ \\
\hline & & \\
\hline
\end{tabular}

(b) F-Measure results.

\begin{tabular}{lcr}
\hline \multicolumn{1}{c}{ Data Set } & HEAD-DT & Random Search \\
\hline abalone & $0.23 \pm 0.00$ & $0.77 \pm 0.01$ \\
anneal & $0.97 \pm 0.01$ & $0.09 \pm 0.14$ \\
arrhythmia & $0.58 \pm 0.17$ & $0.53 \pm 0.10$ \\
audiology & $0.70 \pm 0.02$ & $0.50 \pm 0.22$ \\
autos & $0.73 \pm 0.08$ & $0.39 \pm 0.19$ \\
breast-cancer & $0.72 \pm 0.00$ & $0.36 \pm 0.05$ \\
breast-w & $0.94 \pm 0.01$ & $0.06 \pm 0.01$ \\
bridges1 & $0.68 \pm 0.06$ & $0.50 \pm 0.13$ \\
bridges2 & $0.68 \pm 0.06$ & $0.51 \pm 0.12$ \\
car & $0.83 \pm 0.02$ & $0.12 \pm 0.04$ \\
heart-c & $0.81 \pm 0.00$ & $0.24 \pm 0.04$ \\
flags & $0.68 \pm 0.01$ & $0.48 \pm 0.21$ \\
credit-g & $0.73 \pm 0.00$ & $0.32 \pm 0.06$ \\
colic & $0.72 \pm 0.18$ & $0.37 \pm 0.15$ \\
haberman & $0.75 \pm 0.01$ & $0.31 \pm 0.02$ \\
heart-h & $0.80 \pm 0.01$ & $0.27 \pm 0.11$ \\
ionosphere & $0.92 \pm 0.03$ & $0.12 \pm 0.02$ \\
iris & $0.96 \pm 0.00$ & $0.05 \pm 0.01$ \\
kdd-synthetic & $0.95 \pm 0.01$ & $0.12 \pm 0.03$ \\
kr-vs-kp & $0.91 \pm 0.03$ & $0.16 \pm 0.27$ \\
liver-disorders & $0.72 \pm 0.02$ & $0.33 \pm 0.02$ \\
lung-cancer & $0.69 \pm 0.00$ & $0.64 \pm 0.10$ \\
meta.data & $0.06 \pm 0.03$ & $0.98 \pm 0.01$ \\
morphological & $0.70 \pm 0.00$ & $0.31 \pm 0.01$ \\
mb-promoters & $0.88 \pm 0.02$ & $0.24 \pm 0.03$ \\
postoperative-patient & $0.67 \pm 0.02$ & $0.42 \pm 0.02$ \\
shuttle-control & $0.58 \pm 0.02$ & $0.60 \pm 0.05$ \\
soybean & $0.76 \pm 0.07$ & $0.37 \pm 0.33$ \\
tempdiag & $1.00 \pm 0.00$ & $0.03 \pm 0.05$ \\
tep.fea & $0.61 \pm 0.00$ & $0.39 \pm 0.00$ \\
tic-tac-toe & $0.76 \pm 0.04$ & $0.18 \pm 0.05$ \\
transfusion & $0.77 \pm 0.00$ & $0.25 \pm 0.01$ \\
vehicle & $0.74 \pm 0.00$ & $0.30 \pm 0.03$ \\
vote & $0.95 \pm 0.01$ & $0.05 \pm 0.01$ \\
vowel & $0.58 \pm 0.09$ & $0.37 \pm 0.09$ \\
wine-red & $0.57 \pm 0.01$ & $0.44 \pm 0.02$ \\
wine-white & $0.48 \pm 0.02$ & $0.49 \pm 0.03$ \\
\hline Number of victories: & 33 & 4 \\
Wilcoxon $p$-value: & & $8.27 \times 10^{-1}$ \\
\hline & & \\
\hline
\end{tabular}

Table 6.26: HEAD-DT vs random search: $\{5$ x 35$\}$ configuration.

(a) Accuracy results.

\begin{tabular}{lcc}
\hline \multicolumn{1}{c}{ Data Set } & HEAD-DT & Random Search \\
\hline abalone & $0.27 \pm 0.00$ & $0.25 \pm 0.01$ \\
anneal & $0.97 \pm 0.00$ & $0.93 \pm 0.10$ \\
arrhythmia & $0.58 \pm 0.08$ & $0.58 \pm 0.04$ \\
audiology & $0.76 \pm 0.00$ & $0.56 \pm 0.19$ \\
autos & $0.67 \pm 0.06$ & $0.64 \pm 0.16$ \\
breast-cancer & $0.75 \pm 0.00$ & $0.68 \pm 0.04$ \\
breast-w & $0.93 \pm 0.00$ & $0.94 \pm 0.01$ \\
bridges1 & $0.64 \pm 0.03$ & $0.56 \pm 0.11$ \\
bridges2 & $0.64 \pm 0.03$ & $0.56 \pm 0.11$ \\
car & $0.82 \pm 0.02$ & $0.88 \pm 0.04$ \\
heart-c & $0.81 \pm 0.01$ & $0.76 \pm 0.03$ \\
flags & $0.68 \pm 0.01$ & $0.56 \pm 0.15$ \\
credit-g & $0.75 \pm 0.00$ & $0.72 \pm 0.01$ \\
colic & $0.68 \pm 0.09$ & $0.70 \pm 0.10$ \\
heart-h & $0.77 \pm 0.05$ & $0.76 \pm 0.07$ \\
ionosphere & $0.89 \pm 0.00$ & $0.89 \pm 0.02$ \\
kdd-synthetic & $0.96 \pm 0.00$ & $0.88 \pm 0.03$ \\
kr-vs-kp & $0.95 \pm 0.00$ & $0.87 \pm 0.20$ \\
liver-disorders & $0.74 \pm 0.01$ & $0.67 \pm 0.02$ \\
lung-cancer & $0.69 \pm 0.00$ & $0.45 \pm 0.06$ \\
meta.data & $0.04 \pm 0.02$ & $0.03 \pm 0.01$ \\
morphological & $0.70 \pm 0.00$ & $0.70 \pm 0.02$ \\
mb-promoters & $0.86 \pm 0.01$ & $0.76 \pm 0.03$ \\
postoperative-patient & $0.72 \pm 0.02$ & $0.67 \pm 0.05$ \\
shuttle-control & $0.61 \pm 0.01$ & $0.51 \pm 0.10$ \\
soybean & $0.72 \pm 0.02$ & $0.66 \pm 0.30$ \\
tempdiag & $1.00 \pm 0.00$ & $0.97 \pm 0.05$ \\
tep.fea & $0.65 \pm 0.00$ & $0.65 \pm 0.00$ \\
tic-tac-toe & $0.73 \pm 0.03$ & $0.83 \pm 0.05$ \\
transfusion & $0.79 \pm 0.00$ & $0.77 \pm 0.02$ \\
vehicle & $0.74 \pm 0.00$ & $0.70 \pm 0.03$ \\
vote & $0.96 \pm 0.00$ & $0.95 \pm 0.01$ \\
vowel & $0.50 \pm 0.01$ & $0.63 \pm 0.09$ \\
wine-red & $0.60 \pm 0.00$ & $0.57 \pm 0.01$ \\
wine-white & $0.54 \pm 0.00$ & $0.53 \pm 0.02$ \\
\hline Number of victories: & 28 & 6 \\
$\quad$ Wilcoxon $p$-value: & $2.25 \times 10^{-4}$ \\
\hline & & \\
\hline
\end{tabular}

(b) F-Measure results.

\begin{tabular}{lcr}
\hline \multicolumn{1}{c}{ Data Set } & HEAD-DT & Random Search \\
\hline balone & $0.24 \pm 0.00$ & $0.77 \pm 0.01$ \\
anneal & $0.97 \pm 0.00$ & $0.09 \pm 0.14$ \\
arrhythmia & $0.45 \pm 0.13$ & $0.53 \pm 0.10$ \\
audiology & $0.73 \pm 0.00$ & $0.50 \pm 0.22$ \\
autos & $0.67 \pm 0.06$ & $0.39 \pm 0.19$ \\
breast-cancer & $0.73 \pm 0.00$ & $0.36 \pm 0.05$ \\
breast-w & $0.93 \pm 0.00$ & $0.06 \pm 0.01$ \\
bridges1 & $0.63 \pm 0.03$ & $0.50 \pm 0.13$ \\
bridges2 & $0.63 \pm 0.04$ & $0.51 \pm 0.12$ \\
car & $0.81 \pm 0.02$ & $0.12 \pm 0.04$ \\
heart-c & $0.80 \pm 0.01$ & $0.24 \pm 0.04$ \\
flags & $0.67 \pm 0.01$ & $0.48 \pm 0.21$ \\
credit-g & $0.73 \pm 0.00$ & $0.32 \pm 0.06$ \\
colic & $0.57 \pm 0.14$ & $0.37 \pm 0.15$ \\
heart-h & $0.74 \pm 0.07$ & $0.27 \pm 0.11$ \\
ionosphere & $0.89 \pm 0.01$ & $0.12 \pm 0.02$ \\
kdd-synthetic & $0.96 \pm 0.00$ & $0.12 \pm 0.03$ \\
kr-vs-kp & $0.95 \pm 0.00$ & $0.16 \pm 0.27$ \\
liver-disorders & $0.73 \pm 0.01$ & $0.33 \pm 0.02$ \\
lung-cancer & $0.69 \pm 0.00$ & $0.64 \pm 0.10$ \\
meta.data & $0.02 \pm 0.01$ & $0.98 \pm 0.01$ \\
morphological & $0.69 \pm 0.01$ & $0.31 \pm 0.01$ \\
mb-promoters & $0.86 \pm 0.01$ & $0.24 \pm 0.03$ \\
postoperative-patient & $0.69 \pm 0.03$ & $0.42 \pm 0.02$ \\
shuttle-control & $0.57 \pm 0.02$ & $0.60 \pm 0.05$ \\
soybean & $0.68 \pm 0.01$ & $0.37 \pm 0.33$ \\
tempdiag & $1.00 \pm 0.00$ & $0.03 \pm 0.05$ \\
tep.fea & $0.61 \pm 0.00$ & $0.39 \pm 0.00$ \\
tic-tac-toe & $0.72 \pm 0.04$ & $0.18 \pm 0.05$ \\
transfusion & $0.77 \pm 0.00$ & $0.25 \pm 0.01$ \\
vehicle & $0.74 \pm 0.00$ & $0.30 \pm 0.03$ \\
vote & $0.96 \pm 0.00$ & $0.05 \pm 0.01$ \\
vowel & $0.48 \pm 0.02$ & $0.37 \pm 0.09$ \\
wine-red & $0.59 \pm 0.00$ & $0.44 \pm 0.02$ \\
wine-white & $0.51 \pm 0.00$ & $0.49 \pm 0.03$ \\
\hline Number of victories: & 31 & 4 \\
Wilcoxon $p$-value: & & $7.84 \times 10-6$ \\
\hline & & \\
\hline
\end{tabular}


Table 6.27: HEAD-DT vs random search: $\{7$ x 33$\}$ configuration.

(a) Accuracy results.

\begin{tabular}{lcc}
\hline \multicolumn{1}{c}{ Data Set } & HEAD-DT & Random Search \\
\hline abalone & $0.29 \pm 0.01$ & $0.25 \pm 0.01$ \\
anneal & $0.98 \pm 0.01$ & $0.93 \pm 0.10$ \\
arrhythmia & $0.78 \pm 0.02$ & $0.58 \pm 0.04$ \\
audiology & $0.79 \pm 0.00$ & $0.56 \pm 0.19$ \\
autos & $0.84 \pm 0.04$ & $0.64 \pm 0.16$ \\
breast-cancer & $0.75 \pm 0.00$ & $0.68 \pm 0.04$ \\
breast-w & $0.96 \pm 0.01$ & $0.94 \pm 0.01$ \\
bridges2 & $0.71 \pm 0.01$ & $0.56 \pm 0.11$ \\
car & $0.92 \pm 0.02$ & $0.88 \pm 0.04$ \\
heart-c & $0.83 \pm 0.01$ & $0.76 \pm 0.03$ \\
flags & $0.73 \pm 0.01$ & $0.56 \pm 0.15$ \\
credit-g & $0.76 \pm 0.00$ & $0.72 \pm 0.01$ \\
colic & $0.88 \pm 0.01$ & $0.70 \pm 0.10$ \\
heart-h & $0.83 \pm 0.01$ & $0.76 \pm 0.07$ \\
ionosphere & $0.94 \pm 0.01$ & $0.89 \pm 0.02$ \\
kdd-synthetic & $0.95 \pm 0.00$ & $0.88 \pm 0.03$ \\
kr-vs-kp & $0.96 \pm 0.00$ & $0.87 \pm 0.20$ \\
liver-disorders & $0.75 \pm 0.00$ & $0.67 \pm 0.02$ \\
lung-cancer & $0.71 \pm 0.02$ & $0.45 \pm 0.06$ \\
meta.data & $0.14 \pm 0.02$ & $0.03 \pm 0.01$ \\
morphological & $0.74 \pm 0.01$ & $0.70 \pm 0.02$ \\
mb-promoters & $0.89 \pm 0.01$ & $0.76 \pm 0.03$ \\
shuttle-control & $0.59 \pm 0.02$ & $0.51 \pm 0.10$ \\
soybean & $0.82 \pm 0.08$ & $0.66 \pm 0.30$ \\
tempdiag & $1.00 \pm 0.00$ & $0.97 \pm 0.05$ \\
tep.fea & $0.65 \pm 0.00$ & $0.65 \pm 0.00$ \\
tic-tac-toe & $0.94 \pm 0.02$ & $0.83 \pm 0.05$ \\
transfusion & $0.79 \pm 0.00$ & $0.77 \pm 0.02$ \\
vehicle & $0.77 \pm 0.01$ & $0.70 \pm 0.03$ \\
vote & $0.96 \pm 0.00$ & $0.95 \pm 0.01$ \\
vowel & $0.76 \pm 0.07$ & $0.63 \pm 0.09$ \\
wine-red & $0.64 \pm 0.01$ & $0.57 \pm 0.01$ \\
wine-white & $0.55 \pm 0.01$ & $0.53 \pm 0.02$ \\
\hline Number of victories: & 32 & 0 \\
Wilcoxon $p$-value: & & $5.64 \times 10^{-}$ \\
\hline
\end{tabular}

(b) F-Measure results.

\begin{tabular}{lcc}
\hline \multicolumn{1}{c}{ Data Set } & HEAD-DT & Random Search \\
\hline abalone & $0.27 \pm 0.01$ & $0.77 \pm 0.01$ \\
anneal & $0.98 \pm 0.01$ & $0.09 \pm 0.14$ \\
arrhythmia & $0.76 \pm 0.02$ & $0.53 \pm 0.10$ \\
audiology & $0.77 \pm 0.01$ & $0.50 \pm 0.22$ \\
autos & $0.85 \pm 0.04$ & $0.39 \pm 0.19$ \\
breast-cancer & $0.73 \pm 0.00$ & $0.36 \pm 0.05$ \\
breast-w & $0.96 \pm 0.01$ & $0.06 \pm 0.01$ \\
bridges2 & $0.70 \pm 0.02$ & $0.51 \pm 0.12$ \\
car & $0.92 \pm 0.03$ & $0.12 \pm 0.04$ \\
heart-c & $0.83 \pm 0.01$ & $0.24 \pm 0.04$ \\
flags & $0.72 \pm 0.01$ & $0.48 \pm 0.21$ \\
credit-g & $0.76 \pm 0.01$ & $0.32 \pm 0.06$ \\
colic & $0.88 \pm 0.01$ & $0.37 \pm 0.15$ \\
heart-h & $0.83 \pm 0.01$ & $0.27 \pm 0.11$ \\
ionosphere & $0.94 \pm 0.01$ & $0.12 \pm 0.02$ \\
kdd-synthetic & $0.95 \pm 0.00$ & $0.12 \pm 0.03$ \\
kr-vs-kp & $0.96 \pm 0.00$ & $0.16 \pm 0.27$ \\
liver-disorders & $0.75 \pm 0.00$ & $0.33 \pm 0.02$ \\
lung-cancer & $0.71 \pm 0.02$ & $0.64 \pm 0.10$ \\
meta.data & $0.13 \pm 0.02$ & $0.98 \pm 0.01$ \\
morphological & $0.72 \pm 0.00$ & $0.31 \pm 0.01$ \\
mb-promoters & $0.89 \pm 0.01$ & $0.24 \pm 0.03$ \\
shuttle-control & $0.55 \pm 0.02$ & $0.60 \pm 0.05$ \\
soybean & $0.80 \pm 0.09$ & $0.37 \pm 0.33$ \\
tempdiag & $1.00 \pm 0.00$ & $0.03 \pm 0.05$ \\
tep.fea & $0.61 \pm 0.00$ & $0.39 \pm 0.00$ \\
tic-tac-toe & $0.94 \pm 0.02$ & $0.18 \pm 0.05$ \\
transfusion & $0.77 \pm 0.00$ & $0.25 \pm 0.01$ \\
vehicle & $0.77 \pm 0.01$ & $0.30 \pm 0.03$ \\
vote & $0.96 \pm 0.00$ & $0.05 \pm 0.01$ \\
vowel & $0.76 \pm 0.07$ & $0.37 \pm 0.09$ \\
wine-red & $0.63 \pm 0.01$ & $0.44 \pm \pm 0.02$ \\
wine-white & $0.53 \pm 0.01$ & $0.49 \pm 0.03$ \\
\hline Number of victories: & 30 & \\
$\quad$ Wilcoxon $p$-value: & & $6.36 \times 10$ \\
\hline & & \\
\hline & & \\
& & \\
\hline
\end{tabular}

Table 6.28: HEAD-DT vs random search: $\{9 \times 31\}$ configuration.

(a) Accuracy results.

\begin{tabular}{lcc}
\hline \multicolumn{1}{c}{ Data Set } & HEAD-DT & Random Search \\
\hline abalone & $0.28 \pm 0.00$ & $0.25 \pm 0.01$ \\
anneal & $0.92 \pm 0.00$ & $0.93 \pm 0.10$ \\
arrhythmia & $0.76 \pm 0.00$ & $0.58 \pm 0.04$ \\
audiology & $0.67 \pm 0.01$ & $0.56 \pm 0.19$ \\
autos & $0.78 \pm 0.01$ & $0.64 \pm 0.16$ \\
breast-cancer & $0.73 \pm 0.00$ & $0.68 \pm 0.04$ \\
breast-w & $0.94 \pm 0.00$ & $0.94 \pm 0.01$ \\
bridges2 & $0.62 \pm 0.01$ & $0.56 \pm 0.11$ \\
car & $0.88 \pm 0.01$ & $0.88 \pm 0.04$ \\
heart-c & $0.82 \pm 0.00$ & $0.76 \pm 0.03$ \\
flags & $0.71 \pm 0.01$ & $0.56 \pm 0.15$ \\
credit-g & $0.75 \pm 0.00$ & $0.72 \pm 0.01$ \\
colic & $0.83 \pm 0.01$ & $0.70 \pm 0.10$ \\
heart-h & $0.80 \pm 0.00$ & $0.76 \pm 0.07$ \\
ionosphere & $0.92 \pm 0.00$ & $0.89 \pm 0.02$ \\
kdd-synthetic & $0.95 \pm 0.00$ & $0.88 \pm 0.03$ \\
kr-vs-kp & $0.91 \pm 0.01$ & $0.87 \pm 0.20$ \\
liver-disorders & $0.74 \pm 0.00$ & $0.67 \pm 0.02$ \\
meta.data & $0.11 \pm 0.00$ & $0.03 \pm 0.01$ \\
morphological & $0.71 \pm 0.00$ & $0.70 \pm 0.02$ \\
mb-promoters & $0.73 \pm 0.00$ & $0.76 \pm 0.03$ \\
shuttle-control & $0.63 \pm 0.00$ & $0.51 \pm 0.10$ \\
soybean & $0.64 \pm 0.01$ & $0.66 \pm 0.30$ \\
tep.fea & $0.65 \pm 0.00$ & $0.65 \pm 0.00$ \\
tic-tac-toe & $0.83 \pm 0.01$ & $0.83 \pm 0.05$ \\
transfusion & $0.79 \pm 0.00$ & $0.77 \pm 0.02$ \\
vehicle & $0.75 \pm 0.00$ & $0.70 \pm 0.03$ \\
vote & $0.96 \pm 0.00$ & $0.95 \pm 0.01$ \\
vowel & $0.61 \pm 0.01$ & $0.63 \pm 0.09$ \\
wine-red & $0.61 \pm 0.00$ & $0.57 \pm 0.01$ \\
wine-white & $0.53 \pm 0.00$ & $0.53 \pm 0.02$ \\
\hline Number of victories: & 26 & \\
Wilcoxon $p$-value: & \multicolumn{2}{c}{$2.51 \times 10-5$} \\
\hline & & \\
\hline & &
\end{tabular}

(b) F-Measure results.

\begin{tabular}{lcc}
\hline \multicolumn{1}{c}{ Data Set } & HEAD-DT & Random Search \\
\hline abalone & $0.24 \pm 0.00$ & $0.77 \pm 0.01$ \\
anneal & $0.92 \pm 0.00$ & $0.09 \pm 0.14$ \\
arrhythmia & $0.73 \pm 0.00$ & $0.53 \pm 0.10$ \\
audiology & $0.62 \pm 0.01$ & $0.50 \pm 0.22$ \\
autos & $0.78 \pm 0.01$ & $0.39 \pm 0.19$ \\
breast-cancer & $0.71 \pm 0.00$ & $0.36 \pm 0.05$ \\
breast-w & $0.94 \pm 0.00$ & $0.06 \pm 0.01$ \\
bridges2 & $0.60 \pm 0.01$ & $0.51 \pm 0.12$ \\
car & $0.88 \pm 0.01$ & $0.12 \pm 0.04$ \\
heart-c & $0.82 \pm 0.00$ & $0.24 \pm 0.04$ \\
flags & $0.70 \pm 0.00$ & $0.48 \pm 0.21$ \\
credit-g & $0.73 \pm 0.00$ & $0.32 \pm 0.06$ \\
colic & $0.83 \pm 0.01$ & $0.37 \pm 0.15$ \\
heart-h & $0.79 \pm 0.00$ & $0.27 \pm 0.11$ \\
ionosphere & $0.92 \pm 0.00$ & $0.12 \pm 0.02$ \\
kdd-synthetic & $0.95 \pm 0.00$ & $0.12 \pm 0.03$ \\
kr-vs-kp & $0.91 \pm 0.01$ & $0.16 \pm 0.27$ \\
liver-disorders & $0.73 \pm 0.00$ & $0.33 \pm 0.02$ \\
meta.data & $0.09 \pm 0.00$ & $0.98 \pm 0.01$ \\
morphological & $0.69 \pm 0.00$ & $0.31 \pm 0.01$ \\
mb-promoters & $0.73 \pm 0.00$ & $0.24 \pm 0.03$ \\
shuttle-control & $0.56 \pm 0.00$ & $0.60 \pm 0.05$ \\
soybean & $0.60 \pm 0.02$ & $0.37 \pm 0.33$ \\
tep.fea & $0.61 \pm 0.00$ & $0.39 \pm 0.00$ \\
tic-tac-toe & $0.83 \pm 0.02$ & $0.18 \pm 0.05$ \\
transfusion & $0.77 \pm 0.00$ & $0.25 \pm 0.01$ \\
vehicle & $0.74 \pm 0.00$ & $0.30 \pm 0.03$ \\
vote & $0.96 \pm 0.00$ & $0.05 \pm 0.01$ \\
vowel & $0.60 \pm 0.01$ & $0.37 \pm 0.09$ \\
wine-red & $0.59 \pm 0.00$ & $0.44 \pm 0.02$ \\
wine-white & $0.49 \pm 0.00$ & $0.49 \pm 0.03$ \\
\hline Number of victories: & 28 & 3 \\
Wilcoxon $p$-value: & & $4.01 \times 10^{-5}$ \\
\hline & & \\
\hline
\end{tabular}




\subsection{Chapter Remarks}

In this chapter, we presented two distinct sets of experiments for assessing the effectiveness of HEAD-DT, according to the fitness evaluation frameworks presented in Chapter 5. In the first set of experiments, which concerned the specific framework, both the meta-training and meta-test sets comprise data belonging to a single data set. We evaluated the performance of algorithms automatically designed by HEAD-DT in 20 public UCI data sets, and we compared their performance with C4.5 and CART. Results showed that HEAD-DT is capable of generating specialized algorithms whose performance is significantly better than that of the baseline algorithms.

In the second set of experiments, which concerned the general framework, we evaluated HEAD-DT in two different scenarios: (i) the homogeneous approach, in which HEAD-DT evolved a single algorithm to be applied in data sets from a particular application domain, namely microarray gene expression data classification; and (ii) the heterogeneous approach, in which HEAD-DT evolved a single algorithm to be applied in a variety of data sets, aiming at generating an effective "all-around" algorithm.

In the homogeneous approach, HEAD-DT was the best-ranked method in all configurations, supporting the hypothesis that HEAD-DT is indeed capable of generating an algorithm tailor-made to a particular application domain. In the heterogeneous approach, HEAD-DT presented predictive performance similar to C4.5 and CART - no statistically-significant difference was found, except for a particular configuration in which HEAD-DT outperformed CART. By further analysing the data collected from the meta-training set, we concluded that HEAD-DT may be suffering from meta-overfitting, generating algorithms that excel in the meta-training set, but that underperform at previously-unseen data sets. Considering that CART and C4.5 algorithms are effective and efficient "all-around" algorithms, we recommend their use in those scenarios in which the user needs fast results in a broad and unrelated set of data.

We believe the sets of experiments presented in this chapter were comprehensive enough to conclude that HEAD-DT is an effective approach for building specialized decision-tree induction algorithms tailored to particular domains or individual data sets. We refer the interested reader to two successful domain-based applications of HEAD-DT: flexible-receptor molecular docking data (Barros et al., 2012b) and software maintenance effort prediction (Basgalupp et al., 2013).

We also presented in this chapter the time complexity of HEAD-DT, as well as two examples of automatically-designed decision-tree algorithms. Finally, we investigated whether the genetic search employed by HEAD-DT was really worthwhile by comparing it to a random-search strategy. Results clearly indicated that the evolutionary process effectively guides the search for robust decision-tree algorithms.

Even though HEAD-DT has already presented quite satisfactory results, we investigate in the next chapter several different strategies for using as HEAD-DT's fitness function in the general 
framework. We test both balanced and imbalanced data in the meta-training set used in the evolutionary process. 



$\overline{7}$

\section{HEAD-DT: Fitness Function Analysis}

In Chapter 5, more specifically in Section 5.4, we saw that the definition of a fitness function for the scenario in which HEAD-DT evolves a decision-tree algorithm from multiple data sets is an interesting and relevant problem. In the experiments presented in Chapter 6, Section 6.2, we employed a simple average over the F-Measure obtained in the data sets that belong to the meta-training set.

As previously observed, when evolving an algorithm from multiple data sets, each individual of HEAD-DT has to be executed over each data set in the meta-training set. Hence, instead of obtaining a single value of predictive performance, each individual scores a set of values that have to be eventually combined into a single measure. ${ }^{1}$

In this chapter, we analyse in more detail the impact of different strategies to be used as fitness function during the evolutionary cycle of HEAD-DT. We divide the experimental scheme into two distinct scenarios: (i) evolving a decision-tree induction algorithm from multiple balanced data sets; and (ii) evolving a decision-tree induction algorithm from multiple imbalanced data sets. In each of these scenarios, we analyse the difference in performance of well-known performance measures such as accuracy, F-Measure, AUC, recall, and also a lesser-known criterion, namely the relative accuracy improvement. In addition, we analyse different schemes of aggregation, such as simple average, median, and harmonic mean.

\footnotetext{
${ }^{1}$ Assuming we are not interested in dealing with a multi-objective optimisation problem.
} 


\subsection{Performance Measures}

Performance measures are key tools to assess the quality of machine learning approaches and models. Therefore, several different measures have been proposed in the specialized literature with the goal of providing better choices in general or for a specific application domain (Ferri et al., 2009).

In the context of HEAD-DT's fitness function, and given that it evaluates algorithms (individuals) over data sets, it is reasonable to assume that different classification performance measures could be employed to provide a quantitative assessment of algorithmic performance. In the next few sections, we present five different performance measures that were selected for further investigation as HEAD-DT's fitness function.

\subsubsection{Accuracy}

Probably the most well-known performance evaluation measure for classification problems, the accuracy of a model is the rate of correctly classified instances:

$$
\text { accuracy }=\frac{t p+t n}{t p+t n+f p+f n}
$$

where $t p(t n)$ stands for the true positives (true negatives) — instances correctly classified —, and $f p(f n)$ stands for the false positives (false negatives) — instances incorrectly classified.

Even though most classification algorithms are assessed regarding the accuracy they obtain in a data set, we must point out that accuracy may be a misleading performance measure. For instance, suppose we have a data set whose class distribution is very skewed: $90 \%$ of the instances belong to class A and $10 \%$ to class B. An algorithm that always classifies instances as belonging to class A would achieve $90 \%$ of accuracy, even though it never predicts a class-B instance. In this case, assuming that class B is equally important (or even more so) than class A, we would prefer an algorithm with lower accuracy, but which could eventually correctly predict some instances as belonging to the rare class $\mathrm{B}$.

\subsubsection{F-Measure}

As it was presented in Section 5.4, F-Measure (also F-score or $F_{1}$ score) is the harmonic mean of precision and recall:

$$
\begin{gathered}
\text { precision }=\frac{t p}{t p+f p} \\
\text { recall }=\frac{t p}{t p+f n}
\end{gathered}
$$




$$
f 1=2 \times \frac{\text { precision } \times \text { recall }}{\text { precision }+ \text { recall }}
$$

Note that though F-Measure is advocated in the machine learning literature as a single measure capable of capturing the effectiveness of a system, it still completely ignores the $t n$, which can vary freely without affecting the statistic (Powers, 2011).

\subsubsection{Area Under the ROC Curve}

The area under the ROC (receiver operating characteristic) curve (AUC) has been increasingly used as a performance evaluation measure in classification problems. The ROC curve graphically displays the trade-off between the true positive rate $(t p r=t p /(t p+f n))$ and the false positive rate $(f p r=f p /(f p+t n))$ of a classifier. ROC graphs have properties that make them especially useful for domains with skewed class distribution and unequal classification error costs (Fawcett, 2006).

To create the ROC curve, one needs to build a graph in which the tpr is plotted along the $y$ axis and the $f p r$ is shown on the $x$ axis. Each point along the curve corresponds to one of the models induced by a given algorithm, and different models are built by varying a probabilistic threshold that determines whether an instance should be classified as positive or negative. Figure 7.1 shows the ROC curves for a pair of classifiers, $M_{1}$ and $M_{2}$.

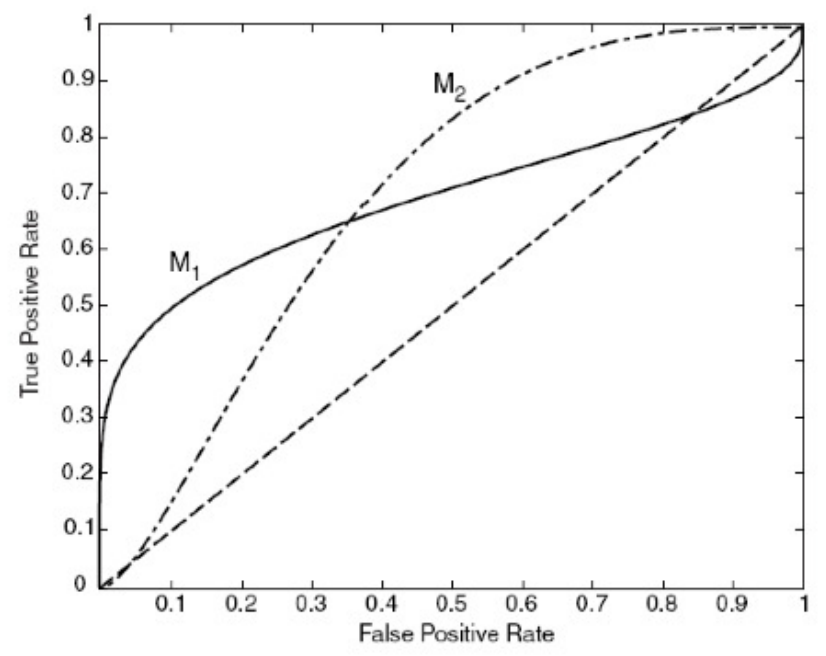

Figure 7.1: ROC curves for two different classifiers (Tan et al., 2005).

A ROC curve is a two-dimensional depiction of a classifier. To compare classifiers, we may want to reduce ROC performance to a single scalar value representing the expected performance, which is precisely the AUC. Since the AUC is a portion of the area of the unit square, its value will always be between 0 and 1 . However, because random guessing produces the diagonal dashed 
line between $(0,0)$ and $(1,1)$ (see Figure 7.1 ), which has an area of 0.5 , no realistic classifier should have an AUC value of less than 0.5. The AUC has an important statistical property: it is equivalent to the probability that the classifier will rank a randomly chosen positive instance higher than a randomly chosen negative instance, which makes of the AUC equivalent to the Wilcoxon test of ranks (Mason and Graham, 2002).

The machine learning community often uses the AUC statistic for model comparison, even though this practice has recently been questioned based upon new research that shows that AUC is quite noisy as a performance measure for classification (Hanczar et al., 2010) and has some other significant problems in model comparison (Hand, 2009; Lobo et al., 2008).

\subsubsection{Relative Accuracy Improvement}

Originally proposed by Pappa (2007), the relative accuracy improvement criterion measures the normalized improvement in accuracy of a given model over the data set's default accuracy (i.e., the accuracy achieved when using the majority class of the training data to classify the unseen data):

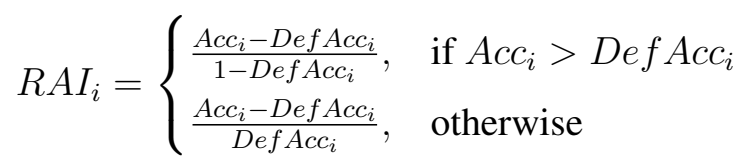

In Eq. (7.5), $A c c_{i}$ is the accuracy achieved by a given classifier in data set $i$, whereas $D e f A c c_{i}$ is the default accuracy of data set $i$. Note that if the improvement in accuracy is positive, i.e., the classifier accuracy is greater than the default accuracy, the improvement is normalized by the maximum possible improvement over the default accuracy $\left(1-D\right.$ ef $\left.A c c_{i}\right)$. Otherwise, the drop in the accuracy is normalized by the maximum possible drop, which is the value of the default accuracy itself. Hence, the relative accuracy improvement $R A I_{i}$ regarding data set $i$ returns a value between -1 (when $A c c_{i}=0$ ) and 1 (when $A c c_{i}=1$ ). Any improvement regarding the default accuracy results in a positive value, whereas any drop results in a negative value. In case $A c c_{i}=\operatorname{Def} A c c_{i}$ (i.e., no improvement or drop in accuracy is achieved), $R A I_{i}=0$, as expected.

The disadvantage of the relative accuracy improvement criterion is that it is not suitable for very imbalanced problems - data sets in which the default accuracy is really close to 1 -, since high accuracy does not properly translate into high performance for these kinds of problems, as we have previously seen.

\subsubsection{Recall}

Also known as sensitivity (usually in the medical field) or true positive rate, recall measures the proportion of actual positives that are correctly identified as such. For instance, it may refer to the percentage of sick patients who are correctly classified as having the particular disease. In terms of the confusion matrix terms, recall is computed as follows: 


$$
\text { recall }=\frac{t p}{t p+f n}
$$

Recall is useful for the case of imbalanced data, in which the positive class is the rare class. However, note that a classifier that always predicts the positive class will achieve a perfect recall, since recall does not take into consideration the $f p$ values. This problem is alleviated in multi-class problems, in which each class is used in turn as the positive class, and the average of the per-class recall is taken.

\subsection{Aggregation Schemes}

All classification measures presented in the previous section refer to the predictive performance of a given classifier in a given data set. When evolving an algorithm from multiple data sets, HEAD-DT's fitness function is measured as the aggregated performance of the individual in each data set that belongs to the meta-training set. We propose employing three simple strategies for combining the per-data-set performance into a single quantitative value: (i) simple average; (ii) median; and (iii) harmonic mean.

The simple average (or alternatively the arithmetic average) is computed by simply taking the average of the per-data-set values, i.e., $(1 / N) \times \sum_{i=1}^{N} p_{i}$, for a meta-training set with $N$ data sets and a performance measure $p$. It gives equal importance to the performance achieved in each data set. Moreover, it is best used in situations where there are no extreme outliers and the values are independent of each other.

The median is computed by ordering the performance values from smallest to greatest, and then taking the middle value of the ordered list. If there is an even number of data sets, since there is no single middle value, either $N / 2$ or $(N / 2)+1$ can be used as middle value, or alternatively their average. The median is robust to outliers in the data (extremely large or extremely low values that may influence the simple average).

Finally, the harmonic mean is given by $\left((1 / N) \times \sum_{i=1}^{N} p_{i}\right)^{-1}$. Unlike the simple average, the harmonic mean gives less significance to high-value outliers, providing sometimes a better picture of the average.

\subsection{Experimental Evaluation}

In this section, we perform an empirical evaluation of the five classification performance measures presented in Section 7.1 and the three aggregation schemes presented in Section 7.2 as fitness functions of HEAD-DT. There are a total of 15 distinct fitness functions resulting from this analysis:

1. Accuracy + Simple Average (ACC-A); 
2. Accuracy + Median $($ ACC-M);

3. Accuracy + Harmonic Mean $($ ACC-H $)$;

4. AUC + Simple Average (AUC-A);

5. AUC + Median (AUC-M);

6. AUC + Harmonic Mean (AUC-H);

7. F-Measure + Simple Average (FM-A);

8. F-Measure + Median (FM-M);

9. F-Measure + Harmonic Mean (FM-H);

10. Relative Accuracy Improvement + Simple Average (RAI-A);

11. Relative Accuracy Improvement + Median (RAI-M);

12. Relative Accuracy Improvement + Harmonic Mean (RAI-H);

13. Recall + Simple Average (TPR-A);

14. Recall + Median (TPR-M);

15. Recall + Harmonic Mean (TPR-H).

For this experiment, we employed the $67 \mathrm{UCI}$ data sets described in Table 6.14 organized into two scenarios: (i) 5 balanced data sets in the meta-training set; and (ii) 5 imbalanced data sets in the training set. These scenarios were created to assess the performance of the 15 distinct fitness functions in balanced and imbalanced data, considering that some of the performance measures are explicitly designed to deal with imbalanced data whereas others are not. The term "(im)balanced" was quantitatively measured according to the imbalance ratio (IR):

$$
I R=\frac{F\left(A_{D S}\right)}{F\left(B_{D S}\right)}
$$

where $F($.$) returns the frequency of a given class, A_{D S}$ is the highest-frequency class in data set $D S$ and $B_{D S}$ the lowest-frequency class in data set $D S$.

Given the size and complexity of this experiment, we did not optimise HEAD-DT's parameters as in Chapter 6, Section 6.2. Instead, we employed typical values found in the literature of evolutionary algorithms for decision-tree induction (the same parameters as in Chapter 6, Section 6.1):

- Population size: 100 ; 
- Maximum number of generations: 100;

- Selection: tournament selection with size $t=2$;

- Elitism rate: 5 individuals;

- Crossover: uniform crossover with $90 \%$ probability;

- Mutation: random uniform gene mutation with 5\% probability.

- Reproduction: cloning individuals with 5\% probability.

In the next sections, we present the results for both scenarios of meta-training set. Moreover, in the end of this chapter, we perform a whole new set of experiments with the best-performing fitness functions.

\subsubsection{Results for the Balanced Meta-Training Set}

We randomly selected 5 balanced data sets $(I R<1.1)$ from the $67 \mathrm{UCI}$ data sets described in Table 6.14 to be part of the meta-training set in this experiment: iris $(I R=1.0)$, segment $(I R=$ $1.0)$, vowel $(I R=1.0)$, mushroom $(I R=1.07)$, and kr-vs-kp $(I R=1.09)$.

Tables 7.1 and 7.2 show the results for the 62 data sets in the meta-test set regarding accuracy and F-Measure, respectively. At the bottom of each table, the average rank is presented for the 15 versions of HEAD-DT created by varying the fitness functions. We did not present standard deviation values due to space limitations within the tables.

By careful inspection of both tables, we can see that their rankings are practically the same, with the median of the relative accuracy improvement being the best-ranked method for either evaluation measure. Only a small position-switching occurs between the accuracy and F-Measure rankings, with respect to the positions of ACC-M, TPR-H, and FM-H.

Table 7.3 summarizes the average rank values obtained by each version of HEAD-DT with respect to accuracy and F-Measure. Values in bold indicate the best performing version according to the corresponding evaluation measure. It can be seen that version RAI-M is the best-performing method regardless of the evaluation measure. The average of the average ranks (average across evaluation measures) indicates the following final ranking positions (from best to worst): 1) RAI-M; 2) FM-M; 3) TPR-A; 4) RAI-A; 5) RAI-H; 6) TPR-M; 7) ACC-A; 8) FM-A; 9) ACC-H; 10) ACC-M; 11) FM-H; 12) TPR-H; 13) AUC-M; 14) AUC-A; 15) AUC-H.

For evaluating whether the differences between versions are statistically significant, we present the critical diagrams of the accuracy and F-Measure values in Figure 7.2. It is possible to observe that there are no significant differences among the top-4 versions (RAI-M, FM-M, TPR-A, and RAI-A). Nevertheless, RAI-M is the only version that outperforms TPR-M and RAI-H with 
Table 7.1: Accuracy values for the 15 versions of HEAD-DT varying the fitness functions. Meta-training comprises 5 balanced data sets.

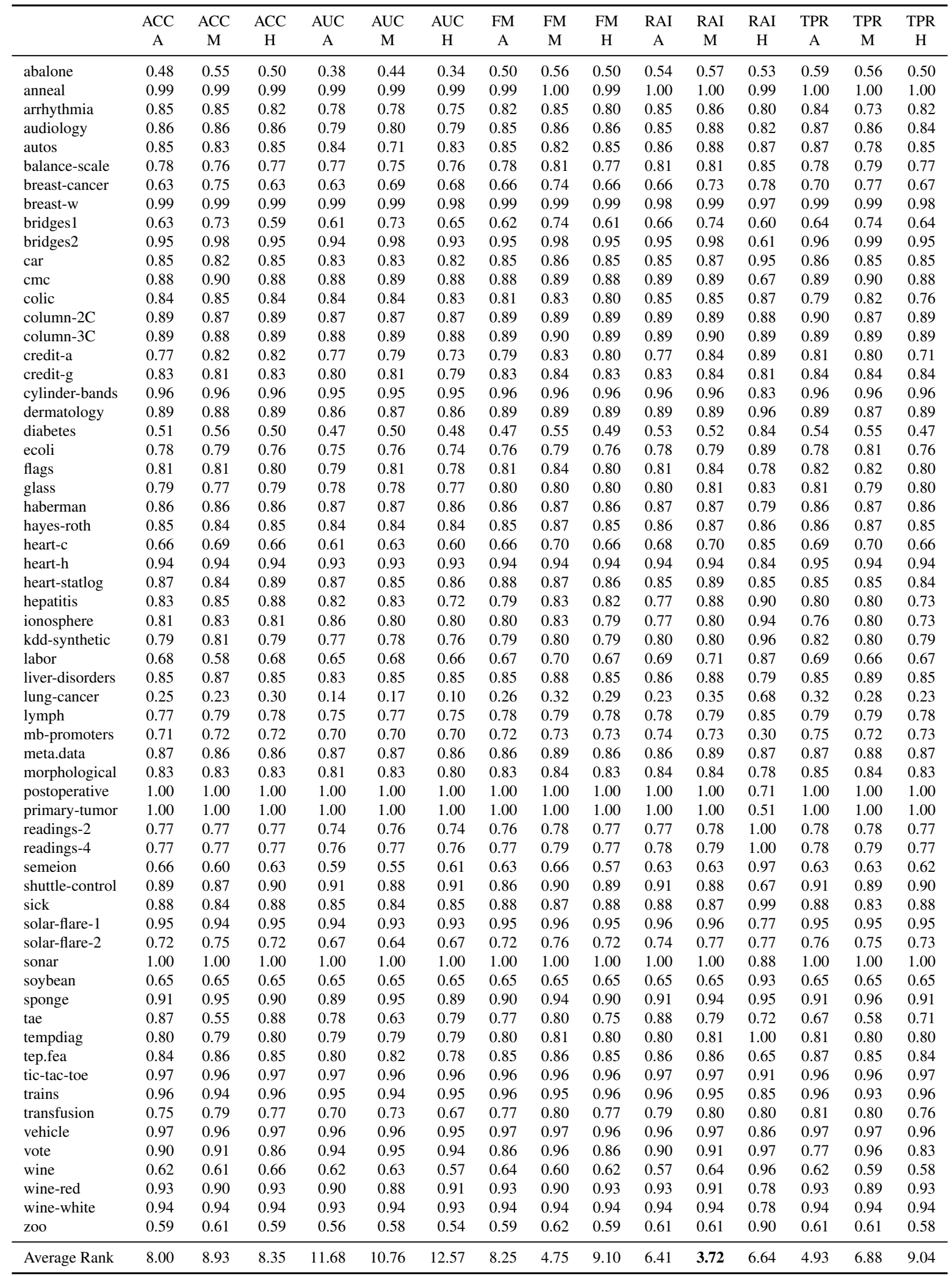


Table 7.2: F-Measure values for the 15 versions of HEAD-DT varying the fitness functions. Meta-training comprises 5 balanced data sets.

\begin{tabular}{|c|c|c|c|c|c|c|c|c|c|c|c|c|c|c|c|}
\hline & $\begin{array}{c}\mathrm{ACC} \\
\mathrm{A}\end{array}$ & $\begin{array}{c}\text { ACC } \\
\text { M }\end{array}$ & $\begin{array}{c}\text { ACC } \\
\mathrm{H}\end{array}$ & $\begin{array}{c}\text { AUC } \\
\text { A }\end{array}$ & $\begin{array}{c}\text { AUC } \\
M\end{array}$ & $\begin{array}{c}\text { AUC } \\
\mathrm{H}\end{array}$ & $\begin{array}{c}\text { FM } \\
\text { A }\end{array}$ & $\begin{array}{c}\text { FM } \\
\text { M }\end{array}$ & $\begin{array}{c}\text { FM } \\
\mathrm{H}\end{array}$ & $\begin{array}{c}\text { RAI } \\
\text { A }\end{array}$ & $\begin{array}{c}\text { RAI } \\
\text { M }\end{array}$ & $\begin{array}{c}\text { RAI } \\
\mathrm{H}\end{array}$ & $\begin{array}{c}\text { TPR } \\
\text { A }\end{array}$ & $\begin{array}{c}\text { TPR } \\
\mathrm{M}\end{array}$ & $\begin{array}{c}\text { TPR } \\
\mathrm{H}\end{array}$ \\
\hline abalone & 0.48 & 0.55 & 0.50 & 0.37 & 0.43 & 0.33 & 0.50 & 0.56 & 0.50 & 0.53 & 0.57 & 0.53 & 0.59 & 0.56 & 0.49 \\
\hline anneal & 0.99 & 0.99 & 0.99 & 0.99 & 0.99 & 0.99 & 0.99 & 1.00 & 0.99 & 1.00 & 1.00 & 0.99 & 1.00 & 1.00 & 1.00 \\
\hline arrhythmia & 0.57 & 0.56 & 0.65 & 0.56 & 0.58 & 0.47 & 0.60 & 0.53 & 0.56 & 0.46 & 0.62 & 0.79 & 0.57 & 0.52 & 0.49 \\
\hline audiology & 0.84 & 0.83 & 0.80 & 0.75 & 0.75 & 0.73 & 0.81 & 0.84 & 0.78 & 0.84 & 0.85 & 0.81 & 0.83 & 0.68 & 0.81 \\
\hline autos & 0.85 & 0.86 & 0.86 & 0.79 & 0.79 & 0.78 & 0.85 & 0.86 & 0.86 & 0.85 & 0.88 & 0.87 & 0.87 & 0.86 & 0.84 \\
\hline balance-scale & 0.85 & 0.82 & 0.85 & 0.82 & 0.68 & 0.81 & 0.85 & 0.82 & 0.85 & 0.87 & 0.88 & 0.85 & 0.87 & 0.77 & 0.85 \\
\hline breast-cancer & 0.76 & 0.71 & 0.72 & 0.76 & 0.74 & 0.75 & 0.75 & 0.80 & 0.74 & 0.80 & 0.81 & 0.76 & 0.75 & 0.76 & 0.73 \\
\hline breast-w & 0.97 & 0.96 & 0.97 & 0.96 & 0.96 & 0.95 & 0.97 & 0.97 & 0.96 & 0.96 & 0.97 & 0.97 & 0.97 & 0.97 & 0.96 \\
\hline bridges 1 & 0.60 & 0.74 & 0.60 & 0.61 & 0.69 & 0.67 & 0.65 & 0.73 & 0.65 & 0.65 & 0.73 & 0.56 & 0.70 & 0.77 & 0.66 \\
\hline bridges 2 & 0.60 & 0.72 & 0.54 & 0.60 & 0.72 & 0.64 & 0.58 & 0.73 & 0.57 & 0.65 & 0.73 & 0.56 & 0.59 & 0.72 & 0.60 \\
\hline car & 0.95 & 0.98 & 0.95 & 0.94 & 0.98 & 0.93 & 0.95 & 0.98 & 0.95 & 0.95 & 0.98 & 0.95 & 0.96 & 0.99 & 0.95 \\
\hline $\mathrm{cmc}$ & 0.66 & 0.69 & 0.65 & 0.61 & 0.63 & 0.60 & 0.66 & 0.69 & 0.66 & 0.68 & 0.70 & 0.66 & 0.69 & 0.70 & 0.66 \\
\hline colic & 0.82 & 0.85 & 0.88 & 0.81 & 0.82 & 0.67 & 0.75 & 0.82 & 0.81 & 0.73 & 0.88 & 0.87 & 0.77 & 0.77 & 0.68 \\
\hline column-2C & 0.88 & 0.90 & 0.88 & 0.88 & 0.89 & 0.88 & 0.88 & 0.89 & 0.88 & 0.89 & 0.89 & 0.88 & 0.89 & 0.90 & 0.88 \\
\hline column-3C & 0.89 & 0.87 & 0.89 & 0.87 & 0.87 & 0.87 & 0.89 & 0.89 & 0.89 & 0.89 & 0.89 & 0.89 & 0.90 & 0.86 & 0.89 \\
\hline credit-a & 0.89 & 0.88 & 0.89 & 0.88 & 0.89 & 0.88 & 0.89 & 0.90 & 0.89 & 0.89 & 0.90 & 0.89 & 0.89 & 0.89 & 0.89 \\
\hline credit-g & 0.80 & 0.78 & 0.80 & 0.78 & 0.81 & 0.78 & 0.80 & 0.84 & 0.80 & 0.81 & 0.84 & 0.80 & 0.82 & 0.79 & 0.80 \\
\hline cylinder-bands & 0.75 & 0.82 & 0.82 & 0.77 & 0.79 & 0.72 & 0.79 & 0.83 & 0.80 & 0.77 & 0.84 & 0.83 & 0.81 & 0.80 & 0.69 \\
\hline dermatology & 0.96 & 0.96 & 0.96 & 0.95 & 0.95 & 0.95 & 0.96 & 0.96 & 0.96 & 0.96 & 0.96 & 0.96 & 0.96 & 0.96 & 0.96 \\
\hline diabetes & 0.83 & 0.82 & 0.83 & 0.81 & 0.83 & 0.80 & 0.83 & 0.84 & 0.83 & 0.84 & 0.84 & 0.84 & 0.85 & 0.84 & 0.83 \\
\hline ecoli & 0.88 & 0.88 & 0.88 & 0.86 & 0.87 & 0.86 & 0.88 & 0.89 & 0.88 & 0.89 & 0.89 & 0.88 & 0.89 & 0.86 & 0.88 \\
\hline flags & 0.78 & 0.79 & 0.76 & 0.74 & 0.75 & 0.74 & 0.76 & 0.79 & 0.76 & 0.78 & 0.79 & 0.78 & 0.78 & 0.81 & 0.76 \\
\hline glass & 0.83 & 0.81 & 0.83 & 0.79 & 0.80 & 0.78 & 0.83 & 0.83 & 0.83 & 0.83 & 0.84 & 0.83 & 0.84 & 0.84 & 0.84 \\
\hline haberman & 0.78 & 0.73 & 0.78 & 0.76 & 0.77 & 0.75 & 0.78 & 0.79 & 0.78 & 0.79 & 0.80 & 0.77 & 0.80 & 0.76 & 0.79 \\
\hline & 0.86 & & 0.86 & 0.87 & & & & & & 0.86 & 0.87 & 0.86 & 0.86 & 0.86 & 0.86 \\
\hline heart-c & 0.85 & 0.82 & 0.85 & 0.83 & 0.83 & 0.82 & 0.85 & 0.86 & 0.85 & 0.85 & 0.87 & 0.85 & 0.86 & 0.85 & 0.85 \\
\hline heart-h & 0.84 & 0.85 & 0.84 & 0.84 & 0.84 & 0.83 & 0.79 & 0.81 & 0.78 & 0.85 & 0.85 & 0.83 & 0.75 & 0.80 & 0.72 \\
\hline heart-statlog & 0.85 & 0.84 & 0.85 & 0.84 & 0.84 & 0.84 & 0.85 & 0.87 & & 0.86 & & & & 0.87 & 0.85 \\
\hline hepatitis & 0.85 & 0.80 & 0.89 & 0.86 & 0.83 & 0.83 & 0.86 & 0.86 & 0.84 & 0.82 & 0.88 & 0.89 & 0.83 & 0.83 & 0.82 \\
\hline ionosphere & 0.94 & 0.94 & 0.94 & 0.93 & 0.93 & 0.93 & 0.94 & 0.94 & 0.94 & 0.94 & 0.94 & 0.94 & 0.95 & 0.94 & 0.94 \\
\hline kdd-synthetic & 0.93 & 0.90 & 0.93 & 0.90 & 0.88 & 0.91 & 0.93 & 0.90 & 0.93 & 0.93 & 0.91 & 0.96 & 0.93 & 0.88 & 0.93 \\
\hline labor & 0.78 & 0.80 & 0.79 & 0.85 & 0.78 & 0.77 & 0.78 & 0.83 & 0.76 & 0.72 & 0.76 & & 0.71 & 0.77 & 0.67 \\
\hline liver-disorders & 0.79 & 0.81 & 0.79 & 0.77 & 0.78 & 0.76 & 0.79 & 0.80 & 0.79 & 0.80 & 0.80 & 0.79 & 0.82 & 0.80 & 0.79 \\
\hline lung-cancer & 0.68 & 0.54 & 0.68 & 0.65 & 0.68 & 0.66 & 0.67 & 0.70 & 0.67 & 0.69 & 0.71 & 0.68 & 0.69 & 0.64 & 0.67 \\
\hline lymph & 0.85 & 0.87 & 0.85 & 0.83 & 0.84 & 0.85 & 0.85 & 0.88 & 0.85 & 0.86 & 0.88 & 0.85 & 0.85 & 0.89 & 0.85 \\
\hline mb-promot & 0.87 & & 0.86 & & & & 0.86 & 0.89 & & 0.86 & 0.89 & 0.28 & 0.87 & 0.88 & 0.86 \\
\hline meta.data & 0.24 & 0.21 & 0.28 & 0.12 & 0.16 & 0.08 & 0.25 & 0.32 & 0.28 & 0.24 & 0.35 & 0.86 & 0.32 & 0.28 & 0.23 \\
\hline morphological & 0.77 & 0.79 & 0.77 & 0.75 & 0.76 & 0.74 & 0.77 & 0.78 & 0.77 & 0.78 & 0.78 & 0.78 & 0.79 & 0.78 & 0.78 \\
\hline postoperative & 0.65 & 0.66 & 0.65 & 0.68 & 0.66 & 0.68 & 0.68 & 0.72 & 0.68 & 0.72 & 0.72 & 0.65 & 0.70 & 0.69 & 0.66 \\
\hline prima & 0.48 & & 0.47 & 0.43 & 0.46 & & 0.44 & 0.53 & & 0.50 & 0.50 & 0.48 & 0.51 & 0.54 & 0.43 \\
\hline readings- 2 & 1.00 & 1.00 & 1.00 & 1.00 & 1.00 & 1.00 & 1.00 & 1.00 & 1.00 & 1.00 & 1.00 & 1.00 & 1.00 & 1.00 & 1.00 \\
\hline readings-4 & 1.00 & 1.00 & 1.00 & 1.00 & 1.00 & 1.00 & 1.00 & 1.00 & 1.00 & 1.00 & 1.00 & 1.00 & 1.00 & 1.00 & 1.00 \\
\hline semeion & 0.94 & 0.94 & 0.94 & 0.93 & 0.94 & 0.93 & 0.94 & 0.94 & 0.94 & 0.94 & 0.94 & 0.97 & 0.94 & 0.94 & 0.94 \\
\hline shuttle-control & 0.63 & & 0.57 & 0.52 & 0.49 & 0.53 & 0.58 & 0.6 & 0.48 & 0.58 & 0.60 & 0.63 & 0.56 & 0.56 & 0.55 \\
\hline sick & 0.99 & 0.99 & 0.99 & 0.99 & 0.99 & 0.98 & 0.99 & 0.99 & 0.99 & 0.98 & 0.99 & 0.99 & 0.99 & 0.99 & 0.98 \\
\hline solar-flare-1 & 0.77 & 0.76 & 0.76 & 0.73 & 0.75 & 0.73 & 0.76 & 0.77 & 0.76 & 0.77 & 0.77 & 0.76 & 0.77 & 0.77 & 0.76 \\
\hline solar-flare-2 & 0.77 & 0.76 & 0.76 & & 0.76 & 0.75 & 0.76 & 0.78 & 0.76 & 0.78 & 0.78 & 0.77 & 0.77 & 0.78 & 0.76 \\
\hline sonar & 0.88 & 0.84 & 0.88 & 0.85 & 0.84 & 0.85 & 0.88 & 0.87 & 0.88 & 0.88 & 0.87 & 0.88 & 0.88 & 0.83 & 0.88 \\
\hline soybean & 0.88 & 0.85 & 0.90 & 0.90 & 0.87 & 0.91 & 0.85 & 0.89 & 0.88 & 0.90 & 0.87 & 0.93 & 0.90 & 0.87 & 0.89 \\
\hline sponge & 0.95 & 0.92 & 0.94 & 0.94 & 0.91 & 0.92 & 0.94 & 0.96 & 0.94 & 0.96 & 0.96 & 0.95 & 0.94 & 0.94 & 0.93 \\
\hline tae & 0.72 & 0.75 & 0.72 & 0.67 & 0.64 & 0.67 & 0.72 & 0.76 & 0.72 & 0.74 & 0.77 & 0.72 & 0.76 & 0.75 & 0.73 \\
\hline tempdiag & 1.00 & & 1.00 & & & & & & & 1.00 & 1.00 & 1.00 & 1.00 & 1.00 & 1.00 \\
\hline tep.fea & 0.61 & 0.61 & 0.61 & 0.61 & 0.61 & 0.61 & 0.61 & 0.61 & 0.61 & 0.61 & 0.61 & 0.61 & 0.61 & 0.61 & 0.61 \\
\hline tic-tac-toe & 0.91 & 0.95 & 0.90 & 0.89 & 0.95 & 0.89 & 0.91 & 0.94 & 0.90 & 0.91 & 0.94 & 0.91 & 0.91 & 0.96 & 0.91 \\
\hline trains & 0.87 & & 0.88 & 0.78 & 0.61 & 0.79 & 0.77 & 0.80 & 0.75 & 0.88 & 0.79 & 0.85 & 0.66 & 0.56 & 0.71 \\
\hline transfusion & 0.79 & 0.75 & 0.79 & 0.78 & 0.78 & 0.78 & 0.79 & & 0.79 & 0.80 & 0.80 & 0.79 & 0.80 & 0.77 & 0.79 \\
\hline vehicle & 0.84 & 0.86 & 0.85 & 0.80 & 0.82 & 0.78 & 0.85 & 0.86 & 0.85 & 0.86 & 0.86 & 0.86 & 0.87 & 0.85 & 0.85 \\
\hline vote & 0.97 & 0.96 & 0.97 & 0.97 & 0.96 & 0.96 & 0.96 & 0.96 & 0.96 & 0.97 & 0.97 & 0.97 & 0.96 & 0.96 & 0.97 \\
\hline wine & 0.96 & & 0.96 & 0.95 & 0.94 & 0.95 & 0.96 & & 0.96 & 0.96 & 0.95 & 0.96 & 0.96 & 0.93 & 0.96 \\
\hline wine-red & 0.75 & 0.79 & 0.77 & 0.69 & 0.73 & 0.66 & 0.77 & 0.80 & 0.77 & 0.79 & 0.80 & 0.78 & 0.81 & 0.80 & 0.76 \\
\hline wine-white & 0.58 & 0.61 & 0.59 & 0.55 & 0.58 & 0.53 & 0.59 & 0.62 & 0.59 & 0.61 & 0.61 & 0.78 & 0.61 & 0.62 & 0.58 \\
\hline zoo & 0.90 & 0.90 & 0.84 & 0.94 & 0.95 & 0.94 & 0.84 & 0.96 & 0.84 & 0.90 & 0.91 & 0.90 & 0.75 & 0.96 & 0.81 \\
\hline Average Rank & 7.94 & 9.22 & 8.45 & 11.30 & 10.56 & 12.35 & 8.17 & 4.61 & 9.16 & 6.27 & 3.60 & 6.64 & 5.25 & 7.17 & 9.31 \\
\hline
\end{tabular}


Table 7.3: Values are the average performance (rank) of each version of HEAD-DT according to either accuracy or F-Measure.

\begin{tabular}{lccc}
\hline Version & $\begin{array}{c}\text { Accuracy } \\
\text { Rank }\end{array}$ & $\begin{array}{c}\text { F-Measure } \\
\text { Rank }\end{array}$ & Average \\
\hline ACC-A & 8.00 & 7.94 & 7.97 \\
ACC-M & 8.93 & 9.22 & 9.08 \\
ACC-H & 8.35 & 8.45 & 8.40 \\
AUC-A & 11.68 & 11.30 & 11.49 \\
AUC-M & 10.76 & 10.56 & 10.66 \\
AUC-H & 12.57 & 12.35 & 12.46 \\
FM-A & 8.25 & 8.17 & 8.21 \\
FM-M & 4.75 & 4.61 & 4.68 \\
FM-H & 9.10 & 9.16 & 9.13 \\
RAI-A & 6.41 & 6.27 & 6.34 \\
RAI-M & $\mathbf{3 . 7 2}$ & $\mathbf{3 . 6 0}$ & $\mathbf{3 . 6 6}$ \\
RAI-H & 6.64 & 6.64 & 6.64 \\
TPR-A & 4.93 & 5.25 & 5.09 \\
TPR-M & 6.88 & 7.17 & 7.03 \\
TPR-H & 9.04 & 9.31 & 9.18 \\
\hline
\end{tabular}

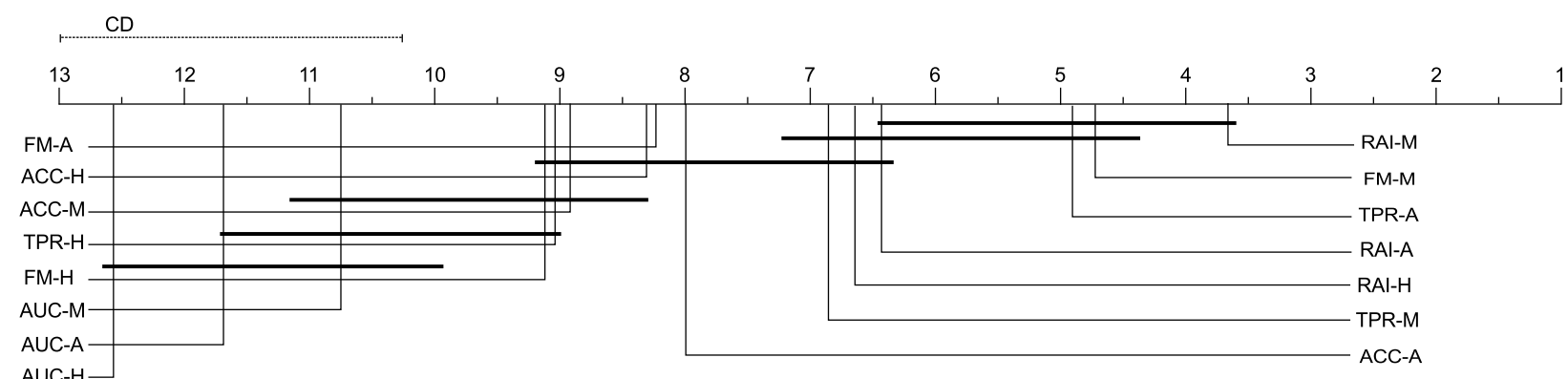

(a) Accuracy rank.

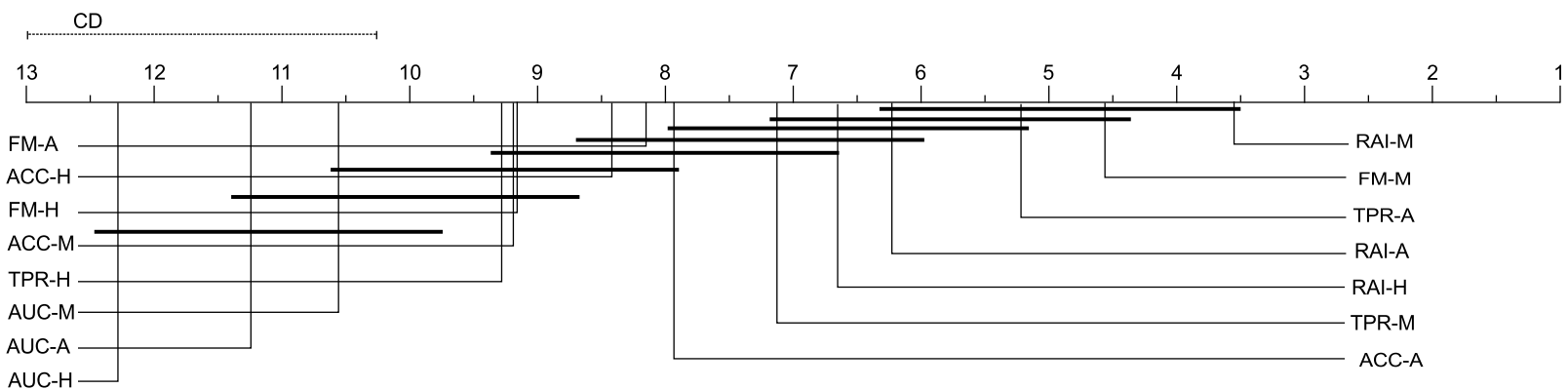

(b) F-Measure rank.

Figure 7.2: Critical diagrams for the balanced meta-training set experiment.

statistical significance in both evaluation measures, which is not the case of FM-M, TPR-A, and RAI-A.

Some interesting conclusions can be drawn from this first set of experiments with a balanced meta-training set:

- The AUC measure was not particularly effective for evolving decision-tree algorithms in this scenario, regardless of the aggregation scheme being used. Note that versions of HEAD-DT that employ AUC in their fitness function perform quite poorly when compared to the 
remaining versions - AUC-M, AUC-A, and AUC-H are in the bottom of the ranking: 13th, 14 th, and 15 th position, respectively;

- The use of the harmonic mean as an aggregation scheme was not successful overall. The harmonic mean was often worst aggregation scheme for the evaluation measures, occupying the lower positions of the ranking (except when combined to RAI).

- The use of the median, on the other hand, was shown to be very effective in most cases. For 3 evaluation measures the median was the best aggregation scheme (relative accuracy improvement, F-Measure, and AUC). In addition, the two best-ranked versions made use of the median as their aggregation scheme;

- The relative accuracy improvement was overall the best evaluation measure, occupying the top part of the ranking (1st, 4th, and 5th best-ranked versions);

- Finally, both F-Measure and recall were consistently among the best versions (2nd, 3rd, 6th, and 8th best-ranked versions), except once again when associated to the harmonic mean (11th and 12th).

Figure 7.3 depicts a picture of the fitness evolution throughout the evolutionary cycle. It presents both the best fitness from the population at a given generation and the average fitness from the corresponding generation.

Note that version AUC-M (Figure 7.3-(e)) achieves the perfect fitness from the very first generation $(A U C=1)$. We further analysed this particular case and verified that the decision-tree algorithm designed in this version does not perform any kind of pruning. Even though prune-free algorithms usually overfit the training data (if no pre-pruning is performed as well, they achieve $100 \%$ of accuracy in the training data) and thus underperform in the test data, it seems that this was not the case for the 5 data sets in the meta-training set. In the particular validation sets of the meta-training set, a prune-free algorithm with the stop criterion minimum number of 3 instances was capable of achieving perfect AUC. Nevertheless, this automatically-designed algorithm certainly suffered from overfitting in the meta-test set, since AUC-M was only the 13th-best out of 15 versions.

Versions FM-H (Figure 7.3-(i)) and TPR-H (Figure 7.3-(o)) also achieved their best fitness value in the first generation. The harmonic mean, due to its own nature (ignore higher values), seems to make the search for better individuals harder than the other aggregation schemes.

\subsubsection{Results for the Imbalanced Meta-Training Set}

We randomly selected 5 imbalanced data sets $(I R>10)$ from the $67 \mathrm{UCI}$ data sets described in Table 6.14 to be part of the meta-training set in this experiment: primary-tumor $(I R=$ $84)$, anneal $(I R=85.5)$, arrhythmia $(I R=122.5)$, winequality-white $(I R=439.6)$, and abalone $(I R=689)$. 


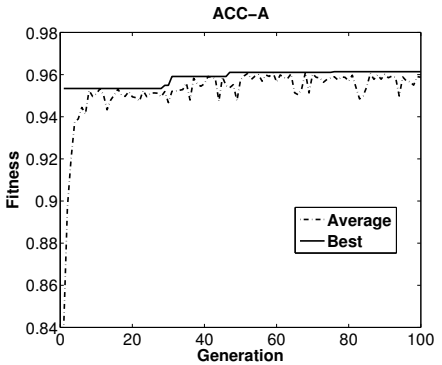

(a)

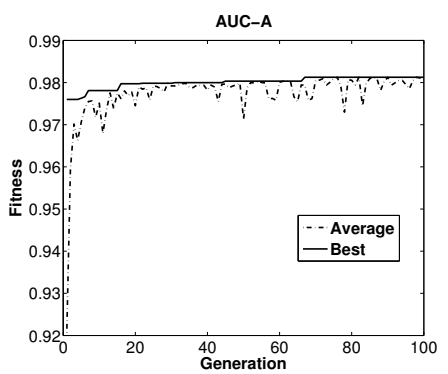

(d)

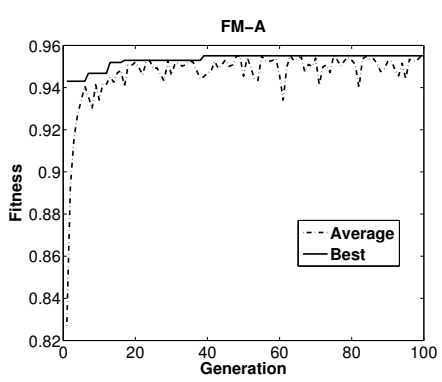

(g)

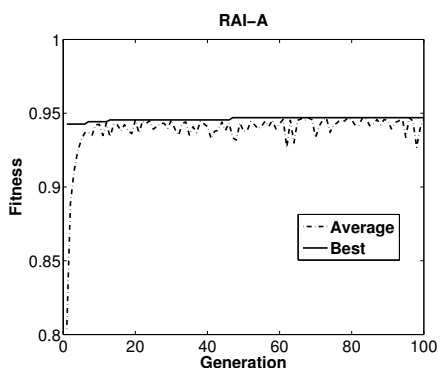

(j)

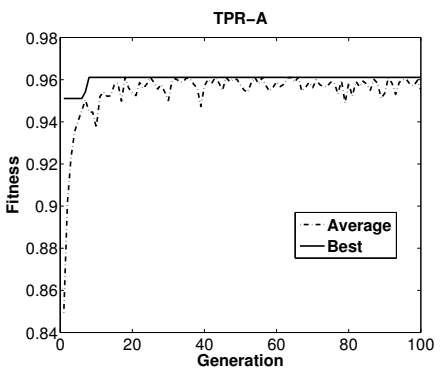

(m)

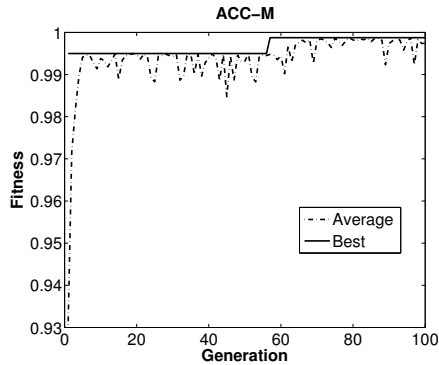

(b)

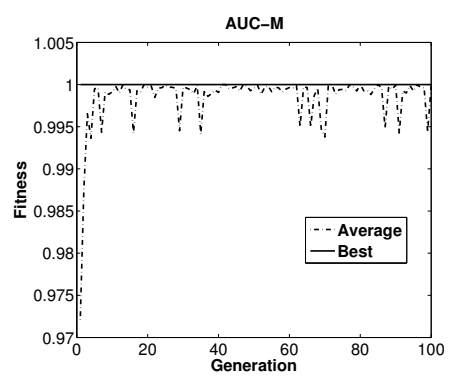

(e)

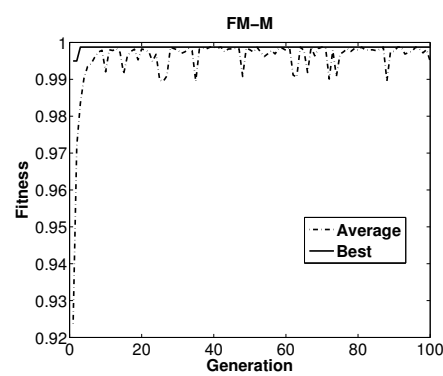

(h)

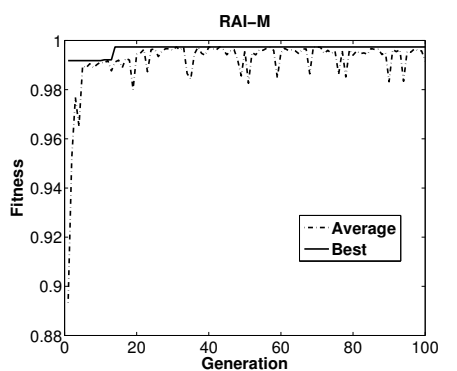

(k)

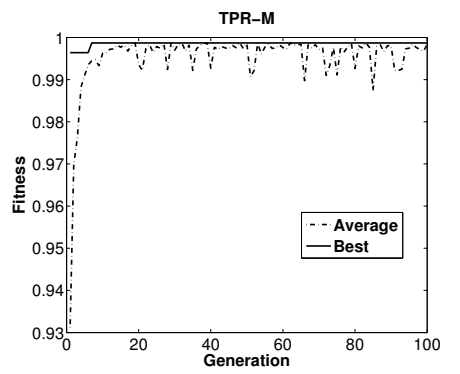

(n)

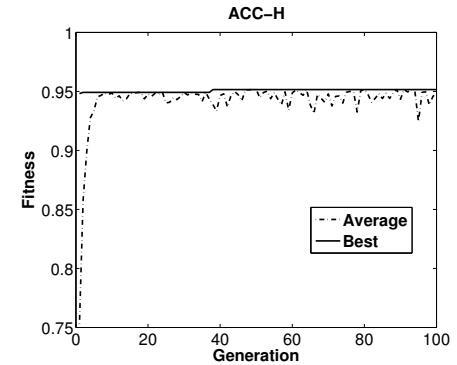

(c)

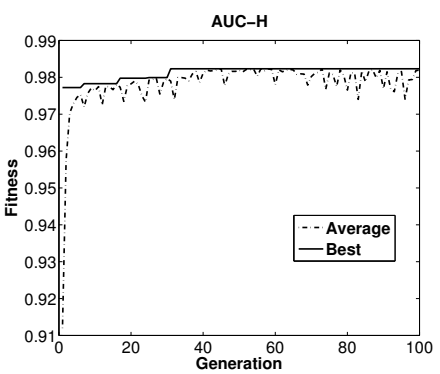

(f)

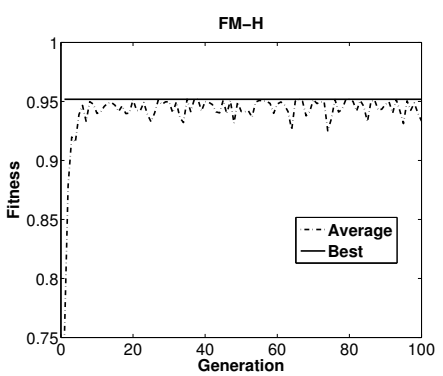

(i)

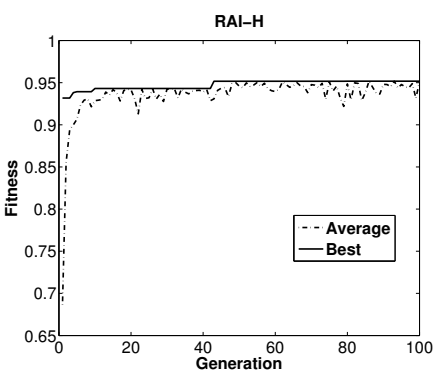

(l)

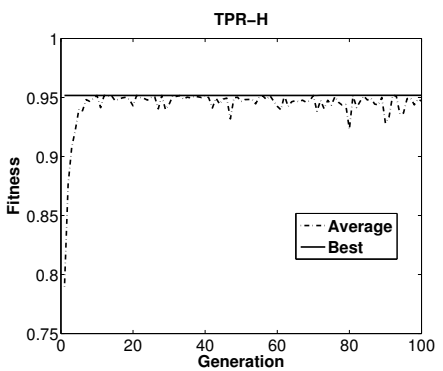

(o)

Figure 7.3: Fitness evolution in HEAD-DT for the balanced meta-training set. 
Tables 7.4 and 7.5 show the results for the 62 data sets in the meta-test set regarding accuracy and F-Measure, respectively. At the bottom of each table, the average rank is presented for the 15 versions of HEAD-DT created by varying the fitness functions. We once again did not present standard deviation values due to space limitations within the tables.

By careful inspection of both tables, we can see that the rankings in them are practically the same, with the average F-Measure being the best-ranked method for either evaluation measure. Only a small position-switching occurs between the accuracy and F-Measure rankings, with respect to the positions of ACC-H and RAI-M.

Table 7.6 summarizes the average rank values obtained by each version of HEAD-DT with respect to accuracy and F-Measure. Values in bold indicate the best performing version according to the corresponding evaluation measure. Note that version FM-A is the best-performing method regardless of the evaluation measure. The average of the average ranks (average across evaluation measures) indicates the following final ranking positions (from best to worst): 1) FM-A; 2) TPR-A; 3) TPR-H; 4) AUC-A; 5) AUC-H; 6) FM-H; 7) ACC-A; 8) ACC-M; 9) ACC-H; 10) RAI-M; 11) RAI-H; 12) FM-M; 13) TPR-M; 14) RAI-A; 15) AUC-M.

For evaluating whether the differences among the versions are statistically significant, we present the critical diagrams of the accuracy and F-Measure values in Figure 7.4. We can see that there are no statistically significant differences among the 7 (5) best-ranked versions regarding accuracy (F-Measure). In addition, note that the 6 best-ranked versions involve performance measures that are suitable for evaluating imbalanced problems (F-Measure, recall, and AUC), which is actually expected given the composition of the meta-training set.

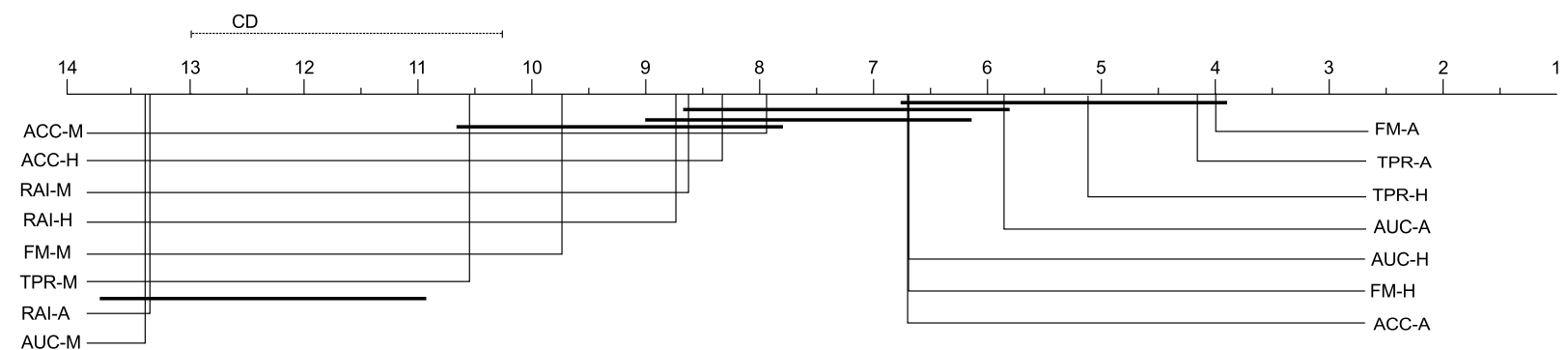

(a) Accuracy rank.

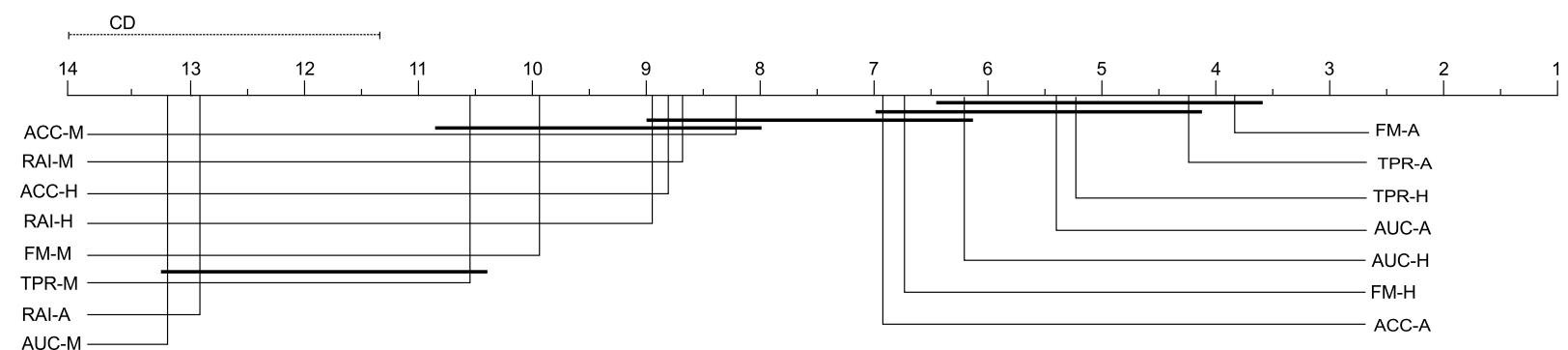

(b) F-Measure rank.

Figure 7.4: Critical diagrams for the imbalanced meta-training set experiment. 
Table 7.4: Accuracy values for the 15 versions of HEAD-DT varying the fitness functions. Meta-training comprises 5 imbalanced data sets.

\begin{tabular}{|c|c|c|c|c|c|c|c|c|c|c|c|c|c|c|c|}
\hline & $\begin{array}{c}\mathrm{ACC} \\
\mathrm{A}\end{array}$ & $\begin{array}{c}\text { ACC } \\
\text { M }\end{array}$ & $\begin{array}{c}\mathrm{ACC} \\
\mathrm{H}\end{array}$ & $\begin{array}{c}\mathrm{AUC} \\
\mathrm{A}\end{array}$ & $\begin{array}{c}\text { AUC } \\
\text { M }\end{array}$ & $\begin{array}{c}\text { AUC } \\
\mathrm{H}\end{array}$ & $\begin{array}{c}\text { FM } \\
\mathrm{A}\end{array}$ & $\begin{array}{c}\mathrm{FM} \\
\mathrm{M}\end{array}$ & $\begin{array}{c}\mathrm{FM} \\
\mathrm{H}\end{array}$ & $\begin{array}{c}\text { RAI } \\
\text { A }\end{array}$ & $\begin{array}{c}\text { RAI } \\
\text { M }\end{array}$ & $\begin{array}{c}\text { RAI } \\
\mathrm{H}\end{array}$ & $\begin{array}{c}\text { TPR } \\
\text { A }\end{array}$ & $\begin{array}{c}\text { TPR } \\
\text { M }\end{array}$ & $\begin{array}{c}\text { TPR } \\
\mathrm{H}\end{array}$ \\
\hline audiology & 0.67 & 0.65 & 0.69 & 0.75 & 0.60 & 0.60 & 0.59 & 0.61 & 0.60 & 0.59 & 0.59 & 0.55 & 0.60 & 0.59 & 0.60 \\
\hline autos & 0.79 & 0.74 & 0.72 & 0.84 & 0.63 & 0.76 & 0.74 & 0.49 & 0.55 & 0.44 & 0.47 & 0.71 & 0.77 & 0.35 & 0.69 \\
\hline balance-scale & 0.82 & 0.79 & 0.81 & 0.80 & 0.66 & 0.69 & 0.72 & 0.71 & 0.71 & 0.58 & 0.71 & 0.80 & 0.71 & 0.71 & 0.71 \\
\hline breast-cancer & 0.74 & 0.73 & 0.72 & 0.74 & 0.60 & 0.86 & 0.86 & 0.70 & 0.84 & 0.68 & 0.84 & 0.72 & 0.85 & 0.62 & 0.86 \\
\hline bridges 1 & 0.67 & 0.69 & 0.66 & 0.77 & 0.53 & 0.87 & 0.89 & 0.82 & 0.84 & 0.72 & 0.78 & 0.68 & 0.84 & 0.75 & 0.84 \\
\hline bridges 2 & 0.64 & 0.67 & 0.66 & 0.73 & 0.53 & 0.68 & 0.70 & 0.58 & 0.69 & 0.55 & 0.60 & 0.68 & 0.67 & 0.56 & 0.66 \\
\hline car & 0.94 & 0.92 & 0.94 & 0.91 & 0.76 & 1.00 & 1.00 & 0.94 & 1.00 & 0.80 & 1.00 & 0.93 & 1.00 & 0.94 & 1.00 \\
\hline heart-c & 0.80 & 0.80 & 0.80 & 0.83 & 0.67 & 0.76 & 0.79 & 0.75 & 0.76 & 0.64 & 0.76 & 0.80 & 0.78 & 0.74 & 0.79 \\
\hline $\mathrm{cmc}$ & 0.60 & 0.58 & 0.57 & 0.57 & 0.50 & 0.73 & 0.74 & 0.60 & 0.67 & 0.59 & 0.66 & 0.57 & 0.74 & 0.59 & 0.73 \\
\hline column-2C & 0.85 & 0.84 & 0.83 & 0.86 & 0.69 & 0.68 & 0.74 & 0.61 & 0.67 & 0.60 & 0.66 & 0.83 & 0.73 & 0.56 & 0.74 \\
\hline column-3C & 0.84 & 0.84 & 0.82 & 0.87 & 0.70 & 0.66 & 0.71 & 0.50 & 0.57 & 0.54 & 0.59 & 0.83 & 0.68 & 0.47 & 0.65 \\
\hline credit-a & 0.87 & 0.88 & 0.87 & 0.88 & 0.70 & 0.93 & 0.95 & 0.83 & 0.94 & 0.75 & 0.91 & 0.87 & 0.95 & 0.77 & 0.95 \\
\hline cylinder-bands & 0.78 & 0.74 & 0.72 & 0.78 & 0.62 & 0.77 & 0.81 & 0.72 & 0.76 & 0.64 & 0.77 & 0.73 & 0.80 & 0.68 & 0.82 \\
\hline dermatology & 0.96 & 0.95 & 0.93 & 0.96 & 0.73 & 0.86 & 0.88 & 0.84 & 0.87 & 0.69 & 0.84 & 0.92 & 0.88 & 0.84 & 0.88 \\
\hline ecoli & 0.84 & 0.85 & 0.84 & 0.86 & 0.68 & 0.87 & 0.88 & 0.85 & 0.85 & 0.69 & 0.84 & 0.84 & 0.88 & 0.84 & 0.88 \\
\hline flags & 0.72 & 0.68 & 0.64 & 0.71 & 0.56 & 0.96 & 0.95 & 0.92 & 0.94 & 0.75 & 0.91 & 0.66 & 0.95 & 0.92 & 0.94 \\
\hline credit-g & 0.76 & 0.75 & 0.75 & 0.77 & 0.63 & 0.74 & 0.79 & 0.70 & 0.78 & 0.62 & 0.73 & 0.75 & 0.78 & 0.70 & 0.77 \\
\hline glass & 0.79 & 0.79 & 0.75 & 0.78 & 0.62 & 0.84 & 0.87 & 0.77 & 0.84 & 0.68 & 0.80 & 0.76 & 0.86 & 0.71 & 0.85 \\
\hline haberman & 0.77 & 0.75 & 0.75 & 0.76 & 0.62 & 0.85 & 0.86 & 0.82 & 0.86 & 0.69 & 0.85 & 0.76 & 0.87 & 0.83 & 0.87 \\
\hline hayes-roth & 0.85 & 0.84 & 0.78 & 0.85 & 0.60 & 0.73 & 0.77 & 0.73 & 0.73 & 0.61 & 0.74 & 0.81 & 0.76 & 0.72 & 0.75 \\
\hline heart-statlog & 0.82 & 0.81 & 0.80 & 0.82 & 0.67 & 0.93 & 0.94 & 0.92 & 0.95 & 0.76 & 0.94 & 0.80 & 0.94 & 0.92 & 0.93 \\
\hline hepatitis & 0.81 & 0.81 & 0.81 & 0.89 & 0.68 & 0.89 & 0.86 & 0.81 & 0.82 & 0.66 & 0.80 & 0.80 & 0.86 & 0.81 & 0.85 \\
\hline colic & 0.87 & 0.85 & 0.85 & 0.86 & 0.67 & 0.79 & 0.84 & 0.79 & 0.84 & 0.67 & 0.80 & 0.86 & 0.82 & 0.79 & 0.82 \\
\hline heart-h & 0.82 & 0.81 & 0.81 & 0.82 & 0.66 & 0.82 & 0.85 & 0.82 & 0.82 & 0.68 & 0.82 & 0.81 & 0.84 & 0.83 & 0.85 \\
\hline ionosphere & 0.93 & 0.91 & 0.91 & 0.92 & 0.73 & 0.79 & 0.79 & 0.78 & 0.78 & 0.62 & 0.77 & 0.92 & 0.81 & 0.78 & 0.80 \\
\hline iris & 0.95 & 0.95 & 0.95 & 0.96 & 0.77 & 0.82 & 0.84 & 0.81 & 0.82 & 0.67 & 0.81 & 0.95 & 0.83 & 0.80 & 0.84 \\
\hline kr-vs-kp & 0.96 & 0.96 & 0.96 & 0.96 & 0.78 & 0.73 & 0.74 & 0.72 & 0.72 & 0.59 & 0.73 & 0.96 & 0.75 & 0.72 & 0.74 \\
\hline labor & 0.85 & 0.77 & 0.83 & 0.86 & 0.68 & 0.83 & 0.83 & 0.79 & 0.80 & 0.65 & 0.80 & 0.78 & 0.83 & 0.79 & 0.83 \\
\hline liver-disorders & 0.71 & 0.74 & 0.71 & 0.75 & 0.61 & 0.83 & 0.85 & 0.70 & 0.83 & 0.66 & 0.73 & 0.73 & 0.84 & 0.69 & 0.80 \\
\hline lung-cancer & 0.62 & 0.65 & 0.55 & 0.65 & 0.47 & 0.72 & 0.74 & 0.62 & 0.74 & 0.58 & 0.66 & 0.64 & 0.74 & 0.60 & 0.73 \\
\hline lymph & 0.84 & 0.80 & 0.80 & 0.85 & 0.65 & 0.85 & 0.89 & 0.78 & 0.83 & 0.70 & 0.83 & 0.79 & 0.87 & 0.67 & 0.89 \\
\hline meta.data & 0.13 & 0.12 & 0.11 & 0.10 & 0.13 & 0.78 & 0.83 & 0.77 & 0.81 & 0.66 & 0.79 & 0.11 & 0.82 & 0.78 & 0.82 \\
\hline morphological & 0.74 & 0.73 & 0.73 & 0.73 & 0.60 & 0.95 & 0.96 & 0.95 & 0.96 & 0.77 & 0.94 & 0.73 & 0.97 & 0.94 & 0.96 \\
\hline mb-promoters & 0.86 & 0.85 & 0.80 & 0.88 & 0.63 & 0.96 & 0.96 & 0.95 & 0.96 & 0.77 & 0.95 & 0.85 & 0.96 & 0.96 & 0.96 \\
\hline mushroom & 0.99 & 0.98 & 0.99 & 0.99 & 0.80 & 0.86 & 0.88 & 0.85 & 0.86 & 0.70 & 0.86 & 0.99 & 0.87 & 0.82 & 0.86 \\
\hline diabetes & 0.81 & 0.78 & 0.78 & 0.78 & 0.64 & 0.87 & 0.95 & 0.86 & 0.95 & 0.75 & 0.87 & 0.78 & 0.95 & 0.80 & 0.95 \\
\hline postoperative & 0.72 & 0.71 & 0.71 & 0.70 & 0.57 & 0.86 & 0.85 & 0.79 & 0.78 & 0.66 & 0.78 & 0.71 & 0.86 & 0.77 & 0.83 \\
\hline segment & 0.96 & 0.94 & 0.95 & 0.94 & 0.77 & 0.86 & 0.90 & 0.86 & 0.88 & 0.71 & 0.88 & 0.95 & 0.90 & 0.79 & 0.89 \\
\hline semeion & 0.94 & 0.92 & 0.93 & 0.94 & 0.76 & 0.10 & 0.22 & 0.09 & 0.13 & 0.17 & 0.13 & 0.93 & 0.22 & 0.08 & 0.18 \\
\hline readings- 2 & 0.94 & 0.98 & 1.00 & 1.00 & 0.80 & 0.92 & 0.94 & 0.90 & 0.93 & 0.75 & 0.92 & 0.95 & 0.94 & 0.88 & 0.94 \\
\hline readings-4 & 0.94 & 0.98 & 1.00 & 1.00 & 0.80 & 0.92 & 0.97 & 0.91 & 0.96 & 0.77 & 0.95 & 0.95 & 0.96 & 0.79 & 0.97 \\
\hline shuttle-control & 0.60 & 0.61 & 0.61 & 0.57 & 0.61 & 0.87 & 0.98 & 0.92 & 0.97 & 0.78 & 0.93 & 0.60 & 0.98 & 0.84 & 0.98 \\
\hline sick & 0.97 & 0.95 & 0.98 & 0.98 & 0.79 & 0.75 & 0.79 & 0.69 & 0.70 & 0.58 & 0.59 & 0.97 & 0.81 & 0.59 & 0.78 \\
\hline solar-flare-1 & 0.72 & 0.73 & 0.73 & 0.74 & 0.60 & 0.75 & 0.77 & 0.75 & 0.75 & 0.61 & 0.75 & 0.73 & 0.76 & 0.74 & 0.76 \\
\hline solar-flare2 & 0.76 & 0.75 & 0.76 & 0.75 & 0.61 & 0.72 & 0.76 & 0.72 & 0.75 & 0.60 & 0.72 & 0.75 & 0.77 & 0.72 & 0.76 \\
\hline sonar & 0.80 & 0.80 & 0.79 & 0.84 & 0.67 & 1.00 & 0.95 & 1.00 & 0.94 & 0.75 & 0.98 & 0.80 & 1.00 & 1.00 & 1.00 \\
\hline soybean & 0.79 & 0.88 & 0.83 & 0.87 & 0.67 & 0.58 & 0.63 & 0.57 & 0.62 & 0.49 & 0.58 & 0.68 & 0.62 & 0.57 & 0.62 \\
\hline sponge & 0.93 & 0.93 & 0.92 & 0.95 & 0.74 & 0.77 & 0.83 & 0.75 & 0.81 & 0.65 & 0.74 & 0.92 & 0.83 & 0.75 & 0.82 \\
\hline kdd-synthetic & 0.92 & 0.92 & 0.91 & 0.95 & 0.74 & 0.62 & 0.74 & 0.62 & 0.71 & 0.57 & 0.64 & 0.91 & 0.75 & 0.60 & 0.74 \\
\hline tae & 0.66 & 0.62 & 0.59 & 0.70 & 0.53 & 0.77 & 0.83 & 0.74 & 0.81 & 0.65 & 0.72 & 0.61 & 0.82 & 0.69 & 0.81 \\
\hline tempdiag & 1.00 & 1.00 & 0.91 & 1.00 & 0.77 & 0.77 & 0.81 & 0.75 & 0.78 & 0.64 & 0.76 & 0.95 & 0.81 & 0.75 & 0.81 \\
\hline tep.fea & 0.65 & 0.65 & 0.65 & 0.65 & 0.52 & 0.81 & 0.80 & 0.79 & 0.82 & 0.63 & 0.81 & 0.65 & 0.93 & 0.58 & 0.90 \\
\hline tic-tac-toe & 0.90 & 0.88 & 0.88 & 0.90 & 0.73 & 1.00 & 0.95 & 1.00 & 0.94 & 0.75 & 0.98 & 0.90 & 1.00 & 1.00 & 1.00 \\
\hline trains & 0.59 & 0.48 & 0.37 & 0.75 & 0.39 & 0.65 & 0.65 & 0.65 & 0.65 & 0.52 & 0.65 & 0.51 & 0.65 & 0.65 & 0.65 \\
\hline transfusion & 0.77 & 0.77 & 0.79 & 0.79 & 0.64 & 0.95 & 0.94 & 0.90 & 0.93 & 0.74 & 0.92 & 0.78 & 0.95 & 0.90 & 0.94 \\
\hline vehicle & 0.79 & 0.76 & 0.74 & 0.76 & 0.64 & 0.94 & 0.96 & 0.93 & 0.95 & 0.76 & 0.93 & 0.74 & 0.96 & 0.94 & 0.96 \\
\hline vote & 0.96 & 0.96 & 0.96 & 0.96 & 0.77 & 0.98 & 0.98 & 0.97 & 0.98 & 0.78 & 0.96 & 0.96 & 0.99 & 0.98 & 0.98 \\
\hline vowel & 0.73 & 0.75 & 0.74 & 0.67 & 0.62 & 0.96 & 0.96 & 0.95 & 0.96 & 0.76 & 0.95 & 0.70 & 0.95 & 0.95 & 0.95 \\
\hline wine & 0.93 & 0.90 & 0.90 & 0.96 & 0.75 & 0.94 & 0.99 & 0.95 & 0.98 & 0.78 & 0.94 & 0.91 & 0.99 & 0.91 & 0.99 \\
\hline wine-red & 0.69 & 0.65 & 0.63 & 0.62 & 0.55 & 0.99 & 1.00 & 0.99 & 1.00 & 0.80 & 0.98 & 0.63 & 1.00 & 0.98 & 1.00 \\
\hline breast-w & 0.95 & 0.94 & 0.95 & 0.95 & 0.76 & 0.94 & 0.97 & 0.95 & 0.97 & 0.78 & 0.95 & 0.95 & 0.98 & 0.95 & 0.97 \\
\hline zoo & 0.94 & 0.92 & 0.89 & 0.93 & 0.65 & 0.66 & 0.82 & 0.65 & 0.79 & 0.61 & 0.70 & 0.92 & 0.88 & 0.64 & 0.87 \\
\hline Average Rank & 6.70 & 7.94 & 8.40 & 5.87 & 13.43 & 6.70 & 4.02 & 9.71 & 6.70 & 13.40 & 8.58 & 8.72 & 4.19 & 10.53 & 5.10 \\
\hline
\end{tabular}


Table 7.5: F-Measure values for the 15 versions of HEAD-DT varying the fitness functions. Meta-training comprises 5 imbalanced data sets.

\begin{tabular}{|c|c|c|c|c|c|c|c|c|c|c|c|c|c|c|c|}
\hline & $\begin{array}{c}\mathrm{ACC} \\
\mathrm{A}\end{array}$ & $\begin{array}{c}\mathrm{ACC} \\
\mathrm{M}\end{array}$ & $\begin{array}{c}\mathrm{ACC} \\
\mathrm{H}\end{array}$ & $\begin{array}{c}\text { AUC } \\
\text { A }\end{array}$ & $\begin{array}{c}\text { AUC } \\
\mathrm{M}\end{array}$ & $\begin{array}{c}\text { AUC } \\
\mathrm{H}\end{array}$ & $\begin{array}{c}\text { FM } \\
\text { A }\end{array}$ & $\begin{array}{c}\text { FM } \\
\text { M }\end{array}$ & $\begin{array}{c}\text { FM } \\
\mathrm{H}\end{array}$ & $\begin{array}{c}\text { RAI } \\
\text { A }\end{array}$ & $\begin{array}{c}\text { RAI } \\
\text { M }\end{array}$ & $\begin{array}{c}\text { RAI } \\
\mathrm{H}\end{array}$ & $\begin{array}{c}\text { TPR } \\
\mathrm{A}\end{array}$ & $\begin{array}{c}\text { TPR } \\
\mathrm{M}\end{array}$ & $\begin{array}{c}\text { TPR } \\
\mathrm{H}\end{array}$ \\
\hline audiology & 0.63 & 0.60 & 0.64 & 0.71 & 0.56 & 0.58 & 0.54 & 0.51 & 0.52 & 0.52 & 0.50 & 0.48 & 0.48 & 0.46 & 0.47 \\
\hline autos & 0.79 & 0.74 & 0.71 & 0.84 & 0.63 & 0.76 & 0.73 & 0.46 & 0.54 & 0.43 & 0.45 & 0.71 & 0.76 & 0.30 & 0.68 \\
\hline balance-scale & 0.81 & 0.76 & 0.77 & 0.78 & 0.65 & 0.66 & 0.67 & 0.59 & 0.63 & 0.53 & 0.61 & 0.77 & 0.60 & 0.59 & 0.61 \\
\hline breast-cancer & 0.68 & 0.67 & 0.68 & 0.73 & 0.59 & 0.86 & 0.86 & 0.67 & 0.84 & 0.68 & 0.84 & 0.65 & 0.85 & 0.62 & 0.86 \\
\hline bridges 1 & 0.62 & 0.63 & 0.60 & 0.77 & 0.51 & 0.87 & 0.89 & 0.81 & 0.82 & 0.71 & 0.75 & 0.63 & 0.83 & 0.70 & 0.83 \\
\hline bridges 2 & 0.57 & 0.60 & 0.59 & 0.72 & 0.50 & 0.68 & 0.70 & 0.58 & 0.69 & 0.55 & 0.60 & 0.61 & 0.67 & 0.56 & 0.66 \\
\hline car & 0.93 & 0.92 & 0.94 & 0.91 & 0.76 & 1.00 & 1.00 & 0.94 & 1.00 & 0.80 & 1.00 & 0.93 & 1.00 & 0.94 & 1.00 \\
\hline heart-c & 0.80 & 0.80 & 0.80 & 0.83 & 0.67 & 0.74 & 0.78 & 0.69 & 0.70 & 0.62 & 0.71 & 0.80 & 0.76 & 0.69 & 0.77 \\
\hline $\mathrm{cmc}$ & 0.59 & 0.57 & 0.57 & 0.57 & 0.49 & 0.73 & 0.73 & 0.53 & 0.61 & 0.58 & 0.59 & 0.57 & 0.72 & 0.53 & 0.71 \\
\hline column-2C & 0.85 & 0.84 & 0.83 & 0.86 & 0.69 & 0.67 & 0.73 & 0.52 & 0.62 & 0.58 & 0.59 & 0.83 & 0.71 & 0.48 & 0.72 \\
\hline column-3C & 0.83 & 0.84 & 0.82 & 0.87 & 0.70 & 0.66 & 0.71 & 0.40 & 0.53 & 0.54 & 0.57 & 0.82 & 0.68 & 0.40 & 0.65 \\
\hline credit-a & 0.87 & 0.88 & 0.87 & 0.88 & 0.70 & 0.93 & 0.95 & 0.78 & 0.94 & 0.75 & 0.89 & 0.87 & 0.95 & 0.73 & 0.95 \\
\hline cylinder-bands & 0.78 & 0.73 & 0.72 & 0.78 & 0.62 & 0.77 & 0.81 & 0.70 & 0.75 & 0.63 & 0.76 & 0.73 & 0.80 & 0.67 & 0.81 \\
\hline dermatology & 0.96 & 0.95 & 0.93 & 0.96 & 0.73 & 0.86 & 0.88 & 0.84 & 0.87 & 0.69 & 0.84 & 0.91 & 0.88 & 0.84 & 0.87 \\
\hline ecoli & 0.83 & 0.84 & 0.83 & 0.85 & 0.68 & 0.87 & 0.88 & 0.85 & 0.84 & 0.69 & 0.84 & 0.83 & 0.88 & 0.83 & 0.88 \\
\hline flags & 0.70 & 0.67 & 0.62 & 0.71 & 0.56 & 0.96 & 0.95 & 0.92 & 0.94 & 0.75 & 0.91 & 0.63 & 0.95 & 0.92 & 0.94 \\
\hline credit-g & 0.72 & 0.74 & 0.72 & 0.76 & 0.62 & 0.74 & 0.79 & 0.69 & 0.78 & 0.62 & 0.72 & 0.73 & 0.78 & 0.69 & 0.77 \\
\hline glass & 0.78 & 0.78 & 0.74 & 0.77 & 0.61 & 0.84 & 0.87 & 0.75 & 0.84 & 0.68 & 0.79 & 0.74 & 0.85 & 0.70 & 0.85 \\
\hline haberman & 0.71 & 0.71 & 0.70 & 0.75 & 0.61 & 0.84 & 0.86 & 0.80 & 0.86 & 0.68 & 0.84 & 0.70 & 0.86 & 0.81 & 0.86 \\
\hline hayes-roth & 0.85 & 0.83 & 0.78 & 0.85 & 0.59 & 0.72 & 0.76 & 0.68 & 0.68 & 0.60 & 0.70 & 0.81 & 0.74 & 0.68 & 0.73 \\
\hline heart-statlog & 0.82 & 0.81 & 0.80 & 0.82 & 0.67 & 0.93 & 0.93 & 0.88 & 0.94 & 0.75 & 0.92 & 0.80 & 0.93 & 0.89 & 0.89 \\
\hline hepatitis & 0.76 & 0.75 & 0.76 & 0.88 & 0.66 & 0.88 & 0.85 & 0.76 & 0.77 & 0.64 & 0.76 & 0.74 & 0.84 & 0.76 & 0.83 \\
\hline colic & 0.87 & 0.85 & 0.85 & 0.86 & 0.66 & 0.76 & 0.83 & 0.77 & 0.83 & 0.67 & 0.77 & 0.86 & 0.80 & 0.77 & 0.80 \\
\hline heart-h & 0.82 & 0.80 & 0.80 & 0.82 & 0.66 & 0.82 & 0.85 & 0.82 & 0.82 & 0.68 & 0.82 & 0.81 & 0.84 & 0.83 & 0.84 \\
\hline ionosphere & 0.93 & 0.91 & 0.91 & 0.92 & 0.73 & 0.77 & 0.78 & 0.73 & 0.74 & 0.62 & 0.73 & 0.92 & 0.78 & 0.76 & 0.78 \\
\hline iris & 0.95 & 0.95 & 0.95 & 0.96 & 0.77 & 0.82 & 0.84 & 0.80 & 0.82 & 0.67 & 0.81 & 0.95 & 0.83 & 0.80 & 0.84 \\
\hline kr-vs-kp & 0.96 & 0.96 & 0.96 & 0.96 & 0.78 & 0.72 & 0.73 & 0.69 & 0.70 & 0.58 & 0.70 & 0.96 & 0.73 & 0.70 & 0.72 \\
\hline labor & 0.83 & 0.72 & 0.83 & 0.85 & 0.68 & 0.83 & 0.83 & 0.79 & 0.80 & 0.65 & 0.79 & 0.74 & 0.83 & 0.79 & 0.83 \\
\hline liver-disorders & 0.69 & 0.73 & 0.70 & 0.75 & 0.61 & 0.83 & 0.85 & 0.69 & 0.83 & 0.66 & 0.73 & 0.72 & 0.84 & 0.68 & 0.80 \\
\hline lung-cancer & 0.60 & 0.64 & 0.48 & 0.65 & 0.42 & 0.71 & 0.74 & 0.59 & 0.73 & 0.57 & 0.64 & 0.63 & 0.73 & 0.56 & 0.72 \\
\hline lymph & 0.83 & 0.80 & 0.78 & 0.85 & 0.65 & 0.85 & 0.89 & 0.77 & 0.83 & 0.70 & 0.83 & 0.78 & 0.87 & 0.67 & 0.89 \\
\hline meta.data & 0.11 & 0.10 & 0.09 & 0.09 & 0.12 & 0.78 & 0.83 & 0.77 & 0.81 & 0.65 & 0.78 & 0.08 & 0.82 & 0.77 & 0.82 \\
\hline morphological & 0.72 & 0.71 & 0.72 & 0.72 & 0.60 & 0.95 & 0.96 & 0.95 & 0.96 & 0.77 & 0.94 & 0.72 & 0.97 & 0.94 & 0.96 \\
\hline mb-promoters & 0.86 & 0.84 & 0.80 & 0.88 & 0.63 & 0.96 & 0.96 & 0.95 & 0.96 & 0.77 & 0.95 & 0.85 & 0.96 & 0.96 & 0.96 \\
\hline mushroom & 0.99 & 0.98 & 0.99 & 0.99 & 0.80 & 0.86 & 0.88 & 0.85 & 0.86 & 0.70 & 0.86 & 0.99 & 0.87 & 0.82 & 0.86 \\
\hline diabetes & 0.80 & 0.78 & 0.77 & 0.78 & 0.64 & 0.87 & 0.95 & 0.86 & 0.94 & 0.75 & 0.86 & 0.77 & 0.95 & 0.80 & 0.95 \\
\hline postoperative & 0.63 & 0.59 & 0.59 & 0.65 & 0.51 & 0.86 & 0.85 & 0.79 & 0.78 & 0.66 & 0.78 & 0.59 & 0.86 & 0.77 & 0.83 \\
\hline segment & 0.96 & 0.94 & 0.95 & 0.94 & 0.77 & 0.85 & 0.90 & 0.86 & 0.88 & 0.71 & 0.88 & 0.95 & 0.90 & 0.78 & 0.89 \\
\hline semeion & 0.94 & 0.90 & 0.92 & 0.93 & 0.76 & 0.09 & 0.21 & 0.07 & 0.11 & 0.16 & 0.11 & 0.92 & 0.21 & 0.05 & 0.17 \\
\hline readings-2 & 0.93 & 0.98 & 1.00 & 1.00 & 0.80 & 0.92 & 0.94 & 0.89 & 0.93 & 0.75 & 0.92 & 0.94 & 0.94 & 0.88 & 0.94 \\
\hline readings-4 & 0.93 & 0.98 & 1.00 & 1.00 & 0.80 & 0.92 & 0.97 & 0.91 & 0.96 & 0.77 & 0.95 & 0.94 & 0.96 & 0.79 & 0.97 \\
\hline shuttle-control & 0.52 & 0.49 & 0.47 & 0.55 & 0.52 & 0.87 & 0.98 & 0.92 & 0.97 & 0.78 & 0.92 & 0.47 & 0.98 & 0.83 & 0.98 \\
\hline sick & 0.97 & 0.94 & 0.98 & 0.98 & 0.79 & 0.71 & 0.77 & 0.63 & 0.68 & 0.56 & 0.54 & 0.96 & 0.79 & 0.51 & 0.75 \\
\hline solar-flare-1 & 0.71 & 0.71 & 0.71 & 0.72 & 0.59 & 0.74 & 0.76 & 0.73 & 0.73 & 0.60 & 0.73 & 0.70 & 0.75 & 0.72 & 0.74 \\
\hline solar-flare2 & 0.74 & 0.73 & 0.73 & 0.74 & 0.60 & 0.72 & 0.76 & 0.70 & 0.74 & 0.60 & 0.72 & 0.73 & 0.77 & 0.69 & 0.76 \\
\hline sonar & 0.80 & 0.80 & 0.79 & 0.84 & 0.67 & 1.00 & 0.93 & 1.00 & 0.93 & 0.74 & 0.97 & 0.80 & 1.00 & 1.00 & 1.00 \\
\hline soybean & 0.77 & 0.87 & 0.81 & 0.86 & 0.66 & 0.57 & 0.63 & 0.57 & 0.62 & 0.49 & 0.58 & 0.65 & 0.62 & 0.57 & 0.61 \\
\hline sponge & 0.91 & 0.91 & 0.88 & 0.94 & 0.73 & 0.76 & 0.83 & 0.74 & 0.81 & 0.65 & 0.74 & 0.88 & 0.83 & 0.74 & 0.82 \\
\hline kdd-synthetic & 0.92 & 0.92 & 0.91 & 0.95 & 0.74 & 0.61 & 0.74 & 0.60 & 0.71 & 0.56 & 0.63 & 0.91 & 0.75 & 0.57 & 0.73 \\
\hline tae & 0.66 & 0.61 & 0.59 & 0.70 & 0.53 & 0.76 & 0.83 & 0.73 & 0.81 & 0.65 & 0.72 & 0.61 & 0.82 & 0.69 & 0.80 \\
\hline tempdiag & 1.00 & 1.00 & 0.91 & 1.00 & 0.77 & 0.76 & 0.81 & 0.71 & 0.75 & 0.64 & 0.75 & 0.95 & 0.81 & 0.73 & 0.80 \\
\hline tep.fea & 0.61 & 0.61 & 0.60 & 0.61 & 0.49 & 0.79 & 0.78 & 0.77 & 0.80 & 0.61 & 0.78 & 0.61 & 0.93 & 0.51 & 0.89 \\
\hline tic-tac-toe & 0.90 & 0.88 & 0.88 & 0.91 & 0.73 & 1.00 & 0.93 & 1.00 & 0.93 & 0.74 & 0.97 & 0.90 & 1.00 & 1.00 & 1.00 \\
\hline trains & 0.59 & 0.47 & 0.33 & 0.75 & 0.37 & 0.61 & 0.61 & 0.61 & 0.61 & 0.49 & 0.61 & 0.49 & 0.61 & 0.61 & 0.61 \\
\hline transfusion & 0.73 & 0.71 & 0.77 & 0.77 & 0.63 & 0.96 & 0.94 & 0.90 & 0.93 & 0.74 & 0.92 & 0.73 & 0.95 & 0.90 & 0.94 \\
\hline vehicle & 0.79 & 0.75 & 0.74 & 0.76 & 0.64 & 0.93 & 0.96 & 0.92 & 0.95 & 0.76 & 0.92 & 0.73 & 0.96 & 0.94 & 0.96 \\
\hline vote & 0.96 & 0.96 & 0.96 & 0.96 & 0.77 & 0.98 & 0.98 & 0.97 & 0.98 & 0.78 & 0.96 & 0.96 & 0.99 & 0.98 & 0.98 \\
\hline vowel & 0.73 & 0.75 & 0.74 & 0.66 & 0.62 & 0.96 & 0.96 & 0.95 & 0.96 & 0.76 & 0.95 & 0.69 & 0.95 & 0.95 & 0.95 \\
\hline wine & 0.93 & 0.90 & 0.90 & 0.96 & 0.75 & 0.94 & 0.99 & 0.95 & 0.98 & 0.78 & 0.94 & 0.91 & 0.99 & 0.91 & 0.99 \\
\hline wine-red & 0.68 & 0.63 & 0.61 & 0.61 & 0.54 & 0.99 & 1.00 & 0.99 & 1.00 & 0.80 & 0.98 & 0.61 & 1.00 & 0.98 & 1.00 \\
\hline breast-w & 0.95 & 0.94 & 0.95 & 0.95 & 0.76 & 0.94 & 0.97 & 0.95 & 0.97 & 0.78 & 0.95 & 0.95 & 0.98 & 0.95 & 0.97 \\
\hline zoo & 0.94 & 0.91 & 0.86 & 0.93 & 0.62 & 0.65 & 0.82 & 0.65 & 0.79 & 0.61 & 0.69 & 0.91 & 0.88 & 0.64 & 0.87 \\
\hline Average Rank & 6.92 & 8.23 & 8.74 & 5.44 & 13.23 & 6.25 & 3.83 & 9.97 & 6.79 & 12.94 & 8.65 & 8.95 & 4.27 & 10.56 & 5.25 \\
\hline
\end{tabular}


Table 7.6: Values are the average performance (rank) of each version of HEAD-DT according to either accuracy or F-Measure.

\begin{tabular}{lccc}
\hline Version & $\begin{array}{c}\text { Accuracy } \\
\text { Rank }\end{array}$ & $\begin{array}{c}\text { F-Measure } \\
\text { Rank }\end{array}$ & Average \\
\hline ACC-A & 6.70 & 6.92 & 6.81 \\
ACC-M & 7.94 & 8.23 & 8.09 \\
ACC-H & 8.40 & 8.74 & 8.57 \\
AUC-A & 5.87 & 5.44 & 5.66 \\
AUC-M & 13.43 & 13.23 & 13.33 \\
AUC-H & 6.70 & 6.25 & 6.48 \\
FM-A & $\mathbf{4 . 0 2}$ & $\mathbf{3 . 8 3}$ & $\mathbf{3 . 9 3}$ \\
FM-M & 9.71 & 9.97 & 9.84 \\
FM-H & 6.70 & 6.79 & 6.75 \\
RAI-A & 13.40 & 12.94 & 13.17 \\
RAI-M & 8.58 & 8.65 & 8.62 \\
RAI-H & 8.72 & 8.95 & 8.84 \\
TPR-A & 4.19 & 4.27 & 4.23 \\
TPR-M & 10.53 & 10.56 & 10.55 \\
TPR-H & 5.10 & 5.25 & 5.18 \\
\hline
\end{tabular}

The following conclusions can be drawn from this second set of experiments concerning imbalanced data sets:

- The relative accuracy improvement is not suitable for dealing with imbalanced data sets and hence occupies the bottom positions of the ranking (10th, 11th, and 14th positions). This behavior is expected given that RAI measures the improvement over the majority-class accuracy, and such an improvement is often damaging for imbalanced problems, in which the goal is to improve the accuracy of the less-frequent class(es);

- The median was the worst aggregation scheme overall, figuring in the bottom positions of the ranking (8th, 10th, 12th, 13th, and 15th). It is interesting to notice that the median was very successful in the balanced meta-training experiment, and quite the opposite in the imbalanced one;

- The simple average, on the other hand, presented itself as the best aggregation scheme for the imbalanced data, figuring in the top of the ranking (1st, 2nd, 4th, 7th), except when associated to RAI (14th), which was the worst performance measure overall;

- The 6 best-ranked versions were those employing performance measures known to be suitable for imbalanced data (F-Measure, recall, and AUC);

- Finally, the harmonic mean had a solid performance throughout this experiment, differently from its performance in the balanced meta-training experiment.

Figure 7.5 depicts a picture of the fitness evolution throughout the evolutionary cycle. Note that whereas some versions find their best individual at the very end of evolution (e.g., FM-H, Figure 7.5-(i)), others converge quite early (e.g., TPR-H, Figure 7.5-(o)), though there seems to exist no direct relation between early (or late) convergence and predictive performance. 


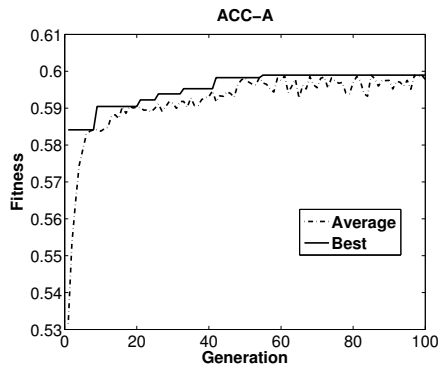

(a)

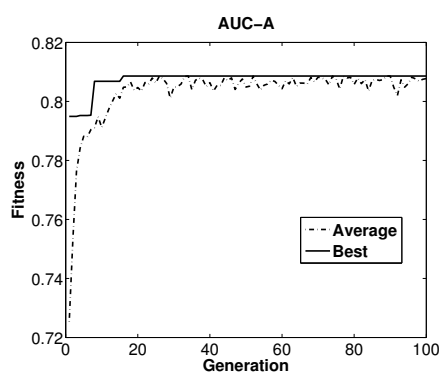

(d)

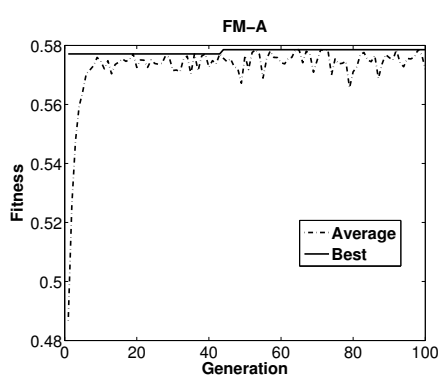

(g)

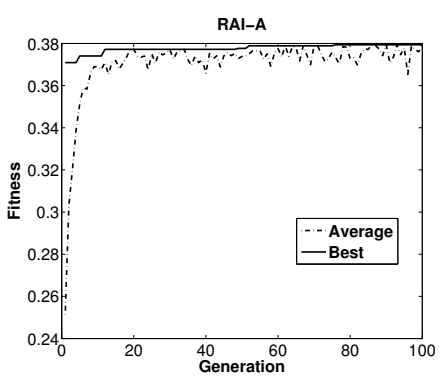

(j)

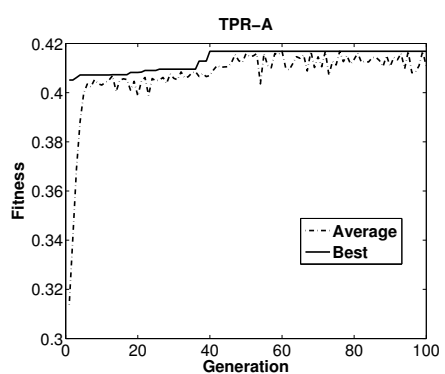

(m)

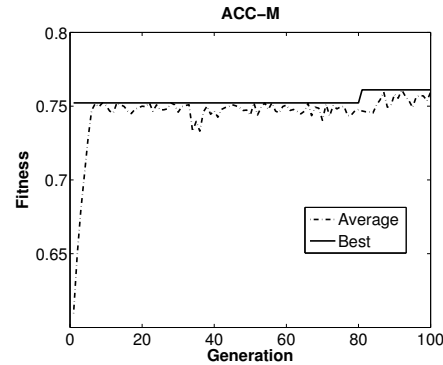

(b)

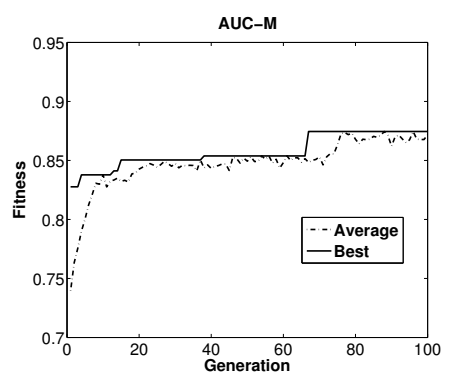

(e)

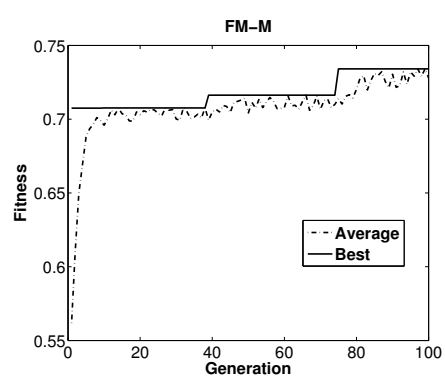

(h)

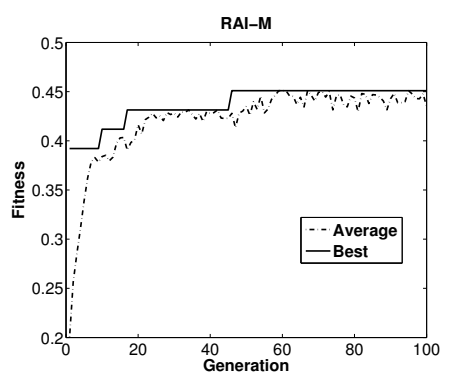

(k)

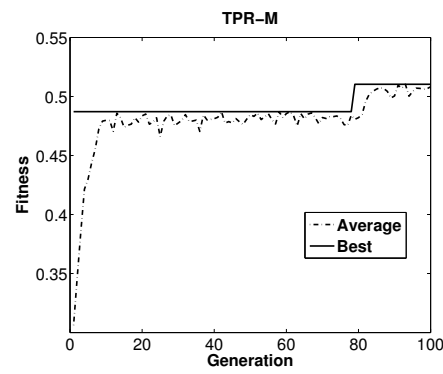

(n)

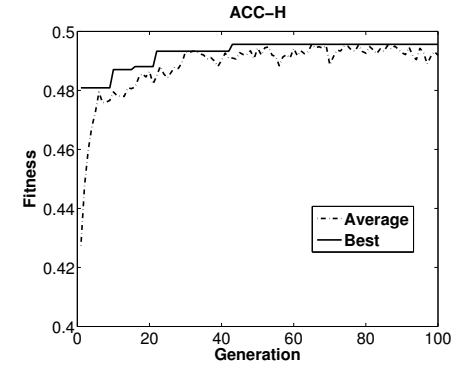

(c)

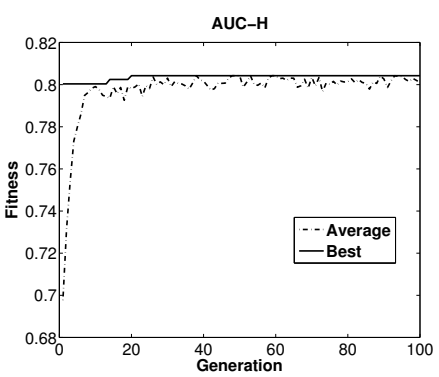

(f)

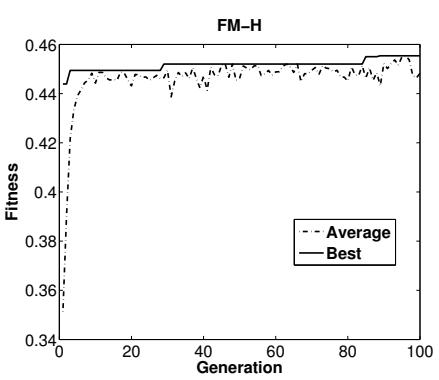

(i)

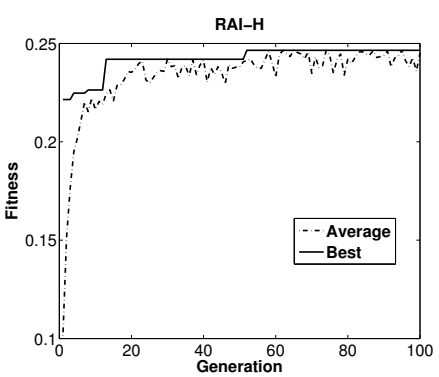

(l)

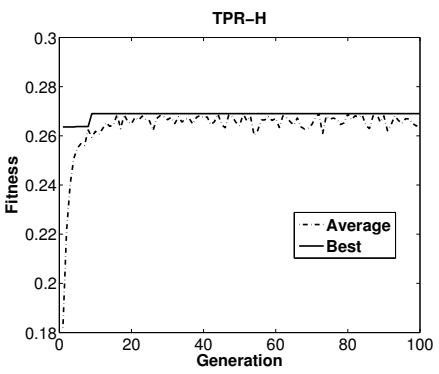

(o)

Figure 7.5: Fitness evolution in HEAD-DT for the imbalanced meta-training set. 


\subsubsection{Experiments with the Best-Performing Strategy}

Considering that the median of the relative accuracy improvement (RAI-M) was the best-ranked fitness function for the balanced meta-training set, and that the average F-Measure (FM-A) was the best-ranked fitness function for the imbalanced meta-training set, we perform a comparison of these HEAD-DT versions with the baseline decision-tree induction algorithms C4.5, CART, and REPTree.

For version RAI-M, we use the same meta-training set as before: iris $(I R=1)$, segment $(I R=$ $1)$, vowel $(I R=1)$, mushroom $(I R=1.07)$, and kr-vs-kp $(I R=1.09)$. The resulting algorithm is tested over the 10 most-balanced data sets from Table 6.14:

1. meta-data $(I R=1)$;

2. mfeat $(I R=1)$;

3. mb-promoters $(I R=1)$;

4. kdd-synthetic $(I R=1)$;

5. trains $(I R=1)$;

6. tae $(I R=1.06)$;

7. vehicle $(I R=1.10)$;

8. $\operatorname{sonar}(I R=1.14)$;

9. heart-c $(I R=1.20)$;

10. credit-a $(I R=1.25)$.

For version FM-A, we also use the same meta-training set as before: primary-tumor $(I R=$ $84)$, anneal $(I R=85.5)$, arrhythmia $(I R=122.5)$, winequality-white $(I R=439.6)$, and abalone $(I R=689)$. The resulting algorithm is tested over the 10 most-imbalanced data sets from Table 6.14:

- flags $(I R=15)$;

- $\operatorname{sick}(I R=15.33)$;

- $\operatorname{car}(I R=18.62)$;

- $\operatorname{autos}(I R=22.33)$;

- sponge $(I R=23.33)$;

- postoperative $(I R=32)$; 
- $\operatorname{lymph}(I R=40.50)$;

- $\operatorname{audiology}(I R=57)$;

- winequality-red $(I R=68.10)$;

- $\operatorname{ecoli}(I R=71.50)$.

In Chapter 6, we saw that HEAD-DT is capable of generating effective algorithms tailored to a particular application domain (gene expression data). Now, with this new experiment, our goal is to verify whether HEAD-DT is capable of generating effective algorithms tailored to a particular statistical profile - in this case, tailored to balanced/imbalanced data.

Table 7.7 shows the accuracy and F-Measure values for HEAD-DT, C4.5, CART, and REPTree, in the $20 \mathrm{UCI}$ data sets (10 most-balanced and 10 most-imbalanced). The version of HEAD-DT executed over the first 10 data sets is RAI-M, whereas the version executed over the remaining 10 is FM-A. In both versions, HEAD-DT is executed 5 times as usual, and the results are averaged.

Table 7.7: Accuracy and F-Measure values for the 10 most-balanced data sets and the 10 most-imbalanced data sets.

\begin{tabular}{|c|c|c|c|c|c|c|c|c|c|}
\hline \multirow{2}{*}{ Data Set } & \multirow{2}{*}{ IR } & \multicolumn{2}{|c|}{ HEAD-DT } & \multicolumn{2}{|c|}{ C4.5 } & \multicolumn{2}{|c|}{ CART } & \multicolumn{2}{|c|}{ REP } \\
\hline & & Accuracy & F-Measure & Accuracy & F-Measure & Accuracy & F-Measure & Accuracy & F-Measure \\
\hline meta.data & 1.00 & $\mathbf{0 . 3 5} \pm \mathbf{0 . 0 9}$ & $\mathbf{0 . 3 5} \pm \mathbf{0 . 1 0}$ & $0.04 \pm 0.03$ & $0.02 \pm 0.02$ & $0.05 \pm 0.03$ & $0.02 \pm 0.01$ & $0.04 \pm 0.00$ & $0.00 \pm 0.00$ \\
\hline mfeat & 1.00 & $\mathbf{0 . 7 9} \pm \mathbf{0 . 0 1}$ & $\mathbf{0 . 7 8} \pm \mathbf{0 . 0 2}$ & $0.72 \pm 0.02$ & $0.70 \pm 0.02$ & $0.72 \pm 0.04$ & $0.70 \pm 0.04$ & $0.72 \pm 0.03$ & $0.70 \pm 0.03$ \\
\hline mb-promoters & 1.00 & $\mathbf{0 . 8 9} \pm \mathbf{0 . 0 3}$ & $\mathbf{0 . 8 9} \pm \mathbf{0 . 0 3}$ & $0.80 \pm 0.13$ & $0.79 \pm 0.14$ & $0.72 \pm 0.14$ & $0.71 \pm 0.14$ & $0.77 \pm 0.15$ & $0.76 \pm 0.15$ \\
\hline kdd-synthetic & 1.00 & $0.91 \pm 0.03$ & $0.91 \pm 0.03$ & $0.91 \pm 0.04$ & $0.91 \pm 0.04$ & $0.88 \pm 0.04$ & $0.88 \pm 0.04$ & $0.88 \pm 0.03$ & $0.87 \pm 0.04$ \\
\hline trains & 1.00 & $0.79 \pm 0.06$ & $0.79 \pm 0.06$ & $0.90 \pm \mathbf{0 . 3 2}$ & $0.90 \pm \mathbf{0 . 3 2}$ & $0.20 \pm 0.42$ & $0.20 \pm 0.42$ & $0.00 \pm 0.00$ & $0.00 \pm 0.00$ \\
\hline tae & 1.06 & $\mathbf{0 . 7 7} \pm \mathbf{0 . 0 3}$ & $\mathbf{0 . 7 7} \pm \mathbf{0 . 0 3}$ & $0.60 \pm 0.11$ & $0.59 \pm 0.12$ & $0.51 \pm 0.12$ & $0.49 \pm 0.15$ & $0.47 \pm 0.12$ & $0.45 \pm 0.12$ \\
\hline vehicle & 1.10 & $0.86 \pm 0.01$ & $0.86 \pm 0.01$ & $0.74 \pm 0.04$ & $0.73 \pm 0.04$ & $0.72 \pm 0.04$ & $0.71 \pm 0.05$ & $0.71 \pm 0.04$ & $0.70 \pm 0.04$ \\
\hline sonar & 1.14 & $\mathbf{0 . 8 7} \pm \mathbf{0 . 0 1}$ & $\mathbf{0 . 8 7} \pm \mathbf{0 . 0 1}$ & $0.73 \pm 0.08$ & $0.72 \pm 0.08$ & $0.71 \pm 0.06$ & $0.71 \pm 0.06$ & $0.71 \pm 0.07$ & $0.70 \pm 0.07$ \\
\hline heart-c & 1.20 & $0.87 \pm \mathbf{0 . 0 1}$ & $\mathbf{0 . 8 7} \pm \mathbf{0 . 0 1}$ & $0.77 \pm 0.09$ & $0.76 \pm 0.09$ & $0.81 \pm 0.04$ & $0.80 \pm 0.04$ & $0.77 \pm 0.08$ & $0.77 \pm 0.08$ \\
\hline credit-a & 1.25 & $\mathbf{0 . 9 0} \pm \mathbf{0 . 0 1}$ & $\mathbf{0 . 9 0} \pm \mathbf{0 . 0 1}$ & $0.85 \pm 0.03$ & $0.85 \pm 0.03$ & $0.84 \pm 0.03$ & $0.84 \pm 0.03$ & $0.85 \pm 0.03$ & $0.85 \pm 0.03$ \\
\hline flags & 15.00 & $0.74 \pm 0.01$ & $0.74 \pm 0.01$ & $0.63 \pm 0.05$ & $0.61 \pm 0.05$ & $0.61 \pm 0.10$ & $0.57 \pm 0.10$ & $0.62 \pm 0.10$ & $0.58 \pm 0.10$ \\
\hline sick & 15.33 & $0.98 \pm 0.01$ & $0.98 \pm 0.01$ & $0.99 \pm 0.00$ & $0.99 \pm 0.00$ & $0.99 \pm 0.01$ & $0.99 \pm \mathbf{0 . 0 1}$ & $0.99 \pm 0.01$ & $0.99 \pm 0.01$ \\
\hline car & 18.62 & $0.98 \pm \mathbf{0 . 0 1}$ & $0.98 \pm 0.01$ & $0.93 \pm 0.02$ & $0.93 \pm 0.02$ & $0.97 \pm 0.02$ & $0.97 \pm 0.02$ & $0.89 \pm 0.02$ & $0.89 \pm 0.02$ \\
\hline autos & 22.33 & $0.85 \pm 0.03$ & $0.85 \pm 0.03$ & $0.86 \pm 0.06$ & $\mathbf{0 . 8 5} \pm \mathbf{0 . 0 7}$ & $0.78 \pm 0.10$ & $0.77 \pm 0.10$ & $0.65 \pm 0.08$ & $0.62 \pm 0.07$ \\
\hline sponge & 23.33 & $0.94 \pm 0.01$ & $0.93 \pm 0.02$ & $0.93 \pm 0.06$ & $0.89 \pm 0.09$ & $0.91 \pm 0.06$ & $0.88 \pm 0.09$ & $0.91 \pm 0.08$ & $0.88 \pm 0.10$ \\
\hline postoperative & 32.00 & $0.72 \pm \mathbf{0 . 0 1}$ & $0.67 \pm 0.04$ & $0.70 \pm 0.05$ & $0.59 \pm 0.07$ & $0.71 \pm 0.06$ & $0.59 \pm 0.08$ & $0.69 \pm 0.09$ & $0.58 \pm 0.09$ \\
\hline lymph & 40.50 & $0.87 \pm \mathbf{0 . 0 1}$ & $\mathbf{0 . 8 7} \pm \mathbf{0 . 0 1}$ & $0.78 \pm 0.09$ & $0.79 \pm 0.10$ & $0.75 \pm 0.12$ & $0.73 \pm 0.14$ & $0.77 \pm 0.11$ & $0.76 \pm 0.12$ \\
\hline audiology & 57.00 & $0.79 \pm 0.04$ & $\mathbf{0 . 7 7} \pm \mathbf{0 . 0 5}$ & $0.78 \pm 0.07$ & $0.75 \pm 0.08$ & $0.74 \pm 0.05$ & $0.71 \pm 0.05$ & $0.74 \pm 0.08$ & $0.70 \pm 0.09$ \\
\hline wine-red & 68.10 & $0.74 \pm 0.02$ & $0.74 \pm \mathbf{0 . 0 2}$ & $0.61 \pm 0.03$ & $0.61 \pm 0.03$ & $0.63 \pm 0.02$ & $0.61 \pm 0.02$ & $0.60 \pm 0.03$ & $0.58 \pm 0.03$ \\
\hline \multirow[t]{2}{*}{ ecoli } & 71.50 & $\mathbf{0 . 8 6} \pm \mathbf{0 . 0 1}$ & $\mathbf{0 . 8 6} \pm \mathbf{0 . 0 1}$ & $0.84 \pm 0.07$ & $0.83 \pm 0.07$ & $0.84 \pm 0.07$ & $0.82 \pm 0.07$ & $0.79 \pm 0.09$ & $0.77 \pm 0.09$ \\
\hline & Rank & 1.30 & 1.30 & 2.25 & 2.25 & 2.90 & 2.90 & 3.55 & 3.55 \\
\hline
\end{tabular}

Observe in Table 7.7 that HEAD-DT (RAI-M) outperforms C4.5, CART, and REPTree in 8 out of 10 data sets (in both accuracy and F-Measure), whereas C4.5 is the best algorithm in the remaining two data sets. The same can be said about HEAD-DT (FM-A), which also outperforms C4.5, CART, and REPTree in 8 out of 10 data sets, being outperformed once by C4.5 and once by CART.

We proceed by presenting the critical diagrams of accuracy and F-Measure (Figure 7.6) in order to evaluate whether the differences among the algorithms are statistically significant. Note 
that HEAD-DT is the best-ranked method, often in the 1st position (rank =1.30). HEAD-DT (versions RAI-M and FM-A) outperforms both CART and REPTree with statistical significance for $\alpha=0.05$. With respect to C4.5, it is outperformed by HEAD-DT with statistical significance for $\alpha=0.10$, though not for $\alpha=0.05$. Nevertheless, we are confident that being the best method in 16 out of 20 data sets is enough to conclude that HEAD-DT automatically generates decision-tree algorithms tailored to balanced/imbalanced data that are consistently more effective than C4.5, CART, and REPTree.

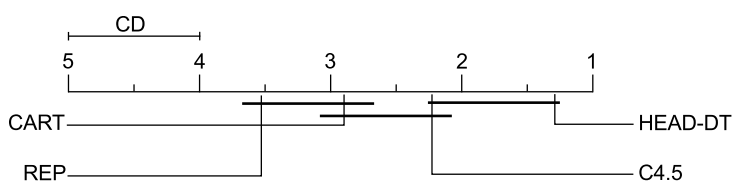

(a) Accuracy rank for the balanced data sets.

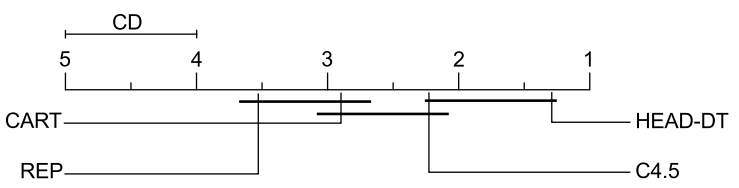

(b) F-Measure rank for the balanced data sets.

Figure 7.6: Critical diagrams for accuracy and F-Measure. Values are regarding the 20 UCI data sets in Table 7.7.

Since HEAD-DT is run 5 times for alleviating the randomness effect of evolutionary algorithms, we further analyse the 5 algorithms generated by HEAD-DT for the balanced meta-training set and the 5 algorithms generated for the imbalanced meta-training set.

Regarding the balanced meta-training set, we noticed that the favored split criterion was the $\mathrm{G}$ statistic (present in $40 \%$ of the algorithms). The favored stop criterion was stopping the tree-splitting process only when there is a single instance in the node (present in $80 \%$ of the algorithms). The homogeneous stop was present in the remaining $20 \%$ of the algorithms, but since a single instance is always homogeneous (only 1 class represented in the node), we can say that HEAD-DT stop criterion was actually stop splitting nodes when they are homogeneous. Surprisingly, the favored pruning strategy was not to use any pruning strategy $(80 \%$ of the algorithms). It seems that this particular combination of design components did not lead to overfitting, even though the trees were not pruned at any point. Algorithm 10 shows this custom algorithm designed for balanced data sets.

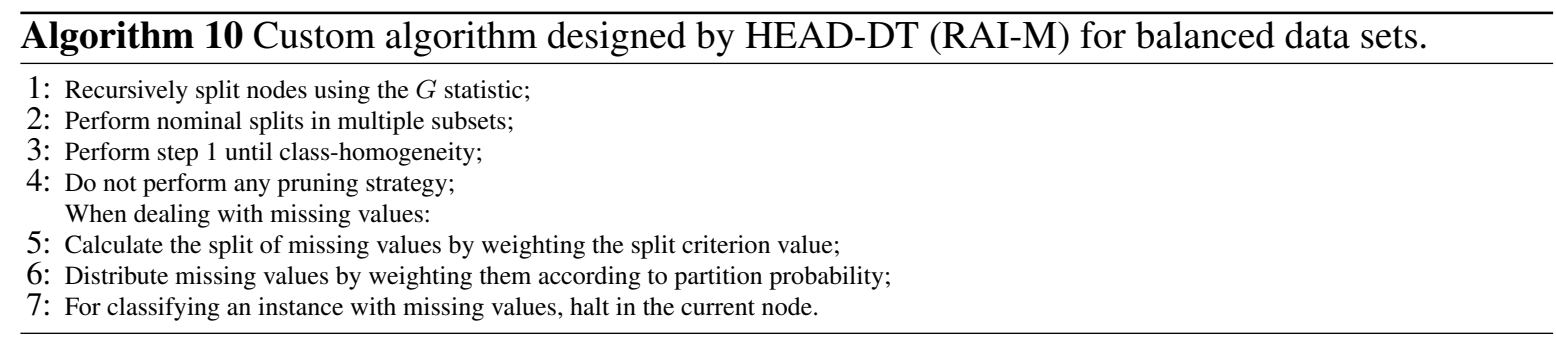

Regarding the imbalanced meta-training set, we noticed that two split criteria stand out: DCSM (present in $40 \%$ of the algorithms) and Normalized Gain (also present in $40 \%$ of the algorithms). In $100 \%$ of the algorithms, the nominal splits were aggregated into binary splits. The favored stop criterion was either the homogeneous stop (60\% of the algorithms) of the algorithms or tree 
stop when a maximum depth of around 10 levels is reached (40\% of the algorithms). Finally, the pruning strategy was also divided between PEP pruning with 1 SE (40\% of the algorithms) and no pruning at all ( $40 \%$ of the algorithms). We noticed that whenever the algorithm employed DCSM, PEP pruning was the favored pruning strategy. Similarly, whenever the Normalized Gain was selected, no pruning was the favored pruning strategy. It seems that HEAD-DT was capable of detecting a correlation between different split criteria and pruning strategies. Algorithm 11 shows the custom algorithm that was tailored to imbalanced data (we actually present the choices of different components when it was the case).

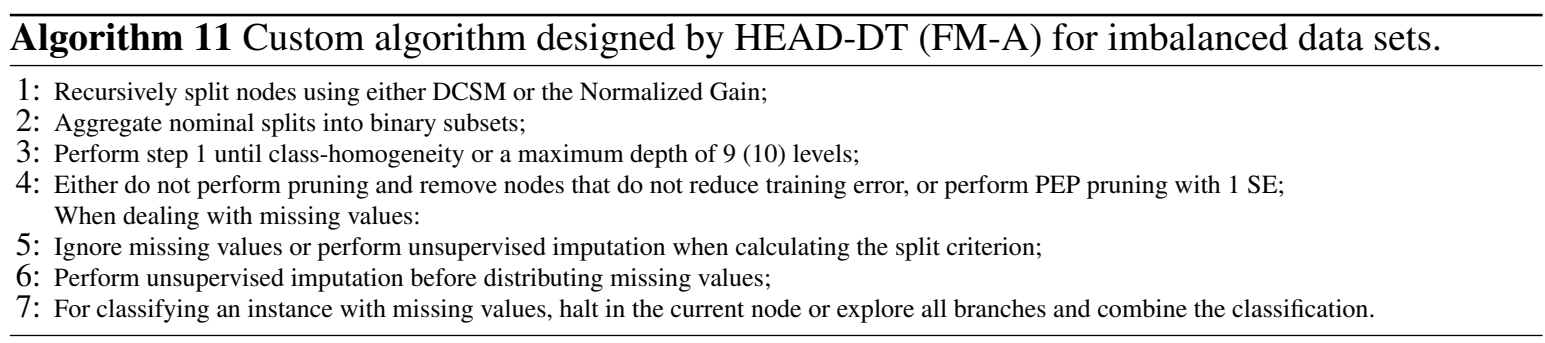

Regarding the missing value strategies, we did not notice any particular pattern in either the balanced or the imbalanced scenarios. Hence, the missing-value strategies presented in Algorithms 10 and 11 are only examples of selected components, though they did not stand out in terms of appearance frequency.

\subsection{Chapter Remarks}

In this chapter, we performed a series of experiments to analyse in more detail the impact of different fitness functions during the evolutionary cycle of HEAD-DT. In the first part of the chapter, we presented 5 classification performance measures and three aggregation schemes to combine these measures during fitness evaluation of multiple data sets. The combination of performance measures and aggregation schemes resulted in 15 different versions of HEAD-DT.

We designed two experimental scenarios to evaluate the 15 versions of HEAD-DT. In the first scenario, HEAD-DT is executed on a meta-training set with 5 balanced data sets, and on a meta-test set with the remaining 62 available UCI data sets. In the second scenario, HEAD-DT is executed on a meta-training set withs 5 imbalanced data sets, and the meta-test set with the remaining 62 available UCI data sets. For measuring the level of data set balance, we make use of the imbalance ratio $(I R)$, which is the ratio between the most-frequent and the less-frequent classes of the data.

Results of the experiments indicated that the median of the relative accuracy improvement was the most suitable fitness function for the balanced scenario, whereas the average of the F-Measure was the most suitable fitness function for the imbalanced scenario. The next step of the empirical analysis was to compare these versions of HEAD-DT with the baseline decision-tree induction algorithms C4.5, CART, and REPTree. For such, we employed the same meta-training sets than 
before, though the meta-test sets exclusively comprised balanced (imbalanced) data sets. The experimental results confirmed that HEAD-DT can generate algorithms tailored to a particular statistical profile (data set balance) that are more effective than C4.5, CART, and REPTree, outperforming them in 16 out of 20 data sets. 


\begin{tabular}{ll} 
CHAPTER \\
\hline \\
\hline
\end{tabular}

This thesis proposed and investigated a new hyper-heuristics approach for the automatic design of decision-tree induction algorithms, namely HEAD-DT (Hyper-Heuristic Evolutionary Algorithm for Automatically Designing Decision-Tree Induction Algorithms). HEAD-DT makes use of an evolutionary algorithm to perform a search in the space of more than 21 million decision-tree induction algorithms. The search is guided by the performance of the candidate algorithms in a meta-training set, and it may follow two distinct frameworks:

- Evolution of a decision-tree induction algorithm tailored to one specific data set at a time (specific framework);

- Evolution of a decision-tree induction algorithm from multiple data sets (general framework).

We carried out extensive experimentation with both specific and general frameworks. In the first, HEAD-DT uses data from a single data set in both meta-training and meta-test sets. The goal is to design a given decision-tree induction algorithm so it excels at that specific data set, and no requirements are made regarding its performance in other data sets. Experiments with 20 UCI data sets showed that HEAD-DT significantly outperforms algorithms like CART (Breiman et al., 1984) and C4.5 (Quinlan, 1993) with respect to performance measures such as accuracy and F-Measure.

In the second framework, HEAD-DT was further tested with three distinct objectives:

1. To evolve a single decision-tree algorithm tailor-made to data sets from a particular application domain (homogeneous approach). 
2. To evolve a single decision-tree algorithm robust across a variety of different data sets (heterogeneous approach).

3. To evolve a single decision-tree algorithm tailored to data sets that share a particular statistical profile.

In order to evaluate objective 1, we performed a thorough empirical analysis on 35 microarray gene expression data sets (Souto et al., 2008). The experimental results indicated that automatically-designed decision-tree induction algorithms tailored to a particular domain (in this case, microarray data) usually outperform traditional decision-tree algorithms like C4.5 and CART.

For evaluating objective 2, we conducted a thorough empirical analysis on 67 UCI public data sets (Frank and Asuncion, 2010). According to the experimental results, the automatically-designed "all-around" decision-tree induction algorithms, which are meant to be robust across very different data sets, presented a performance similar to traditional decision-tree algorithms like C4.5 and CART, even though they seemed to be suffering from meta-overfitting.

With respect to objective 3, we first performed an extensive analysis with 15 distinct fitness functions for HEAD-DT. We concluded that the best versions of HEAD-DT were able to automatically design decision-tree induction algorithms tailored to balanced (and imbalanced) data sets which consistently outperformed traditional algorithms like C4.5 and CART.

Overall, HEAD-DT presented a good performance in the four different investigated scenarios (one scenario regarding the specific framework and three scenarios regarding the general framework). Next, we present the limitations (Section 8.1) and future work possibilities (Section 8.2) we envision for continuing the study started in this thesis. In the end of this chapter, we include a list of publications from the author within the period of his PhD studies (2010-1013).

\subsection{Limitations}

HEAD-DT has the intrinsic disadvantage of evolutionary algorithms, which is a high execution time. Furthermore, it inherits the disadvantages of a hyper-heuristic approach, which is the need of evaluating several data sets in the meta-training set (at least in the general framework), also translating into additional execution time. However, we recall from Chapter 6, Section 6.4, that HEAD-DT may be seen as a fast method for automatically designing decision-tree algorithms. Even though it may take up to several hours to generate a tailor-made algorithm for a given application domain, its further application in data sets from the domain of interest is just as fast as most traditional top-down decision-tree induction algorithms. The cost of developing a new decision-tree algorithm from scratch to a particular domain, on the other hand, would be in the order of several months.

We believe that the main limitation in the current implementation of HEAD-DT is the meta-training set setup. In this thesis, we employed a random methodology to select data sets 
to be part of the meta-training set in the general framework. Even though randomly-generated meta-training sets still provided good results for the homogeneous approach in the general framework, we believe that automatic, intelligent construction of meta-training sets can significantly improve HEAD-DT's predictive performance. Some suggestions regarding this matter are discussed in the next section.

Finally, the meta-overfitting problem identified in Chapter 6, Section 6.2.3, is also a current limitation of the HEAD-DT implementation. Probably the easiest way of solving this problem would be to feed the meta-training set with a large variety of data sets. However, this solution would slow down evolution significantly, taking HEAD-DT's average execution time from a few hours to days or weeks. We comment in the next section some other alternatives to the meta-overfitting problem.

\subsection{Opportunities for Future Work}

In this section, we discuss seven new research opportunities resulting from this study, namely: i) proposing an extended HEAD-DT's genome that takes into account new induction strategies, oblique splits, and the ability to deal with regression problems; ii) proposing a new multi-objective fitness function; iii) proposing a new method for automatically selecting proper data sets to be part of the meta-training set; iv) proposing a parameter-free evolutionary search; v) proposing solutions to the meta-overfitting problem; vi) proposing the evolution of decision-tree induction algorithms to compose an ensemble; and vii) proposing a new genetic search that makes use of grammar-based techniques. These new research directions are discussed next in detail.

\subsubsection{Extending HEAD-DT's Genome: New Induction Strategies, Oblique Splits, Regression Problems}

HEAD-DT can be naturally extended so its genome accounts for induction strategies other than the top-down induction. For instance, an induction gene could be responsible for choosing among the following approaches for tree induction: top-down induction (currently implemented), bottom-up induction (following the work of Barros et al. (2011b, 2013) and Landeweerd et al. (1983)), beam-search induction (following the work of Basgalupp et al. (2011)), and possibly a hybrid approach that combines the three previous strategies.

Furthermore, one can think of a new gene to be included among the split genes, in which an integer would index the following split options: univariate splits (currently implemented), oblique splits (along with several parameter values that would determine the strategy for generating the oblique split), and omni splits (real-time decision for each split as whether it should be univariate or oblique, following the work of Yildiz and Alpaydin (2001)). 
Finally, HEAD-DT could be adapted to regression problems. For such, split measures specially designed to regression have to be implemented - see, for instance, Chapter 2, Section 2.3.1.

\subsubsection{Multi-Objective Fitness Function}

In Chapter 7, we tested 15 different single-objective fitness functions for HEAD-DT. A natural extension regarding fitness evaluation would be to employ multi-objective strategies to account for multiple objectives being simultaneously optimised. For instance, instead of searching only for the largest average F-Measure achieved by a candidate algorithm in the meta-training set, HEAD-DT could look for an algorithm that induces trees with reduced size. Considering that, for similar predictive performance, a simpler model is always preferred (as stated by the well-known Occam's razor principle), it makes sense to optimise both a measure of predictive performance (such as F-Measure) and a measure of model complexity (such as tree size).

Possible solutions for dealing with multi-objective optimisation include the Pareto dominance approach and the lexicographic analysis (Freitas, 2004). The first assumes the set of non-dominated solutions is provided to the user (instead of a single best solution). Hence, the evolutionary algorithm must be modified as to properly handle the selection operation, as well as elitism and the return of the final solution. A lexicographic approach, in turn, assumes that each objective has a different priority order, and thus it decides which individual is best by traversing the objectives from the highest to the lowest priority. Each multi-objective strategy has advantages and disadvantages, and an interesting research effort would be to compare a number of strategies, so one could see how they cope with different optimisation goals.

\subsubsection{Automatic Selection of the Meta-Training Set}

In this thesis, we employed a methodology that randomly selected data sets to be part of the meta-training set. Since the performance of the evolved decision-tree algorithm is directly related to the data sets that belong to the meta-training set, we believe an intelligent and automatic strategy to select a proper meta-training set would be beneficial to the final user. For instance, the user could provide HEAD-DT with a list of available data sets, and HEAD-DT would automatically select from this list those data sets which are more similar to the available meta-test set.

Some possibilities for performing this intelligent automatic selection include clustering or selecting the $k$-nearest-neighbors based on meta-features that describe these data sets, i.e., selecting those data sets from the list that have largest similarity according to a given similarity measure (e.g., Euclidean distance, Mahalanobis distance, etc.). In such an approach, the difficulty lies in choosing a set of meta-features that characterize the data sets in such a way that data sets with similar meta-features require similar design components of a decision-tree algorithm. This is, of course, not trivial, and an open problem in the meta-learning research field (Brazdil et al., 2009). 


\subsubsection{Parameter-Free Evolutionary Search}

One of the main challenges when dealing with evolutionary algorithms is the large amount of parameters one needs to set prior to its execution. In this thesis, we "avoided" this problem by consistently employing typical parameter values found in the literature of evolutionary algorithms for decision-tree induction. Nevertheless, we still tried to optimise the parameter $p$ in HEAD-DT, which controls the probabilities of crossover, mutation, and reproduction during evolution.

A challenging research effort would be the design of self-adaptive evolutionary parameters, which dynamically detect when their values need to change for the sake of improving the EA's performance. Research in that direction includes the work of Kramer (2008), which proposes self-adaptive crossover points, or the several studies on EAs for tuning EAs (Eiben and Smit, 2011).

\subsubsection{Solving the Meta-Overfitting Problem}

The meta-overfitting problem presented in Section 6.2.3 offers a good opportunity for future work. Recall that meta-overfitting occurs when we want to generate a good 'all around" algorithm, i.e., an algorithm that is robust across a set of different data sets. Some alternatives we envision for solving (or alleviating) this problem are:

- Increase the number of data sets in the meta-training set. Since the goal is to generate an "all-around" algorithm that performs well regardless of any particular data set characteristic, it is expected that feeding HEAD-DT with a larger meta-training set would increase the chances of achieving this goal. The disadvantage of this approach is that HEAD-DT becomes increasingly slower with each new data set that is added to the meta-training set.

\section{- Build a replacing mechanism during the evolutionary process that dynamically updates} the data sets in the meta-training set. By feeding HEAD-DT with different data sets per generation, we could guide the evolution towards robust "all-around" algorithms, avoiding the extra computational time spent in the previous solution. The problem with this strategy is that HEAD-DT would most likely provide an algorithm that performs well in the meta-training set used in the last generation of the evolution. This could be fixed by storing the best algorithm in each generation and then executing a single generation with these algorithms in the population, and with a full meta-training set (all data sets used in all generations).

- Build a meta-training set with diversity. A good "all-around" algorithm should perform reasonably well in all kinds of scenarios, and thus a possible solution is to feed HEAD-DT with data sets that cover a minimum level of diversity in terms of structural characteristics, that in turn represent a particular scenario. In practice, the problem with this approach is to identify the characteristics that really influence in predictive performance. As previously 
discussed, this is an open problem and a research area by itself, known as meta-learning, and we do believe that a full new thesis could be explored in this particular theme.

\subsubsection{Ensemble of Automatically-Designed Algorithms}

In this thesis, the strategy for automatically designing decision-tree induction algorithms aimed at the generation of effective algorithms, which were capable of outperforming other traditional manually-designed decision-tree algorithms. Another promising research direction would be to automatically design decision-tree algorithms to be used in an ensemble of classifiers. In this case, each individual would be an ensemble of automatically-designed algorithms, and a multi-objective fitness function could be employed to account for both the ensemble's predictive performance in a meta-training set and its diversity regarding each automatically-designed algorithm. For measuring diversity, one could think of a measure that would take into account the number of distinct correctly-classified instances between two different algorithms.

\subsubsection{Grammar-based Genetic Programming}

Finally, a natural extension of HEAD-DT would be regarding its search mechanism: instead of relying on a GA-like evolutionary algorithm, more sophisticated EAs such as standard grammar-based genetic programming (GGP) or grammatical evolution (GE) could be employed to evolve candidate decision trees. The latter seems to be an interesting research direction, since it is nowadays one of the most widely applied genetic programming methods (Mckay et al., 2010).

\subsection{Publications}

Throughout the research carried out during my $\mathrm{PhD}$, I have published conference and journal papers. Some of them are directly related to this thesis, whereas others are only indirectly-related to the main topic presented here. I present next the list of papers organised by themes.

[Decision-tree algorithms] — the following papers refer to decision-tree induction algorithms I developed during my $\mathrm{PhD}$. Basically, I developed a new bottom-up decision-tree induction algorithm called BUTIA, later generalised to a framework called BUTIF; I also developed a hybrid decision-tree/clustering approach called Clus-DTI, and helped in the development of a beam-search based decision-tree algorithm:

\section{Conference papers:}

- Barros, R. C.; Cerri, R.; Jaskowiak, P. A.; De Carvalho, A. C. P. L. F. A Bottom-Up Oblique Decision Tree Induction Algorithm. : 11th International Conference on Intelligent Systems Design and Applications, 450-456, 2011. 
- Barros, R. C.; De Carvalho, A. C. P. L. F.; Basgalupp, M. P.; Quiles, M. G. A Clustering-based Decision Tree Induction Algorithm. : 11th International Conference on Intelligent Systems Design and Applications, 543-550, 2011.

- Barros, R. C.; De Carvalho, A. C. P. L. F.; Basgalupp, M. P.; Quiles, M. G. Um Algoritmo de Indução de Árvores de Decisão baseado em Agrupamento. : VIII Encontro Nacional de Inteligência Artificial, 2011.

\section{Journal papers:}

- Barros, R. C.; Jaskowiak, P. A.; Cerri, R.; De Carvalho, A. C. P. L. F. A Framework for Bottom-Up Induction of Decision Trees. Neurocomputing, in press, 2014.

- Barros, R. C.; De Carvalho, A. C. P. L. F.; Basgalupp, M. P.; Quiles, M. G. Clus-DTI: Improving Decision-Tree Classification with a Clustering-based Decision-Tree Induction Algorithm. Journal of the Brazilian Computer Society, 18:4, 351-362, 2012.

\section{Book chapters:}

- Basgalupp, M. P.; Barros, R. C.; De Carvalho, A. C. P. L. F.; Freitas, A. A. A Beam-Search based Decision-Tree Induction Algorithm. : Machine Learning Algorithms for Problem Solving in Computational Applications: Intelligent Techniques, IGI-Global, 2011.

[Evolutionary algorithms to evolve decision trees] - the following papers refer to evolutionary algorithms I developed (or co-developed) to evolve decision trees (either for classification or for regression); the survey presented in Chapter 4 was published in a journal paper and is also present in this category:

\section{Conference papers:}

- Basgalupp, M. P.; Barros, R. C.; Ruiz, D. D. Predicting Software Maintenance Effort through Evolutionary based Decision Trees. :27th Annual ACM Symposium on Applied Computing, 1209-1214, 2012.

\section{Journal papers:}

- Barros, R. C.; Ruiz, D. D.; BAsgalupp, M. P. Evolutionary Model Trees for Handling Continuous Classes in Machine Learning. Information Sciences, 181, 954-971, 2011.

- Barros, R. C.; Basgalupp, M. P.; de Carvalho, A. C. P. L. F.; Freitas, A. A. A Survey of Evolutionary Algorithms for Decision-Tree Induction. IEEE Transactions on Systems, Man, and Cybernetics, Part C: Applications and Reviews, 42:3, 291-312, 2012. 
- Basgalupp, M. P.; Barros, R. C.; de Carvalho, A. C. P. L. F.; Freitas, A. A. Evolving Decision Trees with Beam Search-based Initialization and Lexicographic Multi-Objective Evaluation. Information Sciences, in press, 2013.

[Automatic design of decision-tree algorithms] - the following papers refer to the main contribution of this thesis, which is to automatically design decision-tree induction algorithms:

\section{Conference papers:}

- Barros, R. C.; Basgalupp, M. P.; de Carvalho, A. C. P. L. F.; Freitas, A. A. Towards the Automatic Design of Decision-Tree Induction Algorithms. : 13th Annual Conference Companion on Genetic and Evolutionary Computation (GECCO 2011), 567-574, 2011.

- Barros, R. C.; Basgalupp, M. P.; de Carvalho, A. C. P. L. F.; Freitas, A. A. A Hyper-Heuristic Evolutionary Algorithm for Automatically Designing Decision-Tree Algorithms. : 14th Annual Genetic and Evolutionary Computation Conference (GECCO 2012), 1237-1244, 2012.

- Basgalupp, M. P.; Barros, R. C.; Da Silva, T. S.; de Carvalho, A. C. P. L. F. Software Effort Prediction: A Hyper-Heuristic Decision-Tree based Approach. : 28th Annual ACM Symposium on Applied Computing, 1109-1116, 2013.

\section{Journal papers:}

- Barros, R. C.; Winck, A. T.; Machado, K. S.; Basgalupp, M. P.; De Carvalho, A. C. P. L. F.; Ruiz, D. D.; De SouzA, O. N. Automatic Design of Decision-Tree Induction Algorithms Tailored to Flexible-Receptor Docking Data. BMC Bioinformatics, 13, 310, 2012.

- Barros, R. C.; Basgalupp, M. P.; De Carvalho, A. C. P. L. F.; Freitas, A. A. Automatic Design of Decision-Tree algorithms with Evolutionary Algorithms. Evolutionary Computation, 21:4, 2013.

- Barros, R. C.; Basgalupp, M. P.; Freitas, A. A.; de Carvalho, A. C. P. L. F. Evolutionary Design of Decision-Tree Algorithms Tailored to Microarray Gene Expression Data Sets. IEEE Transactions on Evolutionary Computation, under review, 2014.

[Hierarchical multi-label classification] — the following papers refer to the area of hierarchical multi-label classification, gently presented to me by my friend and research partner Ricardo Cerri:

\section{Conference papers:}


- Cerri, R.; Barros, R. C.; De Carvalho, A. C. P. L. F. Hierarchical Multi-Label Classification for Protein Function Prediction: A Local Approach based on Neural Networks. : 11th International Conference on Intelligent Systems Design and Applications, 337-343, 2011.

- Cerri, R.; Barros, R. C.; De Carvalho, A. C. P. L. F. Redes Neurais Artificiais Aplicadas a Problemas de Classificação Hierárquica Multirrótulo. : X Congresso Brasileiro de Inteligência Computacional, 2011.

- Cerri, R.; Barros, R. C.; De Carvalho, A. C. P. L. F. A Genetic Algorithm for Hierarchical Multi-Label Classification. :27th Annual ACM Symposium on Applied Computing, 250-255, 2012.

- Barros, R. C.; Cerri, R.; Freitas, A. A.; de Carvalho, A. C. P. L. F. Probabilistic Clustering for Hierarchical Multi-Label Classification of Protein Functions. : Machine Learning and Knowledge Discovery in Databases (ECML/PKDD 2013), Springer Berlin Heidelberg, 2013.

- Cerri, R.; Barros, R. C.; De Carvalho, A. C. P. L. F. Neural Networks for Hierarchical Classification of G-Protein Coupled Receptors. : 2nd Brazilian Conference on Intelligent Systems, 2013.

- Cerri, R.; Barros, R. C.; de Carvalho, A. C. P. L. F.; Freitas, A. A. A Grammatical Evolution Algorithm for Generation of Hierarchical Multi-Label Classification Rules. : IEEE Congress on Evolutionary Computation, 454-461, 2013.

\section{Journal papers:}

- Cerri, R.; Barros, R. C.; De Carvalho, A. C. P. L. F. Hierarchical Multi-Label Classification using Local Neural Networks. Journal of Computer and System Sciences, 80:1, 39-56, 2014.

- Cerri, R.; Barros, R. C.; de Carvalho, A. C. P. L. F.; Jin, Y. Neural Networks for Hierarchical Multi-Label Classification. IEEE Transactions on Neural Networks and Learning Systems, under review, 2014.

[Other themes] - the following papers refer to work I developed and co-developed in the areas of oscillatory correlation for semi-supervised classification, data stream clustering, bottom-up safe semi-supervised SVMs, KDD in oral health, and software effort prediction with grammatical evolution:

\section{Conference papers:}


- Quiles, M. G.; Basgalupp, M. P.; Barros, R. C. Um Modelo de Correlação Oscilatória para Classificação Semi-Supervisionada. :IV Encontro Nacional de Inteligência Artificial, 2012.

- Blomberg, L. C.; Barros, R. C.; Figueiredo, J. A.; Ruiz D. D. Aplying a KDD Process in a Oral Public Health Unit at Rio Grande do Sul, Brazil. : IADIS International Conference, Lisboa, Portugal, 2013.

- Barros, R. C.; Basgalupp, M. P.; Cerri, R.; Da Silva, T. S.; De Carvalho, A. C. P. L. F. A Grammatical Evolution Approach for Software Effort Estimation. : 15th Annual Genetic and Evolutionary Computation Conference (GECCO 2013), 1413-1420, 2013.

\section{Journal papers:}

- Quiles, M. G.; Basgalupp, M. P.; Barros, R. C. An Oscillatory Correlation Model for Semi-Supervised Classification. Learning and Nonlinear Models, 11, 3-10, 2013.

- Silva, J. A.; Faria, E. R.; Barros, R. C.; Hruschka, E. R.; de Carvalho, A. C. P. L. F.; Gama, J. P. Data Stream Clustering: A Survey. ACM Computing Surveys, 46:1, 2014.

- Covões, T. F.; Barros, R. C.; Silva, T. S.; Hruschka, E. R.; de Carvalho, A. C. P. L. F. Hierarchical Bottom-Up Safe Semi-Supervised Support Vector Machines for Multi-Class Transductive Learning. Journal of Information and Data Management, 4:3, 357-372, 2013.

[Awards] — the following papers received the "best paper award" in their respective events:

- One of the 3 best papers at ENIA 2011: Barros, R.C.; De Carvalho, A.C.P.L.F.; BasgalupP, M.P.; Quiles, M.G. Um Algoritmo de Indução de Árvores de Decisão baseado em Agrupamento. : VIII Encontro Nacional de Inteligência Artificial, 2011.

- Best paper in the IGEC+S*S+SBSE tracks at GECCO 2012: BARros, R. C.; Basgalupp, M. P.; de Carvalho, A. C. P. L. F.; Freitas, A. A. A Hyper-Heuristic Evolutionary Algorithm for Automatically Designing Decision-Tree Algorithms. : 14th Annual Genetic and Evolutionary Computation Conference (GECCO 2012), 1237-1244, 2012.

- Best student paper at IEEE CEC 2013: Cerri, R.; Barros, R. C.; De Carvalho, A. C. P. L. F.; Freitas, A. A. A Grammatical Evolution Algorithm for Generation of Hierarchical Multi-Label Classification Rules. : IEEE Congress on Evolutionary Computation, 454-461, 2013. 
- José Mauro Castilho Award for best full paper at SBBD 2013: Covões, T. F.; Barros, R. C.; Silva, T. S.; Hruschka, E. R.; De Carvalho, A. C. P. L. F. Hierarchical Bottom-Up Safe Semi-Supervised Support Vector Machines for Multi-Class Transductive Learning. Journal of Information and Data Management, 4:3, 357-372, 2013. 

Agresti, A. Categorical Data Analysis. Wiley Series in Probability and Statistics, 2nd edn.. Wiley-Interscience, 2002. (Cited on pages 23 and 27.)

Aitkenhead, M. J. A co-evolving decision tree classification method. Expert Systems with Applications, vol. 34, no. 1, pp. 18-25, 2008. (Cited on pages xi, 58, 61, and 62.)

AlPAydin, E. Introduction to Machine Learning. 2nd edn.. MIT Press, 2010. (Cited on pages 10,26 , and 27.)

Angeline, P. J. Subtree Crossover: Building block engine or Macromutation. In: Second Annual Conference on Genetic Programming, pp. 9-17, 1997. (Cited on page 52.)

BACK, T. Selective pressure in evolutionary algorithms: a characterization of selection mechanisms. In: IEEE Conference on Evolutionary Computation (CEC 1994), pp. 57-62, 1994. (Cited on page 50.)

BAIM, P. W. A method for attribute selection in inductive learning systems. IEEE Transactions on Pattern Analysis and Machine Intelligence, vol. 10, no. 6, pp. 888-896, 1988. (Cited on pages 24 and 27.)

Bandar, Z.; Al-AtTar, H.; MCLean, D. Genetic algorithm based multiple decision tree induction. In: International Conference on Neural Information Processing, pp. 429-434, 1999. (Cited on pages 59, 62, 67, and 69.)

Banzhaf, W.; Francone, F. D.; Keller, R. E.; Nordin, P. Genetic programming: an introduction - On the automatic evolution of computer programs and its applications. San Francisco, CA, USA: Morgan Kaufmann Publishers Inc., 1998. (Cited on pages 48, 52, and 71.)

Barros, R. C.; Basgalupp, M. P.; de Carvalho, A. C. P. L. F.; Freitas, A. A. Towards the automatic design of decision tree induction algorithms. In: 13th Annual Conference 
Companion on Genetic and Evolutionary Computation (GECCO 2011), pp. 567-574, 2011a. (Cited on pages 90 and 110.)

Barros, R. C.; Basgalupp, M. P.; De Carvalho, A. C. P. L. F.; Freitas, A. A. A Survey of Evolutionary Algorithms for Decision-Tree Induction. IEEE Transactions on Systems, Man, and Cybernetics, Part C: Applications and Reviews, vol. 42, no. 3, pp. 291-312, 2012a. (Cited on pages 2 and 103.)

Barros, R. C.; Basgalupp, M. P.; Ruiz, D.; Carvalho, A. C. P. L. F.; Freitas, A. A. Evolutionary model tree induction. In: ACM Symposium on Applied Computing, pp. 1131-1137, 2010. (Cited on pages 59 and 76.)

Barros, R. C.; Cerri, R.; Jaskowiak, P. A.; de Carvalho, A. C. P. L. F. A Bottom-Up Oblique Decision Tree Induction Algorithm. In: 11th International Conference on Intelligent Systems Design and Applications, pp. 450-456, 2011b. (Cited on pages 1, 42, and 167.)

Barros, R. C.; Jaskowiak, P. A.; Cerri, R.; De Carvalho, A. C. P. L. F. A Framework for Bottom-Up Induction of Decision Trees. Neurocomputing, vol. in press, 2013. (Cited on pages 42 and 167.)

Barros, R. C.; Ruiz, D. D.; Basgalupp, M. P. Evolutionary model trees for handling continuous classes in machine learning. Information Sciences, vol. 181, pp. 954-971, 2011c. (Cited on pages 59, 76, and 90.)

Barros, R. C.; Winck, A. T.; Machado, K. S.; Basgalupp, M. P.; De Carvalho, A. C. P. L. F.; Ruiz, D. D.; DE SouzA, O. S. Automatic design of decision-tree induction algorithms tailored to flexible-receptor docking data. BMC Bioinformatics, vol. 13, 2012b. (Cited on page 140.)

Basgalupp, M.; Barros, R. C.; Carvalho, A. C. P. L. F.; Freitas, A. A.; Ruiz, D. LEGAL-Tree: a lexicographic multi-objective genetic algorithm for decision tree induction. In: ACM Symposium on Applied Computing, pp. 1085-1090, 2009a. (Cited on pages 59, 61, 65, $66,67,69$, and 70.)

Basgalupp, M. P.; Barros, R. C.; de Carvalho, A. C. P. L. F.; Freitas, A. A. A beam-search based decision-tree induction algorithm. In: Machine Learning Algorithms for Problem Solving in Computational Applications: Intelligent Techniques, IGI-Global, 2011. (Cited on pages 42 and 167.)

Basgalupp, M. P.; Barros, R. C.; DA Silva, T. S.; De Carvalho, A. C. P. L. F. Software effort prediction: a hyper-heuristic decision-tree based approach. In: 28th Annual ACM Symposium on Applied Computing, pp. 1109-1116, 2013. (Cited on page 140.) 
Basgalupp, M. P.; De Carvalho, A. C. P. L. F.; Barros, R. C.; Ruiz, D. D.; Freitas, A. A. Lexicographic multi-objective evolutionary induction of decision trees. International Journal of Bio-inspired Computation, vol. 1, no. 1/2, pp. 105-117, 2009b. (Cited on pages 47, $59,62,66,67,69$, and 70.)

BennetT, K. Global tree optimization: A non-greedy decision tree algorithm. Computing Science and Statistics, pp. 156-160, 1994. (Cited on page 43.)

Bennett, K.; Mangasarian, O. Robust Linear Programming Discrimination of Two Linearly Inseparable Sets. Optimization Methods and Software, vol. 1, pp. 23-34, 1992. (Cited on page 32.)

Bennett, K.; Mangasarian, O. Multicategory discrimination via linear programming. Optimization Methods and Software, vol. 2, pp. 29-39, 1994. (Cited on pages 2 and 32.)

Blickle, T.; THIElE, L. A comparison of selection schemes used in evolutionary algorithms. Tech. Rep., Swiss Federal Institute of Technology, 1995. (Cited on page 50.)

Blickle, T.; Thiele, L. A comparison of selection schemes used in evolutionary algorithms. Evolutionary Computation, vol. 4, pp. 361-394, 1996. (Cited on page 70.)

BobrowsKi, L. Piecewise-linear classifiers, formal neurons and separability of the learning sets. In: International Conference on Pattern Recognition, pp. 224 -228, 1996. (Cited on page 72.)

BobrowsKi, L.; Kretowski, M. Induction of Multivariate Decision Trees by Using Dipolar Criteria. In: European Conference on Principles of Data Mining and Knowledge Discovery, pp. 331-336, 2000. (Cited on pages 31 and 32.)

Bонм, W.; GeYer-Schulz, A. Exact uniform initialization for genetic programming. In: Foundations of Genetic Algorithms IV, Morgan Kaufmann, pp. 379-407, 1996. (Cited on page 49.)

Bot, M. C.; LAngdon, W. B. Application of genetic programming to induction of linear classification trees. In: Genetic Programming, vol. 1802 of Lecture Notes in Computer Science, Springer Berlin / Heidelberg, pp. 247-258, 2004. (Cited on page 71.)

Bot, M. C. J.; BoelelaAn, D.; LANGdon, W. B. Improving induction of linear classification trees with genetic programming. In: Genetic and Evolutionary Computation Conference (GECCO 2000), pp. 403-410, 2000. (Cited on page 71.)

Bot, M. C. J.; LAngdon, W. B. Application of genetic programming to induction of linear classification trees. In: European Conference on Genetic Programming, pp. 247-258, 2000. (Cited on page 71.) 
Bratu, C.; Savin, C.; Potolea, R. A hybrid algorithm for medical diagnosis. In: International Conference on Computer as a Tool (EUROCON), pp. 668 -673, 2007. (Cited on pages 56 and 83.)

Brazdil, P.; Giraud-Carrier, C. G.; Soares, C.; Vilalta, R. Metalearning applications to data mining. Cognitive Technologies. Springer, I-X, 1-176 pp., 2009. (Cited on page 168.)

Breiman, L. Random forests. Machine Learning, vol. 45, no. 1, pp. 5-32, 2001. (Cited on page 2.)

Breiman, L.; Friedman, J. H.; Olshen, R. A.; Stone, C. J. Classification and regression trees. Wadsworth, 1984. (Cited on pages 1, 5, 18, 24, 25, 27, 32, 33, 36, 39, 40, 44, 55, 74, 79, 92, 94, 95, 96, 106, and 165.)

Breslow, L.; Aha, D. Simplifying decision trees: A survey. Knowledge Engineering Review, vol. 12, no. 01, pp. 1-40, 1997. (Cited on pages 2, 34, and 39.)

Brodley, C. E.; Utgoff, P. E. Multivariate versus univariate decision trees. Tech. Rep., Department of Computer Science, University of Massachusetts at Amherst, 1992. (Cited on pages 28 and 32.)

Brodley, C. E.; Utgoff, P. E. Multivariate Decision Trees. Machine Learning, vol. 19, no. 1, pp. 45-77, 1995. (Cited on pages xi and 28.)

BuJA, A.; LEE, Y.-S. Data mining criteria for tree-based regression and classification. In: ACM SIGKDD International Conference on Knowledge Discovery and Data Mining, pp. 27-36, 2001. (Cited on pages 25 and 27.)

Buntine, W. A theory of learning classification rules. Ph.D. thesis, University of Technology, Sidney, 1992a. (Cited on page 39.)

Buntine, W. Learning classification trees. Statistics and Computing, vol. 2, pp. 63-73, 1992b. (Cited on page 42.)

Burgess, C. J.; Lefley, M. Can genetic programming improve software effort estimation? A comparative evaluation. Information and Software Technology, vol. 43, no. 14, pp. 863 - 873, 2001. (Cited on page 56.)

Burke, E.; Gustafson, S.; Kendall, G. Ramped half-n-half initialisation bias in GP. In: Genetic and Evolutionary Computation Conference (GECCO 2003), pp. 1800-1801, 2003a. (Cited on page 49.)

Burke, E.; Petrovic, S. Recent research directions in automated timetabling. European Journal of Operational Research, vol. 140, no. 2, pp. 266-280, 2002. (Cited on page 90.) 
Burke, E. K.; Hyde, M.; Kendall, G.; OchoA, G.; ÖZcan, E.; QU, R. A survey of hyper-heuristics. Tech. Rep. Computer Science Technical Report No. NOTTCS-TR-SUB-0906241418-2747, School of Computer Science and Information Technology, University of Nottingham, 2009. (Cited on page 52.)

Burke, E. K.; Hyde, M.; Kendall, G.; Ochoa, G.; Ozcan, E.; Woodward, J. R. A classification of hyper-heuristics approaches. In: Gendreau, M.; Potvin, J.-Y., eds. Handbook of Metaheuristics, vol. 57 of International Series in Operations Research \& Management Science, 2nd edn., chap. 15, Springer, pp. 449-468, 2010. (Cited on page 90.)

Burke, E. K.; Kendall, G.; Soubeiga, E. A Tabu-Search Hyperheuristic for Timetabling and Rostering. Journal of Heuristics, vol. 9, no. 6, pp. 451-470, 2003b. (Cited on pages 53 and 90.)

Burke, E. K.; McCollum, B.; Meisels, A.; Petrovic, S.; Qu, R. A graph-based hyper-heuristic for educational timetabling problems. European Journal of Operational Research, vol. 176, no. 1, pp. 177 - 192, 2007. (Cited on page 53.)

Burke, E. K.; Petrovic, S.; Qu, R. Case-based heuristic selection for timetabling problems. Journal of Scheduling, vol. 9, no. 2, pp. 115-132, 2006. (Cited on page 53.)

CANTU-PAZ, E.; KAMATH, C. Using evolutionary algorithms to induce oblique decision trees. In: Genetic and Evolutionary Computation Conference (GECCO 2000), pp. 1053-1060, 2000. (Cited on pages 79 and 83.)

CAntu-Paz, E.; Kamath, C. Inducing oblique decision trees with evolutionary algorithms. IEEE Transactions on Evolutionary Computation, vol. 7, no. 1, pp. 54 - 68, 2003. (Cited on page 79.)

Cantu-Paz, E.; Kamath, C.; Kamath, R. Combining Evolutionary Algorithms With Oblique Decision Trees to Detect Bent-Double Galaxies. In: International Symposium on Optical Science and Technology, pp. 63-71, 2000. (Cited on page 83.)

CASEy, R.; Nagy, G. Decision tree design using a probabilistic model. IEEE Transactions on Information Theory, vol. 30, no. 1, pp. 93-99, 1984. (Cited on pages 15 and 27.)

Cestnik, B.; BRAtKo, I. On estimating probabilities in tree pruning. In: Machine Learning - EWSL-91, vol. 482 of Lecture Notes in Computer Science, Springer Berlin/Heidelberg, pp. 138-150, 1991. (Cited on pages 35, 36, 95, and 96.)

CHA, S.-H.; TAPPERT, C. A genetic algorithm for constructing compact binary decision trees. Journal of Pattern Recognition Research, vol. 1, pp. 1-13, 2009. (Cited on pages 61, 67, and 68.)

CHAI, B.-B. Binary linear decision tree with genetic algorithm. In: International Conference on Pattern Recognition (ICPR 1996), pp. 530-, 1996. (Cited on pages 56 and 77.) 
Chai, B.-B.; Huang, T.; Zhuang, X.; Zhao, Y.; Sklansky, J. Piecewise linear classifiers using binary tree structure and genetic algorithm. Pattern Recognition, vol. 29, no. 11, pp. 1905 - 1917, 1996. (Cited on pages 56 and 77.)

Chandra, B.; Kothari, R.; Paul, P. A new node splitting measure for decision tree construction. Pattern Recognition, vol. 43, no. 8, pp. 2725-2731, 2010. (Cited on pages 23, 24, 27, 92, and 135.)

Chandra, B.; Varghese, P. P. Moving towards efficient decision tree construction. Information Sciences, vol. 179, no. 8, pp. 1059-1069, 2009. (Cited on pages 23, 27, 92, and 135.)

Chellapilla, K. Evolving computer programs without subtree crossover. IEEE Transactions on Evolutionary Computation, vol. 1, no. 3, pp. 209-216, 1997. (Cited on page 49.)

Chen, J.; WAng, X.; Zhai, J. Pruning decision tree using genetic algorithms. In: International Conference on Artificial Intelligence and Computational Intelligence (AICI 2009), pp. 244-248, 2009. (Cited on pages 56 and 81.)

Ching, J.; Wong, A.; ChAn, K. Class-dependent discretization for inductive learning from continuous and mixed-mode data. IEEE Transactions on Pattern Analysis and Machine Intelligence, vol. 17, no. 7, pp. 641-651, 1995. (Cited on pages 14, 27, and 92.)

CHIS, M. Evolutionary decision trees and software metrics for module defects identification. In: World Academy of Science, Engineering and Technology (WASET), pp. 273-277, 2008. (Cited on pages 83 and 85.)

CHou, P. Optimal partitioning for classification and regression trees. IEEE Transactions on Pattern Analysis and Machine Intelligence, vol. 13, no. 4, pp. 340-354, 1991. (Cited on page 42.)

Clark, P.; Niblett, T. The CN2 induction algorithm. Machine Learning, vol. 3, no. 4, pp. 261-283, 1989. (Cited on pages 40 and 94.)

Coello, C. A Comprehensive Survey of Evolutionary-Based Multiobjective Optimization Techniques. Knowledge and Information Systems, vol. 1, no. 3, pp. 129-156, 1999. (Cited on page 50.)

Coello, C. A. C.; Lamont, G. B.; Veldhuizen, D. A. V. Evolutionary algorithms for solving multi-objective problems. Genetic and Evolutionary Computation. Secaucus, NJ, USA: Springer-Verlag New York, Inc., 2006. (Cited on page 50.)

Coppersmith, D.; Hong, S. J.; Hosking, J. R. M. Partitioning nominal attributes in decision trees. Data Mining and Knowledge Discovery, vol. 3, pp. 197-217, 1999. (Cited on page 18.) 
Cowling, P.; Kendall, G.; Soubeiga, E. A hyperheuristic approach to scheduling a sales summit. In: Burke, E.; Erben, W., eds. Practice and Theory of Automated Timetabling III, vol. 2079 of Lecture Notes in Computer Science, Springer Berlin Heidelberg, pp. 176-190, 2001a. (Cited on page 3.)

Cowling, P. I.; Kendall, G.; Soubeiga, E. A hyperheuristic approach to scheduling a sales summit. In: Third International Conference on Practice and Theory of Automated Timetabling, London, UK, UK: Springer-Verlag, pp. 176-190, 2001b. (Cited on page 53.)

CuOng, T.; PHAm, T. Analysis of cardiac imaging data using decision tree based parallel genetic programming. In: 6th International Symposium on Image and Signal Processing and Analysis, pp. $317-320,2009$. (Cited on pages 56, 83, and 84.)

De Mántaras, R. L. A distance-based attribute selection measure for decision tree induction. Machine Learning, vol. 6, no. 1, pp. 81-92, 1991. (Cited on pages 19, 27, 55, and 92.)

DE'ATH, G. Multivariate Regression Trees: A New Technique for Modeling Species-Environment Relationships. Ecology, vol. 83, no. 4, pp. 1105-1117, 2002. (Cited on page 32.)

Delibasic, B.; Jovanovic, M.; Vukicevic, M.; Suknovic, M.; Obradovic, Z. Component-based decision trees for classification. Intelligent Data Analysis, vol. 15, pp. 1-38, 2011. (Cited on page 103.)

DeLisle, R. K.; DiXON, S. L. Induction of decision trees via evolutionary programming. Journal of Chemical Information and Computer Sciences, vol. 44, no. 3, pp. 862-870, 2004. (Cited on pages 61, 64, 66, 67, and 69.)

DEMŠAR, J. Statistical comparisons of classifiers over multiple data sets. Journal of Machine Learning Research, vol. 7, pp. 1-30, 2006. (Cited on pages 107, 120, 129, and 136.)

Devroye, L.; Györfi, L.; Lugosi, G. A Probabilistic Theory of Pattern Recognition. Springer, 1996. (Cited on page 17.)

Dong, M.; Kothari, R. Look-ahead based fuzzy decision tree induction. IEEE Transactions on Fuzzy Systems, vol. 9, no. 3, pp. 461-468, 2001. (Cited on page 42.)

Draper, B.; Brodley, C. Goal-directed classification using linear machine decision trees. IEEE Transactions on Pattern Analysis and Machine Intelligence, vol. 16, no. 9, pp. 888-893, 1994. (Cited on page 28.)

Eggermont, J.; KoK, J.; Kosters, W. Genetic programming for data classification: Refining the search space. In: 15th Belgium/Netherlands Conference on Artificial Intelligence (BNAIC'03), pp. 123-130, 2003. (Cited on pages 61, 65, 66, 67, 69, and 70.) 
Eggermont, J.; KoK, J. N.; Kosters, W. A. Detecting and pruning introns for faster decision tree evolution. In: Yao, X.; Burke, E.; Lozano, J. A.; Smith, J.; Merelo-Guervos, J. J.; Bullinaria, J. A.; Rowe, J.; Tino, P.; Kaban, A.; Schwefel, H.-P., eds. Parallel Problem Solving from Nature - PPSN VIII, vol. 3242 of Lecture Notes in Computer Science, Springer Berlin / Heidelberg, pp. 1071-1080, 2004a. (Cited on pages 61, 66, 67, 69, and 70.)

Eggermont, J.; KoK, J. N.; Kosters, W. A. Genetic programming for data classification: partitioning the search space. In: SAC '04: 2004 ACM symposium on Applied computing, New York, NY, USA: ACM, pp. 1001-1005, 2004b. (Cited on pages 61, 65, 66, 67, 69, and 70.)

Eiben, A. E.; SMit, S. K. Parameter tuning for configuring and analyzing evolutionary algorithms. Swarm and Evolutionary Computation, vol. 1, no. 1, pp. 19-31, 2011. (Cited on page 169.)

Eiben, A. E.; Smith, J. E. Introduction to evolutionary computing. Springer Verlag, 2003. (Cited on page 48.)

Eiben, A. E.; Smith, J. E. Introduction to Evolutionary Computing (Natural Computing Series). Springer, 2008. (Cited on page 2.)

Esmeir, S.; Markovitch, S. Anytime Learning of Decision Trees. Journal of Machine Learning Research, vol. 8, pp. 891-933, 2007. (Cited on page 43.)

Espejo, P. G.; Ventura, S.; Herrera, F. A survey on the application of genetic programming to classification. IEEE Transactions on Systems, Man, and Cybernetics, Part C: Applications and Reviews, vol. 40, no. 2, pp. 121-144, 2010. (Cited on pages 56, 68, and 86.)

Esposito, F.; Malerba, D.; Semeraro, G. A further study of pruning methods in decision tree induction. In: Fifth International Workshop on Artificial Intelligence and Statistics, pp. 211-218, 1995a. (Cited on page 37.)

Esposito, F.; Malerba, D.; Semeraro, G. Simplifying Decision Trees by Pruning and Grafting: New Results (Extended Abstract). In: 8th European Conference on Machine Learning, ECML '95, London, UK: Springer-Verlag, pp. 287-290, 1995b (ECML '95, ). (Cited on page 37.$)$

Esposito, F.; Malerba, D.; Semeraro, G. A Comparative Analysis of Methods for Pruning Decision Trees. IEEE Transactions on Pattern Analysis and Machine Intelligence, vol. 19, no. 5, pp. 476-491, 1997. (Cited on pages 2, 32, 33, 34, 35, 37, 39, and 96.)

Estrada-Gil, J. K.; Fernandez-Lopez, J. C.; HernandeZ-Lemus, E.; Silva-Zolezzi, I.; Hidalgo-Miranda, A.; Jimenez-Sanchez, G.; Vallejo-Clemente, E. E. GPDTI: A Genetic Programming Decision Tree Induction method to find epistatic effects in common 
complex diseases. Bioinformatics, vol. 23, no. 13, pp. 167-174, 2007. (Cited on pages 83 and 84.)

FAN, G.; GRAY, J. Regression tree analysis using TARGET. Journal of Computational and Graphical Statistics, vol. 14, no. 1, pp. 206-218, 2005. (Cited on page 73.)

FAwCETt, T. An introduction to ROC analysis. Pattern Recognition Letters, vol. 27, no. 8, pp. 861-874, 2006. (Cited on page 145.)

FAYYAD, U.; IRANI, K. The attribute selection problem in decision tree generation. In: National Conference on Artificial Intelligence, pp. 104-110, 1992. (Cited on pages 19, 20, 27, and 92.)

FAYYAD, U. M. On the induction of decision trees for multiple concept learning. Ph.D. thesis, University of Michigan, Ann Arbor, MI, USA, 1992. (Cited on page 65.)

Ferri, C.; Hernández-Orallo, J.; Modroiu, R. An Experimental Comparison of Performance Measures for Classification. Pattern Recognition Letters, vol. 30, no. 1, pp. 27-38, 2009. (Cited on page 144.)

Fisher, H.; ThOMPson, G. L. Probabilistic learning combinations of local job-shop scheduling rules. In: Muth, J. F.; Thompson, G. L., eds. Industrial Scheduling, Prentice Hall, pp. 225-251, 1963. (Cited on page 53.)

Folino, G.; Pizzuti, C.; Spezzano, G. A cellular genetic programming approach to classification. In: Genetic and Evolutionary Computation Conference (GECCO99), Morgan Kaufmann, pp. 1015-1020, 1999. (Cited on pages 66 and 67.)

Folino, G.; Pizzuti, C.; Spezzano, G. Genetic programming and simulated annealing: A hybrid method to evolve decision trees. In: European Conference on Genetic Programming, London, UK: Springer-Verlag, pp. 294-303, 2000. (Cited on pages 61, 62, 66, 67, 69, and 70.)

Folino, G.; Pizzuti, C.; Spezzano, G. Parallel genetic programming for decision tree induction. In: 13th IEEE International Conference on Tools with Artificial Intelligence (ICTAI '01), Washington, DC, USA: IEEE Computer Society, p. 129, 2001. (Cited on pages 61, 62, 66, 67, 69, and 70.)

Folino, G.; Pizzuti, C.; Spezzano, G. Improving induction decision trees with parallel genetic programming. In: Euromicro Conference on Parallel, Distributed, and Network-Based Processing, Los Alamitos, CA, USA: IEEE Computer Society, pp. 1-7, 2002. (Cited on pages $61,62,66,67,69$, and 70 .)

FrAnK, A.; Asuncion, A. UCI machine learning repository. 2010.

Available at http: / / archive.ics.uci.edu/ml (Cited on pages 6, 23, 39, 53, 100, 106, 122, and 166.) 
FreItAS, A. A. Data mining and knowledge discovery with evolutionary algorithms. Secaucus, NJ, USA: Springer-Verlag New York, Inc., 2002. (Cited on pages xv, 2, 46, 54, 56, and 86.)

Freitas, A. A. A critical review of multi-objective optimization in data mining: a position paper. SIGKDD Explor. Newsl., vol. 6, no. 2, pp. 77-86, 2004. (Cited on pages 23, 62, 66, and 168.)

Freitas, A. A. A review of evolutionary algorithms for data mining. In: MaImon, O.; RoKACH, L., eds. Soft Computing for Knowledge Discovery and Data Mining, Springer US, pp. 79-111, 2008. (Cited on pages 2, 45, 50, and 62.)

Freitas, A. A.; Wieser, D. C.; Apweiler, R. On the importance of comprehensible classification models for protein function prediction. IEEE/ACM Transactions on Computational Biology and Bioinformatics, vol. 7, pp. 172-182, 2010. (Cited on pages 1 and 56.)

FRIEDMAN, J. H. A recursive partitioning decision rule for nonparametric classification. IEEE Transactions on Computers, vol. 100, no. 4, pp. 404-408, 1977. (Cited on pages 18, 27, 40, and 94.)

Fu, Z.; Golden, B.; Lele, S.; Raghavan, S.; Wasil, E. Genetically engineered decision trees: Population diversity produces smarter trees. Operations Research, vol. 51, no. 6, pp. 894-907, 2003a. (Cited on pages 59, 62, 66, 67, 68, and 69.)

Fu, Z.; Golden, B. L.; Lele, S.; Raghavan, S.; Wasil, E. Diversification for better classification trees. Computers \& Operations Research, vol. 33, no. 11, pp. 3185-3202, 2006. (Cited on pages 59, 61, 63, 66, 67, 68, and 69.)

Fu, Z.; Golden, B. L.; Lele, S.; Raghavan, S.; Wasil, E. A. A genetic algorithm-based approach for building accurate decision trees. INFORMS Journal on Computing, vol. 15, no. 1, pp. 3-22, 2003b. (Cited on pages 59, 62, 66, 67, 68, 69, and 83.)

FU, Z.; MAE, F. A computational study of using genetic algorithms to develop intelligent decision trees. In: IEEE Congress on Evolutionary Computation (CEC 2001), pp. 1382 -1387, 2001. (Cited on pages 59, 61, 62, 67, 68, and 69.)

Gagné, C.; PARIZEAu, M. Genericity in evolutionary computation software tools: Principles and case study. International Journal on Artificial Intelligence Tools, vol. 15, no. 2, pp. 173-194, 2006. (Cited on page 75.)

Garcia-AlmanzA, A.; TSAng, E. Simplifying decision trees learned by genetic programming. In: IEEE Congress on Evolutionary Computation (CEC 2006), pp. $2142-2148$, 2006. (Cited on pages 61, 65, 66, 69, and 70.) 
Garrido, P.; CAStro, C. Stable solving of cvrps using hyperheuristics. In: Proceedings of the 11th Annual conference on Genetic and evolutionary computation, GECCO '09, New York, NY, USA: ACM, pp. 255-262, 2009 (GECCO '09, ). (Cited on page 54.)

GARRIDO, P.; RIFF, M.-C. An evolutionary hyperheuristic to solve strip-packing problems. In: Proceedings of the 8th international conference on Intelligent data engineering and automated learning, IDEAL'07, Berlin, Heidelberg: Springer-Verlag, pp. 406-415, 2007 (IDEAL'07, ). (Cited on page 53.)

GARRIDO, P.; RifF, M. C. Dvrp: a hard dynamic combinatorial optimisation problem tackled by an evolutionary hyper-heuristic. Journal of Heuristics, vol. 16, no. 6, pp. 795-834, 2010. (Cited on page 54.)

GATHERCOLE, C. An investigation of supervised learning in genetic programming. Ph.D. thesis, University of Edinburgh, 1998. (Cited on page 71.)

Gelfand, S. B.; Ravishankar, C. S.; DelP, E. J. An iterative growing and pruning algorithm for classification tree design. In: IEEE International Conference on Systems, Man and Cybernetics, IEEE, pp. 818-823, 1989. (Cited on page 18.)

GILLO, M. W. MAID: A honeywell 600 program for an automatised survey analysis. Behavioral Science, vol. 17, pp. 251-252, 1972. (Cited on page 9.)

Gleser, M.; Collen, M. Towards automated medical decisions. Computers and Biomedical Research, vol. 5, no. 2, pp. 180-189, 1972. (Cited on pages 14, 27, and 92.)

GoldBerg, D. E. Genetic algorithms in search, optimization and machine learning. Boston, MA, USA: Addison-Wesley Longman Publishing Co., Inc., 1989. (Cited on page 71.)

GoldberG, D. E.; Deb, K. A comparative analysis of selection schemes used in genetic algorithms. In: RAWLINS, G. J. E., ed. Foundations of Genetic Algorithms, San Mateo, CA: Morgan Kaufmann, pp. 69-93, 1991. (Cited on page 50.)

Goodman, L. A.; Kruskal, W. H. Measures of association for cross classifications. Journal of the American Statistical Association, vol. 49, no. 268, pp. 732-764, 1954. (Cited on page 21.)

Gray, J.; FAn, G. Classification tree analysis using TARGET. Computational Statistics \& Data Analysis, vol. 52, pp. 1362-1372, 2008. (Cited on page 73.)

HAizhou, D.; ChOng, M. Study on constructing generalized decision tree by using dna coding genetic algorithm. In: 2009 International Conference on Web Information Systems and Mining (WISM '09), Washington, DC, USA: IEEE Computer Society, pp. 163-167, 2009. (Cited on pages 61,64 , and 67.) 
HANCOCK, T.; JiAng, T.; LI, M.; TROMP, J. Lower bounds on learning decision lists and trees. Information and Computation, vol. 126, no. 2, 1996. (Cited on page 11.)

Hanczar, B.; Hua, J.; Sima, C.; Weinstein, J.; Bittner, M.; Dougherty, E. R. Small-sample precision of ROC-related estimates. Bioinformatics, vol. 26, no. 6, pp. 822-830, 2010. (Cited on page 146.)

HAND, D. J. Measuring classifier performance: a coherent alternative to the area under the ROC curve. Machine Learning, vol. 77, no. 1, pp. 103-123, 2009. (Cited on page 146.)

Harik, G.; Cantú-Paz, E.; Goldberg, D. E.; Miller, B. L. The gambler's ruin problem, genetic algorithms, and the sizing of populations. Evolutionary Computation, vol. 7, no. 3, pp. 231-253, 1999. (Cited on page 79.)

Hartmann, C.; Varshney, P.; Mehrotra, K.; Gerberich, C. Application of information theory to the construction of efficient decision trees. IEEE Transactions on Information Theory, vol. 28, no. 4, pp. 565- 577, 1982. (Cited on pages 15 and 27.)

HaRUYAMA, S.; ZHAO, Q. Designing smaller decision trees using multiple objective optimization based gps. In: IEEE International Conference on Systems, Man and Cybernetics, p. 5 pp., 2002. (Cited on pages 67 and 83.)

Haskell, R.; Noui-Mehidi, A. Design of hierarchical classifiers. In: Sherwani, N.; De DoncKer, E.; KAPEnga, J., eds. Computing in the 90's, vol. 507 of Lecture Notes in Computer Science, Springer Berlin / Heidelberg, pp. 118-124, 1991. (Cited on pages 19 and 27.)

Hastie, T.; Tibshirani, R.; Friedman, J. The elements of statistical learning: Data mining, inference, and prediction, second edition. Springer Series in Statistics, 2nd ed. 2009. corr. 3rd printing edn.. Springer, 2009. (Cited on page 55.)

Hauska, H.; Swain, P. The decision tree classifier: design and potential. 2nd Symposium on Machine Processing of Remotely Sensed Data, 1975. (Cited on page 10.)

HAZAN, A.; RAmiRez, R. Modelling expressive performance using consistent evolutionary regression trees. In: 17th European Conference on Artificial Intelligence (Workshop on Evolutionary Computation), Riva del Garda, Italy, 2006. (Cited on page 75.)

Hazan, A.; Ramirez, R.; Maestre, E.; Perez, A.; Pertusa, A. Modelling Expressive Performance: A Regression Tree Approach Based on Strongly Typed Genetic Programming. In: Applications of Evolutionary Computing, vol. 3907 of Lecture Notes in Computer Science, Springer Berlin / Heidelberg, pp. 676-687, 2006. (Cited on pages xii and 75.)

Heath, D.; Kasif, S.; Salzberg, S. Induction of oblique decision trees. Journal of Artificial Intelligence Research, vol. 2, pp. 1-32, 1993. (Cited on pages 26, 28, and 32.) 


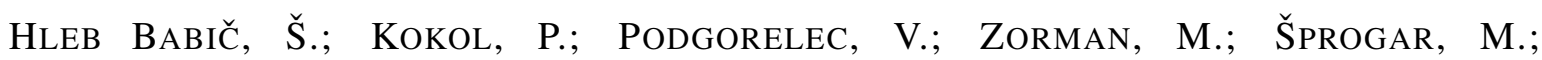
Molan ŠTiglic, M. The art of building decision trees. Journal of Medical Systems, vol. 24, pp. 43-52, 2000. (Cited on pages 64, 66, 67, and 68.)

Ho, T.; BASU, M. Complexity measures of supervised classification problems. IEEE Transactions on Pattern Analysis and Machine Intelligence, vol. 24, no. 3, pp. 289-300, 2002. (Cited on pages 87 and 101.)

Ho, T.; BASU, M.; LAw, M. Measures of geometrical complexity in classification problems. In: Data Complexity in Pattern Recognition, Springer London, 2006. (Cited on pages 87 and 101.)

Holland, J. H. Adaptation in natural and artificial systems. Cambridge, MA, USA: MIT Press, 1975. (Cited on page 46.)

HsiaO, W.; SHIH, Y. Splitting variable selection for multivariate regression trees. Statistics and Probability Letters, vol. 77, no. 3, pp. 265-271, 2007. (Cited on page 32.)

Hunt, E. B.; MARin, J.; Stone, P. J. Experiments in induction. New York, NY, USA: Academic Press, 1966. (Cited on pages 10 and 12.)

HyAFIL, L.; RIVEST, R. Constructing optimal binary decision trees is np-complete. Information Processing Letters, vol. 5, no. 1, pp. 15-17, 1976. (Cited on page 11.)

IMAN, R.; DAVENPORT, J. Approximations of the critical region of the friedman statistic. Communications in Statistics, pp. 571-595, 1980. (Cited on page 107.)

ItTner, A. Non-linear decision trees. In: 13th International Conference on Machine Learning, pp. 1-6, 1996. (Cited on pages xi, 30, and 31.)

Johansson, U.; Niklasson, L. Evolving decision trees using oracle guides. In: IEEE Symposium on Computational Intelligence and Data Mining (CIDM 2009), pp. 238-244, 2009. (Cited on pages 61, 64, 66, 69, and 70.)

JONG, K. D.; SpEARS, W. An analysis of the interacting roles of population size and crossover in genetic algorithms. In: 1st Workshop on Parallel Problem Solving from Nature, London, UK, pp. 38-47, 1991. (Cited on page 69.)

Jun, B.; KIM, C.; Song, Y.-Y.; KIM, J. A New Criterion in Selection and Discretization of Attributes for the Generation of Decision Trees. IEEE Transactions on Pattern Analysis and Machine Intelligence, vol. 19, no. 2, pp. 1371-1375, 1997. (Cited on pages 16, 17, 27, and 92.)

KALKANIS, G. The application of confidence interval error analysis to the design of decision tree classifiers. Pattern Recognition Letters, vol. 14, no. 5, pp. 355-361, 1993. (Cited on pages 24 and 27.) 
Kalles, D.; PAPAgElis, A. Lossless fitness inheritance in genetic algorithms for decision trees. Soft Computing - A Fusion of Foundations, Methodologies and Applications, vol. 14, pp. 973-993, 2010. (Cited on page 86.)

KARALIČ, A. Employing linear regression in regression tree leaves. In: 10th European conference on Artificial intelligence, ECAI '92, New York, NY, USA: John Wiley \& Sons, Inc., pp. 440-441, 1992 (ECAI'92, ). (Cited on page 39.)

KASS, G. V. An exploratory technique for investigating large quantities of categorical data. APPL STATIST, vol. 29, no. 2, pp. 119-127, 1980. (Cited on page 10.)

KDNugGeTs Poll: Data mining/analytic methods you used frequently in the past 12 months. 2007.

Available at http://www.kdnuggets.com/polls/2007/data_mining_ methods.htm (Cited on page 1.)

KeAne, A.; Brown, S. The design of a satellite boom with enhanced vibration performance using genetic algorithm techniques. In: Conference on Adaptative Computing in Engineering Design and Control, Plymouth, UK, pp. 107-113, 1996. (Cited on pages 3 and 5.)

Kennedy, H.; Chinniah, C.; Bradbeer, P.; Morss, L. The contruction and evaluation of decision trees: A comparison of evolutionary and concept learning methods. In: CORNE, D.; ShapIRo, J., eds. Evolutionary Computing, vol. 1305 of Lecture Notes in Computer Science, Springer Berlin / Heidelberg, pp. 147-161, 1997. (Cited on pages xi, 59, 61, 63, 66, 67, 68, and 87.)

KhoshgoftaAr, T.; LiU, Y. A multi-objective software quality classification model using genetic programming. IEEE Transactions on Reliability, vol. 56, no. 2, pp. 237 -245, 2007. (Cited on pages 83 and 85.)

KhoshgoftaAr, T. M.; LiU, Y.; Seliya, N. Genetic programming-based decision trees for software quality classification. Tools with Artificial Intelligence, IEEE International Conference on, vol. 0, p. 374, 2003. (Cited on pages 83 and 84.)

Kim, B.; LAndgrebe, D. Hierarchical classifier design in high-dimensional numerous class cases. IEEE Transactions on Geoscience and Remote Sensing, vol. 29, no. 4, pp. 518-528, 1991. (Cited on pages 2 and 42.)

KIM, D. Structural risk minimization on decision trees using an evolutionary multiobjective optimization. In: KeiJzer, M.; O’Reilly, U.-M.; LuCAS, S. M.; Costa, E.; Soule, T., eds. Genetic Programming, vol. 3003 of Lecture Notes in Computer Science, Springer Berlin / Heidelberg, pp. 338-348, 2004. (Cited on pages 61, 65, 66, 67, 68, 69, 70, and 87.) 
Kim, K. M.; Park, J. J.; Song, M. H.; Kim, C.; Suen, C. Y. Binary decision tree using genetic algorithm for recognizing defect patterns of cold mill strip. In: TAWFIK, A. Y.; Goodwin, S. D., eds. Advances in Artificial Intelligence, vol. 3060 of Lecture Notes in Computer Science, Springer Berlin, pp. 461-466, 2004a. (Cited on pages 56, 78, and 83.)

Kim, K. M.; PARK, J. J.; Song, M. H.; Kim, C.; Suen, C. Y. Binary decision tree using k-means and genetic algorithm for recognizing defect patterns of cold mill strip. In: IEA/AIE'2004: 17th international conference on Innovations in applied artificial intelligence, Springer Verlag Inc, pp. 341-350, 2004b. (Cited on pages 56, 78, and 83.)

Kokol, P.; Podgorelec, V.; Pighin, M. Using software metrics and evolutionary decision trees for software quality control. In: European Software Control and Metrics (ESCOM '01), pp. 181-191, 2001. (Cited on page 84.)

Kononenko, I. Estimating attributes: analysis and extensions of RELIEF. In: Proceedings of the European conference on machine learning on Machine Learning, Secaucus, NJ, USA: Springer-Verlag New York, Inc., pp. 171-182, 1994. (Cited on pages 24 and 27.)

Kononenko, I.; Bratko, I.; Roskar, E. Experiments in automatic learning of medical diagnostic rules. Tech. Rep., Jozef Stefan Institute, Ljubljana, Yugoslavia, 1984. (Cited on pages 10,40 , and 94.)

KozA, J. A genetic approach to the truck backer upper problem and the inter-twined spiral problem. In: International Joint Conference on Neural Networks (IJCNN 92), pp. 310 -318, 1992a. (Cited on page 71.)

KozA, J. R. Concept formation and decision tree induction using the genetic programming paradigm. In: PPSN I: 1st Workshop on Parallel Problem Solving from Nature, London, UK: Springer-Verlag, pp. 124-128, 1991. (Cited on page 62.)

KoZA, J. R. Genetic programming: on the programming of computers by means of natural selection. Cambridge, MA, USA: MIT Press, 1992b. (Cited on pages 3, 46, 49, and 71.)

Kramer, O. Self-adaptive crossover. In: Self-Adaptive Heuristics for Evolutionary Computation, vol. 147 of Studies in Computational Intelligence, Springer Berlin Heidelberg, pp. 97-113, 2008. (Cited on page 169.)

Kretowski, M. An evolutionary algorithm for oblique decision tree induction. In: Rutkowski, L.; Siekmann, J.; Tadeusiewicz, R.; Zadeh, L. A., eds. Artificial Intelligence and Soft Computing - ICAISC 2004, vol. 3070 of Lecture Notes in Computer Science, Springer Berlin / Heidelberg, pp. 432-437, 2004. (Cited on page 79.)

Kretowski, M.; Czajkowski, M. An evolutionary algorithm for global induction of regression trees. In: RUtKowsKi, L.; SCHERER, R.; TADEUSIEWICZ, R.; ZADEH, L.; 
Zurada, J., eds. Artifical Intelligence and Soft Computing, vol. 6114 of Lecture Notes in Computer Science, Springer Berlin / Heidelberg, pp. 157-164, 2010. (Cited on page 74.)

Kretowski, M.; Grzes, M. Global induction of oblique decision trees: An evolutionary approach. In: Klopotek, M.; WierzChon, S.; Trojanowski, K., eds. Intelligent Information Processing and Web Mining, vol. 31 of Advances in Soft Computing, Springer Berlin / Heidelberg, pp. 309-318, 2005. (Cited on pages xi and 72.)

Kretowski, M.; Grzes, M. Evolutionary induction of cost-sensitive decision trees. In: Esposito, F.; Ras, Z.; Malerba, D.; Semeraro, G., eds. Foundations of Intelligent Systems, vol. 4203 of Lecture Notes in Computer Science, Springer Berlin / Heidelberg, pp. 121-126, 2006a. (Cited on pages 61, 64, 67, and 68.)

Kretowski, M.; Grzes, M. Mixed decision trees: An evolutionary approach. In: 8th International Conference on Data Warehousing and Knowledge Discovery (DaWaK 2006), Krakow, Poland, pp. 260-269, 2006b. (Cited on pages 61, 64, 67, 68, 69, and 87.)

Kretowski, M.; Grzes, M. Evolutionary induction of decision trees for misclassification cost minimization. In: 8th International Conference on Adaptive and Natural Computing Algorithms, Part I, Berlin, Heidelberg: Springer-Verlag, pp. 1-10, 2007a. (Cited on pages 61, $64,66,67,68$, and 69.)

Kretowski, M.; GrZES, M. Evolutionary induction of decision trees for misclassification cost minimization. In: Beliczynski, B.; Dzielinski, A.; IwANowski, M.; Ribeiro, B., eds. Adaptive and Natural Computing Algorithms, vol. 4431 of Lecture Notes in Computer Science, Springer Berlin / Heidelberg, pp. 1-10, 2007b. (Cited on pages 61, 64, 66, 67, 68, and 69.)

KuO, C.-S.; Hong, T.-P.; CHen, C.-L. Applying genetic programming technique in classification trees. Soft Computing, vol. 11, no. 12, pp. 1165-1172, 2007. (Cited on page 83.)

Kuo, C.-S.; Hong, T.-P.; CHEN, C.-L. An improved knowledge-acquisition strategy based on genetic programming. Cybernetics and Systems, vol. 39, no. 7, pp. 672-685, 2008. (Cited on page 83.)

Landeweerd, G.; Timmers, T.; Gelsema, E.; Bins, M.; Halie, M. Binary tree versus single level tree classification of white blood cells. Pattern Recognition, vol. 16, no. 6, pp. 571-577, 1983. (Cited on pages 1, 41, and 167.)

Larsen, D. R.; Speckman, P. L. Multivariate Regression Trees for Analysis of Abundance Data. Biometrics, vol. 60, no. 2, pp. 543-549, 2004. (Cited on page 32.)

LeE, Y.-S. A New Splitting Approach for Regression Trees. Tech. Rep., Dongguk University, Department of Statistics, Dongguk University, Department of Statistics, 2001. (Cited on pages 26 and 27.) 
LI, J.; LI, X.; YAO, X. Cost-sensitive classification with genetic programming. In: 2005 IEEE Congress on Evolutionary Computation, pp. 2114 - 2121, 2005. (Cited on pages 64, 66, 69, and 70.)

Li, X.; Dubes, R. C. Tree classifier design with a permutation statistic. Pattern Recognition, vol. 19, no. 3, pp. 229-235, 1986. (Cited on pages 21 and 27.)

Li, X.-B.; Sweigart, J. R.; Teng, J. T. C.; Donohue, J. M.; Thombs, L. A.; Wang, S. M. Multivariate decision trees using linear discriminants and tabu search. IEEE Transactions on Systems, Man, and Cybernetics - Part A: Systems and Humans, vol. 33, no. 2, pp. 194-205, 2003. (Cited on page 32.)

Liu, H.; Setiono, R. Feature transformation and multivariate decision tree induction. In: Discovey Science, pp. 279-291, 1998. (Cited on pages 30 and 32.)

Llora, X.; Garrell, J. M. Evolution of decision trees. In: 4th Catalan Conference on Artificial Intelligence, ACIA Press, pp. 115-122, 2001. (Cited on pages 61, 62, 66, 67, and 68.)

LlorÀ, X.; Wilson, S. W. Mixed Decision Trees: Minimizing Knowledge Representation Bias in LCS. In: Genetic and Evolutionary Computation (GECCO 2004), vol. 3103 of Lecture Notes in Computer Science, Springer Berlin / Heidelberg, pp. 797-809, 2004. (Cited on pages 62, 66, 67, 68, and 87.)

LLOYD, S. Least squares quantization in pcm. IEEE Transactions on Information Theory, vol. 28, no. 2, pp. 129 - 137, 1982. (Cited on page 76.)

Lobo, J. M.; Jiménez-VAlverde, A.; ReAl, R. AUC: a misleading measure of the performance of predictive distribution models. Global Ecology and Biogeography, vol. 17, no. 2, pp. 145-151, 2008. (Cited on page 146.)

LOH, W. Regression trees with unbiased variable selection and interaction detection. Statistica Sinica, vol. 12, pp. 361-386, 2002. (Cited on pages 26 and 27.)

LOH, W.; SHIH, Y. Split selection methods for classification trees. Statistica Sinica, vol. 7, pp. 815-840, 1997. (Cited on pages 24, 27, 40, and 94.)

LUKE, S. Two fast tree-creation algorithms for genetic programming. IEEE Transactions on Evolutionary Computation, vol. 4, no. 3, pp. 274-283, 2000. (Cited on page 49.)

MA, C. Y.; WANG, X. Z. Inductive data mining based on genetic programming:automatic generation of decision trees from data for process historical data analysis. Computers and Chemical Engineering, vol. 33, no. 10, pp. 1602 - 1616, 2009. (Cited on pages 61, 62, 63, 66, 67, 68, and 70.) 
Majeed, H.; Ryan, C. A Less Destructive, Context-Aware Crossover Operator for GP. In: Collet, P.; Tomassini, M.; Ebner, M.; Gustafson, S.; EkÁRt, A., eds. Lecture Notes in Computer Science, Springer Berlin / Heidelberg SN, pp. 36-48, 2006a. (Cited on page 52.)

MAJEED, H.; RYAN, C. Using context-aware crossover to improve the performance of GP. In: 8th Annual Conference on Genetic and Evolutionary Computation (GECCO '06), ACM, pp. 847-854, 2006b. (Cited on page 52.)

Malerba, D.; Esposito, F.; Ceci, M.; Appice, A. Top-down induction of model trees with regression and splitting nodes. IEEE Transactions on Pattern Analysis and Machine Intelligence, pp. 612-625, 2004. (Cited on pages 26 and 27.)

Mangasarian, O.; Setiono, R.; Wolberg, W. H. Pattern Recognition Via Linear Programming: Theory And Application To Medical Diagnosis. In: SIAM Workshop on Optimization, 1990. (Cited on page 32.)

Manwani, N.; Sastry, P. A Geometric Algorithm for Learning Oblique Decision Trees. In: Chaudhury, S.; Mitra, S.; Murthy, C.; Sastry, P.; Pal, S., eds. Pattern Recognition and Machine Intelligence, Berlin, Heidelberg: Springer Berlin / Heidelberg, pp. 25-31, 2009. (Cited on page 32.)

Marín-Blázquez, J. G.; Schulenburg, S. A hyper-heuristic framework with xcs: learning to create novel problem-solving algorithms constructed from simpler algorithmic ingredients. In: Proceedings of the 2003-2005 international conference on Learning classifier systems, IWLCS'03-05, Berlin, Heidelberg: Springer-Verlag, pp. 193-218, 2007 (IWLCS'03-05, ). (Cited on page 53.)

Martin, J. An exact probability metric for decision tree splitting and stopping. Machine Learning, vol. 28, no. 2, pp. 257-291, 1997. (Cited on pages 23, 27, and 92.)

Mason, S. J.; Graham, N. E. Areas beneath the relative operating characteristics (roc) and relative operating levels (rol) curves: Statistical significance and interpretation. Quarterly Journal of the Royal Meteorological Society, vol. 128, no. 584, pp. 2145-2166, 2002. (Cited on page 146.)

Mckay, R.; HoAi, N.; Whigham, P.; Shan, Y.; O NeILl, M. Grammar-based Genetic Programming: a survey. Genetic Programming and Evolvable Machines, vol. 11, no. 3, pp. 365-396, 2010. (Cited on page 170.)

Michalewicz, Z.; Schmidt, M. Parameter control in practice. In: Lobo, F.; Lima, C.; Michalewicz, Z., eds. Parameter Setting in Evolutionary Algorithms, vol. 54 of Studies in Computational Intelligence, Springer Berlin / Heidelberg, pp. 277-294, 2007. (Cited on page 68.) 
Mingers, J. Expert systems - rule induction with statistical data. Journal of the Operational Research Society, vol. 38, pp. 39-47, 1987. (Cited on pages 16, 27, 36, and 92.)

Mingers, J. An empirical comparison of pruning methods for decision tree induction. Machine Learning, vol. 4, no. 2, pp. 227-243, 1989a. (Cited on page 38.)

Mingers, J. An empirical comparison of selection measures for decision-tree induction. Machine Learning, vol. 3, no. 4, pp. 319-342, 1989b. (Cited on pages 16, 19, 27, and 92.)

Mitchell, M. An introduction to genetic algorithms. Cambridge, MA, USA: MIT Press, 1998. (Cited on page 48.)

Mitchell, T. M. Machine learning. New York: McGraw-Hill, 1997. (Cited on page 11.)

Mola, F.; Siciliano, R. A fast splitting procedure for classification trees. Statistics and Computing, vol. 7, no. 3, pp. 209-216, 1997. (Cited on pages 21 and 27.)

Montana, D. J. Strongly typed genetic programming. Evolutionary Computation, vol. 3, no. 2, pp. 199-230, 1995. (Cited on page 71.)

Monti, S.; TAmayo, P.; Mesirov, J.; Golub, T. Consensus clustering: A resampling-based method for class discovery and visualization of gene expression microarray data. Machine Learning, vol. 52, no. 1-2, pp. 91-118, 2003. (Cited on page 112.)

Morgan, J. N.; Messenger, R. C. THAID: a sequential search program for the analysis of nominal scale dependent variables. Tech. Rep., Institute for Social Research, University of Michigan, 1973. (Cited on page 9.)

MurThy, S. K. Automatic construction of decision trees from data: A multi-disciplinary survey. Data Mining and Knowledge Discovery, vol. 2, no. 4, pp. 345-389, 1998. (Cited on pages 9, 10, and 56.)

Murthy, S. K.; Kasif, S.; Salzberg, S.; Beigel, R. OC1: A Randomized Induction of Oblique Decision Trees. In: Proceedings of the 11th National Conference on Artificial Intelligence (AAAI '93), pp. 322-327, 1993. (Cited on pages 28, 32, 43, and 79.)

Murthy, S. K.; Kasif, S.; Salzberg, S. S. A System for Induction of Oblique Decision Trees. Journal of Artificial Intelligence Research, vol. 2, pp. 1-32, 1994. (Cited on pages 26, 28, 32, and 43.)

Murthy, S. K.; SAlzberg, S. Lookahead and pathology in decision tree induction. In: 14th International Joint Conference on Artificial Intelligence, Morgan Kaufmann, pp. 1025-1031, 1995. (Cited on page 42.) 
Naumov, G. E. Np-completeness of problems of construction of optimal decision trees. Soviet Physics: Doklady, vol. 36, no. 4, 1991. (Cited on page 11.)

Niblett, T.; BratKo, I. Learning decision rules in noisy domains. In: 6th Annual Technical Conference on Research and Development in Expert Systems III, pp. 25-34, 1986. (Cited on pages $35,36,39,95$, and 96.)

NiIMI, A.; TAZAKI, E. Genetic programming combined with association rule algorithm for decision tree construction. In: 4th International Conference on Knowledge-Based Intelligent Engineering Systems and Allied Technologies, pp. 746 -749, 2000. (Cited on pages 66, 67, and 69.)

Nikolaev, N.; Slavov, V. Inductive genetic programming with decision trees. In: VAN SOMEREN, M.; WIDMER, G., eds. Machine Learning (ECML-97), vol. 1224 of Lecture Notes in Computer Science, Springer Berlin / Heidelberg, pp. 183-190, 1997. (Cited on pages 64, 66, 67, and 69.)

NiLsSOn, N. J. The mathematical foundations of learning machines. San Francisco, CA, USA: Morgan Kaufmann Publishers Inc., 1990. (Cited on page 28.)

Norton, S. W. Generating better decision trees. In: 11th International Joint Conference on Artificial Intelligence, Detroit, Michigan: Morgan Kaufmann Publishers Inc., pp. 800-805, 1989. (Cited on page 42.)

Nunez, M. The use of background knowledge in decision tree induction. Machine Learning, vol. 6, no. 3, pp. 231-250, 1991. (Cited on page 82.)

OchoA, G.; QU, R.; BURKe, E. K. Analyzing the landscape of a graph based hyper-heuristic for timetabling problems. In: Proceedings of the 11th Annual conference on Genetic and evolutionary computation, GECCO '09, New York, NY, USA: ACM, pp. 341-348, 2009a (GECCO '09, ). (Cited on page 53.)

Ochoa, G.; Vazquez-Rodriguez, J.; Petrovic, S.; Burke, E. Dispatching rules for production scheduling: A hyper-heuristic landscape analysis. In: IEEE Congress on Evolutionary Computation, pp. 1873-1880, 2009b. (Cited on page 53.)

Oganov, A. R.; Chen, J.; Gatti, C.; Ma, Y.; Ma, Y.; Glass, C. W.; LiU, Z.; Yu, T.; Kurakevych, O. O.; Solozhenko, V. L. Ionic high-pressure form of elemental boron. Nature, vol. 457, pp. 863-867, 2009. (Cited on pages 3 and 5.)

OKA, S.; ZHAO, Q. Design of decision trees through integration of C4.5 and GP. In: 4th Jpn.-Australia Joint Workshop Intell. Evol. Syst., pp. 128-135, 2000. (Cited on pages 66, 67, 68, and 83.) 
OlteAn, M. Evolving evolutionary algorithms using linear genetic programming. Evolutionary Computation, vol. 13, no. 3, pp. 387-410, 2005. (Cited on page 54.)

OseI-Bryson, K. Post-pruning in regression tree induction: An integrated approach. Expert Systems with Applications, vol. 34, no. 2, pp. 1481-1490, 2008. (Cited on page 39.)

ÖZCAn, E.; Bilgin, B.; Korkmaz, E. E. A comprehensive analysis of hyper-heuristics. Intelligent Data Analysis, vol. 12, no. 1, pp. 3-23, 2008. (Cited on page 52.)

PAGE, D.; RAY, S. Skewing: an efficient alternative to lookahead for decision tree induction. In: 18th International Joint Conference on Artificial Intelligence, Morgan Kaufmann Publishers Inc., pp. 601-607, 2003. (Cited on page 43.)

Palaniappan, K.; Zhu, F.; ZhUang, X.; Zhao, Y.; Blanchard, A. Enhanced binary tree genetic algorithm for automatic land cover classification. In: IEEE International Geoscience and Remote Sensing Symposium (IGARSS 2000), pp. 688 -692, 2000. (Cited on page 78.)

Papagelis, A.; Kalles, D. Ga tree: genetically evolved decision trees. Tools with Artificial Intelligence, IEEE International Conference on, vol. 0, p. 0203, 2000. (Cited on pages 61, 64, 67, 68, and 69.)

Papagelis, A.; Kalles, D. Breeding decision trees using evolutionary techniques. In: 18th International Conference on Machine Learning, San Francisco, CA, USA: Morgan Kaufmann Publishers Inc., pp. 393-400, 2001. (Cited on pages 58, 61, 64, 67, 68, 69, and 87.)

PAPPA, G.; FreitAs, A. Automatically evolving rule induction algorithms. In: 17th European Conference on Machine Learning, pp. 341-352, 2006. (Cited on pages 4, 5, and 89.)

PAPPA, G. L. Automatically Evolving Rule Induction Algorithms with Grammar-Based Genetic Programming. Ph.D. thesis, University of Kent at Canterbury, 2007. (Cited on pages 89, 100, 104, and 146.)

Pappa, G. L.; Freitas, A. A. Automating the Design of Data Mining Algorithms: An Evolutionary Computation Approach. Springer Publishing Company, Incorporated, 2009a. (Cited on pages 3, 4, 5, 54, and 104.)

PAPPA, G. L.; Freitas, A. A. Evolving rule induction algorithms with multi-objective grammar-based genetic programming. Knowledge and Information Systems, vol. 19, pp. 283-309, 2009b. (Cited on page 104.)

Pappa, G. L.; Ochoa, G.; Hyde, M. R.; Freitas, A. A.; Woodward, J.; Swan, J. Contrasting meta-learning and hyper-heuristic research: the role of evolutionary algorithms. Genetic Programming and Evolvable Machines, 2013. (Cited on page 3.) 
Patterson, A.; Niblett, T. ACLS user manual. Glasgow: Intelligent Terminals Ltd, 1983. (Cited on page 10.)

Pattipati, K.; Alexandridis, M. Application of heuristic search and information theory to sequential fault diagnosis. IEEE Transactions on Systems, Man and Cybernetics, vol. 20, pp. 872-887, 1990. (Cited on page 18.)

PILLAY, N. An analysis of representations for hyper-heuristics for the uncapacitated examination timetabling problem in a genetic programming system. In: Proceedings of the 2008 annual research conference of the South African Institute of Computer Scientists and Information Technologists on IT research in developing countries: riding the wave of technology, SAICSIT '08, New York, NY, USA: ACM, pp. 188-192, 2008 (SAICSIT '08, ). (Cited on page 53.)

Podgorelec, V.; Hericko, M.; Rozman, I. Improving mining of medical data by outliers prediction. In: 18th IEEE Symposium on Computer-Based Medical Systems, pp. 91 - 96, 2005. (Cited on pages 83 and 84.)

Podgorelec, V.; KoKol, P. Evolutionary construction of medical decision trees. In: 20th Annual International Conference of the IEEE Engineering in Medicine and Biology Society, pp. $1202-1205$ vol.3, 1998. (Cited on pages 83 and 84.)

Podgorelec, V.; KoKol, P. Self-adapting evolutionary decision support model. In: IEEE International Symposium on ndustrial Electronics (ISIE '99), pp. $1484-1489$, 1999. (Cited on pages $64,66,67$, and 68.)

Podgorelec, V.; KoKOL, P. Evolutionary decision forests-decision making with multiple evolutionary constructed decision trees. In: PRESS, W., ed. Problems in Applied Mathematics and Computational Intelligence, pp. 97-103, 2001a. (Cited on pages 64, 66, 67, 68, and 83.)

Podgorelec, V.; KoKOL, P. Towards more optimal medical diagnosing with evolutionary algorithms. Journal of Medical Systems, vol. 25, pp. 195-219, 2001b. (Cited on pages 64, 66, $67,68,69,70,83$, and 84 .)

Podgorelec, V.; KoKol, P. Evolutionary induced decision trees for dangerous software modules prediction. Information Processing Letters, vol. 82, no. 1, pp. 31 - 38, 2002. (Cited on pages $56,59,83$, and 84 .)

Potgieter, G.; Engelbrecht, A. Genetic algorithms for the structural optimisation of learned polynomial expressions. Applied Mathematics and Computation, vol. 186, no. 2, pp. 1441-1466, 2007. (Cited on page 76.)

Potgieter, G.; Engelbrecht, A. Evolving model trees for mining data sets with continuous-valued classes. Expert Systems with Applications, vol. 35, pp. 1513-1532, 2008. (Cited on page 76.) 
POWERS, D. Evaluation: From precision, recall and f-measure to ROC, informedness, markedness \& correlation. Journal of Machine Learning Technologies, vol. 2, no. 1, pp. 37-63, 2011. (Cited on page 145.)

QUiNLAN, J. R. Discovering rules by induction from large collections of examples. In: MichIE, D., ed. Expert Systems in the Micro-elect Age, Edinburgh: Edinburgh University Press, 1979. (Cited on pages 10 and 13.)

QUINLAN, J. R. Induction of decision trees. Machine Learning, vol. 1, no. 1, pp. 81-106, 1986. (Cited on pages 13, 15, 16, 27, 33, 40, 55, 81, 92, 94, and 95.)

Quinlan, J. R. Decision trees as probabilistic classifiers. In: 4th International Workshop on Machine Learning, 1987a. (Cited on pages 41 and 95.)

Quinlan, J. R. Simplifying decision trees. International Journal of Man-Machine Studies, vol. 27, pp. 221-234, 1987b. (Cited on pages 33, 34, 38, 95, and 96.)

QUINLAN, J. R. Unknown attribute values in induction. In: 6th International Workshop on Machine Learning, pp. 164-168, 1989. (Cited on pages 40, 41, 94, and 95.)

Quinlan, J. R. Learning with Continuous Classes. In: 5th Australian Joint Conference on Artificial Intelligence, pp. 343-348, 1992. (Cited on pages 25, 27, and 74.)

QUINLAN, J. R. C4.5: programs for machine learning. San Francisco, CA, USA: Morgan Kaufmann, 1993. (Cited on pages 1, 5, 13, 16, 27, 33, 37, 44, 55, 80, 81, 92, 94, 95, 96, 106, and 165.)

Quinlan, J. R. MDL and Categorcal Theories (continued). In: Twelfth International Conference in Machine Learning, Morgan Kaufmann, pp. 464-470, 1995. (Cited on page 64.)

Quinlan, J. R.; RIVEST, R. L. Inferring decision trees using the minimum description length principle. Information and Computation, vol. 80, no. 3, pp. 227-248, 1989. (Cited on pages 22, 23, and 27.)

REYNOLDS, R.; AL-SHEHRI, H. The use of cultural algorithms with evolutionary programming to guide decision tree induction in large databases. In: IEEE International Conference on Evolutionary Computation, pp. 541 -546, 1998. (Cited on pages 61, 64, and 66.)

Robnik-Sikonja, M.; Kononenko, I. Pruning Regression Trees with MDL. In: European conference on artificial intelligence, pp. 455-459, 1998. (Cited on page 39.)

Rokach, L.; Maimon, O. Top-down induction of decision trees classifiers - a survey. IEEE Transactions on Systems, Man, and Cybernetics, Part C: Applications and Reviews, vol. 35, no. 4, pp. $476-487,2005$. (Cited on pages xv, 1, 13, 26, 32, and 56.) 
Rounds, E. M. A combined nonparametric approach to feature selection and binary decision tree design. Pattern Recognition, vol. 12, no. 5, pp. 313-317, 1980. (Cited on pages 19 and 27.)

Rouwhorst, S.; Engelbrecht, A. Searching the forest: using decision trees as building blocks for evolutionary search in classification databases. In: IEEE Congress on Evolutionary Computation (CEC 2000), pp. 633 -638, 2000. (Cited on pages 61, 64, 66, 67, 68, 69, and 70.)

SÁ, J. P.; Gama, J.; Sebastião, R.; Alexandre, L. A. Decision Trees Using the Minimum Entropy-of-Error Principle. In: 13th International Conference on Computer Analysis of Images and Patterns, Springer-Verlag, pp. 799-807, 2009. (Cited on pages 17 and 27.)

Safavian, S.; Landgrebe, D. A survey of decision tree classifier methodology. IEEE Transactions on Systems, Man and Cybernetics, vol. 21, no. 3, pp. 660 -674, 1991. (Cited on pages 10 and 56.)

SchWARZ, G. Estimating the dimension of a model. Annals of Statistics, vol. 6, pp. 461-464, 1978. (Cited on page 73.)

SENI, G.; ELDER, J. Ensemble methods in data mining: Improving accuracy through combining predictions. Morgan and Claypool Publishers, 2010. (Cited on page 55.)

Sethi, I. K.; Sarvarayudu, G. P. R. Hierarchical Classifier Design Using Mutual Information. IEEE Transactions on Pattern Analysis and Machine Intelligence, vol. 4, no. 4, pp. 441-445, 1982. (Cited on pages 14 and 27.)

Shah, S.; SAStry, P. New algorithms for learning and pruning oblique decision trees. IEEE Transactions on Systems, Man, and Cybernetics, Part C: Applications and Reviews, vol. 29, no. 4, pp. $494-505$, 1999. (Cited on pages 31, 32, 56, and 81.)

Shali, A.; Kangavari, M.; Bina, B. Using genetic programming for the induction of oblique decision trees. In: 6th International Conference on Machine Learning and Applications (ICMLA 2007), pp. $38-43,2007$. (Cited on page 80.)

SHANnOn, C. E. A mathematical theory of communication. BELL System Technical Journal, vol. 27, no. 1, pp. 379-423, 625-56, 1948. (Cited on pages 14 and 92.)

SHIH, Y. Selecting the best categorical split for classification trees. Statistics and Probability Letters, vol. 54, pp. 341-345, 2001. (Cited on page 18.)

Shirasaka, M.; Zhao, Q.; Hammami, O.; Kuroda, K.; Saito, K. Automatic design of binary decision trees based on genetic programming. In: Second Asia-Pacific Conference on Simulated Evolution and Learning, 1998. (Cited on pages 58, 61, 65, 66, 67, 68, 69, 70, and 83.) 
SIEGEL, E. V. Competitively evolving decision trees against fixed training cases for natural language processing Cambridge, MA, USA: MIT Press, pp. 409-423, 1994. (Cited on pages 83 and 84.)

Silva, L. M.; Felgueiras, C. S.; Alexandre, L. A.; Marques de Sá, J. Error entropy in classification problems: a univariate data analysis. Neural computation, vol. 18, no. 9, pp. 2036-2061, 2006. (Cited on page 17.)

Slavov, V.; Nokolaev, N. I. Fitness landscapes and inductive genetic programming. In: 3rd International Conference on Artificial Neural Nets and Genetic Algorithms, Berlin, Germany: Springer-Verlag, pp. 414-418, 1997. (Cited on page 87.)

Smith, S. F. Rna search acceleration with genetic algorithm generated decision trees. In: ICMLA '08: 2008 Seventh International Conference on Machine Learning and Applications, Washington, DC, USA: IEEE Computer Society, pp. 565-570, 2008. (Cited on pages 60, 67, 83, and 84.)

Smith-Miles, K. A. Cross-disciplinary perspectives on meta-learning for algorithm selection. ACM Computing Surveys, vol. 41, pp. 6:1-6:25, 2009. (Cited on page 3.)

Sonquist, J. A.; Baker, E. L.; Morgan, J. N. Searching for structure. Tech. Rep., Institute for Social Research, University of Michigan, 1971. (Cited on page 9.)

Sorensen, K.; Janssens, G. K. Data mining with genetic algorithms on binary trees. European Journal of Operational Research, vol. 151, no. 2, pp. 253-264, 2003. (Cited on pages $59,61,67,68$, and 83 .)

Souto, M.; Costa, I.; De Araujo, D.; Ludermir, T.; SchlieP, A. Clustering cancer gene expression data: a comparative study. BMC Bioinformatics, vol. 9, no. 1, p. 497, 2008. (Cited on pages $6,111,112$, and 166 .)

Sprogar, M.; Kokol, P.; Zorman, M.; Podgorelec, V.; Lhotska, L.; Klema, J. Evolving groups of basic decision trees. In: CBMS '01: Fourteenth IEEE Symposium on Computer-Based Medical Systems, Washington, DC, USA: IEEE Computer Society, p. 183, 2001. (Cited on pages 64 and 67.)

StANLEy, K. O.; MiIKKUlainen, R. Evolving neural networks through augmenting topologies. Evolutionary Computation, vol. 10, no. 2, pp. 99-127, 2002. (Cited on pages 4 and 54.)

StORER, R. H.; WU, S. D.; VACCARI, R. New search spaces for sequencing problems with application to job shop scheduling. Management Science, vol. 38, no. 10, pp. 1495-1509, 1992. (Cited on page 53.)

TAlmon, J. A multiclass nonparametric partitioning algorithm. Pattern Recognition Letters, vol. 4, no. 1, pp. 31-38, 1986. (Cited on pages 14 and 27.) 
TAN, P. J.; Dowe, D. L. MML inference of oblique decision trees. In: 17th Australian Joint Conference on AI, pp. 1082-1088, 2004. (Cited on page 32.)

TAn, P.-N.; Steinbach, M.; Kumar, V. Introduction to data mining. Addison-Wesley, 2005. (Cited on pages xii, 12, and 145.)

TANigaWA, T.; ZhaO, Q. A study on efficient generation of decision trees using genetic programming. In: Genetic and Evolutionary Computation Conference (GECCO 2000), Morgan Kaufmann, pp. 1047-1052, 2000. (Cited on pages 58, 61, 65, 66, 67, 68, 69, 70, and 83.)

TAYLOR, P.; JONES, M. Splitting criteria for regression trees. Journal of Statistical Computation and Simulation, vol. 55, no. 4, pp. 267-285, 1996. (Cited on pages 26 and 27.)

TAYlor, P. C.; Silverman, B. W. Block diagrams and splitting criteria for classification trees. Statistics and Computing, vol. 3, pp. 147-161, 1993. (Cited on pages 20, 27, and 92.)

Terashima-Marín, H.; Ross, P.; Farías-ZÁrate, C.; LóPez-Camacho, E.; VALENZUELA-RENDÓN, M. Generalized hyper-heuristics for solving $2 \mathrm{~d}$ regular and irregular packing problems. Annals of Operations Research, vol. 179, no. 1, pp. 369-392, 2010. (Cited on page 54.)

Torgo, L. Functional models for regression tree leaves. In: 14th International Conference on Machine Learning, ICML '97, San Francisco, CA, USA: Morgan Kaufmann Publishers Inc., pp. 385-393, 1997 (ICML '97, ). (Cited on page 39.)

TORGO, L. A comparative study of reliable error estimators for pruning regression trees. In: Iberoamerican Conference on Artificial Intelligence, Springer-Verlag, pp. 1-12, 1998a. (Cited on page 39.)

Torgo, L. Error Estimators for Pruning Regression Trees. In: 10th European Conference on Machine Learning, Springer-Verlag, pp. 125-130, 1998b. (Cited on page 39.)

Tsakonas, A.; Dounias, G. Hierarchical classification trees using type-constrained genetic programming. In: 2002 First International IEEE Symposium on Intelligent Systems, pp. 50-54, 2002. (Cited on pages 64, 66, 69, and 70.)

TURney, P. D. Cost-sensitive classification: Empirical evaluation of a hybrid genetic decision tree induction algorithm. Journal of Artificial Intelligence Research, pp. 369-409, 1995. (Cited on pages 56 and 82.)

UnNikrishnan, K. P.; VenUgopal, K. P. Alopex: a correlation-based learning algorithm for feedforward and recurrent neural networks. Neural Computation, vol. 6, pp. 469-490, 1994. (Cited on page 31.) 
Utgoff, P.; Brodley, C. An incremental method for finding multivariate splits for decision trees. In: 7th International Conference on Machine Learning, pp. 58-65, 1990. (Cited on page 26.)

Utgoff, P. E. Perceptron trees: A case study in hybrid concept representations. Connection Science, vol. 1, no. 4, pp. 377-391, 1989. (Cited on page 28.)

Utgoff, P. E.; Berkman, N. C.; Clouse, J. A. Decision Tree Induction Based on Efficient Tree Restructuring. Machine Learning, vol. 29, no. 1, pp. 5-44, 1997. (Cited on page 43.)

Utgoff, P. E.; Brodley, C. E. Linear machine decision trees. Tech. Rep., University of Massachusetts, Dept of Comp Sci, 1991. (Cited on page 28.)

Utgoff, P. E.; Clouse, J. A. A kolmogorov-smirnoff metric for decision tree induction. Tech report 96-3, University of Massachusetts, 1996. (Cited on pages 19 and 27.)

Uy, N.; O’Neill, M.; Hoai, N.; Mckay, B.; Galván-López, E. Semantic Similarity Based Crossover in GP: The Case for Real-Valued Function Regression. In: Collet, P.; Monmarché, N.; Legrand, P.; Schoenauer, M.; Lutton, E., eds. Lecture Notes in Computer Science, Berlin, Heidelberg: Springer Berlin / Heidelberg SN, pp. 170-181, 2010. (Cited on page 52.)

Varshney, P. K.; Hartmann, C. R. P.; de Faria, J. M. J. Application of Information Theory to Sequential Fault Diagnosis. IEEE Transactions on Computers, vol. 31, no. 2, pp. 164-170, 1982. (Cited on pages 15 and 27.)

VÁzquez-Rodríguez, J. A.; Petrovic, S. A new dispatching rule based genetic algorithm for the multi-objective job shop problem. Journal of Heuristics, vol. 16, no. 6, pp. 771-793, 2010. (Cited on page 53.)

Vella, A.; Corne, D.; MurPhy, C. Hyper-heuristic decision tree induction. World Congress on Nature \& Biologically Inspired Computing, pp. 409-414, 2010. (Cited on pages 4, 54, and 103.)

WANG, D.; JIANG, L. An improved attribute selection measure for decision tree induction. In: 4th International Conference on Fuzzy Systems and Knowledge Discovery, pp. 654-658, 2007. (Cited on pages 17 and 27.)

WANG, Y.; WitTEN, I. H. Induction of model trees for predicting continuous classes. In: Poster papers of the 9th European Conference on Machine Learning, Springer, 1997. (Cited on pages 25,27 , and 74.)

WeISE, T. Global optimization algorithms - theory and application. Second edn.. Self-Published, online available at http://www.it-weise.de/. Access in September, 2009., 2009. Available at http://www. it-weise. de/ (Cited on pages 45 and 50.) 
White, A. P.; LIU, W. Z. Technical Note: Bias in Information-Based Measures in Decision Tree Induction. Machine Learning, vol. 15, no. 3, pp. 321-329, 1994. (Cited on pages 16, 19, and 27.)

WiLcoXon, F. Individual comparisons by ranking methods. Biometrics, vol. 1, pp. 80-83, 1945. (Cited on page 136.)

WILKS, S. S. Mathematical statistics. John Wiley \& Sons Inc., 1962. (Cited on page 15.)

Witten, I. H.; Frank, E. Data mining: Practical machine learning tools and techniques with java implementations. Morgan Kaufmann, 1999. (Cited on pages 37, 101, and 111.)

Wolpert, D. H.; Macready, W. G. No free lunch theorems for optimization. IEEE Transactions on Evolutionary Computation, vol. 1, no. 1, pp. 67-82, 1997. (Cited on page 3.)

WoOdward, J. R. GA or GP? that is not the question. In: IEEE Congress on Evolutionary Computation (CEC 2003), pp. 1056- 1063, 2003. (Cited on page 47.)

Yildiz, C. T.; Alpaydin, E. Omnivariate decision trees. IEEE transactions on Neural Networks, vol. 12, no. 6, pp. 1539-1546, 2001. (Cited on pages 31, 32, and 167.)

Zantema, H.; Bodlaender, H. Finding small equivalent decision trees is hard. International Journal of Foundations of Computer Science, vol. 11, no. 2, pp. 343-354, 2000. (Cited on page 11.)

ZHAO, H. A multi-objective genetic programming approach to developing pareto optimal decision trees. Decision Support Systems, vol. 43, no. 3, pp. 809-826, 2007. (Cited on pages 58, $61,65,66,67,69$, and 70.)

Zhao, Q.; Shirasaka, M. A study on evolutionary design of binary decision trees. In: Angeline, P. J.; Michalewicz, Z.; Schoenauer, M.; Yao, X.; Zalzala, A., eds. IEEE Congress on Evolutionary Computation (CEC 1999), Mayflower Hotel, Washington D.C., USA: IEEE Press, pp. 1988-1993, 1999. (Cited on pages 58, 61, 65, 66, 67, 68, 69, 70, and 83.)

Zhou, X.; Dillon, T. A statistical-heuristic feature selection criterion for decision tree induction. IEEE Transactions on Pattern Analysis and Machine Intelligence, vol. 13, no. 8, pp. 834-841, 1991. (Cited on pages 19 and 27.)

Zorman, M.; Podgorelec, V.; Kokol, P.; Peterson, M.; Sprogar, M.; Ojstersek, M. Finding the right decision tree's induction strategy for a hard real world problem. International Journal of Medical Informatics, vol. 63, no. 1-2, pp. 109 - 121, 2001. (Cited on pages 64, 66, $67,68,83$, and 84.) 